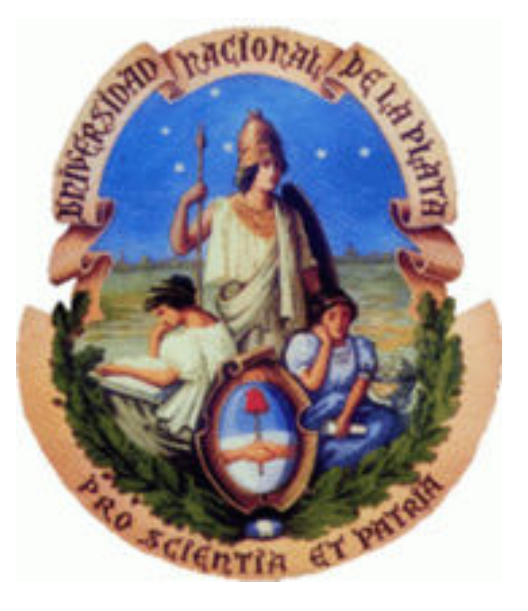

Universidad Nacional de La Plata

Facultad de Ciencias Exactas

Departamento de Física

\title{
Tesis Doctoral \\ Título: Magnetismo cuántico y fases aisladoras en sistemas de electrones correlacionados en bajas dimensiones
}

\author{
Lic. Héctor Diego Rosales \\ Director: Dr. Gerardo L. Rossini
}

Septiembre de 2010 
Rosales, Héctor Diego

Magnetismo cuántico y fases aisladoras en sistemas de electrones correlacionados en bajas dimensiones. - 1a ed. - La Plata : Universidad Nacional de La Plata, 2011.

E-Book.

ISBN 978-950-34-0797-4

1. Electrones. 2. Tesis de doctorado. I. Título CDD 539.72112

Fecha de catalogación: 11/11/2011 
A Oriana por estar siempre a mi lado y por su apoyo incondicional. 


\section{Agradecimientos}

Agradezco al CONICET por el financiamiento de mis carrera de doctorado.

A mi director Dr. Gerardo Luis Rossini por aceptarme como tesista y guiarme en el camino de la investigación científica.

Al Dr. Daniel Carlos Cabra por su gran apoyo, su generosidad y discusiones científicas que me han sido de gran utilidad para llevar adelante este Doctorado.

A Nicolas y Guillermo quienes me han brindado una grata companía y con los cuales he compartido amenas discusiones científicas.

A mis compañeros de oficina Carlos y Juan Cruz por los buenos momentos compartidos.

A mi papá y a mi mamá por brinadarme un gran apoyo a lo largo de toda mi carrera. 


\section{Resumen}

El trabajo llevado a cabo en esta tesis se encuentra dentro del marco de la física de la materia condensada y, más precisamente, se centra en los sistemas electrónicos fuertemente correlacionados cuya dimensión espacial es menor que tres.

Los sistemas fuertemente correlacionados son sistemas electrónicos en los cuales la interacción coulombiana no puede ser tratada perturbativamente en comparación con los otros grados de libertad del sistema. Como consecuencia, estos sistemas son verdaderos "sistemas de muchos cuerpos" y en la mayoría de los casos, es necesario utilizar tanto técnicas numéricas como analíticas para poder hacer frente a su estudio. Más aún, las correlaciones inducidas por las interacciones son drásticamente amplificadas a medida que la dimensión del sistema es reducida, lo cual hace que el estudio de los sistemas en una y dos dimensiones sea de gran relevancia.

En muchos sistemas electrónicos, las interacciones electrónicas pueden producir efectos drásticos llevando a un completo colapso del estado metálico, incluso cuando la banda de conducción está parcialmente ocupada. El caso más simple es la conocida fase de aislador de Mott [1] en el cual hay únicamente un electrón por celda unidad cristalina, y por lo tanto se esperaría una banda de conducción metálica a medio llenado.

Durante las últimas dos o tres décadas, experimentos realizados con distintos tipos de materiales han evidenciado que las correlaciones fuertes son un ingrediente central para la comprensión de sus propiedades físicas. Entre estos materiales están los superconductores de alta temperatura crítica, materiales que presentan magnetorresistencia colosal (CMR), efecto Hall cuántico fraccionario (FQHE), nanotubos de carbono, una variedad de metales orgánicos, conductores y dieléctricos, entre otros. En particular, las propiedades físicas de sistemas en bajas dimensiones han impulsado innumerable investigaciones en gran parte de siglo pasado, y se han convertido en uno de los primeros temas de interés en investigación de materia condensada.

Dentro de la problemática de electrones fuertemente correlacionados, aquellos sistemas en los cuales los grados de libertad relevantes son los espines de los electrones constituyen el magnetismo cuántico. Estos sistemas se modelan usualmente utilizando hamiltonianos de Heisenberg con interacciones de intercambio $(J \sim 10 \mathrm{meV})$ dominantes entre espines localizados que determinan una red periódica cuya geometría es decisiva para sus propiedades físicas. Dos ingredientes básicos pueden identificarse como cruciales en el comportamiento de estos sistemas: las interacciones fuertes y la frustración. La combinación de estos dos ingredientes conduce a elevadas fluctuaciones cuánticas que pueden dar lugar a una variedad muy rica de fases magnéticamente ordenadas que se observan a bajas temperaturas en diferentes materiales, incluyendo desde las ondas de espín hasta la exótica fase de vidrio de espín. En particular en los sistemas de espines de baja dimensionalidad las fluctuaciones cuánticas tienen efectos más relevantes que en tres dimensiones: la competencia entre el orden (para minimizar la energía) y el desorden (por fluctuaciones cuánticas) es delicada y generalmente no se establece a temperatura cero un orden de largo alcance, esto significa que espines ubicados en sitios de la red cuya separación es varias veces el espaciamiento 
de la red, no presentan correlación entre sí. En particular, existen materiales en los cuales la red de espines en interacción resulta una estructura unidimensional (cadenas de espines), cuasi-unidimensional (escaleras de espines) o bidimensional (planos de espines), esencialmente desacoplada del resto del sistema. El caso paradigmático de este fenómeno es la estructura de planos de cobre y oxígeno en los superconductores de alta temperatura, que forman una red cuadrada con interacciones antiferromagnéticas entre primeros vecinos.

La aparición de fases cuánticas exóticas en sistemas descritos por antiferromagnetos con frustración (por ejemplo, sistemas que presentan interacciones competitivas antiferromagnéticas) es actualmente objeto de intensa investigación.

La presente tesis está enfocada al estudio de las propiedades del estado fundamental de sistemas tanto de electrones como de espines en bajas dimensiones. En la primera parte nos centramos en la problemática de los sistemas de electrones y de espines en una dimensión espacial, y analizamos el rol que juegan los fonones y la frustración magnética. En cuanto a los fonones, son considerados únicamente en el límite adiabático en el que sus frecuencias son suficientemente bajas como para descartar la dinámica de éstos. Consecuentemente, ellos son tratados como variables clásicas relacionadas con pequeños desplazamientos de los sitios de la red. Trabajos previos tanto en sistemas de electrones como de espines, han mostrado que la inclusión de los fonones tiene como consecuencias la aparición de fases exóticas.

En la segunda parte analizamos el rol de la frustración en las propiedades magnéticas de los sistemas magnéticos bidimensionales haciendo hincapié en la búsqueda de fases desordenadas conocidas como líquidos de espines.

La tesis está organizada de la siguiente manera. En el capítulo 1 a modo de introducción, sentamos los fundamentos teóricos de los sistemas cuánticos estudiados. Primero introducimos modelos para los sistemas fuertemente correlacionados. En particular, presentamos el modelo de Hubbard y algunos de sus límites como el hamiltoniano de Heisenberg. Además, analizamos las propiedades generales de tales modelos. Discutimos brevemente algunos resultados experimentales de sistemas fuertemente correlacionados en bajas dimensiones que muestran propiedades muy interesantes a bajas temperaturas. El capítulo 2 contiene una descripción de los métodos analíticos y numéricos que hemos empleados en los estudios realizados, resaltando el alcance de éstos y sus limitaciones. Los capítulos 3 y 4 están dedicados al estudio de sistemas correlacionados en una dimensión. En el capítulo 3 estudiamos las propiedades electrónicas del estado fundamental del modelo de Hubbard a llenado 1/4, en presencia de fonones adiabáticos. El capítulo 4 estudiamos las propiedades del estado fundamental del modelo de Heisenberg antiferromagnético en presencia de frustración magnética y acoplamiento espín-fonón.

El capítulo 5 está dedicado al estudio de los sistemas magnéticos frustrados en dos dimensiones. Primero comentamos las motivaciones actuales para estudiar estos sistemas. Posteriormente estudiamos las propiedades magnéticas del modelo de Heisenberg frustrado sobre la red de Kagomé y la red hexagonal, en función de los distintos acoplamientos y la magnitud del espín $S$. En el capítulo 6 presentamos las conclusiones generales y las perspectivas sobre futuros trabajos relacionados con los temas aquí tratados. Finalmente, presentamos las referencias usadas y los apéndices. 


\section{Índice general}

$\begin{array}{lc}\text { Índice general } & 6\end{array}$

1. Fundamentos teóricos y resultados previos $\quad 9$

1.1. Interacción electrónica en sólidos . . . . . . . . . . . . . . . . . 10

1.2. Modelo de Hubbard . . . . . . . . . . . . . . . . . . . . . 12

1.2.1. Simetrías del modelo de Hubbard . . . . . . . . . . . . . . . . . 14

1.2.2. Límites particulares . . . . . . . . . . . . . . . . 16

1.2.3. Líquidos de Fermi y Luttinger . . . . . . . . . . . . . . . . . . . 18

1.3. Modelo de Heisenberg . . . . . . . . . . . . . . . . . . . . . 22

1.3.1. Origen microscópico de las interacciones magnéticas . . . . . . . . . 25

1.3.2. Orden de largo alcance: teorema de Mermin-Wagner . . . . . . . . . . 26

1.3.3. Sistemas desordenados y líquidos de espines . . . . . . . . . . . . . 28

1.3.4. Sistemas frustrados . . . . . . . . . . . . . . . . . . 31

1.4. Acoplamiento electrón-fonón . . . . . . . . . . . . . . . . . 36

2. Metódos Analíticos y Numéricos $\quad 39$

2.1. Bosonización Abeliana, $d=1 \ldots \ldots \ldots \ldots$. . . . . . . . . 39

2.1.1. Fermiones libres . . . . . . . . . . . . . . . . . . . 40

2.1.2. Fermiones interactuantes . . . . . . . . . . . . . . . . . 42

2.1.3. Reglas de bosonización . . . . . . . . . . . . . . . . . . . . 44

2.1.4. Bosonización del Modelo de Hubbard y Heisenberg . . . . . . . . . . 45

2.2. Bosonización de los operadores de espín, $d>1$. . . . . . . . . . . . . 51

2.2.1. Bosones de Holstein-Primakoff y ondas de espín . . . . . . . . . . . . 51

2.2.2. Método de Bosones de Schwinger . . . . . . . . . . . . . . . . 55

2.3. Métodos Numéricos . . . . . . . . . . . . . . . . . . . . . 59

2.3.1. Algoritmo de Lanczos . . . . . . . . . . . . . . . . . . . . . . . . . . . 60

2.3.2. Método de Renormalización de la Matriz Densidad: DMRG . . . . . . 62

I Sistemas unidimensionales acoplados con la red $\quad 65$

3. Modelo de Hubbard acoplado con la red $\quad \mathbf{6 7}$

3.1. Orden de carga en conductores moleculares y deformaciones de la red subyacente. 67 
3.2. Hamiltoniano de Hubbard extendido . . . . . . . . . . . . . . . . 70

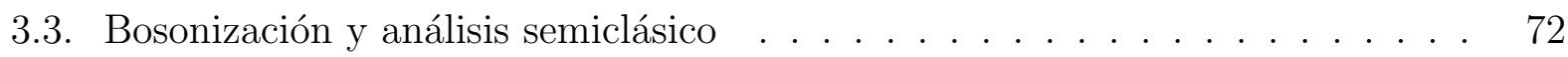

3.4. Resultados . . . . . . . . . . . . . . . . . . . 77

3.5. Conclusiones . . . . . . . . . . . . . . . . . . . 78

4. Modelo de Heisenberg acoplado con la red $\quad 81$

4.1. Introducción . . . . . . . . . . . . . . . . . . 81

4.2. Transiciones de fase cuánticas en cadenas trimerizadas. . . . . . . . . . . . 83

4.2.1. Frustración vs modulación . . . . . . . . . . . . . . . 86

4.2.2. Límite de frustración fuerte . . . . . . . . . . . . . . . . . . 88

4.2.3. Límite de modulación débil . . . . . . . . . . . . . . . . . . 93

4.2.4. Análisis numérico . . . . . . . . . . . . . . . . . . . . . . . . . . . . . . . . . . . . . . . . 95

4.2.5. Resultados y discusión . . . . . . . . . . . . . . . . . . . 99

4.3. Fases tipo espín-Peierls en cadenas de espines a $M=1 / 3 \ldots \ldots$. . . . . . 100

4.3.1. Introducción . . . . . . . . . . . . . . . . . . . . . 100

4.3.2. Descripción del modelo y bosonización . . . . . . . . . . . . . . 102

4.3.3. Análisis semiclásico de la teoría efectiva . . . . . . . . . . . . . . 106

4.3.4. Análisis numérico . . . . . . . . . . . . . . . . . . 109

4.3.5. Conclusiones ........................ 111

II Sistemas Magnéticos en 2D 113

5. Modelo de Heisenberg $\quad 115$

5.1. Modelo de Heisenberg en la red de Kagomé. . . . . . . . . . . . . . . . . . . 116

5.1.1. Introducción . . . . . . . . . . . . . . . . . . . 116

5.1.2. Métodos de bosones de Schwinger . . . . . . . . . . . . . . . 118

5.1.3. Resultados . . . . . . . . . . . . . . . . . 121

5.2. Modelo de Heisenberg en la red hexagonal. . . . . . . . . . . . . . . . . . 122

5.2.1. Aspectos clásicos y semi-clásicos . . . . . . . . . . . . . . . . . 123

5.2.2. Método de bosones de Schwinger . . . . . . . . . . . . . . 127

5.2.3. Resultados de la teoría de campo medio. . . . . . . . . . . . . . . 129

5.2.4. Discusión y Conclusiones . . . . . . . . . . . . . . . . 132

6. Conclusiones generales y perspectivas 135

6.0.5. Técnicas empleadas. . . . . . . . . . . . . . . . . . . . . . . . . . . . . . . . 135

6.0.6. Modelos estudiados. . . . . . . . . . . . . . . . . . . 135

6.0.7. Trabajos en ejecución y perspectivas futuras. . . . . . . . . . 136

$\begin{array}{ll}\text { Bibliografía } & 137\end{array}$ 


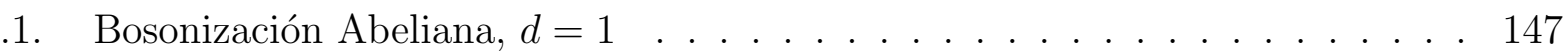

.1.1. Teoría efectiva de baja energía del modelo de Hubbard . . . . . . . . 147

.1.2. Teorías conformes . . . . . . . . . . . . . . 150

.1.3. Reglas de bosonización . . . . . . . . . . . . . . . . . . . 152

.1.4. Bosonización del acoplamiento electrón-fonón, espín-fonón. . . . . . . 154

.2. Diagonalización paraunitaria. . . . . . . . . . . . . . 157 


\section{Capítulo 1}

\section{Fundamentos teóricos y resultados previos}

En este capítulo y el siguiente se establecen los fundamentos teóricos para estudiar diversos sistemas fuertemente correlacionados que serán utilizados en los próximos capítulos como herramientas para el análisis de los modelos propuestos.

Por muchos años, la teoría de perturbaciones diagramática ha sido la mayor herramienta teórica para tratar las interacciones en metales, semiconductores, magnetos itinerantes, y superconductores. Ésta consiste en esencia, en un desarrollo en el límite de acoplamiento débil considerando estados de cuasi-partículas. Sin embargo, una gran cantidad de descubrimientos experimentales durante las últimas décadas, incluyendo fermiones pesados, efecto Hall cuántico fraccionario, superconductividad de alta temperatura, y cadenas de espines cuánticas, no son (en la mayoría de lo casos) realmente accesibles desde el límite de acoplamiento débil. Esto ha impulsado a desarrollar como alternativa, herramientas no perturbativas para poder manejar las fuertes interacciones electrón-electrón.

En particular, nos vamos a concentrar en dos paradigmas básicos de sistemas cuánticos fuertemente interactuantes: el modelo de Hubbard y el modelo de Heisenberg. Estos modelos son vehículos para comprender conceptos fundamentales, tales como hamiltonianos efectivos de modelos más complicados, estados fundamentales variacionales, fenómenos tales como ruptura espontánea de simetría y desorden cuántico. En adición, ellos son usados como laboratorio para varios esquemas de aproximaciones no perturbativos que han encontrado aplicaciones en diversas áreas de la física teórica.

En el marco del modelo de Hubbard, exponemos los líquidos electrónicos y discutimos el efecto de las correlaciones como función de la dimensión espacial del sistema. Revisaremos el modelo de líquido de Fermi que describe sistemas bidimensionales y tridimensionales y por otro lado, presentaremos el líquido de Luttinger para sistemas unidimensionales. Como veremos, en una dimensión los efectos de las correlaciones son extremadamente importantes a tal punto que las excitaciones fundamentales se vuelven colectivas, y por lo tanto no pueden ser descritas en término de excitaciones individuales como ocurre en un líquido de Fermi. Posteriormente discutiremos el origen de la interacciones magnéticas y presentaremos el mo- 


\section{Interacción electrónica en sólidos}

delo de Heisenberg. Expondremos brevemente la posibilidad de orden magnético, la ruptura de simetría, los sistemas que presentan estados magnéticamente desordenados conocidos como líquidos de espines y el papel que juega la temperatura en dichos sistemas presentando el teorema de Mermin y Wagner. En la parte final introduciremos el concepto de frustración magnética que será mencionado ampliamente en el resto de la tesis. En particular, examinaremos los dos posibles orígenes de frustración: (a) debido la geometría de la red, y (b) a las interacciones competitivas.

\subsection{Interacción electrónica en sólidos}

En esta sección revisaremos algunos de los modelos más utilizados para describir sistemas en materia condensada. Comenzaremos con el modelo de electrones libres en una red, luego incluiremos la interacción coulombiana y finalmente obtendremos el hamiltoniano de Hubbard de una banda que será ampliamente discutido. Presentaremos brevemente las hipótesis que son usadas en este modelo.

Comencemos con el caso en que la interacción entre los electrones no es tenida en cuenta. Supongamos un sistema de $N_{e}$ electrones en una red periódica de volumen $V=L_{x} \cdot L_{y} \cdot L_{z}$ (donde $L_{x}, L_{y}, L_{z}$ son las longitudes del sistema en las tres dimensiones espaciales) que representa la estructura de un cristal. La ecuación de Schrödinger para un electrón en un potencial periódico $\mathcal{V}_{i o n}(\mathbf{r})$ está dada por:

$$
\left[-\frac{\hbar^{2}}{2 m} \nabla^{2}+\mathcal{V}_{i o n}(\mathbf{r})\right] \varphi_{\alpha, \mathbf{k}}(\mathbf{r})=E_{\alpha}(\mathbf{k}) \varphi_{\alpha, \mathbf{k}}(\mathbf{r})
$$

Las soluciones de la ecuación anterior corresponden a las funciones de Bloch $\varphi_{\alpha, \mathbf{k}}[2,3,4]$, donde $\alpha$ es el índice de banda y $\mathbf{k}$ es el vector de onda definido en la primera zona de Brillouin (PZB). La superficie de Fermi está definida por $E_{\alpha}(\mathbf{k})=E_{F}$, donde $E_{F}$ es la energía de Fermi. Las funciones de Bloch están definidas como el producto de una onda plana describiendo un electrón libre veces una función $u_{\alpha, \mathbf{k}}(\mathbf{r})$ que tiene la misma simetría de traslación que la red. Así, la función de Bloch queda

$$
\varphi_{\alpha, \mathbf{k}}(\mathbf{r})=u_{\alpha, \mathbf{k}}(\mathbf{r}) \cdot e^{i \mathbf{k} \cdot \mathbf{r}} .
$$

Teniendo en cuenta todas las bandas de energía, el operador de campo de un electrón con espín $\sigma$ en el sitio $\mathbf{r}$ puede ser expresado como [4]

$$
\psi_{\sigma}^{\dagger}(\mathbf{r})=\sum_{\alpha, \mathbf{k}} \varphi_{\alpha, \mathbf{k}}^{*}(\mathbf{r}) c_{\alpha, \mathbf{k}, \sigma}^{\dagger}
$$

De esta manera, podemos escribir el hamiltoniano de la ecuación (1.1) en términos de operadores de creación y aniquilación como:

$$
\mathcal{H}_{\text {libre }}=\sum_{\alpha, \mathbf{k} \sigma} E_{\alpha}(\mathbf{k}) c_{\alpha, \mathbf{k}, \sigma}^{\dagger} c_{\alpha, \mathbf{k}, \sigma} .
$$


En sistemas en donde la densidad de electrones es muy baja o bien la repulsión coulombiana es fuertemente apantallada, el hamiltoniano definido en la ecuación (1.4) es una muy buena aproximación para describir tanto propiedades electrónicas como magnéticas de muchos sistemas [5].

Consideremos ahora la interacción entre los electrones $\mathcal{V}^{\text {int }}\left(\mathbf{r}, \mathbf{r}^{\prime}\right)$. Además de la parte libre (1.4), el hamiltoniano contendrá el término de interacción dado por

$$
\mathcal{H}_{\text {int }}=\frac{1}{2} \sum_{i \neq j} \mathcal{V}^{\text {int }}\left(\mathbf{r}_{i}, \mathbf{r}_{j}\right)
$$

$\mathrm{Al}$ incluir este término, surge el problema de que la interacción es de largo alcance y entonces cada partícula del sistema interactúa con el resto. Así, el hamiltoniano no puede ser más tratado como una suma de términos independientes y es necesario realizar aproximaciones [4]. Muchos de los efectos de la interacción de Coulomb pueden ser incorporados dentro del término cinético modificando el potencial iónico,

$$
\mathcal{H}_{\text {libre }} \rightarrow \tilde{\mathcal{H}}_{\text {libre }}=\sum_{i=1}^{N_{e}}\left[-\frac{\hbar^{2}}{2 m} \nabla_{i}^{2}+\mathcal{V}_{\text {ion }}\left(\mathbf{r}_{i}\right)+\mathcal{V}^{\text {eff }}\left[\mathbf{r}_{i}, \rho\right]\right]
$$

donde $\mathcal{V}^{\text {eff }}\left[\mathbf{r}_{i}, \rho\right]$ es una funcional de la densidad electrónica del estado fundamental y que puede ser calculada mediante métodos aproximados [4].

La interacción residual es

$$
\tilde{\mathcal{V}}^{\text {int }}\left(\mathbf{r}_{i}, \mathbf{r}_{j}\right)=\mathcal{V}^{\text {int }}\left(\mathbf{r}_{i}, \mathbf{r}_{j}\right)-\left[\mathcal{V}^{\text {eff }}\left[\mathbf{r}_{i}, \rho\right]+\mathcal{V}^{e f f}\left[\mathbf{r}_{j}, \rho\right]\right] / N_{e}
$$

se denomina término de screening (o término de apantallamiento). El lector interesado, puede consultar más detalles a cerca del término de apantallamiento (ver por ejemplo Auerbach [4] y referencias). A partir de ahora y posteriormente cuando presentemos el modelo de Hubbard, consideraremos electrones interactuando a través de la interacción apantallada. Utilizando los operadores de campo de los electrones, el hamiltoniano de interacción que incluye el potencial apantallado $\tilde{\mathcal{V}}^{\text {int }}\left(\mathbf{r}, \mathbf{r}^{\prime}\right)$ queda,

$$
\mathcal{H}_{i n t}=\int d \mathbf{r} d \mathbf{r}^{\prime} \psi_{\sigma}^{\dagger}(\mathbf{r}) \psi_{\sigma^{\prime}}^{\dagger}\left(\mathbf{r}^{\prime}\right) \tilde{\mathcal{V}}^{i n t}\left(\mathbf{r}, \mathbf{r}^{\prime}\right) \psi_{\sigma^{\prime}}\left(\mathbf{r}^{\prime}\right) \psi_{\sigma}(\mathbf{r})
$$

Uno de los enfoques más conocidos en física del estado sólido es el que se conoce como tightbinding. En éste, las funciones de onda de los electrones se encuentran bien localizadas en cada sitio de la red, y el solapamiento de funciones de onda localizadas en sitios vecinos es muy débil; así, en vez de trabajar con las funciones de Bloch que son deslocalizadas en el espacio real, uno define un nuevo conjunto de funciones denominadas funciones de Wannier [6], que son localizadas alrededor de los sitios atómicos $j(j=1, \ldots, N)$. Estas están definidas como transformadas de Fourier de las funciones de Bloch,

$$
\phi_{\alpha}\left(\mathbf{r}-\mathbf{r}_{j}\right)=\frac{1}{\sqrt{N}} \sum_{\mathbf{k} \in \mathrm{PZB}} e^{-i \mathbf{k} \cdot \mathbf{r}_{j}} \varphi_{\mathbf{k}_{j}, \alpha}(\mathbf{r}) .
$$


La misma transformada define los operadores de Wannier $\left(c_{\alpha, j, \sigma}^{\dagger} \mathrm{y} c_{\alpha, j, \sigma}\right)$, con los cuales el operador de campo de un electrón está dado por

$$
\psi_{\sigma}^{\dagger}(\mathbf{r})=\sum_{\alpha, j} \phi_{\mathbf{r}_{j}, \alpha}^{*}(\mathbf{r}) c_{\alpha, j, \sigma}^{\dagger},
$$

donde $\alpha$ es el índice de banda.

Cuando las escalas de energía involucradas en los procesos que tienen lugar en el sistema (como la temperatura, frecuencias externas, etc.) son pequeñas frente a la separación entre bandas (o más precisamente, con la separación entre la banda de conducción y la siguiente), únicamente la última resulta relevante para la descripción del sistema dado que no hay modo de producir excitaciones que pueblen las bandas superiores. En este caso, podemos escribir

$$
\psi_{\sigma}^{\dagger}(\mathbf{r})=\sum_{j} \phi_{\mathbf{r}_{j}}^{*}(\mathbf{r}) c_{j, \sigma}^{\dagger}
$$

Esta aproximación debe ser mejorada en los sistemas llamados multi-bandas, como compuestos de tierras raras (metales con electrones- $f$ ), en donde los parámetros de interacción son del mismo orden de magnitud que la separación entre bandas.

Bajo estas suposiciones, el hamiltoniano completo (término cinético y de interacción) en término de los operadores de Wannier queda,

$$
\begin{aligned}
\mathcal{H}_{\text {Wannier }} & =-\sum_{i, j, \sigma} t_{i j}\left(c_{i, \sigma}^{\dagger} c_{j, \sigma}+\text { h.c. }\right)+\sum_{i, j, k, l, \sigma, \sigma^{\prime}} U_{i j k l}\left(c_{i, \sigma}^{\dagger} c_{j, \sigma^{\prime}}^{\dagger} c_{k, \sigma^{\prime}} c_{l, \sigma}+\text { h.c. }\right) \\
t_{i j} & =\frac{1}{N} \sum_{\mathbf{k} \in P Z B} e^{-i\left(\mathbf{r}_{j}-\mathbf{r}_{i}\right) \cdot \mathbf{k}} E(\mathbf{k}) \\
U_{i j k l} & =\frac{1}{2} \int d \mathbf{r} d \mathbf{r}^{\prime} \phi_{\mathbf{r}_{i}}^{*}(\mathbf{r}) \phi_{\mathbf{r}_{j}}^{*}\left(\mathbf{r}^{\prime}\right) \tilde{\mathcal{V}}^{i n t}\left(\mathbf{r}, \mathbf{r}^{\prime}\right) \phi_{\mathbf{r}_{k}}\left(\mathbf{r}^{\prime}\right) \phi_{\mathbf{r}_{l}}(\mathbf{r}),
\end{aligned}
$$

donde la sigla PZB indica la primera zona de Brillouin [3], los parámetros $t_{i j}>0$ se denominan elementos de la matriz de hopping (o constante de hopping) y $U_{i j k l}$ son los parámetros de interacción entre los electrones.

Puesto que el potencial de interacción apantallado, $\tilde{\mathcal{V}}^{i n t}\left(\mathbf{r}, \mathbf{r}^{\prime}\right)$ es de corto alcance, éste decrece rápidamente cuando la separación $\left|\mathbf{r}-\mathbf{r}^{\prime}\right|$ aumenta. Así, la aproximación más simple consiste en tomar $U_{i j k l}=0$ que corresponde a electrones libres; como corrección se considera la interacción coulombiana en el sitio, $U_{i i i i} \equiv U$ y finalmente se puede tener en cuenta parámetros de hopping e interacción coulombiana a siguientes vecinos.

\subsection{Modelo de Hubbard}

Esta sección es dedicada a uno de los modelos más simples para describir sistemas de muchos cuerpos y que no puede ser reducido a una teoría de partícula independiente: el 
modelo de Hubbard [7, 8]. Introduciendo los operadores números $n_{i, \sigma}=c_{i, \sigma}^{\dagger} c_{i, \sigma}, \sigma=\uparrow, \downarrow \mathrm{y}$ reemplazando $U_{i i i i} \equiv U$, el hamiltoniano de Hubbard es

$$
\mathcal{H}_{\text {Hubbard }}=-\sum_{<i, j>, \sigma} t_{i j}\left(c_{i, \sigma}^{\dagger} c_{j, \sigma}+\text { h.c. }\right)+U \sum_{i} n_{i, \uparrow} n_{i, \downarrow}-\mu \sum_{i, \sigma=\uparrow, \downarrow} n_{i, \sigma}
$$

donde hemos introducido el multiplicador de Lagrange $\mu$ (potencial químico) que regula el llenado del sistema (el número de electrones), y el símbolo $\langle i, j\rangle$ indica que que la suma es a primeros vecinos. Una de las motivaciones para estudiar el modelo de Hubbard, es que éste resulta ser la generalización más simple, más allá de la descripción de la teoría de bandas en sólidos, y sin embargo, captura las características físicas más importantes de muchos sistemas [8].

A pesar de su aparente simplicidad, no existe ningún tratamiento que permita estudiar el modelo de manera general para cualquier rango de los parámetros y en cualquier dimensión espacial. Sin embargo, hay dos casos particulares en los cuales muchas de sus propiedades son calculables de manera exacta, ellos son los casos extremos del número de coordinación de la red: dos e infinito. En el primer caso, que corresponde a una red o cadena unidimensional, el modelo de Hubbard tiene una característica particular: es integrable, es decir, se puede resolver de manera exacta. Este problema fue resuelto por Lieb y $\mathrm{Wu}$ [9] utilizando una extensión del ansatz de Bethe [10] para fermiones. Así, la energía del estado fundamental a medio llenado (un electrón por sitio) esta dada por

$$
\frac{E_{0}}{N_{e}}=-|t| \int_{0}^{\infty} \frac{d x J_{0}(x) J_{1}(x)}{x[1+\exp (x U /|t|)]},
$$

donde $J_{n}(x)$ es la función de Bessel. El estado fundamental en el límite $U<<t$ (límite de banda) es

$$
\frac{E_{0}}{N_{e}}=-|t| \frac{|t|}{\pi}+\frac{U}{4}-0.0017 \frac{U^{2}}{|t|}
$$

mientras que en el límite atómico $U>>t$, es

$$
\frac{E_{0}}{N_{e}}=-4|t|^{2} \log 2
$$

El estado fundamental a medio llenado es un aislador para todos los valores de $U / t$. Esto se debe a que el potencial químico requerido para agregar un electrón $\mu_{+}$es más grande que el potencial para remover uno $\mu_{-}$. La relación $\mu_{+}>\mu_{-}$es típica para un aislador con un gap de energía entre las bandas llena y vacía. La función de onda exacta del estado fundamental está dada por el ansatz de Bethe y posee una estructura complicada que puede ser simplificada en el límite $U \rightarrow \infty$ cuando los estados de doble ocupación en cada sitio pueden ser excluidos.

En el límite de coordinación infinita, dimensión espacial $d \rightarrow \infty$, las soluciones no-triviales para el modelos de Hubbard fueron encontradas por Metzner y Vollhardt [11]. En este límite la autoenergía se vuelve local [12]:

$$
\Sigma_{i j}(w)=\Sigma(w) \delta_{i j}
$$




\section{Modelo de Hubbard}

Esto hace posible construir una teoría de campo medio no trivial (que es exacta en el límite $d \rightarrow \infty)$ que presenta los principales resultados esperados en $d=3$ : la transición metalaislador, el orden antiferromagnético en el caso de medio llenado, el comportamiento tipo líquido de Fermi (ver sección 1.2.3) en la fase metálica. Existen varios trabajos dedicados al límite $d \rightarrow \infty$ del modelo de Hubbard $[13,14,15]$.

En casos más generales, el estado fundamental y las excitaciones, tanto del modelo de Hubbard como de sus variantes (como por ejemplo, la inclusión de campo magnético, interacción con fonones, desorden,...,etc.), han sido estudiados de varias maneras. En una dimensión espacial $(d=1)$, existen varios métodos analíticos entre los cuales podemos citar: bosonización abeliana (el cual desarrollaremos posteriormente), métodos de teoría de campos, modelos de Tomonaga-Luttinger y grupo de renormalización perturbativo. Del lado de métodos numéricos podemos citar el algoritmo de Lanczos, el método de diagonalización de la matriz densidad (DMRG) y Montecarlo cuántico (los primeros dos, serán desarrollados en el capítulo siguiente). En el caso de dos dimensiones $(d=2)$, no existen métodos analíticos similares a los de $d=1$, sin embargo los progresos se han hecho en varios regímenes del espacio de parámetros usando combinación de teoremas, aproximaciones controladas, y resultados numéricos (como diagonalización exacta y Montecarlo cuántico) extrapolados a redes grandes.

\subsubsection{Simetrías del modelo de Hubbard unidimensional}

El hamiltoniano de Hubbard posee muchas simetrías. Algunas de ellas, como la simetría de traslación que surge al considerar hoppings homogéneos $\left(t_{i j}=t\right)$ o la simetría frente a la inversión del indice de espín $\sigma \rightarrow-\sigma$, son comunes. Sin embargo, hay otras que son menos usuales.

En esta sección vamos a mencionar algunas de las simetrías más importantes. Para más detalles sobre el tema, se puede consultar una gran cantidad de bibliografía $([8,16])$.

- La transformación partícula-hueco

$$
c_{i, \sigma} \rightarrow(-1)^{i} c_{i, \sigma}^{\dagger}
$$

deja inalterados los términos cinéticos y de interacción, mientras que cambia $\mu \rightarrow-\mu$. De esta manera, es equivalente estudiar el sistema con dopaje de electrones o huecos.

- La transformación de escala o calibre, más comúnmente llamada transformación de gauge,

$$
\tilde{c}_{i, \sigma} \rightarrow e^{i \theta} c_{i, \sigma}
$$

corresponde a una transformación $U(1)$ en los operadores fermiónicos. Esta simetría es una consecuencia de la conservación de la carga (o el número de electrones). 
- Una simetría muy interesante que surge a medio llenado, es la simetría partícula-hueco sobre una de las especies fermiónicas, es decir

$$
\begin{array}{r}
c_{i, \downarrow} \rightarrow(-1)^{i} c_{i, \downarrow}^{\dagger} \\
c_{i, \uparrow} \rightarrow c_{i, \uparrow}
\end{array}
$$

Esta transformación deja el término cinético sin cambios pero cambia $U \rightarrow-U$ obteniendo un término atractivo. En este caso para grandes valores de $U$, se espera que pares de electrones con espines opuestos, formen singletes en cada sitio. De esta manera, se podría esperar que en dimensión $d=3$, estos estados de carácter bosónico (al estar formados por dos fermiones) podrían condensar llevando al sistema a una fase supercondutora.

- El sector de espín tiene invariancia $S U(2)$. Para considerar esta simetría definimos el operador de espín en el sitio $i$,

$$
S_{i}^{(a)}=\frac{1}{2} \sum_{\sigma, \sigma^{\prime}} c_{i, \sigma}^{\dagger}\left(\tau^{(a)}\right)_{\sigma \sigma^{\prime}} c_{i, \sigma^{\prime}}
$$

con $a=x, y, z$ y $\tau^{(a)}$ las matrices de Pauli,

$$
\tau_{x}=\left(\begin{array}{ll}
0 & 1 \\
1 & 0
\end{array}\right), \quad \tau_{y}=\left(\begin{array}{rr}
0 & -i \\
i & 0
\end{array}\right), \quad \tau_{z}=\left(\begin{array}{rr}
1 & 0 \\
0 & -1
\end{array}\right)
$$

El espín total del sistema es igual a

$$
S_{\text {tot }}^{(a)}=\sum_{i} S_{i}^{(a)}
$$

Los operadores $S_{t o t}^{(a)}$ conmutan con los términos cinéticos y de interacción del hamiltoniano 1.15, lo que significa que el hamiltoniano de Hubbard posee una invarianza frente a rotaciones globales en el subespacio de espín. Los números cuánticos son las proyecciones $z$ de $S_{t o t}^{(z)}$ y el valor del espín total $S_{t o t}\left(S_{t o t}+1\right)$ con el operador de espín

$$
S_{t o t}^{2}=S_{t o t}^{(x)^{2}}+S_{t o t}^{(y)^{2}}+S_{t o t}^{(z)^{2}}
$$

Para un dado número de electrones $N_{e} \mathrm{y}$ sitios $N$, uno puede encontrar un valor máximo del espín total

$$
S_{\text {max }}=\left\{\begin{array}{cc}
N_{e} / 2 & 0 \leq N_{e} \leq N \\
N-N_{e} / 2 & 0 \leq N \leq N_{e} \leq 2 N
\end{array}\right.
$$

siendo los posibles valores de $S_{t o t}$

$$
S_{\text {tot }}=0,1, \ldots, S_{\max } \quad\left(S_{t o t}=1 / 2,3 / 2, \ldots, S_{\max }\right)
$$

para un número par (impar) de electrones. 
- Pseudo-espín.

El modelo de Hubbard posee una simetría adicional llamada de Pseudo-espín. El vector de pseudo-espín se define de manera similar al de la ecuación (1.21):

$$
T_{i}=\frac{1}{2} \Psi_{i, \sigma^{\prime}}^{\dagger} \tau_{\sigma, \sigma^{\prime}} \Psi_{i, \sigma^{\prime}}
$$

con $\tau=\left(\tau^{(x)}, \tau^{(y)}, \tau^{(z)}\right)$ y el espinor de dos componentes $\Psi_{i, \sigma}$ dado por

$$
\Psi_{i}=\left(\begin{array}{c}
c_{i, \uparrow} \\
(-1)^{i} c_{i, \downarrow}^{\dagger}
\end{array}\right)
$$

Estos operadores forman un álgebra de $\mathrm{SU}(2)$ y siguen las usuales relaciones de conmutación. A partir de estos se puede definir el operador de pseudo-espín total

$$
\begin{aligned}
T_{t o t}^{(a)} & =\sum_{i} T_{i}^{(a)} \\
T_{t o t}^{2} & =T_{t o t}^{(x)^{2}}+T_{t o t}^{(y)^{2}}+T_{t o t}^{(z)^{2}}
\end{aligned}
$$

Las siguientes relaciones de conmutación,

$$
\left[\mathcal{H}_{\text {Hubbard }}, T_{\text {tot }}^{(z)}\right]=\left[\mathcal{H}_{\text {Hubbard }}, T_{\text {tot }}^{2}\right]=0
$$

muestran la conservación de $T_{\text {tot }}^{(z)}$ y $T_{\text {tot }}^{2}$. En el caso de medio llenado, además de la componente $z$, conmutan las demás componentes $x, y$. La combinación las simetría de espín y pseudo-espín resulta en el grupo de simetría $\mathrm{SO}(4)$.

\subsubsection{Límites particulares}

En esta sección comentaremos brevemente dos límites particulares del modelo de Hubbard, los cuales conducen a dos de los modelos efectivos más ampliamente estudiados en materia condensada.

- El modelo $t-J$. En el límite de repulsión fuerte $(U / t \gg 1)$, podemos derivar un hamiltoniano efectivo que gobierna las excitaciones de baja energía. En este límite, los estados con doble ocupación (un electrón con espín $\uparrow$ y otro con espín $\downarrow$ en un mismo sitio) son energéticamente desfavorables, mientras que el principio de exclusión de Pauli previene que un electrón "salte" a un sitio vecino a menos que el electrón en el sitio vecino tenga índice de espín opuesto. La repulsión fuerte tiende a ordenar magnéticamente y obtenemos un sistema en que los electrones pueden moverse entre sitios, mientras que el término de repulsión deviene en un acoplamiento espín-espín antiferromagnético $J$. 
El hamiltoniano efectivo que se obtiene proyectando sobre el sub-espacio sin doble ocupación se conoce como el modelo $t-J$ y al orden más bajo en $t / U$ está dado por,

$$
\mathcal{H}_{t-J}=P_{s}\left(\mathcal{T}+\mathcal{H}+\mathcal{J}^{\prime}\right) P_{s}
$$

donde $P_{s}$ es un proyector en el sub-espacio de ocupación simple o nula (descartando la doble ocupación).

Los tres términos que sobreviven después de la proyección son,

- $\mathcal{T}$ es el término de hopping dado por

$$
\mathcal{T}=-\sum_{<i, j>, \sigma} t_{i j}\left(c_{i, \sigma}^{\dagger} c_{j, \sigma}+\text { h.c. }\right)
$$

- $\mathcal{H}$ es el hamiltoniano de Heisenberg con acoplamiento $J=\frac{4 t_{i j}^{2}}{U}$

$$
\mathcal{H}=\sum_{<i, j>} J_{i j}\left(\mathbf{S}_{i} \cdot \mathbf{S}_{j}-\frac{n_{i} n_{j}}{4}\right)
$$

donde el operador de espín en el sitio $j$ está definido como en la ecuación (1.21), - y finalmente $\mathcal{J}^{\prime}$

$$
\mathcal{J}^{\prime}=-\frac{1}{2 U} \sum_{i, j, k}^{i \neq j} t_{i j} t_{j k}\left[\sum_{\sigma}\left(c_{i, \sigma}^{\dagger} c_{k, \sigma} n_{j}\right)-c_{i}^{\dagger} \vec{\tau} c_{k} \cdot c_{j}^{\dagger} \vec{\tau} c_{j}\right]
$$

En la literatura, este último termino es frecuentemente omitido asumiendo que sus efectos son más pequeños en comparación con los del hopping [4].

Como se puede ver, el modelo $t-J$, incluye tanto la dinámica de los electrones en el término de hopping $\mathcal{T}$, como así también la interacción magnética entre los espines intrínsecos de estos. Como mencionamos antes, el hamiltoniano (1.28) corresponde al orden más bajo en $t / U$. La inclusión de o'rdenes superiores, introduce términos de hopping entre sitios más allá de primeros vecinos, interaciones magnéticas a segundos y terceros vecinos y términos de plaquetas, entre otros [17].

- Modelo de Heisenberg. El modelo $t-J$ tiene una característica particular a medio llenado (un electrón por sitio). En este caso, el proyector $P_{s}$ anula los términos $\mathcal{T}$ y el $\mathcal{J}^{\prime}$. El único que sobrevive a la proyección de $P_{s}$ es el que incluye la interacción magnética involucrada en el hamiltoniano $\mathcal{H}$, dando como resultado el modelo de Heisenberg cuántico,

$$
\begin{aligned}
\mathcal{H}_{\text {Heisenberg }} & =\sum_{<i, j>} J_{i j} \mathbf{S}_{i} \mathbf{S}_{j} \\
& =\sum_{<i, j>} J_{i j}\left(\frac{1}{2}\left(S_{i}^{+} S_{j}^{-}+S_{j}^{+} S_{i}^{-}\right)+S_{i, z} S_{j, z}\right)
\end{aligned}
$$


Así, en el límite de medio llenado, los grados de libertad de carga se "congelan" y como consecuencia se abre un gap en el espectro de las excitaciones electrónicas en el nivel de Fermi, mientras que el grado de libertad de espín permanece sin gap. Éste límite del modelo de Hubbard, se lo conoce como fase de aislador de Mott. A diferencia de los aisladores convencionales o aisladores de banda, el fenómeno es puramente cuántico y se debe únicamente al principio de exclusión de Pauli.

\subsubsection{Líquidos de Fermi y Luttinger}

En esta sección presentamos las características generales de dos conceptos muy importantes en física de materia condensada: líquido de Fermi y su contra parte líquido de Luttinger. Mientras que el primero está presente en la física de sistemas de electrones interactuantes en dos y en tres dimensiones espaciales; el segundo es un fenómeno puramente unidimensional $[8,18,19,20,21]$.

\section{Líquido de Fermi}

Landau desarrolló a finales de los años 50' la Teoría del líquido de Fermi [22].

Esta se ocupa de las propiedades de los sistemas fermiónicos de muchos cuerpos a bajas temperaturas ( $k_{B} T$ mucho menor que la energía de Fermi) en el estado normal, es decir, a temperaturas por encima de cualquier transición de fase que rompa alguna simetría (superconductividad, magnetismo, u otros). Esta teoría puede ser aplicada incluso en sistemas en donde la interacción entre los electrones sea del mismo órden de magnitud que el término cinético, donde la teoría estándar de perturbaciones no podría ser aplicada. Landau mostró que las propiedades macroscópicas de los metales en dos y tres dimensiones son muy similares a las de un gas de electrones libres, tal que la presencia de interacciones no produce cambios drásticos en las propiedades del sistema. En lugar de considerar los electrones reales, que son fuertemente interactuantes, la teoría de Landau considera partículas de carácter fermiónico que corresponden a electrones "vestidos" con fluctuaciones de densidad de carga. Estas partículas son denominadas cuasi-partículas y parte de la interacción es absorbida en la definición de sus parámetros (tales como su masa efectiva). Sin embargo, sigue existiendo una interacción residual entre las cuasi-partículas la cual queda descrita por medio de los denominados parámetros de Landau que no detallaremos aquí [22].

El concepto de cuasi-partículas tiene un cierto número de limitaciones, principalmente debido que el tiempo de vida media $\tau$ de las cuasi-partículas es finito. Sin embargo, para excitaciones cerca del nivel de Fermi uno tiene $1 / \tau \propto\left(\epsilon-\epsilon_{F}\right)^{2}$, es decir, el tiempo de vida media crece mientras más cerca del nivel de Fermi está su energía, y las cuasi-partículas de esta forma están bien definidas. En la práctica, esto significa que la teoría de Landau es muy usada para fenómenos a escalas de energías mucho más pequeñas que la energía de Fermi, pero inaplicable si esto no se cumple. En un gas de electrones libres a temperatura 

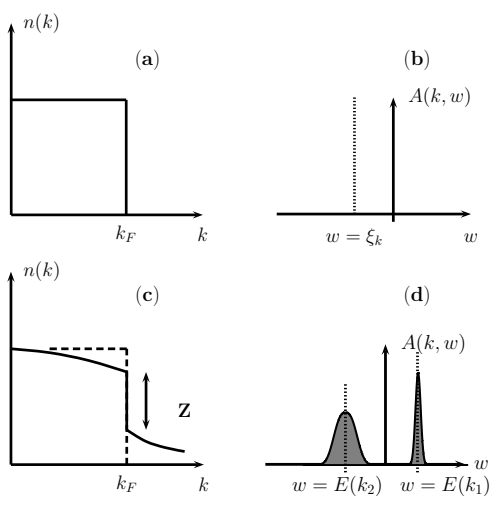

Figura 1.1: (a-b) Electrones libres: el número de ocupación $n_{k}$ tiene una discontinuidad de amplitud 1 en la superficie de Fermi. La función espectral $A(k, w)$ es una función delta, mostrando que las excitaciones poseen una relación frecuencia-momento $w(k)=\xi(k)$ bien definida. (c-d) Líquido de Fermi: el número de ocupación es esencialmente similar al gas de electrones libres, presentando una discontinuidad de magnitud $Z$ en el nivel de Fermi. Los picos anchos en la función espectral, revelan que las cuasi-partículas tienen un tiempo de vida medio finito. Figura extraída de Giamarchi [18].

cero, todos los estados dentro de la superficie de Fermi (estados con energía $\left.\epsilon(\mathbf{k})<\epsilon_{F}\right)^{1}$ se encuentran ocupados, mientras que todos aquellos fuera de la región están desocupados. La distribución $n(\mathbf{k})$ de fermiones libres con momento $\mathbf{k}$ obedece la estadística de Fermi-Dirac: a temperatura cero esta presenta una discontinuidad de amplitud 1 en el nivel de Fermi con $n(\mathbf{k})=1$ si $\epsilon(\mathbf{k})<\epsilon_{F}$, y cero en el otro caso (figura (1.1a)). Una excitación elemental del sistema, consiste en crear (respectivamente aniquilar) una partícula con momento bien definido $\mathbf{k}$ en un estado justo por encima (respectivamente por debajo) del nivel de Fermi. Tales excitaciones son llamadas de partículas (respectivamente huecos). Ellas son autoestados del hamiltoniano y por lo tanto su tiempo de vida media es infinito.

Para caracterizar estas excitaciones uno puede introducir la función espectral $A(\mathbf{k}, w)$ que es la probabilidad de encontrar un estado con frecuencia $w$ y momento k:

$$
A(\mathbf{k}, w)=-\frac{1}{\pi} \operatorname{Im} G_{r e t}(\mathbf{k}, w) .
$$

Para el caso de fermiones libres la función espectral corresponde a una distribución delta de Dirac (ver figura (1.1b)) indicando que los electrones libres tienen la relación frecuenciamomento $w=\xi_{\mathbf{k}}$ bien definida.

$$
A(\mathbf{k}, w)=-\pi \delta\left(w-\xi_{\mathbf{k}}\right), \quad \xi_{\mathbf{k}}=\epsilon(\mathbf{k})-\mu
$$

donde $\mu$ es el potencial químico.

En el caso interactuante, parte de la interacción entre las partículas originales se absorbe en la

\footnotetext{
${ }^{1} \epsilon(\mathbf{k})$ identifica la energía de partícula libre, $\xi_{\mathbf{k}}$ es la energía relativa al potencial químico $\mu$ y para el caso de partículas interactuantes la energía es $E(\mathbf{k})$
} 


\section{Modelo de Hubbard}

definición de las cuasi-partículas. Estas son tratadas como partículas con masa renormalizada por la interacción, que interactúan a través de una interacción residual. Al igual que en caso puramente libre, la distribución de momentos tiene una discontinuidad en la superficie de Fermi, sin embargo, la amplitud de esta discontinuidad es igual a un número $Z<1$ que disminuye a medida que se aumenta la interacción (ver figura (1.1c)) y se puede interpretar como la "fracción" del electrón que permanece en este estado de cuasi-partícula. La velocidad de las cuasi-partículas en la superficie de Fermi (la velocidad de Fermi) está dada por

$$
v_{F}=\left|\frac{\partial E(\mathbf{k})}{\partial \mathbf{k}}\right|_{\mathbf{k}=\mathbf{k}_{F}}
$$

La masa efectiva de la cuasi-partícula es entonces definida por $^{2}$

$$
v_{F}=\frac{\left|\mathbf{k}_{F}\right|}{m^{*}}
$$

En la vecindad de la superficie de Fermi, la energía de las cuasi-partículas toma la forma

$$
E(\mathbf{k}) \approx E\left(\mathbf{k}_{F}\right)+\frac{\left|\mathbf{k}_{F}\right|}{m^{*}}\left|\mathbf{k}-\mathbf{k}_{F}\right|
$$

La función espectral para cuasi-partículas aún exhibe picos como en el caso de fermiones libres, sin embargo estos presentan una distribución tipo Lorentziana (ver figura (1.1d)) centrada en $w=E(\mathbf{k})$ y con un ancho $1 / \tau$. Estos picos son más agudos para partículas con energías muy cercanas a $E_{F}$.

La función de Green para un fermión interactuante es:

$$
G(\mathbf{k}, w)=\frac{1}{\epsilon(\mathbf{k})-w+\Sigma(\mathbf{k}, w)}
$$

donde $\Sigma(\mathbf{k}, w)$ es la autoenergía originada a partir de la interacción entre los electrones. La autoenergía $\Sigma(\mathbf{k}, w)$ es una cantidad compleja cuya parte real modifica la energía $\epsilon(\mathbf{k}) \rightarrow$ $\epsilon(\mathbf{k})+\operatorname{Re} \Sigma(\mathbf{k}, w)$, la parte imaginaria $\operatorname{Im} \Sigma(\mathbf{k}, w)$, mide la inversa del tiempo de vida media $\tau^{-1}$ de los fermiones. La existencia de cuasi-partículas demanda que la autoenergía sea suficientemente pequeña como para poder ser expandida en la vecindad del nivel de Fermi. La función de Green es entonces reescrita cerca del nivel de Fermi permitiendo calcular $Z$. Un tratamiento más detallado de la teoría de Landau puede ser encontrado en $[18,22,23]$.

La pregunta que nos hacemos ahora es, ¿cuánto de la teoría de líquido de Fermi sobrevive en una dimensión?. Lo que veremos es que el concepto de cuasi-partícula no es válido en los sistemas electrónicos unidimensionales. Estos sistemas son descritos por un nuevo estado de la materia conocido como líquido de Luttinger

\footnotetext{
${ }^{2}$ A lo largo de la tesis vamos a tomar $\hbar=1$; de esta manera tenemos $v_{F}=\frac{\left|\mathbf{p}_{F}\right|}{m^{*}}=\frac{\hbar\left|\mathbf{k}_{F}\right|}{m^{*}} \equiv \frac{\left|\mathbf{k}_{F}\right|}{m^{*}}$
} 


\section{Líquido de Luttinger}

Sin realizar ningún cálculo es fácil anticipar que en los sistemas unidimensionales $(d=1)$, las interacciones tienen drásticas consecuencias en comparación con dimensiones más altas. En una dimensión, como se muestra de manera esquemática en la figura (1.2b), un electrón que trata de propagarse tiene que "empujar" sus vecinos debido a la interacción coulombiana. No es posible movimiento individual; cualquier excitación individual tiene que devenir en colectiva. Estas correlaciones entre las excitaciones son el origen de la mayor diferencia entre el mundo unidimensional y el de dimensiones más altas. En el caso de fermiones con espín,
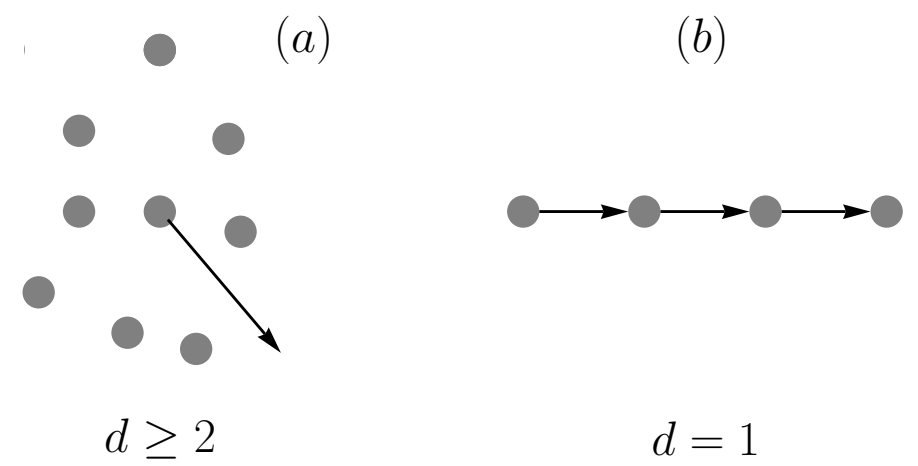

Figura 1.2: (a) En altas dimensiones, cerca de excitaciones cuasi-libres, estas partículas lucen como partículas individuales. (b) En una dimensión, en un sistema interactuante, un electrón no puede moverse sin empujar al resto de los electrones. Entonces, únicamente pueden existir excitaciones colectivas.

la situación es aún más sorprendente. Dado que únicamente pueden existir excitaciones colectivas, implica que una única excitación fermiónica tiene que separarse en una excitación colectiva llevando carga y otra llevando espín. Estas excitaciones tienen en general diferentes velocidades. Estas propiedades, completamente distintas a las del líquido de Fermi, son la esencia del líquido de Luttinger, y serán retomadas en la sección 2.1.4 cuando estudiemos con más detalle el modelo de Hubbard unidimensional.

Finalmente, vamos a mencionar una peculiaridad de las excitaciones del caso unidimensional, que no ocurre en dimensiones mayores. Comencemos analizando lo que ocurre en dimensión $d=2$. El componente crucial de las excitaciones de un gas de electrones es la denominada excitación hueco-partícula donde un electrón es tomado debajo del nivel de Fermi y promovido por encima de éste. Puesto que para crear una excitación de este estilo, es necesario destruir una partícula con momento $\mathbf{k}$ y crear una partícula con momento $\mathbf{k}+\mathbf{q}$, el momento de la excitación está bien definido y es igual a q. La energía de la excitación, por el contrario, depende de ambos k y q. De esta manera, la energía de la excitación hueco-partícula como función de $\mathbf{q}$ revela un continuo de energía. Así, para $|\mathbf{q}|<2\left|\mathbf{k}_{F}\right|$ uno puede crear una excitación de energía arbitraria al aniquilar una partícula en un punto justo por debajo de la superficie de Fermi y crear otra justo por arriba de la superficie de Fermi en otro punto, como se muestra en la figura 1.3a. 
Por el contrario, en $d=1$ la superficie de Fermi se reduce a dos puntos y uno no puede

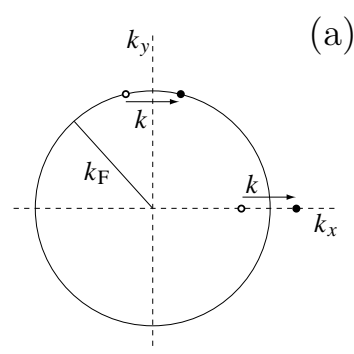

(a)

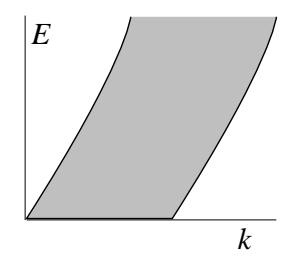

(b)
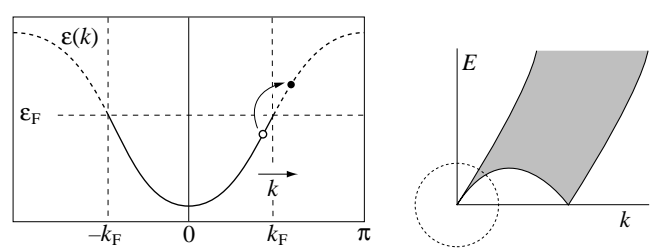

Figura 1.3: Espectro de excitaciones partícula-hueco para sistemas bi- y tri-dimensionales (a) y para uni-dimensionales (b). En una dimensión, al contrario de lo que ocurre en dimensiones más altas, las excitaciones partícula-hueco de baja energía, tienen restringidas la energía y el momento.

jugar con ángulos para incrementar el momento q sin pagar energéticamente. Puesto que la única manera de obtener excitaciones de baja energía es destruir y crear pares cerca de la "superficie" de Fermi, los únicos lugares donde la energía hueco-partícula puede tomar el valor cero es en $q=0$ y $q=2 k_{F}$. El comportamiento del espectro hueco-partícula en una dimensión es mostrado en la figura 1.3b. Dado el comportamiento lineal de la relación de dispersión de las partículas cerca del nivel de Fermi, los pares hueco-partícula poseen una dispersión muy aguda cerca de $q=0$ y de esta manera pueden propagarse coherentemente. En otras palabras, la partícula y el hueco tienen casi la misma velocidad de grupo a bajas energías y se propagan juntas. Cualquier débil atracción hueco-partícula es entonces obligada a tener dramáticos efectos, es decir, ligar el par en una entidad que se propaga de manera coherente: una nueva partícula.

No entraremos ahora más en detalle sobre la teoría de Líquido de Luttinger puesto que será estudiada con la técnica de bosonización en el capítulo 2.1.

\subsection{Modelo de Heisenberg}

En la sección 1.2.2, discutimos como el modelo de Heisenberg de espín $S=1 / 2$ emerge como un hamiltoniano efectivo para los aisladores de Mott, donde el operador de espín $\mathbf{S}$ 
puede ser representado en término de los operadores electrónicos. Sin embargo, uno puede concebir situaciones donde el espín $\mathbf{S}_{j}$ situado en el sitio $j$ es realizado por un objeto diferente (en particular por un átomo con momento magnético no nulo) cuya magnitud puede ser mayor a $S=1 / 2$. Para los propósitos de nuestra discusión, no necesitamos especificar la realización concreta del espín.

Una cuestión central en el estudio de sistemas fuertemente correlacionados es la clasificación de todas las posibles fases aisladoras de Mott a temperatura cero. La estrategia general para describir las posibles fases asociadas con los grados de libertad de espín (denominado magnetismo cuántico) de los aisladores de Mott consiste en analizar modelos de espines localizados tales como el modelo de Heisenberg antiferromagnético:

$$
\mathcal{H}=\sum_{<i, j>} J_{i j} \mathbf{S}_{i} \cdot \mathbf{S}_{j}+\ldots
$$

La notación $\langle i, j>$, significa que en la suma solo intervienen términos conectados por sitios vecinos $i, j$ con un acoplamiento antiferromagnético $J_{i j}>0$. El modelo de Heisenberg es un modelo cuántico, donde $\mathbf{S}_{i}$ es el operador de espín en el sitio $i(i=1, \ldots \mathcal{N}$ sitios $)$ con componentes $\left(S_{i}^{x}, S_{i}^{y}, S_{i}^{z}\right)$ que satisfacen el álgebra de $\mathrm{SU}(2)$,

$$
\left[S_{j}^{a}, S_{k}^{b}\right]=i \varepsilon^{a b c} S_{k}^{c} \delta_{j k}
$$

donde $\in^{a b c}$ es el tensor completamente antisimétrico.

Los puntos ... representan términos adicionales que pueden ser tenidos en cuenta, como interacción a segundos vecinos o interacción de varios espines (conocida como ring exchange). En general, el hamiltoniano (1.39) es un modelo fundamental tanto para el magnetismo cuántico, como para otros fenómenos que pueden ser descritos de manera efectiva por operadores cuánticos de espín.

$\mathcal{H}$ es invariante $\mathrm{SU}(2)$ (es decir, invariante frente a una rotación de espín global) dado que conmuta con cada de las tres componentes del espín total,

$$
\mathbf{S}_{\text {total }}=\sum_{i=1}^{\mathcal{N}} \mathbf{S}_{i}
$$

Entonces, los autoestados de $\mathcal{H}$ se deben encontrar en subespacios de la forma

$$
\begin{aligned}
\Psi & =\left|S_{\text {total }}, M, \ldots\right\rangle \\
S_{\text {total }} & \leq \mathcal{N} S, \\
M & =-S_{\text {total }},-S_{\text {total }}+1, \ldots, S_{\text {total }}
\end{aligned}
$$

donde $M$ es el autovalor de la magnetización total $S_{\text {total }}^{z}$ y

$$
\mathbf{S}_{\text {total }}^{2}=S_{\text {total }}\left(S_{\text {total }}+1\right)
$$

Además del modelo cuántico, podemos obtener "el modelo clásico" si consideramos $\mathbf{S}$ como 
un vector clásico. El modelo clásico "es limitado" para sistemas con espines grandes. Esto se puede ver al reescribir $\mathbf{S}_{i}$ en términos de un operador de espín normalizado $\boldsymbol{\Omega}_{\mathbf{i}}, \mathbf{S}=$ $S(S+1) \boldsymbol{\Omega}_{\mathbf{i}}$, con lo cual (1.40) queda

$$
\left[\Omega_{j}^{a}, \Omega_{k}^{b}\right]=\frac{i \in^{a b c}}{S(S+1)} \Omega_{k}^{c} \delta_{j k} .
$$

Tomar el límite de espín grande $S \rightarrow \infty$, conduce a que los operadores de espín normalizados conmuten y de esta manera pueden ser considerados como vectores clásicos. Por lo general, uno usa éste límite para contrastar otras técnicas al menos para valores grandes del espín.

El fácil verificar que el estado fundamental del modelo de Heisenberg clásico ferromagnético $\left(J_{i j}<0\right.$ en ecuación (1.39)), se consiste en la configuración con todos los espines apuntando en una misma dirección (ver figura 1.4a); cualquier vector que apunte en otra dirección que no sea la del resto aumenta la energía.

En el caso cuántico, el estado construido a partir del producto directo de los autoestados $|\uparrow\rangle_{i}$ del operador $S_{i}^{z}$ en el sitio $i$ con mayor autovalor $S_{i}^{z}|\uparrow\rangle_{i}=\frac{1}{2}|\uparrow\rangle_{i}$ dado por

$$
|\psi\rangle_{0}^{\text {Ferro }}=|\uparrow\rangle_{1} \otimes \ldots \otimes|\uparrow\rangle_{\mathcal{N}}
$$

es realmente un autoestado del hamiltoniano y corresponde al estado fundamental.

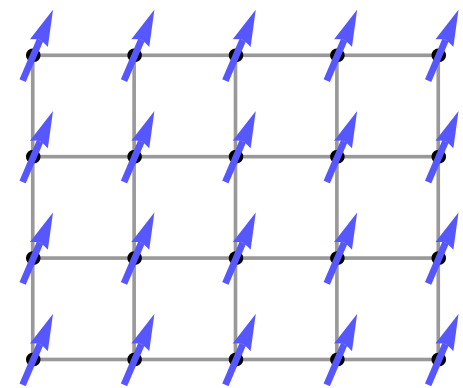

(a)

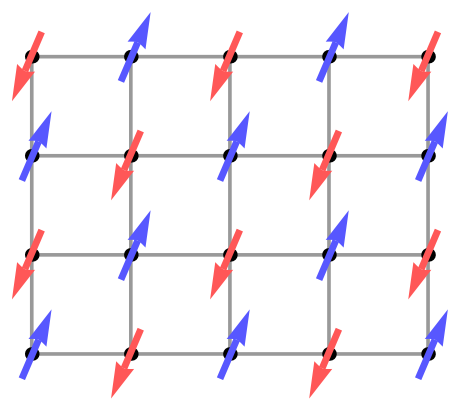

(b)

Figura 1.4: Configuraciones del estado fundamental del modelo de Heisenberg clásico en una red cuadrada, (a) ferromagnético y (b) antiferromagnético.

El caso antiferromagnético es un problema mucho más complicado, aún el límite clásico. Como ejemplo, consideremos el hamiltoniano de Heisenberg clásico en una red cuadrada donde los acoplamientos $J_{i j}>0$ más importantes son únicamente primeros vecinos. En ese caso, el estado fundamental clásico corresponde a la configuración donde todos los sitios vecinos tienen espines (vectores) apuntando en direcciones opuestas (ver figura 1.4b). Este estado es llamado estado de Néel, después de que L. Néel en 1932 [24], propuso la posibilidad orden antiferromagnético.

En cuanto al modelo cuántico, se puede construir una configuración análoga a la clásica dada por

$$
|\psi\rangle_{0}^{\text {AntiFerro }}=|\uparrow\rangle_{1} \otimes|\downarrow\rangle_{2} \otimes|\uparrow\rangle_{3}, \ldots \otimes|\downarrow\rangle_{\mathcal{N}}
$$


sin embargo, esta configuración no es más ya un autoestado del hamiltoniano. Podemos ver directamente el problema con el ejemplo de dos espines. En efecto, introduciendo los operadores escalera $S_{i}^{ \pm}=S^{x} \pm i S^{y}$, y escribiendo

$$
2 \mathbf{S}_{i} \cdot \mathbf{S}_{j}=\left(S_{i}^{+} S_{j}^{-}+S_{i}^{-} S_{j}^{+}\right)+2 S_{i}^{z} S_{j}^{z}
$$

es evidente que no es un autoestado porque

$$
2 \mathbf{S}_{i} \cdot \mathbf{S}_{j}|\uparrow\rangle_{i} \otimes|\downarrow\rangle_{j}=|\downarrow\rangle_{i} \otimes|\uparrow\rangle_{j}-\frac{1}{2}|\uparrow\rangle_{i} \otimes|\downarrow\rangle_{j} .
$$

\subsubsection{Origen microscópico de las interacciones magnéticas}

En esta sección discutiremos brevemente algunos tipos de interacciones magnéticas que son responsables de las propiedades que se observan en un gran número de materiales. Estas propiedades se basan en el hecho que los momentos magnéticos interactúan con cada uno de sus vecinos, dando como resultado, que el sistema pueda adquirir orden de largo alcance. La primera interacción es la "interacción de intercambio". Esta es consecuencia de un efecto puramente cuántico y se debe a la interacción de Coulomb y el principio de exclusión de Pauli. La situación en la cual dos electrones en átomos magnéticos vecinos interactúan de manera directa se conoce como "Interacción de intercambio directo" porque la interacción se da sin la necesidad de átomos intermediarios.

Por el contrario, la "Interacción de intercambio indirecto" entre dos átomos magnéticos es mediada por un átomo no magnético. Si el solapamiento de las funciones de onda involucradas es pequeño (por ejemplo, átomos metálicos de tierras raras con electrones $4 f$ localizados) entonces el acoplamiento por intercambio directo no es el mecanismo dominante para las propiedades magnéticas. Para esta clase de sistemas el acoplamiento de "intercambio indirecto" es responsable del magnetismo. El tipo de "intercambio indirecto" depende significativamente de la clase de material magnético. Así, podemos identificar tres tipos:

- Interacción de súper intercambio:

Este tipo de interacción ocurre en iones sólidos. La interacción de intercambio ocurre entre iones magnéticos que no son vecinos, es mediada por medio de iones no magnéticos que se encuentran localizados entre ellos.

- Interacción de intercambio RKKY:

La interacción RKKY (Ruderman, Kittel, Kasuya, Yosida) ocurre en metales con momentos magnéticos localizados. El intercambio es mediado por los electrones de valencia. El acoplamiento es caracterizado por la dependencia con la distancia de la integral de intercambio $J_{R K K Y}(r)$ :

$$
J_{R K K Y}(r) \propto F\left(2 k_{F} r\right)
$$

con

$$
F(x)=\frac{\sin x-x \cos x}{x^{4}}
$$


Este tipo de acoplamiento es de largo alcance y anisotrópico que frecuentemente resulta en arreglos complicados de espines. Además, éste posee un comportamiento oscilatorio. Entonces, el tipo de acoplamiento Ferro- o Antiferromanético, surge en función de la distancia entre momentos magnéticos. Un ejemplo es representado por los metales de tierras raras con sus electrones localizados en las bandas $4 f$.

- Interacción de doble intercambio:

En algunos óxidos los iones magnéticos mezclan valencias, es decir se presentan diferentes estados de oxidación que resulta en un arreglo ferromagnético. Un ejemplo corresponde a la manganíta $\left(\mathrm{Fe}_{3} \mathrm{O}_{4}\right)$ que incluye tanto iones $\mathrm{Fe}^{2+}$ como $\mathrm{Fe}^{3+}$.

\subsubsection{Orden de largo alcance: teorema de Mermin-Wagner}

En esta sección mencionamos brevemente el rol de la temperatura en los estados magnéticamente ordenados de espines. Para ello necesitamos introducir lo que se conoce como función de correlación. La función de correlación es una cantidad que determina si alguna variable específica del sistema, presenta algún tipo de orden. Así, en el caso de un sistema de $N$ espines a temperatura $T$, en un campo magnético externo de ordenamiento $h$ el hamiltoniano $\mathcal{H}(h)$ está dado por

$$
\mathcal{H}(h)=\sum_{\langle i j\rangle} J_{i j} \mathbf{S}_{\mathbf{r}_{i}} \cdot \mathbf{S}_{\mathbf{r}_{i}}-h \sum_{i} S_{\mathbf{r}_{i}}^{z},
$$

la función de correlación espín-espín a campo cero (o simplemente correlación espín-espín) está dada por

$$
\begin{aligned}
G\left(\mathbf{r}_{i}, \mathbf{r}_{j}\right) & =\left\langle\mathbf{S}_{\mathbf{r}_{i}} \cdot \mathbf{S}_{\mathbf{r}_{j}}\right\rangle \\
& =\lim _{h \rightarrow 0} \lim _{N \rightarrow \infty} \frac{1}{\mathcal{Z} N} \operatorname{Tr}\left[e^{-\mathcal{H}(h) / T} \mathbf{S}_{\mathbf{r}_{i}} \cdot \mathbf{S}_{\mathbf{r}_{j}}\right],
\end{aligned}
$$

donde $\mathcal{Z}=\operatorname{Tr}\left[e^{-\mathcal{H}(h) / T}\right]$. Dependiendo del comportamiento de la correlación $\left\langle\mathbf{S}_{\mathbf{r}_{i}} \cdot \mathbf{S}_{\mathbf{r}_{j}}\right\rangle$, el sistema puede presentar orden de largo alcance, cuasi orden de largo alcance o finalmente ser desordenado magnéticamente. Así,

- si el $\lim _{\left|\mathbf{r}_{i}-\mathbf{r}_{j}\right| \rightarrow \infty}\left\langle\mathbf{S}_{\mathbf{r}_{i}} \cdot \mathbf{S}_{\mathbf{r}_{j}}\right\rangle \neq 0$, el sistema presenta orden de largo alcance. En ese caso, el parámetro de orden magnético (la magnetización escalonada con vector de ordenamiento $\mathbf{q}$ )

$$
\begin{aligned}
\lim _{h \rightarrow 0} m(\mathbf{q}, h)= & \lim _{h \rightarrow 0} \lim _{N \rightarrow \infty} \frac{1}{\mathcal{Z} N} \operatorname{Tr}\left[e^{-\mathcal{H}(h) / T} S_{\mathbf{q}}^{z}\right] \\
\text { donde } & S_{\mathbf{q}}^{z}=\sum_{i} e^{i \mathbf{q} \cdot \mathbf{r}_{i}} S_{\mathbf{r}_{i}}^{z}
\end{aligned}
$$

es no nulo. 
- si $\lim _{\left|\mathbf{r}_{i}-\mathbf{r}_{j}\right| \rightarrow \infty}\left\langle\mathbf{S}_{\mathbf{r}_{i}} \cdot \mathbf{S}_{\mathbf{r}_{j}}\right\rangle=0$ como una ley de potencias,

$$
\left.\left\langle\mathbf{S}_{\mathbf{r}_{i}} \cdot \mathbf{S}_{\mathbf{r}_{j}}\right\rangle\right|_{\left|\mathbf{r}_{i}-\mathbf{r}_{j}\right|>>0} \sim \frac{1}{\left|\mathbf{r}_{i}-\mathbf{r}_{j}\right|^{\alpha}}
$$

se dice que el sistema presenta cuasi-orden de largo alcance.

- finalmente, si $\lim _{\left|\mathbf{r}_{i}-\mathbf{r}_{j}\right| \rightarrow \infty}\left\langle\mathbf{S}_{\mathbf{r}_{i}} \cdot \mathbf{S}_{\mathbf{r}_{j}}\right\rangle=0$ con un decaimiento tipo exponencial, el sistema presenta orden de corto alcance o es desordenado magnéticamente.

Es de esperar que los estados excitados térmicamente reduzcan las correlaciones entre espines y que para $k_{B} T$ mucho mayor que el rango de energías característico del sistema $(J)$ los espines se descorrelacionen a grandes distancias y se anule el parámetro de orden. Existirá entonces una temperatura $T_{c}$, por encima de la cual se produce una transición entre una fase ordenada y una desordenada. Sin embargo, la dimensionalidad del sistema tiene un drástico efecto sobre esta ruptura de simetría. Mermin y Wagner [25], demostraron que en modelos uni- y bidimensionales con interacciones de corto alcance, cuyos estados fundamentales rompen alguna simetría continua del hamiltoniano, la temperatura crítica es estrictamente igual a cero. Es decir el orden de largo alcance es inestable, para todo $S$, ante la presencia de fluctuaciones térmicas.

\section{Teorema de Mermin- Wagner:}

Para el modelo cuántico de Heisenberg (ec. 1.52) con interacciones de corto alcance que obedecen

$$
\bar{J}=\frac{1}{2 N} \sum_{i j}\left|J_{i j}\right|\left|\mathbf{x}_{i}-\mathbf{x}_{j}\right|^{2}<\infty,
$$

no puede haber ruptura espontánea de simetría en una y dos dimensiones a $T>0$, esto es

$$
\lim _{h \rightarrow 0} m(\mathbf{q}, h)=0
$$

El resultado principal que se obtiene (ver referencia [4]) es una cota superior para la magnetización en dimensión $d$ como función de la temperatura $T$ y el campo de ordenamiento $h$ : Para $h$ cerca de cero, en $d=1$, se tiene:

$$
m(\mathbf{q}, h) \leq \operatorname{cte} \frac{S(S+1) \bar{J}^{1 / 3}}{T^{2 / 3}} h^{1 / 3}
$$

que se anula cuando $h \rightarrow 0$ y $T>0$, mientras que para $d=2$ :

$$
m(\mathbf{q}, h) \leq \operatorname{cte} \frac{S(S+1) \bar{J}^{1 / 2}}{T^{1 / 2}} \frac{1}{\sqrt{|\ln (h)|}}
$$

que también se anula para $h \rightarrow 0$ y $T>0$. Para el caso de $d=3$, un resultado similar conduce a la posibilidad de encontrar una temperatura finita para la cual se satisface la 


\section{Modelo de Heisenberg}

desigualdad. Por lo tanto, la ruptura espontánea de simetría y la aparición de una fase ordenada es esperada en $d=3$ a temperatura $T>0$ para el hamiltoniano de Heisenberg, lo cual se observa en sistemas reales.

El teorema de Mermin-Wagner no se aplica a $T=0$, sin embargo las fluctuaciones cuánticas juegan un rol similar en cuanto a que compiten con el orden magnético.

Un resultado importante es que si el espectro de excitaciones presenta un gap, esto es

$$
E_{m}-E_{0}>\Delta, \forall m \neq 0
$$

donde $\Delta$ es independiente de $h$ y $N$, el estado fundamental del modelo de Heisenberg es desordenado. Como ejemplo, la cadena de espín entero antiferromagnética exhibe el gap de Haldane en el espectro de excitaciones. Los modelos que poeen gap, no poseen verdadero orden de largo alcance en su estado fundamental. El análisis del modelo sigma no lineal predice que las funciones de correlación decaen exponencialmente a largas distancias [4].

La implicación inversa (excitaciones sin gap presentan verdadero orden de largo alcance) no es cierta. Por ejemplo el modelo de Heisenberg para la cadena de espín semi-entero antiferromagnético, presenta un espectro de excitaciones sin gap sin embargo no hay verdadero orden de largo alcance puesto que las funciones de correlación decaen como ley de potencias.

\subsubsection{Sistemas desordenados y líquidos de espines}

Un tema central de los últimos años dentro del magnetismo cuántico, comenzando en la propuesta de Anderson [26], ha sido la búsqueda de los líquidos de espines (SL), es decir, fases sin orden magnético del tipo Néel de largo alcance. Es de sospechar que las fases SL se estabilicen a bajas dimensiones o en presencia de frustración (ver sección 1.3.4), es decir, en situaciones donde las fluctuaciones cuánticas puedan suprimir fuertemente el magnetismo. En este marco se espera una física muy rica con excitaciones exóticas de baja energía que requiere, en la mayoría de los casos, el uso de técnicas no perturbativas para determinar completamente sus propiedades.

En una dimensión, a $T=0$ las fluctuaciones cuánticas tienden a desordenar el estado fundamental. De esta manera, en una dimensión se espera que el estado fundamental esté en una fase SL. Sin embargo, esto no sucede siempre y las propiedades del estado fundamental dependen del modelo estudiado. Como ejemplo podemos mencionar dos casos muy estudiados: el hamiltoniano de Heisenberg con acoplamientos a primeros vecinos y el hamiltoniano de Majumdar-Ghosh (ambos de espín $S=1 / 2$ ),

$$
\begin{aligned}
\mathcal{H}_{J} & =J \sum_{i=1}^{N} \mathbf{S}_{i} \cdot \mathbf{S}_{i+1} \\
\mathcal{H}_{M G} & =J \sum_{i=1}^{N}\left(\mathbf{S}_{i} \cdot \mathbf{S}_{i+1}+\frac{1}{2} \mathbf{S}_{i} \cdot \mathbf{S}_{i+2}\right)
\end{aligned}
$$


En el primer caso, el estado fundamental no presenta orden de largo alcance y corresponde a un líquido de Luttinger. En este caso la función de correlación decae como una ley de potencias de la forma,

$$
\left\langle\mathbf{S}_{r} \cdot \mathbf{S}_{0}\right\rangle \sim-\frac{1}{4 \pi r^{2}}+(-1)^{r / a} \frac{\text { cte }}{r}
$$

mientras que el espectro de excitaciones no posee gap.

En el segundo caso, el estado fundamental es doblemente degenerado y las excitaciones presentan un gap en su espectro. Estos estados están formados por productos de singletes de la forma

$$
|G S\rangle_{ \pm}=\frac{1}{2^{N / 2}} \prod_{n=1}^{N / 2}\left(|\uparrow\rangle_{2 n}|\downarrow\rangle_{2 n \pm 1}-|\downarrow\rangle_{2 n}|\uparrow\rangle_{2 n \pm 1}\right)
$$

Se puede verificar que ninguno de los estados presentan orden magnético calculando la correlación espín-espín; sin embargo (1.62) posee otro tipo de orden conocido como orden de dímeros. Este tipo de orden se puede observar a partir de otro tipo de función de correlación conocida como correlación dímero-dímero.

El operador de dímero entre un par de sitios $(i, j)$ está dado por

$$
d_{\mathbf{r}_{i}, \mathbf{r}_{j}}=\frac{\left(1-P_{\mathbf{r}_{i}, \mathbf{r}_{j}}\right)}{2}
$$

donde $P_{\mathbf{r}_{i}, \mathbf{r}_{j}}=2\left(\mathbf{S}_{\mathbf{r}_{i}} \cdot \mathbf{S}_{\mathbf{r}_{j}}+\frac{1}{4}\right)$ es el operador de permutación. Con esta definición, en un estado como (1.62), el operador de dímero vale 1 si entre los sitios $(i, j)$ hay formado un singlete, y $1 / 4$ entre los demás sitios.

La correlación dímero-dímero entre un par de sitios de referencia $(i, j)$ y otro par de sitios $(k, l)$ es

$$
p_{\left(\mathbf{r}_{i}, \mathbf{r}_{j} ; \mathbf{r}_{k}, \mathbf{r}_{l}\right)}=\frac{\left\langle d_{\mathbf{r}_{i}, \mathbf{r}_{j}} d_{\mathbf{r}_{k}, \mathbf{r}_{k}}\right\rangle-\left\langle d_{\mathbf{r}_{i}, \mathbf{r}_{j}}\right\rangle\left\langle d_{\mathbf{r}_{k}, \mathbf{r}_{l}}\right\rangle}{\left\langle d_{\mathbf{r}_{i}, \mathbf{r}_{j}}\right\rangle\left(1-\left\langle d_{\mathbf{r}_{k}, \mathbf{r}_{l}}\right\rangle\right)}
$$

Si $p_{\left(\mathbf{r}_{i}, \mathbf{r}_{j} ; \mathbf{r}_{k}, \mathbf{r}_{l}\right)}=1$ la presencia de un singlete entre los sitios $(i, j)$ implica la existencia de un singlete entre $(k, l)$; si $p_{\left(\mathbf{r}_{i}, \mathbf{r}_{j} ; \mathbf{r}_{k}, \mathbf{r}_{l}\right)}=0$ hay una ausencia de correlaciones entre singletes entre los pares de sitios $(i, j)$ y $(k, l)$. Si $p_{\left(\mathbf{r}_{i}, \mathbf{r}_{j} ; \mathbf{r}_{k}, \mathbf{r}_{l}\right)}<0$, un singlete en el par de sitios $(i, j)$ induce una tendencia de correlación ferromagnética entre los sitios $(k, l)$. A modo de comparación, en la figura (1.5) se muestran funciones de correlación espín-espín (círculos) y dímero-dímero (cuadrados) calculadas en el estado fundamental del hamiltoniano de Majumdar-Ghosh. Se puede observar que el estado fundamental no presenta orden magnético de largo alcance, mientras que sí posee orden de dímeros de largo alcance.

Desde el punto de vista técnico, el caso unidimensional es extremadamente favorable puesto que existen muchas herramientas no perturbativas para caracterizar completamente las propiedades físicas de diferentes SL. Entre ellas podemos nombrar, teorías de campos conformes [28, 29], bosonización [30], y cálculos numéricos tales como grupo de renormalización de la matriz densidad (DMRG) y diagonalización exacta. 


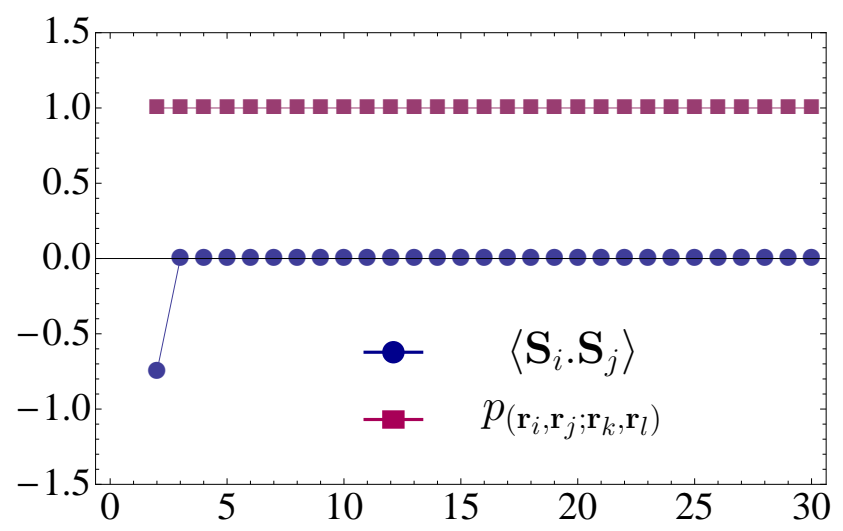

Figura 1.5: Funciones de correlación espín-espín (círculos) y dímero-dímero (cuadrados) calculadas mediante diagonalización exacta en el estado fundamental del hamiltoniano de Majumdar-Ghosh.

La determinación de las propiedades de los líquidos de espines unidimensionales no es un problema puramente académico puesto que muchas realizaciones experimentales de cadenas de espines han sido sintetizadas en los últimos años [31, 32].

En el caso de dos dimensiones, se han encontrado sistemas que no presentan orden magnético, mostrando otros tipos de órdenes como el de dímeros, antes mencionado. Sin embargo, la comprensión de los sistemas magnéticos cuánticos bidimensionales es mucho más limitada que en el caso unidimesnional. El número de resultados experimentales inexplicables es extremadamente grande, y lamentablemente las herramientas teóricas son bastantes limitadas. Entre las técnicas más confiables para estudiar sistemas en dos dimensiones, se pueden nombrar por el lado de las técnicas analíticas: el método de ondas de espín lineal, la teorías de campo medio de representaciones bosónicas (campo medio de bosones de Schwinger), fermiónicas (campo medio de fermiones de Abrikosov), de representanciones mixtas, generalizaciones tipo Large- $\mathcal{N}$ y modelos efectivos de teorías de campo. Por el lado de técnicas numéricas podemos nombrar expansiones en serie, diagonalización exacta, Montecarlo cuántico.

Además de los resultados obtenidos de los métodos citados, se encuentra en la literatura que mediante diversos métodos complementarios uno encuentra al menos cuatro clases de física distinta en sistemas en dos dimensiones:

- Fases semi-clásicas tipo Néel

- y tres tipos de de fases "puramente cuánticas" basadas en la formación de singletes.

Las propiedades de estas cuatro fases son resumidas en la tabla (1.1). Las fases cuánticas aparecen en situaciones donde hay interacciones competitivas, un alto grado de frustración magnética y un número de coordinación bajo.

Este tema lo volveremos a tratar en el capítulo 5 cuando estudiemos el modelo de Heisenberg en redes bidimensionales. 


\begin{tabular}{|c|c|c|c|}
\hline Fases & $\begin{array}{c}\text { Simetría rota } \\
\text { en el } \\
\text { fundamental }\end{array}$ & $\begin{array}{c}\text { Parámetro } \\
\text { de orden }\end{array}$ & Excitaciones \\
\hline \hline $\begin{array}{c}\text { Semi-clásico, } \\
\text { Orden de Néel }\end{array}$ & $\begin{array}{c}\text { SU(2) } \\
\text { Grupo espacial } \\
\text { Inversión temporal }\end{array}$ & $\begin{array}{c}\text { Magnetización } \\
\text { escalonada }\end{array}$ & Magnones sin gap \\
\hline $\begin{array}{c}\text { Valence Bond } \\
\text { Crystal }\end{array}$ & Grupo espacial & $\begin{array}{c}\text { Dímero-Dímero LRO } \\
\text { o plaquetas S=0 } \\
\text { LRO }\end{array}$ & $\begin{array}{c}\text { Excitaciones con gap, } \\
\text { Espinones confinados }\end{array}$ \\
\hline $\begin{array}{c}\text { Líquido de espines } \\
\text { R.V.B. } \\
\text { (Type I) }\end{array}$ & $\begin{array}{c}\text { Degeneración } \\
\text { topológica }\end{array}$ & $\begin{array}{c}\text { Parámetro de orden } \\
\text { no local }\end{array}$ & $\begin{array}{c}\text { Excitaciones con gap } \\
\text { Espinones deconfinados }\end{array}$ \\
\hline $\begin{array}{c}\text { Líquido de espines } \\
\text { R.V.B. }\end{array}$ & $\begin{array}{c}\text { Degeneración } \\
\text { topológica }\end{array}$ & $\begin{array}{c}\text { Parámetro de orden } \\
\text { no local }\end{array}$ & $\begin{array}{c}\text { Excitaciones sin gap } \\
\text { tipo singlete, } \\
\text { Gap en excitaciones } \\
\text { de tripletes, }\end{array}$ \\
& & & $\begin{array}{c}\text { Espinones deconfinados } \\
\text { Entropía a T=0 }\end{array}$ \\
\hline
\end{tabular}

Cuadro 1.1: Posibles fases de sistemas magnéticos bidimensionales. Tabla extraída de Claire Lhuillier, Gregoire Misguich arXiv:cond-mat/0109146v1.

\subsubsection{Sistemas frustrados}

El concepto de frustración fue definido por Villain y Toulouse [33, 34] a finales de años 70 's en el contexto de vidrios de espín, que marcaron el comienzo de una extensa investigación de los sistemas frustrados hasta la actualidad. La frustración puede deberse a dos posibles causas:

- Interacciones competitivas

- Frustración geométrica

\section{Frustración geométrica}

Para simplificar el problema, vamos a comenzar analizando el hamiltoniano de espines de Ising para entender la idea de frustración. El hamiltoniano de Ising de dos espines está dado por

$$
\mathcal{H}_{\text {Ising }}=J S_{1}^{z} \cdot S_{2}^{z}
$$

Dado que en el hamiltoniano de Ising solo intervienen las componentes $z$ de los operadores de espín, éste es diagonal en la base canónica del espacio de espín, $\left\{\left|\sigma_{1}\right\rangle \otimes \ldots \otimes\left|\sigma_{N}\right\rangle\right\}$ 
$\left(\left|\sigma_{i}\right\rangle=\uparrow, \downarrow\right)$, y por lo tanto las variables de espín se pueden representan por cantidades escalares de magnitud $1^{3}$.

Si la constante de interacción es negativa $J<0$ (caso ferromagnético), el mínimo de la energía corresponde a la configuración en la cual ambos espines son paralelos ( toman el mismo valor de $S^{z}$ ) y su valor es $E=-|J|$. Si por el contrario, $J$ es positivo (interacción antiferromagnética), el mínimo de la energía corresponde a la configuración donde los espines son antiparalelos.

Consideremos el caso de un sistema de tres espines acoplados ferromagnéticamente como se muestra en la figura 1.6 - Es fácil ver que en el sistema de 3 sitios con interacciones ferro-

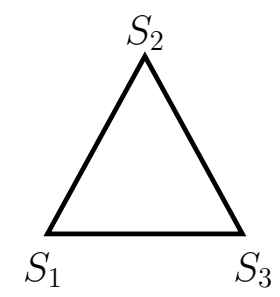

Figura 1.6: Arreglo de tres espines.

magnéticas, el estado fundamental corresponde a la configuración en que todos los espines están paralelos. Cada par de espines en interacción toman la configuración cuya energía es la misma que la del sistema de dos espines. En este caso se dice que la interacción de cada par está completamente satisfecha. Esta propiedad se cumple para cualquier tipo de red, siempre y cuando los acoplamientos sean ferromagnéticos.

Si $J>0$ (caso antiferromagnético), se puede ver que no hay manera de que todos los pares de acoplamientos sean satisfechos. El estado fundamental es seis veces degenerado correspondiente a las configuraciones

$$
\begin{aligned}
|G S\rangle_{a} & =|\uparrow\rangle_{1} \otimes|\uparrow\rangle_{2} \otimes|\downarrow\rangle_{3} \\
|G S\rangle_{b} & =|\uparrow\rangle_{1} \otimes|\downarrow\rangle_{2} \otimes|\uparrow\rangle_{3} \\
|G S\rangle_{c} & =|\downarrow\rangle_{1} \otimes|\uparrow\rangle_{2} \otimes|\uparrow\rangle_{3} \\
|G S\rangle_{d} & =\left.|G S\rangle_{a}\right|_{\uparrow \leftrightarrow \downarrow} \\
|G S\rangle_{e} & =\left.|G S\rangle_{b}\right|_{\uparrow \leftrightarrow \downarrow} \\
|G S\rangle_{f} & =|G S\rangle_{c} \mid \uparrow \leftrightarrow \downarrow
\end{aligned}
$$

con una energía $E_{0}=-J$. En este caso se dice que el sistema está frustrado.

En el caso del hamiltoniano de Heisenberg (operadores de espín con tres componentes), la situación es muy similar. En el caso de 2 espines con $S=1 / 2$ acoplados ferromagnéticamente, la energía del estado fundamental es $E_{0}=-\frac{|J|}{4}$ (para $J<0$ ) y el estado tiene la configuración $|\uparrow\rangle_{1} \otimes|\uparrow\rangle_{2}$. En el caso antiferromagnético, la energía es $E_{0}=-\frac{3 J}{4}$ y el estado

\footnotetext{
${ }^{3}$ La elección los valores de $S_{i}^{z}= \pm 1$ es estandar y cualquier otra elección solo modifica mediante una constante al hamiltoniano.
} 
corresponde al singlete $\frac{1}{\sqrt{2}}\left(|\uparrow\rangle_{1} \otimes|\downarrow\rangle_{2}-|\downarrow\rangle_{1} \otimes|\uparrow\rangle_{2}\right)$.

En el caso de 3 espines acoplados ferromagnéticamente, la energía del estado fundamental es $E_{0}=-\frac{3|J|}{4}$ siendo esta la energía de los tres enlaces satisfechos. En el caso antiferromagnético, el estado fundamental es cuatro veces degenerado con una energía $E_{0}=-\frac{3 J}{4}$. Una de las 4 posible configuraciones es

$$
\frac{1}{\sqrt{2}}\left(|\uparrow\rangle_{1} \otimes|\downarrow\rangle_{2}-|\downarrow\rangle_{1} \otimes|\uparrow\rangle_{2}\right) \otimes|\uparrow\rangle_{3}
$$

Así, el efecto de la frustración sobre los espines del triángulo es el de acomodar en estado singlete entre dos de ellos dejando libre el tercero.

La combinación de frustración y efectos cuánticos hace a estos sistemas grandes candidatos para encontrar física no convencional. Sin embargo, la frustración geométrica por si sola no es suficiente para desestabilizar el orden magnético. El primer ejemplo de un modelo frustrado y que presenta orden magnético, corresponde al modelo de Heisenberg en la red triangular. Una gran cantidad de trabajos analíticos y numéricos [35] han revelado que el sistema presenta un orden tipo Néel como se muestra en la figura 1.7. Este orden, es análogo al que presenta el modelo clásico de Heisenberg sobre la red triangular lo cual indica que las fluctuaciones cuánticas no son suficientemente grandes como para desestabilizarlo en el modelo cuántico.

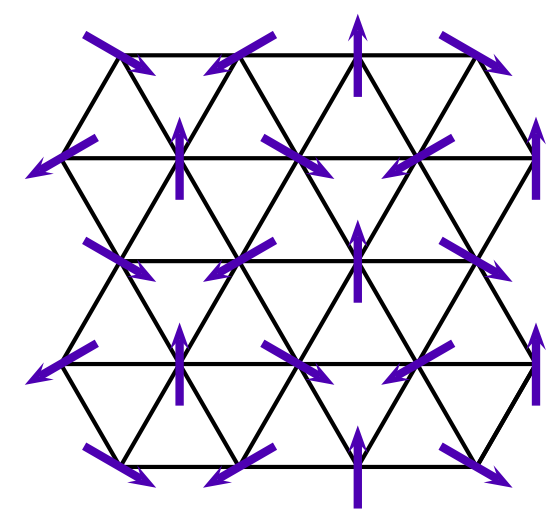

Figura 1.7: Orden tipo Néel en la red triangular antiferromagnética.

Las redes más estudiadas que no presentan orden antiferromagnético a nivel clásico y que podrían llegar a presentar desorden magnético son las llamadas corner-sharing-lattices, de las cuales las más conocidas son Kagomé, checkerboard y pyrochlore (figura 1.8). Estas redes están compuestas de unidades frustradas, triángulos y tetraedros, conectadas únicamente por los vértices. Esta baja conectividad detiene como consecuencia una alta degeneración para el estado fundamental clásico. Esto puede ser ilustrado en la red de Kagome en la figura 1.8a. Una plaqueta triangular con espines clásicos en los vértices, es mínimamente frustrada si los tres espines permanecen en el mismo plano formando un ángulo de $120^{\circ}$ entre ellos. El plano común puede ser especificado por un vector unitario $\mathbf{v}$ perpendicular a éste, cuya dirección (saliente o entrante al plano) queda definida por la orientación de los 


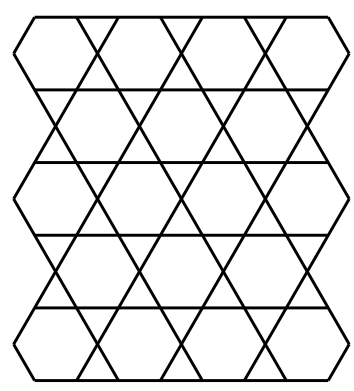

(a) Kagomé

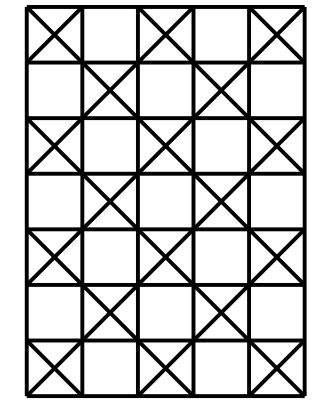

(b) Checkerboard

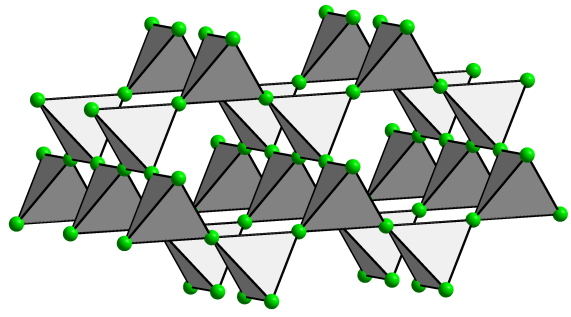

(c) Pyrochlore

Figura 1.8: Redes frustradas tipo corner-sharing.

espines en el triángulo (ver figura 1.9a). Entonces, una configuración de espines de mínima energía (formando un ángulo de $120^{\circ}$ entre ellos) puede ser identificada por un único espín y el vector unitario correspondiente $\mathbf{v}$. Dado que dos triángulos vecinos comparten únicamente un sitio, sus vectores unitarios son independientes: un plano asociado a un triángulo puede ser rotado alrededor del espín compartido sin costo de energía. La única restricción que se debe cumplir involucra a seis vectores v's de los triángulos que lo rodean a un hexágono como se muestra en la figura 1.9a: estos vectores determinan como transforma la orientación local de los espines al desplazarse de sitio en sitio alrededor del hexágono, y la transformación total al recorrer un círculo completo debe ser la identidad. Dado que el número de hexágonos es el doble del número de triángulos en una red de Kagomé, únicamente la mitad de los vectores v's quedan determinados. Consecuentemente, hay transformaciones continuas que transforman un estado fundamental clásico en otro, con lo que la degeneración clásica crece con el tamaño del sistema.

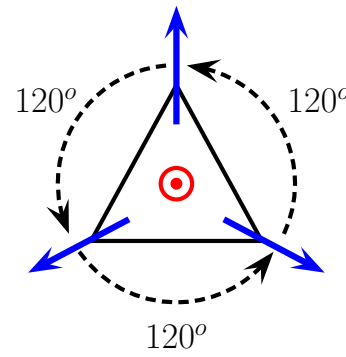

(a)

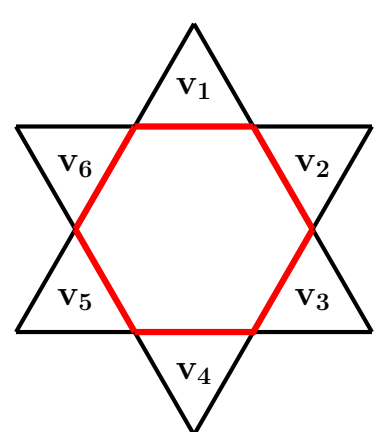

(b)

Figura 1.9: (a) El plano definido por los espines clásicos puede ser identificado por un vector perpendicular a éste, orientado acordando a la "regla de la mano derecha" con respecto a la dirección en que la orientación de los espines cambia en +120 . (b) Seis vectores de quiralidad $\mathbf{v}_{1} \ldots \mathbf{v}_{\mathbf{6}}$ alrededor de una plaqueta hexagonal 


\section{Interacciones competitivas}

Consideremos la otra situación donde un sistema de espines puede ser frustrado: este es el caso de diferentes clases de interacciones que entran en conflicto y de esta manera el estado fundamental no corresponde al mínimo de cada una de las interacciones. Por ejemplo, consideremos el hamiltoniano de Heisenberg unidimensional donde la interacción entre los espines a primeros vecinos $J_{1}$ es ferromagnética mientras que la interacción a segundos vecinos $J_{2}$ es antiferromagnética. Siempre que $J_{2}<\left|J_{1}\right| / 4$, el estado fundamental es ferromagnético: cada enlace $J_{1}$ es satisfecho energéticamente pero los enlaces $J_{2}$ no lo son. Por supuesto, cuando $J_{2}$ excede un valor crítico, el estado fundamental ferromagnético ya no es favorecido energéticamente y de esta manera, ambos enlaces $J_{1}$ y los $J_{2}$ no son completamente satisfechos.

Análogamente, existe una gran variedad de redes que presentan frustración debido a interacciones competitivas tanto cuasi-unidimensionales como bidimensionales (figura 1.10). Si bien

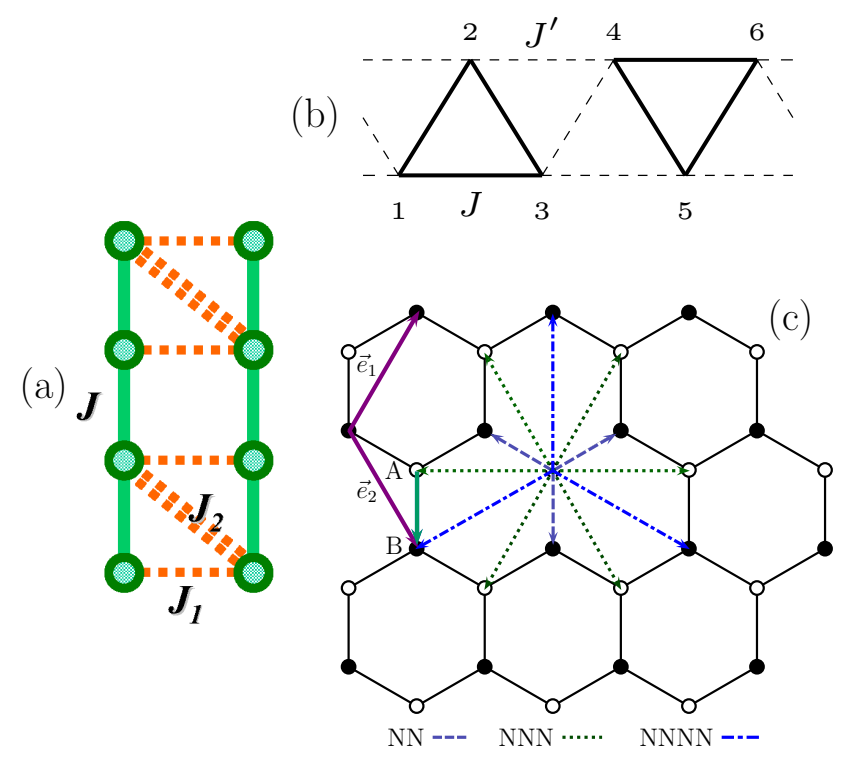

Figura 1.10: Redes frustradas geométricamente. (a) Escalera de espines frustrados por acoplamientos antiferromagnéticos $J, J_{1}$ y $J_{2}$. (b) Cadena de triángulos antiferromagnéticos $J>0$ acoplados con $J^{\prime}>0$, y (c) red hexagonal frustrada con acoplamientos antiferromagnéticos a primeros, segundos y terceros vecinos.

en la discusión anterior hemos hablado de frustración entre interacciones competitivas de la forma $J\left(\mathbf{S}_{i} \cdot \mathbf{S}_{j}\right)$, el concepto de frustración puede ser aplicado a otros tipos de interacciones tales como la interacción de Dzyaloshinski-Moriya $D\left(\mathbf{S}_{i} \wedge \mathbf{S}_{j}\right)$, interacción de plaquetas $k\left(\mathbf{S}_{i} \cdot \mathbf{S}_{j}\right)\left(\mathbf{S}_{k} \cdot \mathbf{S}_{l}\right), .$. etc.

Más adelante volveremos a tocar el tema de frustración cuando discutamos el problema de sistemas cuasi-unidimensionales de espines Heisenberg frustrados en el capítulo 4, y del modelo de Heisenberg frustrado en dos dimensiones en el capítulo 5. 


\subsection{Acoplamiento electrón-fonón}

Uno de los ingredientes que adicionaremos en esta tesis a los modelos unidimensionales de Hubbard y Heisenberg corresponde a los fonones adiabáticos. Como veremos en los capítulos 3 y 4, el acoplamiento de los grados de libertad de carga o espín con los fonones tiene consecuencias importantes sobre el sistema, modulando los ordenes de carga y magnetización respectivamente.

En esta sección, haremos un breve resumen sobre fonones e introduciremos el límite adiabático que es el que vamos a estudiar.

Consideremos el hamiltoniano unidimensional de $N$ átomos que describe las vibraciones armónicas de una red,

$$
\begin{aligned}
\mathcal{H}_{\text {fon'on }} & =\sum_{j}\left[\frac{p_{j}^{2}}{2 m}+\frac{m}{2} w_{o}^{2} \delta_{j}^{2}\right] \\
\delta_{j} & =u\left(r_{j+1}\right)-u\left(r_{j}\right), \quad \text { fonones acústicos } \\
\delta_{j} & =u\left(r_{j}\right), \text { fonones ópticos }
\end{aligned}
$$

donde $m$ es la masa de cada átomo y $w_{o}$ es la frecuencia de vibración. Aquí, $u_{j}$ representa los desplazamientos de los iones respecto de sus posiciones de equilibrio y $p_{j}$ son sus momentos conjugados.

Los fonones acústicos, corresponden a ondas de sonido en la red y la relación de dispersión no posee gap en su espectro (figura 1.11).

Por el contrario, los fonones ópticos, siempre tienen una frecuencia de vibración mínima

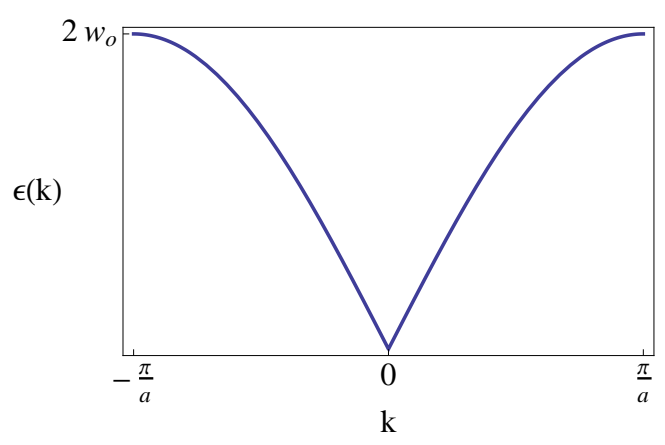

Figura 1.11:

incluso para longitudes de onda largas.

En el límite adiabático, se considera que los grados de libertad de los fonones "se congelan". Esto es, la frecuencia de vibración tiende a cero mientras que la masa de los átomos a infinito,

$$
\left\{w_{o} \rightarrow 0, m \rightarrow \infty\right\} \Rightarrow K \equiv m w_{o}^{2}=\text { constante }
$$

De esta manera, el hamiltoniano de los fonones queda únicamente descrito por las variables 
clásicas y estáticas $\delta_{j}$,

$$
\mathcal{H}_{\text {fonón }}=\frac{K}{2} \sum_{j} \delta_{j}^{2} \text {. }
$$

La interacción ya sea de los grados de libertad de espín (en el modelo de Heisenberg), como de carga (en el modelo de Hubbard) con los fonones es modelada por una expansión lineal de los acoplamientos de intercambio, $J_{i j}$ (Heisenberg) o de hopping, $t_{i j}$ (Hubbard) alrededor de los valores homogéneos, es decir

$$
\begin{aligned}
H_{\text {Heisenberg }} & =\sum_{i, j} J_{i j} \mathbf{S}_{i} \cdot \mathbf{S}_{j} \\
& \simeq \sum_{i, j} J\left(1+\alpha_{s-f}\left(u_{j}-u_{i}\right)\right) \mathbf{S}_{i} \cdot \mathbf{S}_{j} \\
& \Downarrow \\
H_{\text {espín-fonón }} & \sim \sum_{i, j} \alpha_{s-f}\left(u_{j}-u_{i}\right) \mathbf{S}_{i} \cdot \mathbf{S}_{j}
\end{aligned}
$$

donde $\alpha_{s-f}$ es una contante que mide la interacción espín-fonón. En el caso del modelo de Hubbard tenemos

$$
\begin{aligned}
H_{\text {Hubbard }} & =-\sum_{i, j, \sigma} t_{i j}\left(c_{i, \sigma}^{\dagger} c_{j, \sigma}+\text { h.c. }\right) \\
& \simeq-\sum_{i, j, \sigma} t\left(1+\alpha_{e-f}\left(u_{j}-u_{i}\right)\right)\left(c_{i, \sigma}^{\dagger} c_{j, \sigma}+\text { h.c. }\right) \\
& \Downarrow \\
H_{\text {electrón-fonón }} & \sim \sum_{i, j, \sigma} \alpha_{e-f}\left(u_{j}-u_{i}\right)\left(c_{i, \sigma}^{\dagger} c_{j, \sigma}+\text { h.c. }\right)
\end{aligned}
$$

donde $\alpha_{e-f}$ es una contante que mide la interacción electrón-fonón.

Peierls mostró en los 50's que en presencia de acoplamiento electrón-fonón y bajo ciertas condiciones, los sistemas de electrones unidimensionales presentan una inestabilidad hacia fases ailadoras [36]. En particular, a medio llenado, en un sistema de electrones acoplados a fonones, la red se dimeriza y se abre un gap en el espectro. Esta inestabilidad de conoce como inestabilidad de Peierls. De manera similar, el hamiltoniano de Heisenberg unidimensional acoplado con fonones, y a campo magnético externo cero, presenta una inestabilidad en la cual, los sitios se dimerizan y el orden antiferromagnético es reemplazado por un estado de singletes. Esta transición se conoce como transición de "espín-Peierls" [37]. La transición de espín-Peierls fue inicialmente estudiada en los años 70's, tanto teórica como experimentalmente. Sin embargo, la primera realización experimental fue observada en el compuesto orgánico $\mathrm{CuGeO}_{3}$ por Hase et. al en 1993 [38].

Además del acoplamiento de Peierls, existen materiales como los cristales moleculares (ver ejemplos en el capítulo 3), donde es importante otro tipo de interacción electrón-fonón 


\section{Acoplamiento electrón-fonón}

que se conoce como "acoplamiento de Holstein" [39]. Esta interacción está presente en los compuestos moleculares, y se debe a las vibraciones internas de las moléculas en cada sitio del cristal. Las vibraciones de las moleculas producen una desproporción local de la densidad de carga modificando la energía en el sitio. Este fenómeno puede tenerse en cuenta por medio del término de Holstein dado por,

$$
H_{\text {electrón-fonón }}^{\prime} \sim \sum_{i} \alpha \delta_{i} n_{i}
$$

donde $n_{i}$ es la densidad electrónica en el sitio $i, n_{i}=n_{i, \uparrow}+n_{i, \downarrow}$. 


\section{Capítulo 2}

\section{Métodos Analíticos y Numéricos}

En este capítulo presentaremos algunas de las técnicas más utilizadas para estudiar sistemas fuertemente correlacionados en bajas dimensiones. La primera parte será dedicada al el método conocido como Bosonización abeliana que es una de las herramientas más utilizadas para estudiar sistemas fermiónicos interactuantes unidimensionales. Existe mucha bibliografía sobre el tema y nosotros solo expondremos, en este capítulo y en el apéndice A, las ideas y resultados más importantes.

Posteriormente vamos a presentar dos transformaciones que permiten tratar sistemas de espines en términos de variables bosónicas: la primera conocida como transformación de Holstein-Primakoff y la segunda transformación de Schwinger. Estas técnicas tienen la ventaja de que permiten estudiar sistemas más allá de $d=1$.

\subsection{Bosonización Abeliana, $d=1$.}

La idea básica de bosonización se basa en el hecho de que las excitaciones hueco-partícula de baja energía son de carácter bosónico (ver sección 1.2.3), y de esta manera el espectro del gas de electrones puede se estudiado en término de estas excitaciones. La cuestión fue planteada por F. Bloch en 1934, pero Tomonaga mostró en 1950 que esto puede ser verdad solo en una dimensión (espacial). La razón es simple: Consideremos la figura 1.3b. En la parte izquierda se ilustra la creación de un par partícula-hueco de momento $k$. En la derecha, se muestra el espectro de las excitaciones partícula-hueco creadas por encima del nivel de Fermi (ambas cantidades medidas con respecto al estado fundamental). Debido a la dispersión lineal cerca del nivel de Fermi, los pares tienen dispersión tipo-cuasi-partícula cerca de momento cero, así estas pueden propagarse de manera coherente. En otras palabras, la partícula y el hueco tienen aproximadamente la misma velocidad de grupo a bajas energías y se propagan juntas. Cualquier atracción por débil que sea, tiene una gran consecuencia, es decir, liga al par a una propagación coherente: "una nueva partícula". En particular, en el límite de bajas energías, las excitaciones partícula-hueco en una dimensión son exactamente coherentes.

En la figura 1.3a se muestra la situación correspondiente en dos dimensiones, con una superficie de Fermi circular. Está claro que un par partícula-hueco con un dado momento $k$ puede tener un espectro continuo de energías comenzando en cero. Esto se debe a que 
40 Bosonización Abeliana, $d=1$.

uno puede aniquilar una partícula en un punto por debajo del nivel de Fermi y crearla en cualquier punto desde 0 a $2 k_{F}$. Entonces, al ser continuo el espectro partícula-hueco, las interacciones tienen una dura tarea en formar coherentemente pares partícula-hueco. Esto implica que definir la teoría en términos de variables bosónicas es mucho menos obvio en dimensiones mayores que uno.

La pregunta que surge es: ¿existe algún operador asociado al par en término de operadores de los electrones?

\subsubsection{Fermiones libres}

Para responder a la pregunta antes planteada, comenzamos con un gas de electrones libres sin espín sobre una red unidimensional de $N$ sitios, descrita por un hamiltoniano

$$
\mathcal{H}_{t-\mu}=-t \sum_{j=1}^{N}\left(c_{j+1}^{\dagger} \cdot c_{j}+h . c .\right)+\mu \sum_{j} n_{j}
$$

La trasformada de Fourier del hamiltoniano libre está dada por:

$$
\begin{aligned}
\mathcal{H}_{t-\mu} & =\int_{k \in P Z B} \frac{d k}{2 \pi} \varepsilon(k) c^{\dagger}(k) \cdot c(k) \\
\varepsilon(k) & =-2 t \cos (k a)+\mu \\
c(k) & =\frac{1}{\sqrt{N}} \sum_{j=1}^{N} e^{i a j k} c_{j} \\
\left\{c_{k}, c_{k^{\prime}}^{\dagger}\right\} & =2 \pi \delta\left(k-k^{\prime}\right)
\end{aligned}
$$

donde $c^{\dagger}(k)$ (respectivamente $c(k)$ ) crea (aniquila) un fermión con vector de onda $k$. Como estamos interesados en el límite de baja energía del modelo (2.1), que es donde las excitaciones partícula-hueco son coherentes, definimos operadores de creación y aniquilación $\alpha(k)$ y $\beta(k)$, en la vecindad de los puntos de Fermi $\pm k_{F}$ (ver figura 2.1) como sigue:

$$
\begin{aligned}
\alpha(k) & =c\left(k_{F}+k\right) \\
\alpha(-k) & =c\left(-k_{F}+k\right) \\
\beta(k) & =c^{\dagger}\left(k_{F}-k\right) \\
\beta(-k) & =c^{\dagger}\left(-k_{F}+k\right)
\end{aligned}
$$

donde $k>0$. Luego restringimos la integración alrededor de los puntos de Fermi adicionando un cutoff ultravioleta $\Lambda$ que depende de la constante de red $a\left(\Lambda \ll k_{F}\right)$ tal que $|k| \leq \Lambda$.

El hamiltoniano no interactuante 2.2 toma la forma:

$$
\mathcal{H}_{t-\mu}=\int \frac{d k}{2 \pi} v_{F}|k|\left[\alpha^{\dagger}(k) \cdot \alpha(k)+\beta^{\dagger}(k) \cdot \beta(k)\right]
$$




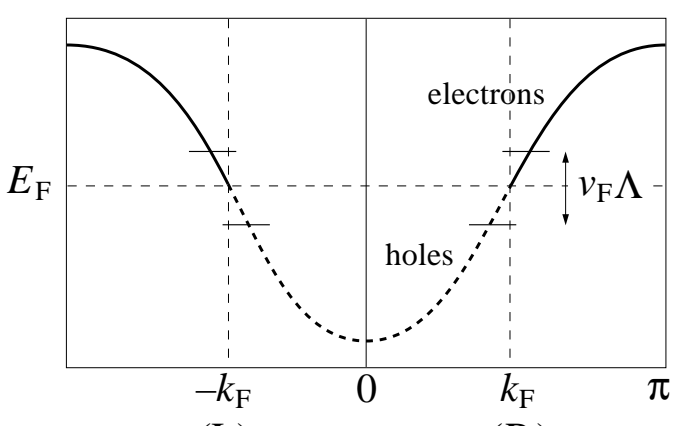

(L) $\quad(\mathrm{R})$

Figura 2.1: Relación de dispersión para un gas de electrones en $d=1$.

donde hemos introducido la velocidad de Fermi $v_{F}$, que no es otra cosa que la pendiente de la aproximación lineal en $\pm k_{F}$ y es el único parámetro remanente de la teoría microscópica,

$$
\left.\frac{d \varepsilon(k)}{d k}\right|_{k=k_{F}}=2 t a \sin \left(k_{f} a\right)=v_{f} .
$$

La energía es ahora definida con respecto al estado fundamental y la integración se realiza entre $-\Lambda$ y $\Lambda$. En el espacio de coordenadas, el desarrollo alrededor de los puntos de Fermi permite introducir campos suaves $\psi_{R}(x)$ y $\psi_{L}(x)$ denominados quirales, correspondientes a los puntos $+k_{F} \mathrm{y}-k_{F}$ respectivamente,

$$
\begin{aligned}
c(x) & \equiv \frac{c_{j}}{\sqrt{a}} \\
& \equiv \psi_{R}(x) e^{i k_{F} x}+\psi_{L}(x) e^{-i k_{F} x}+\ldots
\end{aligned}
$$

donde $x=j a \mathrm{y} \ldots$ indican correcciones de orden superior cerca de los puntos de Fermi. El desarrollo de los campos $\psi_{R, L}(x)$ en términos de los operadores $\alpha^{\prime} s$ y $\beta^{\prime} s$ es

$$
\begin{aligned}
\psi_{R}(x) & =\int_{k>0} \frac{d k}{2 \pi}\left[e^{i k x} \alpha(k)+e^{-i k x} \beta^{\dagger}(k)\right] \\
\psi_{L}(x) & =\int_{k<0} \frac{d k}{2 \pi}\left[e^{i k x} \alpha(k)+e^{-i k x} \beta^{\dagger}(k)\right]
\end{aligned}
$$

El factor $\sqrt{a}$ por un lado, se introduce para dar la correcta relación de anticonmutación tipo delta, y por otro refleja su dimensión:

$$
\begin{aligned}
& \left\{\psi_{R}(x), \psi_{R}^{\dagger}\left(x^{\prime}\right)\right\}=\delta\left(x-x^{\prime}\right) \\
& \left\{\psi_{L}(x), \psi_{L}^{\dagger}\left(x^{\prime}\right)\right\}=\delta\left(x-x^{\prime}\right) \\
& \left\{\psi_{R}(x), \psi_{L}^{\dagger}\left(x^{\prime}\right)\right\}=0
\end{aligned}
$$

En el límite continuo, podemos reescribir el hamiltoniano no interactuante (2.2) en términos de campos fermiónicos como:

$$
\mathcal{H}_{t-\mu}=i v_{F} \int d x\left[\psi_{R}^{\dagger}(x) \partial_{x} \psi_{R}(x)-\psi_{L}^{\dagger}(x) \partial_{x} \psi_{L}(x)\right]
$$


Bosonización Abeliana, $d=1$.

Hay que aclarar que la ecuación (2.11) es engañosa: puede hacer pensar que los modos positivos son continuos hacia los modos negativos, sin embargo este no es el caso debido al cut off en las integrales y por lo tanto ambos sectores están separados en $2 k_{F}$.

A partir de estos campos se definen los operadores densidad

$$
J_{R}(x)=\psi_{R}^{\dagger}(x) \psi_{R}(x), \quad J_{L}(x)=\psi_{L}^{\dagger}(x) \psi_{L}(x) .
$$

Para la descripción de electrones, es necesario considerar fermiones con espín $\sigma=\uparrow, \downarrow$. En este caso, el procedimiento es exactamente igual que el antes descrito. El operador fermionico cerca del nivel de Fermi $\left( \pm k_{F, \uparrow}\right.$ para el sector $\uparrow$ y $\pm k_{F, \downarrow}$ para el $\left.\downarrow\right)$ está dado porque

$$
\begin{aligned}
c_{\sigma}(x) & \equiv \frac{c_{j, \sigma}}{\sqrt{a}} \\
& \equiv \psi_{R, \sigma}(x) e^{i k_{F, \sigma} x}+\psi_{L, \sigma}(x) e^{-i k_{F, \sigma} x}+\ldots
\end{aligned}
$$

donde los puntos indican términos de orden superior. Por lo general, estas correcciones no son incluidas en el estudio de baja energía de modelos como el de Hubbard, Heisenberg, $t-J$, debido a que solo aportan términos menos relevantes ${ }^{1} \mathrm{y}$ por lo tanto no modifican el análisis. Sin embargo, como veremos en el capítulo 3, éstas correcciones juegan un papel muy importante en la descripción de baja energía del modelo de Hubbard acoplado a fonones adiabáticos.

El resultado final consiste de dos copias desacopladas con distinto índice de espín:

$$
\mathcal{H}_{t-\mu}=i \sum_{\sigma=\uparrow, \downarrow} \int d x v_{F, \sigma}\left[\psi_{R, \sigma}^{\dagger}(x) \partial_{x} \psi_{R, \sigma}(x)-\psi_{L, \sigma}^{\dagger}(x) \partial_{x} \psi_{L, \sigma}(x)\right]
$$

donde la velocidad de Fermi y los vectores de Fermi satisfacen $v_{F, \uparrow}=v_{F, \downarrow}=v_{F}$ y $k_{F, \uparrow}=$ $k_{F, \downarrow}=k_{F}$ en el caso de campo magnético externo $h=0$ (ver apéndice A).

\subsubsection{Fermiones interactuantes}

La interacción más general de dos cuerpos entre electrones en una dimensión (de una banda únicamente) es

$$
\mathcal{H}_{\text {int }}=\frac{1}{2} \sum_{\sigma, \sigma^{\prime}} \sum_{k_{1}, k_{2}, k_{3}, k_{4}} V_{\sigma, \sigma^{\prime}} c\left(k_{1}, k_{2}, k_{3}, k_{4}\right) c_{\sigma}^{\dagger}\left(k_{1}\right) c_{\sigma^{\prime}}^{\dagger}\left(k_{2}\right) c_{\sigma^{\prime}}\left(k_{3}\right) c_{\sigma}\left(k_{4}\right)
$$

En el límite de baja energía, los procesos de dispersión están naturalmente restringidos a la vecindad de los puntos de Fermi, y se restringen a únicamente cuatro tipos, ilustrados en la

\footnotetext{
${ }^{1}$ En el apéndice A discutimos la idea de operadores relevantes e irrelevantes.
} 
figura 2.2. En términos de campos continuos, las correspondientes densidades hamiltonianas son:

$$
\begin{aligned}
h_{1} & =v_{f} g_{1} \sum_{\sigma} \psi_{R, \sigma}^{\dagger}(x) \psi_{L, \sigma}(x) \psi_{L,-\sigma}^{\dagger}(x) \psi_{R,-\sigma}(x) \\
h_{2}^{c} & =v_{f} g_{2, c}\left(J_{R, \uparrow}(x)+J_{R, \downarrow}(x)\right)\left(J_{L, \uparrow}(x)+J_{L, \downarrow}(x)\right) \\
h_{2}^{s} & =v_{f} g_{2, s}\left(J_{R, \uparrow}(x)-J_{R, \downarrow}(x)\right)\left(J_{L, \uparrow}(x)-J_{L, \downarrow}(x)\right) \\
h_{3} & =\frac{1}{2} v_{f} g_{3} \sum_{\sigma} \psi_{R, \sigma}^{\dagger}(x) \psi_{R,-\sigma}^{\dagger}(x) \psi_{L, \sigma}(x) \psi_{L,-\sigma}(x)+h . c . \\
h_{4}^{c} & =\frac{1}{2} v_{f} g_{4, c}\left[\left(J_{R, \uparrow}(x)+J_{R, \downarrow}(x)\right)^{2}+\left(J_{L, \uparrow}(x)+J_{L, \downarrow}(x)\right)^{2}\right] \\
h_{4}^{s} & =\frac{1}{2} v_{f} g_{4, s}\left[\left(J_{R, \uparrow}(x)-J_{R, \downarrow}(x)\right)^{2}+\left(J_{L, \uparrow}(x)-J_{L, \downarrow}(x)\right)^{2}\right]
\end{aligned}
$$
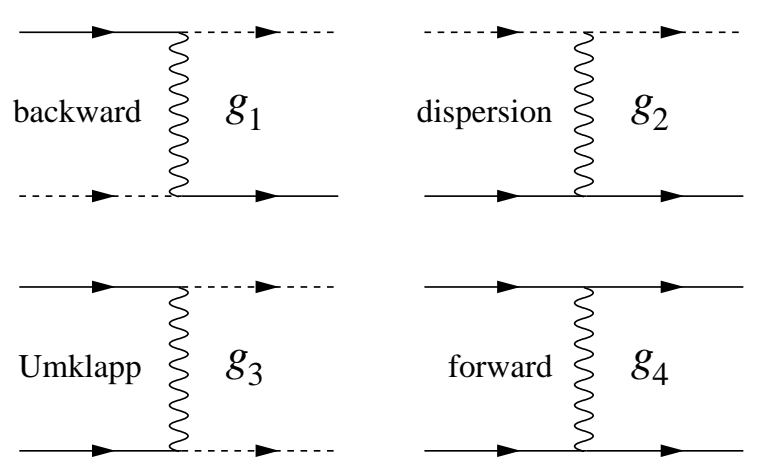

Figura 2.2: Los cuatro procesos posibles para electrones en una dimensión moviéndose desde la derecha (línea continua) y desde la izquierda (línea a trazos).

- El proceso $g_{1}$ se denomina backscattering y acopla fermiones de un punto de la superficie de Fermi con el otro. Los fermiones cambian de rama después de la interacción.

- $g_{2}$ (dispersión), al igual que $g_{1}$ acopla fermiones $R$ y $L$. Sin embargo, luego de la interacción estos permanecen del mismo lado.

- $g_{3}$ corresponde al proceso umklapp y es permitido únicamente a llenado $n=1$.

- $g_{4}$ corresponde a forward scattering y acopla fermiones del mismo lado.

Veamos ahora que procesos aparecen por la interacción coulombiana en el sitio:

$$
\mathcal{H}_{U}=U \sum_{j} n_{j, \uparrow} \cdot n_{j, \downarrow}
$$


Usando las expresiones (2.10) y (2.14) el límite continuo de este término es

$$
\begin{aligned}
\mathcal{H}_{U}= & a^{2} U \int d x\left(J_{R, \uparrow}+J_{L, \uparrow}\right)\left(J_{R, \downarrow}+J_{L, \downarrow}\right) \\
& +a^{2} U \int d x\left(e^{i 4 k_{F} x} \psi_{R, \uparrow}^{\dagger}(x) \psi_{L, \uparrow}(x) \psi_{R, \downarrow}^{\dagger}(x) \psi_{L, \downarrow}(x)+\text { h.c. }\right) \\
& +a^{2} U \int d x\left(\psi_{R, \uparrow}^{\dagger}(x) \psi_{L, \uparrow}(x) \psi_{L, \downarrow}^{\dagger}(x) \psi_{R, \downarrow}(x)+\text { h.c. }\right) .
\end{aligned}
$$

El término que contiene $e^{i 4 k_{F} x}$ corresponde al proceso umklapp y es inconmensurado en el sentido que integra a cero, a menos que $k_{F}=\pi / 2$, que corresponde a medio llenado $n=1^{2}$.

\subsubsection{Reglas de bosonización}

En esta sección presentaremos las reglas de Bosonización [18, 30, 40, 41, 42] y dejamos para el apéndice A la muchos de los detalles técnicos.

La equivalencia entre excitaciones de pares hueco-partícula y excitaciones bosónicas, se formaliza mediante un mapeo de los fermiones quirales $\psi_{R, \sigma}$ y $\psi_{L, \sigma}$ a campos bosónicos quirales $\phi_{R, \sigma} \mathrm{y} \phi_{L, \sigma}$,

$$
\begin{aligned}
& \psi_{R, \sigma}=\eta_{R, \sigma} \frac{1}{\sqrt{2 \pi a}}: e^{i \sqrt{4 \pi} \phi_{R, \sigma}}: \\
& \psi_{L, \sigma}=\eta_{L, \sigma} \frac{1}{\sqrt{2 \pi a}}: e^{-i \sqrt{4 \pi} \phi_{L, \sigma}}:
\end{aligned}
$$

donde los factores $\eta_{m, \sigma}(m=R, L)$ son Hermíticos y se conocen como factores de Klein; estos factores obedecen el álgebra de Clifford

$$
\left\{\eta_{\mu}, \eta_{\nu}\right\}=\left\{\bar{\eta}_{\mu}, \bar{\eta}_{\nu}\right\}=2 \delta_{\mu \nu} \quad\left\{\eta_{\mu}, \bar{\eta}_{\nu}\right\}=0, \quad \mu, \nu=(R, \uparrow ; L, \uparrow ; R, \downarrow ; L, \downarrow)
$$

donde y se introducen para que las distintas especies fermiónicas anticonmuten. El espacio de Hilbert sobre el que estos actúan, tiene una mínima dimensión que depende del número de especies fermiónicas. Con cuatro especies $(R \uparrow, L \uparrow, R \downarrow, L \downarrow)$, la dimensión mínima es cuatro, al igual que las matrices de Dirac un espacio-tiempo de $3+1$ dimensiones. Dado que los factores de Klein actúan sobre un espacio de Hilbert que difiere del espacio de los bosones quirales, el producto de factores puede ser diagonalizado y reemplazado por alguno de sus autovalores.

La notación : : indica orden normal de operadores mientras que los operadores $e^{i \alpha \phi}$ se conocen como operadores de vértice [29]. Los productos de operadores fermiónicos se vuelven, después de la bosonización, en producto de operadores de vértice. Si los campos bosónicos

\footnotetext{
${ }^{2} \mathrm{~A}$ campo magnético externo $h=0, k_{F, \uparrow}=k_{F, \downarrow}=n \pi / 2$, donde el llenado está dado por $n=N_{e} / N$.
} 
tienen diferentes grados de libertad de $(R, L$ y $\sigma)$ estos productos son simplemente exponenciales de la suma. De otra manera, debido al orden normal, se tiene que tener en cuenta la función de Green de la teoría bosónica. El resultado final queda (ver detalles en apéndice A):

$$
: e^{i \alpha \phi_{r, \sigma}(z)}:: e^{i \beta \phi_{r, \sigma}(w)}:=|z-w|^{\frac{\alpha \beta}{4 \pi}}: e^{i \alpha \phi_{r, \sigma}(z)+i \beta \phi_{r, \sigma}(w)}:
$$

$\operatorname{con}^{3} r=R, L, z=-i\left(x-v_{F} t\right)$ y $w=-i\left(y-v_{F} t\right)$.

Además, el orden normal de productos definidos en el mismo punto es calculado mediante:

$$
: \psi_{r, \sigma}^{\dagger}(z) \psi_{r, \sigma}(z):=\lim _{\varepsilon \rightarrow \infty}\left[\psi_{r, \sigma}^{\dagger}(z+\varepsilon) \psi_{r, \sigma}(z)-\left\langle\psi_{r, \sigma}^{\dagger}(z+\varepsilon) \psi_{r, \sigma}(z)\right\rangle\right]
$$

Usando la expresión anterior, se puede mostrar que las corrientes definidas en (2.14) quedan expresadas como:

$$
\begin{aligned}
J_{R}(z) & =-\frac{i}{\sqrt{\pi}} \partial_{z} \phi_{R, \sigma}(z) \\
J_{L}(\bar{z}) & =\frac{i}{\sqrt{\pi}} \partial_{\bar{z}} \phi_{L, \sigma}(\bar{z})
\end{aligned}
$$

A partir de los campos $\phi_{R, \sigma}$ y $\phi_{L, \sigma}$, se introducen otros dos que serán de gran importancia posteriormente: el campo $\varphi$ y su dual $\theta$ como:

$$
\begin{aligned}
\varphi_{\sigma}(x) & =\phi_{R, \sigma}(x)+\phi_{L, \sigma}(x) \\
\theta_{\sigma}(x) & =\phi_{R, \sigma}(x)+\phi_{L, \sigma}(x) .
\end{aligned}
$$

El campo $\theta_{\sigma}(x)$ se conoce como bosón dual y su derivada espacial define el momento canónico de $\varphi_{\sigma}(x)$ a través de la relación $\partial_{x} \theta_{\sigma}(x)=-\frac{1}{v} \partial_{t} \varphi(x)$.

En la sección siguiente utilizaremos las reglas de bosonización presentadas en esta sección y las aplicaremos a dos modelos que utilizamos en los capítulos 3 y 4: el modelo de Hubbard y el modelo de Heisenberg.

\subsubsection{Bosonización del Modelo de Hubbard y Heisenberg}

En esta sección vamos a presentar las versiones bosonizadas del modelo de Hubbard y de Heisenberg unidimensional.

\footnotetext{
${ }^{3}$ En el apéndice $\mathrm{A}$ introducimos las coordenadas complejas $z=-i\left(x-v_{F} t\right)$ y $\bar{z}=i\left(x+v_{F} t\right)$, muy convenientes para realizar los cálculos.
} 
46 Bosonización Abeliana, $d=1$.

\section{Modelo de Hubbard}

Usando las reglas de bosonización, la parte no interactuante del hamiltoniano de Hubbard en el sector de baja energía definido en la ecuación (2.16) queda:

$$
\begin{aligned}
\mathcal{H}_{t-\mu} & =i v_{F} \sum_{\sigma=\uparrow, \downarrow} \int d x\left[\psi_{R, \sigma}^{\dagger}(x) \partial_{x} \psi_{R, \sigma}(x)-\psi_{L, \sigma}^{\dagger}(x) \partial_{x} \psi_{L, \sigma}(x)\right] \\
& =v_{F} \sum_{\sigma=\uparrow, \downarrow} \int d x\left[\left(\partial_{x} \phi_{R, \sigma}(x)\right)^{2}+\left(\partial_{x} \phi_{L, \sigma}(x)\right)^{2}\right] \\
& =\frac{v_{F}}{2} \sum_{\sigma=\uparrow, \downarrow} \int d x\left[\left(\partial_{x} \varphi_{\sigma}(x)\right)^{2}+\left(\partial_{x} \theta_{\sigma}(x)\right)^{2}\right] .
\end{aligned}
$$

La expresión anterior muestra que a bajas energías, el hamiltoniano fermiónico no interactuante equivale al de bosones libres con velocidad $v_{F}$.

La parte de interacción (2.20) es bosonizada como:

$$
\begin{aligned}
\mathcal{H}_{U}= & \frac{a U}{\pi} \int d x \partial_{x} \varphi_{\uparrow} \partial_{x} \varphi_{\downarrow}+\frac{U}{2 \pi^{2} a} \int d x: \cos \sqrt{4 \pi}\left(\varphi_{\uparrow}-\varphi_{\downarrow}\right): \\
& +\frac{U}{2 \pi^{2} a} \int d x: \cos \sqrt{4 \pi}\left(\varphi_{\uparrow}+\varphi_{\downarrow}\right):
\end{aligned}
$$

recordemos que el último término corresponde al proceso Umklapp, permitido únicamente a medio llenado (es decir un electrón por sitio).

El punto interesante aquí es que, en los cosenos, la dependencia en los campos es $\left(\varphi_{\uparrow}-\varphi_{\downarrow}\right)$ y $\left(\varphi_{\uparrow}+\varphi_{\downarrow}\right)$ respectivamente. De esta manera, podemos definir nuevos campos denominados de "carga" y "espín" como

$$
\varphi_{c}=\frac{\varphi_{\uparrow}+\varphi_{\downarrow}}{\sqrt{2}}, \quad \varphi_{s}=\frac{\varphi_{\uparrow}-\varphi_{\downarrow}}{\sqrt{2}}
$$

Usando estos nuevos campos, el hamiltoniano es completamente separable y podemos escribir $\mathcal{H}_{\text {Hubbard }}=\mathcal{H}_{t-\mu}+\mathcal{H}_{U}=\mathcal{H}_{c}+\mathcal{H}_{s}$ como

$$
\begin{aligned}
& \mathcal{H}_{c}=\int d x \frac{v_{F}}{2}\left[\left(\partial_{x} \theta_{c}\right)^{2}+\left(1+\frac{U}{\pi v_{F}}\right)\left(\partial_{x} \varphi_{c}\right)^{2}\right]+\frac{U}{2 \pi^{2} a} \cos \sqrt{8 \pi} \varphi_{c} \\
& \mathcal{H}_{s}=\int d x \frac{v_{F}}{2}\left[\left(\partial_{x} \theta_{s}\right)^{2}+\left(1-\frac{U}{\pi v_{F}}\right)\left(\partial_{x} \varphi_{s}\right)^{2}\right]+\frac{U}{2 \pi^{2} a} \cos \sqrt{8 \pi} \varphi_{s}
\end{aligned}
$$

Definiendo unos nuevos parámetros, el hamiltoniano se puede escribir como:

$$
\begin{aligned}
\mathcal{H}_{c} & =\int d x \frac{v_{c}}{2}\left[K_{c}\left(\partial_{x} \theta_{c}\right)^{2}+\frac{1}{K_{c}}\left(\partial_{x} \varphi_{c}\right)^{2}\right]+g_{c} \cos \sqrt{8 \pi} \varphi_{c} \\
\mathcal{H}_{s} & =\int d x \frac{v_{s}}{2}\left[K_{s}\left(\partial_{x} \theta_{s}\right)^{2}+\frac{1}{K_{s}}\left(\partial_{x} \varphi_{s}\right)^{2}\right]+g_{s} \cos \sqrt{8 \pi} \varphi_{s}
\end{aligned}
$$


donde

$$
\begin{array}{ll}
K_{c}=\left(1+\frac{a U}{v_{F} \pi}\right)^{-1 / 2} & v_{c}=v_{F} \sqrt{1-\frac{a U}{v_{F} \pi}} \quad g_{c}=\frac{U}{2 \pi^{2} a} \\
K_{s}=\left(1-\frac{a U}{v_{F} \pi}\right)^{-1 / 2} & v_{s}=v_{F} \sqrt{1+\frac{a U}{v_{F} \pi}} \quad g_{s}=\frac{U}{2 \pi^{2} a}
\end{array}
$$

donde $K_{c, s}$ y $v_{c, s}$ son el parámetro de Luttinger y la velocidad para el sector de carga y espín. El primer término corresponde a un líquido de Luttinger con parámetros de Luttinger $K_{c, s} \mathrm{y}$ velocidad $v_{c, s}$, mientras que el coseno corresponde al término de autointeracción. Reescritos de esta manera, tanto el sector de carga como el de espín quedan descriptos por el modelo conocido como seno-Gordon, una de las teorías de campos unidimensionales más estudiadas [43].

Dependiendo de los valores de $K_{c, s}$, el coseno puede abrir un gap de carga o espín, si éste es un operador relevante en el sentido del grupo de renormalización. Para saber esto, es necesario calcular la dimensión conforme $\Delta$ definida en el apéndice A; este será relevante si $\Delta<2$, marginal si $\Delta=2$ e irrelevante si $\Delta>2$.

En nuestro caso tenemos, con $U>0$ (ver detalles en el apéndice A):

$$
\begin{aligned}
\Delta_{c} & =\frac{2}{1+\frac{a U}{\pi v_{F}}}<2 \\
\Delta_{s} & =\frac{2}{1+\frac{a U}{\pi v_{F}}}>2
\end{aligned}
$$

indicando que el sector de carga el sistema posee un gap mientras que en el sector de espín corresponde a un líquido de Luttinger con parámetros $v_{s}$ y $K_{s}$.

\section{Modelo de Heisenberg}

El siguiente modelo que vamos a estudiar mediante bosonización es el modelo de Heisenberg unidimensional dado por el hamiltoniano

$$
\mathcal{H}_{J-J_{z}}=\sum_{i=1}^{N}\left[\frac{J}{2}\left(S_{i}^{+} S_{i+1}^{-}+S_{i+1}^{+} S_{i}^{-}\right)+J_{z} S_{i}^{z} \cdot S_{i+1}^{z}\right]
$$

donde las interacciones son solo entre primeros vecinos, y $J>0$. Este modelo tiene solución exacta mediante el ansatz de Bethe, revelando que el modelo no tiene gap en sus excitaciones para $-J \leq J_{z} \leq J$, y existe una transición de fase hacia un fase con $\operatorname{gap}\left(J_{z}>J\right)$ en el punto isotrópico $J_{z}=J$.

Para los modelos de espín $S=1 / 2$ en $d=1$, existe una transformación que permite mapear los operadores de espín a operadores fermiónicos. Esta transformación se conoce como transformación de Jordan-Wigner [44] y se basa en el hecho de que cada espín $S=1 / 2$ tiene dos 
estados en cada sitio, $\uparrow \mathrm{y} \downarrow$. El mapeo identifica estos estados, en términos de los estados de fermiones en donde un espín $\downarrow$ corresponde a un sitio desocupado y un espín $\uparrow$ a un sitio ocupado. Para implementar esto, se define un operador fermiónico de $f_{i}$ en cada sitio y se construye la siguiente representación para los operadores de espín:

$$
\begin{aligned}
S_{i}^{z} & =f_{i}^{\dagger} f_{i}-1 / 2=n_{i}-1 / 2 \\
S_{i}^{-} & =(-1)^{i} f_{i} e^{i \pi \sum_{j=1}^{i-1} n_{j}}
\end{aligned}
$$

El factor exponencial de la suma de operadores, llamado operador de string, tiene que incluirse para que, mientras fermiones en distintos sitios anticonmutan, espines en distintos sitios conmuten. Uno puede chequear, que efectivamente se satisfacen las relaciones de conmutación de $\mathrm{SU}(2) y$ anticonmutación

$$
\begin{aligned}
{\left[S_{i}^{ \pm}, S_{j}^{z}\right] } & = \pm \frac{1}{2} S_{j}^{ \pm} \delta_{i, j} \\
\left\{f_{i}, f_{j}^{\dagger}\right\} & =\delta_{i, j} \\
\left\{f_{i}, f_{j}\right\} & =0
\end{aligned}
$$

Ahora reescribimos el hamiltoniano (2.40) en términos de fermiones, obteniendo

$$
\begin{aligned}
\mathcal{H}_{J-J_{z}} & =-\frac{J}{2} \sum_{j}\left[f_{j}^{\dagger} e^{i \pi n_{j}} f_{j+1}+\text { h.c. }\right]+J_{z} \sum_{j}\left[\left(n_{j}-1 / 2\right)\left(n_{j+1}-1 / 2\right)\right] \\
& =-\frac{J}{2} \sum_{j}\left[f_{j}^{\dagger} f_{j+1}+\text { h.c. }\right]+J_{z} \sum_{j}\left[\left(n_{j}-1 / 2\right)\left(n_{j+1}-1 / 2\right)\right]
\end{aligned}
$$

Notemos que el término $\left(S_{i}^{+} S_{i+1}^{-}+\right.$h.c. $)$del hamiltoniano de espines, reescrito en términos de los operadores fermiónicos $f$ 's, es análogo al hopping en el hamiltoniano electrónico y corresponde a la parte cinética; mientras que la interacción $S^{z}-S^{z}$, que equivale a una interacción cuártica en fermiones, es análoga al término de repulsión coulombiana.

Utilizando las reglas de bosonización (2.10) y (2.22), la parte cinética del hamiltoniano (2.43) queda

$$
\begin{aligned}
\mathcal{H}_{J} & =-i v_{F} \int d x\left[\psi_{R}^{\dagger} \partial_{x} \psi_{R}-\psi_{L}^{\dagger} \partial_{x} \psi_{L}\right] \\
& =v_{F} \int d x\left[\left(\partial_{x} \phi_{R}(x)\right)^{2}+\left(\partial_{x} \phi_{L}(x)\right)^{2}\right] \\
& =\frac{v_{F}}{2} \int d x\left[\left(\partial_{x} \varphi(x)\right)^{2}+\left(\partial_{x} \theta(x)\right)^{2}\right]
\end{aligned}
$$

donde $v_{F}=2 a J \sin \left(k_{F} a\right), k_{F}=\pi / 2$ a campo magnético externo cero.

El término $J_{z}$ se incluye como una perturvación del hamiltoniano libre y en el lenguaje fermiónico se escribe

$$
\mathcal{H}_{J_{z}}=J_{z} \sum_{j}: f_{j}^{\dagger} f_{j}:: f_{j+1}^{\dagger} f_{j+1}:
$$


En el límite de baja energía, reescribimos los operadores fermiónicos usando (2.10) como

$$
\mathcal{H}_{J_{z}}=a^{2} J_{z} \int d x\left[\rho(x)+(-1)^{x / a} D(x)\right] \cdot\left[\rho(x+a)+(-1)^{1+x / a} D(x+a)\right]
$$

donde $\rho(x)=: \psi_{R}^{\dagger} \psi_{R}:+\psi_{L}^{\dagger} \psi_{L}:$ y $D(x)=\psi_{L}^{\dagger} \psi_{R}+\psi_{R}^{\dagger} \psi_{L}$.

Expandiendo a primer orden en $a$ y eliminando términos oscilatorios obtenemos:

$$
\mathcal{H}_{J_{z}}=a^{2} J_{z} \int d x\left[4 J_{R}(x) J_{L}(x)+J_{R}^{2}(x)+J_{L}^{2}(x)-\left(\left(\psi_{L}^{\dagger}(x) \psi_{R}(x)\right)^{2}+\text { h.c. }\right)\right] .
$$

Usando (2.22) obtenemos

$$
\begin{aligned}
\mathcal{H}_{J_{z}}= & a^{2} J_{z} \int d x \frac{1}{\pi}\left[4 \partial_{x} \phi_{L} \partial_{x} \phi_{R}-\left(\partial_{x} \phi_{R}\right)^{2}-\left(\partial_{x} \phi_{L}\right)^{2}\right] \\
& +\frac{J_{z}}{2 \pi^{2}} \int d x \cos \left(2 \sqrt{4 \pi}\left(\phi_{R}+\phi_{L}\right)\right)
\end{aligned}
$$

finalmente el hamiltoniano $H_{J-J_{z}}$ queda

$$
\begin{aligned}
\mathcal{H}_{J-J_{z}} & =\int d x \frac{v}{2}\left[K\left(\partial_{x} \theta\right)^{2}+\frac{1}{K}\left(\partial_{x} \varphi\right)^{2}\right]+g \cos 2 \sqrt{4 \pi} \varphi \\
K & =\left(\frac{1-\frac{3 a J_{z}}{\pi v_{F}}}{1+\frac{a J_{z}}{\pi v_{F}}}\right)^{1 / 2} \\
v & =v_{F} \sqrt{1-\frac{3 a^{2} J_{z}}{v_{F}^{2} \pi^{2}}-\frac{2 a J_{z}}{\pi v_{F}}} \\
g & =\frac{J_{z}}{2 \pi^{2} a} .
\end{aligned}
$$

Al igual que en el caso del modelo de Hubbard, podemos calcular las dimensiones de escala del operador coseno

$$
\Delta=2\left(\frac{1-\frac{3 a J_{z}}{\pi v_{F}}}{1+\frac{a J_{z}}{\pi v_{F}}}\right)^{1 / 2}
$$

Más allá de que uno pueda observar la dependencia en $J_{z}$, tiene que recordar que el resultado es valido únicamente a bajas energías, y por lo tanto para valores pequeños de $J_{z}$. La dependencia exacta de $K$ en términos de $J_{z}$, se puede obtener comparando con el resultado del ansatz de Bethe en el rango $-J<J_{z} \leq J$ [45], dado por:

$$
K=\frac{\pi}{2(\pi-\theta)}, \quad v=\frac{\pi}{2} \frac{\sin \theta}{\theta}
$$

donde $\cos \theta=J_{z}$, y para $J_{z}$ pequeño, recuperamos la expresión (2.51).

En la figura (2.3) se compara el parámetro de Luttinger $K$ en función de $J_{z}$ obtenido mediante bosonización (ecuación (2.49)) y ansatz de Bethe. Como paso final mencionamos que 


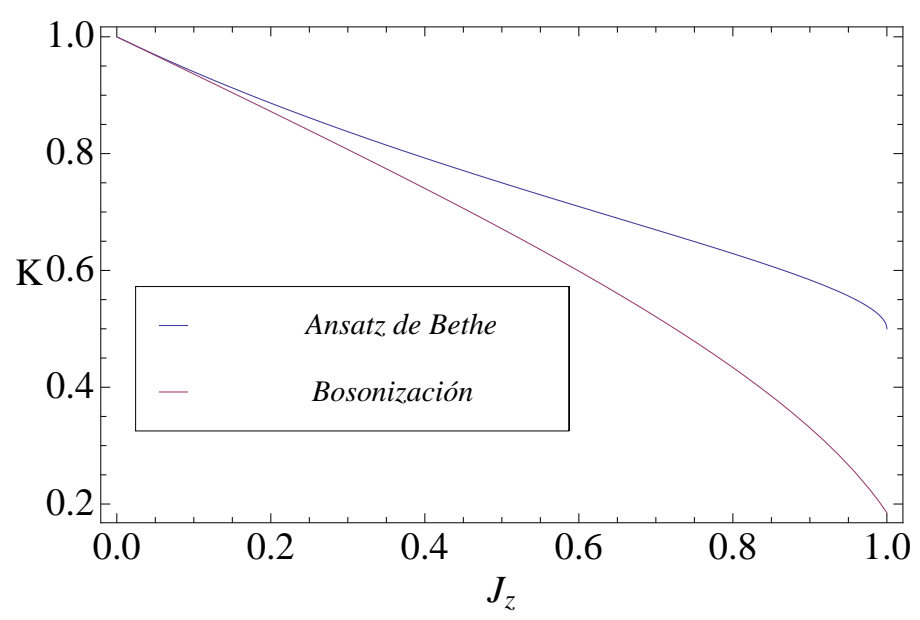

Figura 2.3: En esta figura se compara la dependencia del parámetro de Luttinger $K$ en función de $J_{z}(J=1)$, obtenido mediante bosonización con el resultados exacto del ansatz de Bethe.

utilizando la transformación de Jordan-Wigner (2.41) y las reglas de bosonización (2.10), (2.22) es fácil obtener el mapeo en el límite continuo entre los operadores de espín y los campos bosónicos [45],

$$
\begin{aligned}
S^{z}\left(x_{j}\right) & =\frac{1}{\sqrt{\pi}} \partial_{x_{j}} \varphi+a: \cos \left(2 k_{F} x_{j}+\sqrt{4 \pi} \varphi\right):+\frac{\langle M\rangle}{2} \\
S^{ \pm}\left(x_{j}\right) & =(-1)^{x}: e^{ \pm i \sqrt{4 \pi} \theta}\left(b \cos \left(2 k_{F} x_{j}+\sqrt{4 \pi} \varphi\right)+c\right):
\end{aligned}
$$

donde $k_{F}$ depende del llenado de los fermiones de Jordan-Wigner (ecuación (2.41)) y por lo tanto de la magnetización del sistema

$$
k_{F}=\frac{\pi}{2}(1-\langle M\rangle) .
$$

En la ecuación (2.55), : : indica orden normal con respecto al estado fundamental con magnetización $\langle M\rangle$, que deja el término constante en (2.55). El prefactor $\frac{1}{2}$ surge de la normalización de la magnetización con respecto a saturación $\langle M\rangle= \pm 1$. Utilizando las reglas de bosonización es posible obtener expresiones de las constantes $a, b$ y $c$ en términos de las constantes microscópicas del modelo, sin embargo las expresiones que se obtengan son solo aproximaciones de baja energía y la dependencia a toda escala de energía no puede ser obtenida por éste camino. Por esta razón, estas constantes se dice que son constantes no universales y pueden ser determinadas de manera indirecta y numéricamente a partir de varias funciones de correlación. Utilizando (2.55) es fácil mostrar que la densidad de energía en el límite continuo toma la forma

$$
\begin{aligned}
\lim _{\left|x_{j}-x_{i}\right| \rightarrow 0} \mathbf{S}\left(x_{i}\right) \mathbf{S}\left(x_{j}\right) \rightarrow \rho(x) \simeq & \alpha_{1} \partial_{x} \varphi+\beta_{1}: \cos \left(2 k_{F} x+\sqrt{4 \pi} \varphi\right): \\
& +\alpha_{2}\left(\partial_{x} \varphi\right)^{2}+\beta_{2}: \cos \left(4 k_{F} x+2 \sqrt{4 \pi} \varphi\right):+\ldots
\end{aligned}
$$


las constantes $\alpha^{\prime}$ s y $\beta^{\prime} s$ dependen del valor de la magnetización $\langle M\rangle$, mientras que los puntos ... indican armónicos superiores que pueden surgir como consecuencia de las interacciones del modelo [46], y en algunas situaciones deberán ser tenidos en cuenta.

\subsection{Bosonización de los operadores de espín, $d>1$.}

En esta sección describimos en detalle dos métodos analíticos que permiten estudiar sistemas de espines en dimensiones $d>1$. Introducimos las representaciones de bosones de Holstein-Primakoff y bosones de Schwinger para los operadores de espín. La primera representación, es de gran utilidad para describir fluctuaciones cuánticas de fases magnéticamente ordenadas, mientras que la segunda se utiliza para describir tanto fases ordenadas como desordenadas.

En el capítulo 5, estas técnicas nos permitirán estudiar el estado fundamental del modelo de Heisenberg antiferromagnético en redes frustradas.

\subsubsection{Bosones de Holstein-Primakoff y ondas de espín}

El operador de espín es un operador vectorial. En una fase con simetría SU(2) rota, al menos una de sus componentes tiene valor medio no nulo. Es natural describir la fase ordenada en términos de pequeñas fluctuaciones de los espines alrededor de sus valores de expectación. Lo que acabamos de comentar es la base de la teoría de ondas de espín. Para comenzar, consideremos el caso del hamiltoniano de Heisenberg ferromagnético,

$$
H=-J \sum_{\langle m, n\rangle} \mathbf{S}_{m} \cdot \mathbf{S}_{n}
$$

donde $J>0,\langle m, n\rangle$ denota que la sumatoria se realiza entre sitios vecinos de una red y $S_{j}^{a}$ representa la a-ésima componente del operador de espín en el sitio $j$, que satisfacen el álgebra

$$
\left[S_{j}^{a}, S_{k}^{b}\right]=i \in^{a b c} S_{j}^{c} \delta_{j k}
$$

con $a, b, c$ índices que toman los valores $x, y, z$ y $j, k$ índices de red.

Ahora, debido a la positividad de la constante de acoplamiento $J$, el hamiltoniano favorece configuraciones donde los espines en sitios vecinos están alineados en la misma dirección (ver figura 2.4a). Un posible estado fundamental del sistema está dado por

$$
|\Omega\rangle \equiv \otimes_{m}\left|\uparrow_{m}\right\rangle
$$

donde $\left|\uparrow_{m}\right\rangle$ representa un estado con máxima componente de $z$ del espín: $S_{m}^{z}\left|\uparrow_{m}\right\rangle=S\left|\uparrow_{m}\right\rangle$. Hemos escrito "un" estado fundamental en vez "del" estado fundamental, porque el sistema es altamente degenerado: un cambio simultáneo de la orientación de todos los espines no cambia la energía del estado fundamental, es decir el sistema posee una simetría global. Esto 
52 Bosonización de los operadores de espín, $d>1$.

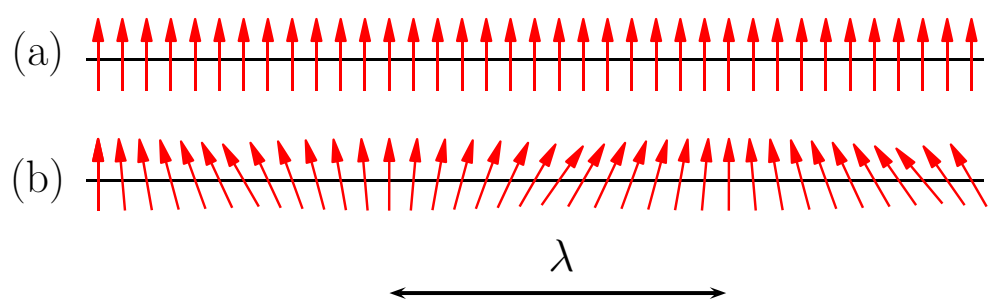

Figura 2.4: (a) estado fundamental ferromagnético "perfectamente alineado" . (b) Excitaciones de baja energía que surgen como fluctuaciones de larga longitud de onda.

se puede observar calculando el valor de expectación del hamiltoniano (2.58) en los estados $|\Omega\rangle$ y $|\Theta\rangle$ definidos como

$$
|\Theta\rangle=\exp (i \Theta . \mathbf{S})|\Omega\rangle
$$

donde $\mathbf{S}$ es el operador de espín total $\mathbf{S}=\sum_{m} \mathbf{S}_{m}$, y $\Theta$ es un vector de tres componentes que define los ángulos en las tres direcciones. Las excitaciones corresponden a fluctuaciones de longitud de onda $\lambda$ respecto de la configuración clásica (figura 2.4b). Para explorar cualitativamente la física de estas "ondas de espín", vamos a adoptar una imagen clásica, donde el espín $S \gg 1$. En este límite, la rotación de los espines alrededor de la configuración del estado fundamental es equivalente a una rotación "clásica" de momentos magnéticos.

En el límite de $S$ grande, y en el caso de excitaciones de baja energía, es natural describir la fase ordenada en términos de pequeñas fluctuaciones de espín alrededor de su valor de expectación. Para llevar a cabo esto, Holstein y Primakoff desarrollaron una representación de los operadores de espín en términos de operadores bosónicos de aniquilación y creación $b$ y $b^{\dagger}$ :

$$
\begin{aligned}
S_{j}^{+} & =\sqrt{2 S}\left(1-\frac{b_{j}^{\dagger} b_{j}}{2 S}\right)^{1 / 2} b_{j} \\
S_{j}^{-} & =\sqrt{2 S} b_{j}^{\dagger}\left(1-\frac{b_{j}^{\dagger} b_{j}}{2 S}\right)^{1 / 2} \\
S_{j}^{z} & =S-b_{j}^{\dagger} b_{j}
\end{aligned}
$$

Usando la relación de conmutación de los operadores bosónicos, $\left[b_{i}, b_{j}^{\dagger}\right]=\delta_{i, j}$, es fácil mostrar que los operadores de espín (2.62) obedecen las relaciones de conmutación de SU(2) (ecuación (2.59)).

El espacio físico de los operadores, es solo un subespacio del espacio de Fock de los operadores $b$ y está generado por los estados

$$
\left\{\left|n_{b}\right\rangle\right\}=\{|0\rangle,|1\rangle \ldots|2 S\rangle\}
$$

donde $n_{b}$ es el número de bosones y satisface $b^{\dagger} b\left|n_{b}\right\rangle=n_{b}\left|n_{b}\right\rangle$. Es necesario introducir la restricción no-holonómica $0 \leq n_{b} \leq 2 S$ para poder mapear correctamente los estados físicos 
de espín en los bosónicos. La utilidad de esta representación es clara cuando el valor del espín es grande. El procedimiento usual consiste en expandir las raíces en potencias de $1 / S$ $(2.62)$,

$$
\sqrt{2 S}\left(1-\frac{b_{j}^{\dagger} b_{j}}{2 S}\right)^{1 / 2}=\sqrt{2 S}\left(1-\frac{b_{j}^{\dagger} b_{j}}{4 S}-\frac{\left(b_{j}^{\dagger} b_{j}\right)^{2}}{32 S^{2}} \ldots\right)
$$

Insertando (2.64) en el hamiltoniano de Heisenberg ferromagnético, y ordenando en potencias de $1 / S$ se obtiene

$$
\begin{aligned}
\mathcal{H} & =-J S^{2} \sum_{\langle m, n\rangle}\left\{S_{m}^{z} S_{n}^{z}+\frac{1}{2}\left(S_{m}^{+} S_{n}^{-}+S_{m}^{-} S_{n}^{+}\right)\right\} \\
\mathcal{H} & =-J S^{2} \sum_{m n}\left\{1+\frac{1}{S} \hat{h}_{1}\left[b^{\dagger}, b\right]+\frac{1}{S^{2}} \hat{h}_{2}\left[b^{\dagger}, b, b^{\dagger}, b\right]+O\left(\frac{1}{S^{3}}\right)\right\}
\end{aligned}
$$

donde $\hat{h}_{1}$ y $\hat{h}_{2}$ son términos cuadráticos y cuárticos en los operadores bosónicos. Así, al orden más bajo $(1 / S)$ se obtiene un hamiltoniano cuadrático de "ondas de espín" no interactuantes que se puede diagonalizar mediante una transformación para-unitaria (ver detalles en el apéndice B). En el caso de una cadena periódica de $N$ sitios, con interacciones a primeros vecinos, es fácil demostrar que el hamiltoniano diagonal queda

$$
\mathcal{H}=-J N S^{2}+S^{2}\left(\sum_{k \in B Z} w_{k} b_{k}^{\dagger} b_{k}+O\left(\frac{1}{S^{2}}\right) \ldots\right)
$$

donde $S^{2} w_{k}=4 J S \sin ^{2}(k / 2)$. En particular, en el límite $k \rightarrow 0$, la energía de las excitaciones elementales se anulan. Estas excitaciones de baja energía, se conocen como magnones y describen las excitaciones de espín de un ferromagneto. Los términos de orden superior introducen interacciones entre los magnones. El truncamiento del desarrollo trae como consecuencia acoplar subespacios físicos con no físicos. Sin embargo, la aproximación lineal de ondas de espín ha demostrado tener un notable éxito para estudiar fases ordenadas tanto del hamiltoniano de Heisenberg ferro- como antiferromagnético.

Ahora veamos lo que ocurre en el caso del hamiltoniano de Heisenberg Antiferromagnético $(\mathrm{AF})$ :

$$
H=J \sum_{\langle m, n\rangle} \mathbf{S}_{m} \cdot \mathbf{S}_{n}
$$

donde $J>0$.

Más allá de que el hamiltoniano AF "solo" difiera del ferromagnético en el signo de la constante de acoplamiento, la diferencia en la física del problema es drástica. Primero, la fenomenología desarrollada por el AF depende sensiblemente de la gometría de la red en la cual se encuentra: por ejemplo en el caso del modelo clásico sobre una red bipartita como la red cuadrada (figura 2.5a), el estado fundamental del modelo de Heisenberg AF, adopta una configuración alternada, conocida como "estado de Néel", donde todos los espines vecinos están "orientados" de manera antiparalela. En contraste, en redes no bipartitas tales como 


\section{Bosonización de los operadores de espín, $d>1$.}

la triangular mostrada en la figura 2.5b , no es posible encontrar un arreglo de espines en donde cada uno de los bonds recupere la energía total $J$. Los modelos de espines de esta clase se denominan Frustrados (ver sección 1.3.4).

Volviendo al caso de la cadena unidimensional, podemos notar que la cadena es trivialmente

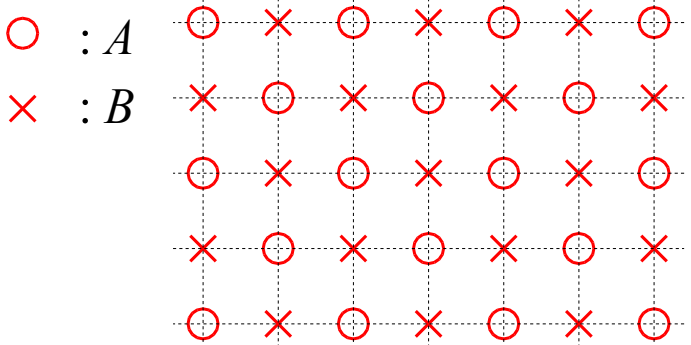

a)

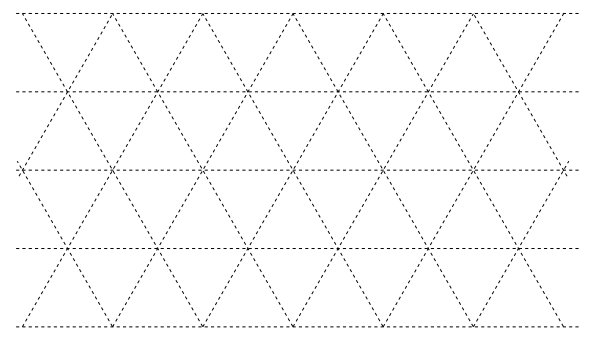

b)

Figura 2.5: (a) Ejemplo de una red bidimensional bipartita. (b) red no bipartita.

bipartita y por lo tanto el estado fundamental clásico corresponde al de Néel (figura 2.6a). Más allá de que este no es un autoestado del hamiltoniano cuántico, cuando $S \gg 1$ el estado fundamental clásico sirve como un estado de referencia de manera tal que las fluctuaciones cuánticas pueden ser examinadas con la representación de Holstein-Primakoff. Antes de

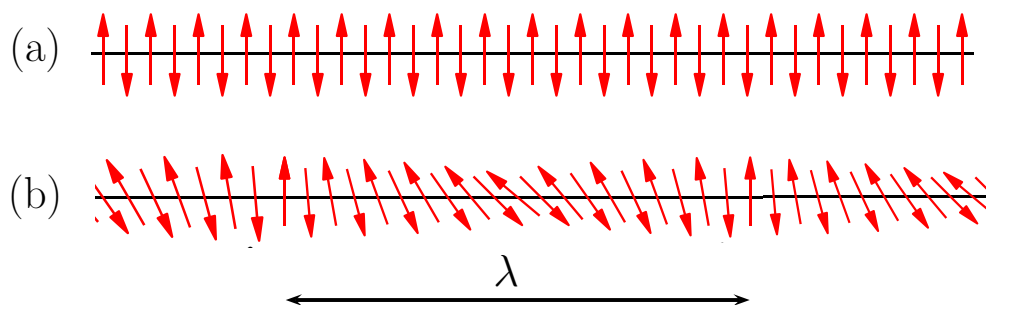

Figura 2.6: (a) Configuración tipo Néel de una cadena de espines. (b) Imagen ilustrativa de una onda de espín antiferromagnética.

expandir el hamiltoniano en términos de los operadores bosónicos, es conveniente aplicar una transformación canónica al hamiltoniano en que los espines en una subred, digamos $B$, son rotados $180^{\circ}$ alrededor de un eje, por ejemplo $S_{B}^{x} \rightarrow \tilde{S}_{B}^{x}=S_{B}^{x}, S_{B}^{y} \rightarrow \tilde{S}_{B}^{y}=-S_{B}^{y}$ y $S_{B}^{z} \rightarrow \tilde{S}_{B}^{z}=-S_{B}^{z}$. De esta manera, cuando representamos en término de los operadores bosónicos, el estado de Néel equivale a un estado ferromagnético, con todos los espines alineados. Es de esperar que una distorsión gradual de éste estado producirá el análogo antiferromagnético de las ondas de espín como en el caso ferromagnético.

Representado en términos de los operadores transformados, el hamiltoniano queda

$$
\mathcal{H}=-J S^{2} \sum_{\langle m, n\rangle}\left\{S_{m}^{z} \tilde{S}_{n}^{z}-\frac{1}{2}\left(S_{m}^{+} \tilde{S}_{n}^{+}+S_{m}^{-} \tilde{S}_{n}^{-}\right)\right\}
$$


Así, aplicando la transformación de Holstein-Primakoff en la aproximación cuadrática obtenemos el hamiltoniano:

$$
\mathcal{H}=-J N S^{2}+J S^{2}\left(\sum_{\langle m, n\rangle} \frac{1}{S}\left(b_{m}^{\dagger} b_{m}+b_{n}^{\dagger} b_{n}+b_{m} b_{m}+b_{m}^{\dagger} b_{m}^{\dagger}\right)+O\left(\frac{1}{S^{2}}\right)\right)
$$

después de una transformación de Fourier, $b_{m}=N^{-1 / 2} \sum_{k} e^{i k x_{m}} b_{k}$, éste toma la forma

$$
\mathcal{H}=-J N S(S+1)+J S^{2}\left(\sum_{\langle m, n\rangle} \frac{1}{S}\left(b_{k}^{\dagger}, b_{-k}\right)\left[\begin{array}{cc}
1 & \gamma_{k} \\
\gamma_{k} & 1
\end{array}\right]\left(\begin{array}{c}
b_{k} \\
b_{-k}^{\dagger}
\end{array}\right)+O\left(\frac{1}{S^{2}}\right)\right)
$$

donde $\gamma_{k}=\cos (k)$. Para remover el término $b^{\dagger} b^{\dagger}$, realizamos una transformación de Bogoliubov $^{4}[4]$

$$
\tilde{b}_{k}=\cosh \left(\theta_{k}\right) b_{k}-\sinh \left(\theta_{k}\right) b_{-k}^{\dagger}
$$

De esta manera, el hamiltoniano (a primer orden en $1 / S$ ) queda

$$
\mathcal{H}=-N J S^{2}+2 J S^{2}\left(\sum_{k} \frac{1}{S}|\sin (k)|\left[\tilde{b}_{k}^{\dagger} \tilde{b}_{k}+\frac{1}{2}\right]\right)
$$

Cabe notar, en contraste con el caso ferromagnético, que las excitaciones de de ondas de espín en el modelo antiferromagnético revelan un comportamiento lineal en el límite $k \rightarrow 0$.

\subsubsection{Método de Bosones de Schwinger}

En la representación de Schwinger [47] cada variable de espín es reemplazada por dos bosones $b_{i, \sigma}(\sigma=\uparrow, \downarrow)$ de acuerdo a:

$$
\mathbf{S}_{i}=\frac{1}{2} \mathbf{b}_{i}^{\dagger} \cdot \sigma \cdot \mathbf{b}_{i}
$$

donde $\sigma=\left(\sigma^{x}, \sigma^{y}, \sigma^{z}\right)$ son las matrices de Pauli y $\mathbf{b}_{i}=\left(\begin{array}{c}b_{i, \uparrow} \\ b_{i, \downarrow}\end{array}\right)$.

Las componentes del espín se escriben entonces

$$
\begin{aligned}
S_{i}^{z} & =\frac{1}{2}\left(b_{i, \uparrow}^{\dagger} b_{i, \uparrow}-b_{i, \downarrow}^{\dagger} b_{i, \downarrow}\right), \\
S_{i}^{+} & =b_{i, \uparrow}^{\dagger} b_{i, \downarrow}, \\
S_{i}^{-} & =b_{i, \downarrow}^{\dagger} b_{i, \uparrow} .
\end{aligned}
$$

\footnotetext{
${ }^{4}$ Este tipo de transformaciones es frecuentemente aplicada en magnetismo cuántico, superconductividad, y en todos los problemas donde el número de partículas no es una cantidad conservada.
} 
56 Bosonización de los operadores de espín, $d>1$.

Es fácil verificar que tal definición, satisface el álgebra de $S U(2)$ (ecuación (2.59)). La magnitud del espín $S$ define el subespacio físico

$$
\left\{\left|n_{\uparrow}, n_{\downarrow}\right\rangle: n_{\uparrow}+n_{\downarrow}=2 S\right\} .
$$

El subespacio está dado por la restricción holonómica

$$
\left(b_{i, \uparrow}^{\dagger} b_{i, \uparrow}+b_{i, \downarrow}^{\dagger} b_{i, \downarrow}-2 S\right)=0
$$

En el espacio proyectado, la magnitud del espín está bien definida,

$$
\mathbf{S}^{2}=S(S+1)
$$

mientras que los estados de espín (espín $S$ y proyección $m_{s}$ ) se obtienen de los estados bosónicos según [4]

$$
\left|S, m_{s}\right\rangle_{i}=\frac{\left(b_{i, \uparrow}^{\dagger}\right)^{S+m_{s}}\left(b_{i, \downarrow}^{\dagger}\right)^{S-m_{s}}}{\sqrt{\left(S+m_{s}\right) !\left(S-m_{s}\right) !}}|0\rangle .
$$

Los bosones de Schwinger tienen, por construcción, asociada una invariancia de gauge $b_{i, \sigma} \rightarrow$ $b_{i, \sigma} e^{i \theta_{i}}$, que no modifica el valor de los observables.

Al utilizar el mapeo (2.73) y la restricción (2.75), el hamiltoniano bosónico que se obtiene a partir del hamiltoniano de Heisenberg, presenta términos cuárticos en operadores bosónicos siendo necesario realizar alguna aproximación para poder tratar este problema.

\section{Aproximación de campo medio}

Hay varias maneras de formular una teoría de campo medio a partir de un hamiltoniano bosónico cuártico: bien puede ser en el contexto de las teorías llamadas large- $\mathcal{N}$, o como un enfoque variacional. Nosotros vamos a utilizar el enfoque variacional en el capítulo 5; sin embargo antes de desarrollar el método, vamos a comentar las ideas del primer enfoque, las cuales, de alguna manera, justifican el segundo.

Las teorías llamadas large- $\mathcal{N}$, generalizan el modelo de interés a una familia de modelos donde el número de grados de libertad internos se caracteriza por un número entero $\mathcal{N}$. El límite $\mathcal{N} \rightarrow \infty$, conduce a un modelo que puede ser resuelto más fácilmente, y donde las propiedades de $\mathcal{N}$ finito, pueden ser obtenidas a partir de una expansión en serie de potencias de $1 / \mathcal{N}$.

Estas teorías son bien conocidas en la física de partículas, y en teorías de fermiones pesados $[48,49,50]$. Estas fueron introducidas en magnetismo cuántico por Affleck y Marston [51, 52], que usaron una representación para los operadores de espín en términos de fermiones para tratar los modelos de Hubbard y Heisenberg (de espín $S=1 / 2$ ) al extender la simetría $\mathrm{SU}(2)$ a $\mathrm{SU}(\mathcal{N})$, preservando la invariancia rotacional del hamiltoniano bajo $\mathrm{SU}(\mathcal{N})$. Desde entonces, otras extensiones de $\mathrm{SU}(2)$ han sido usadas, incluyendo $\operatorname{Sp}(\mathcal{N})$ [53]. Las teorías large- $\mathcal{N}$ fermiónicas capturan el límite cuántico extremo, $S / \mathcal{N} \ll 1$, donde el estado fundamental es siempre desordenado. Estas son utilizadas para estudiar las posibles fases desordenadas del estado fundamental, pero no para determinar si un modelo particular presenta una fase 
desordenada. Para ello, uno necesita una representación bosónica de los operadores de espín, donde el órden de largo alcance corresponde a la condensación de los bosones en un vector de onda particular k. Arovas y Auerbach introdujeron la teoría $\mathrm{SU}(\mathcal{N})$ bosónica [54], que puede tratar valores arbitrarios de $S / \mathcal{N}$, y tanto estados magnéticos ordenados como desordenados. Esta teoría fue un gran éxito para describir ferromagnetos y antiferromagnetos bipartitos, pero incapaz para tratar magnetos frustrados. Para resolver esto, Sachdev y Read extendieron la teoría para acoplamientos arbitrarios antiferromagnéticos por limitar la invariancia rotacional al grupo $\operatorname{Sp}(\mathcal{N})$ [55]. Sin embargo, ninguna de estas teorías son invariantes frente a inversión temporal. Recientemente R. Flint y P. Coleman [56] desarrollaron una nueva generalización del modelo de Heisenberg al que llamaron symplectic- $\mathcal{N}$.

En todas estas teorías el límite de large- $\mathcal{N}$ constituye una manera de obtener una teoría de campo medio que es controlada por el parámetro $1 / \mathcal{N}$.

Como mencionamos antes, en esta tesis vamos a usar el enfoque variacional guiados por [56] que nos va a proporcionar una manera unificada para estudiar el efecto de diferentes interacciones.

Consideremos el hamiltoniano de Heisenberg

$$
\mathcal{H}=\sum_{\langle i j\rangle} J_{i, j} \mathbf{S}_{\mathbf{r}_{i}} \cdot \mathbf{S}_{\mathbf{r}_{j}}
$$

En términos de los bosones de Schwinger, la interacción espín-espín queda

$$
\mathbf{S}_{\mathbf{r}_{i}} \cdot \mathbf{S}_{\mathbf{r}_{j}}=\frac{1}{4} b_{\mathbf{r}_{i}, \sigma}^{\dagger} \sigma_{\sigma, \sigma^{\prime}} b_{\mathbf{r}_{i}, \sigma^{\prime}} b_{\mathbf{r}_{j}, \tau}^{\dagger} \sigma_{\tau, \tau^{\prime}} b_{\mathbf{r}_{j}, \tau^{\prime}}
$$

con la restricción del número de bosones en cada sitio

$$
\sum_{\sigma} b_{\mathbf{r}_{i}, \sigma}^{\dagger} b_{\mathbf{r}_{i}, \sigma}=2 S
$$

Dado que el hamiltoniano de Heisenberg tiene simetría $S U(2)$, necesitamos obtener una descomposición de campo medio que conserve explícitamente el carácter invariante rotacional del modelo. Para ello, definimos la siguiente matriz

$$
\Xi_{\mathbf{r}_{i}}=\left(\begin{array}{cc}
b_{\mathbf{r}_{i}, \uparrow} & -b_{\mathbf{r}_{i, \downarrow}}^{\dagger} \\
b_{\mathbf{r}_{i}, \downarrow} & b_{\mathbf{r}_{i}, \downarrow}^{\dagger}
\end{array}\right)
$$

Así definida, $\Xi_{\mathbf{r}_{i}}$ transforma ante rotaciones como $\Xi_{\mathbf{r}_{i}}^{\prime}=\mathbf{R} . \Xi_{\mathbf{r}_{i}}$, donde $\mathbf{R} \in S U(2)$. Utilizando las reglas de conmutación bosónicas y las propiedades de las matrices de Pauli se puede mostrar que

$$
\mathbf{S}_{\mathbf{r}_{i}} \cdot \mathbf{S}_{\mathbf{r}_{j}}=\frac{1}{8}: \operatorname{Tr}\left(\sigma_{z} \Xi_{\mathbf{r}_{i}}^{\dagger} \Xi_{\mathbf{r}_{j}} \sigma_{z} \Xi_{\mathbf{r}_{j}}^{\dagger} \Xi_{\mathbf{r}_{i}}\right):
$$

donde : : indica orden normal de operadores y $\sigma_{z}=\left(\begin{array}{cc}1 & 0 \\ 0 & -1\end{array}\right)$. Con esto, el acoplamiento espín-espín puede escribirse como

$$
\mathbf{S}_{\mathbf{r}_{i}} \cdot \mathbf{S}_{\mathbf{r}_{j}}=: \mathbf{B}_{\mathbf{r}_{i}, \mathbf{r}_{j}}^{\dagger} \mathbf{B}_{\mathbf{r}_{i}, \mathbf{r}_{j}}:-\mathbf{A}_{\mathbf{r}_{i}, \mathbf{r}_{j}}^{\dagger} \mathbf{A}_{\mathbf{r}_{i}, \mathbf{r}_{j}},
$$


donde ${ }^{5}$

$$
\mathbf{B}_{\mathbf{r}_{i}, \mathbf{r}_{j}}^{\dagger}=\frac{1}{2} \sum_{\sigma} b_{\mathbf{r}_{i}, \sigma}^{\dagger} b_{\mathbf{r}_{j}, \sigma}, \quad \mathbf{A}_{\mathbf{r}_{i}, \mathbf{r}_{j}}^{\dagger}=\frac{1}{2} \sum_{\sigma} \tilde{\sigma} b_{\mathbf{r}_{i}, \sigma}^{\dagger} b_{\mathbf{r}_{j},-\sigma}^{\dagger} .
$$

con $\sigma= \pm \mathrm{y}: \mathbf{O}$ : indicando orden normal de los operadores. El operador $\mathbf{A}_{\mathbf{r}_{i}, \mathbf{r}_{j}}^{\dagger}$ actuando sobre el vacío de Fock, crea un singlete de espín $1 / 2$ entre los sitios $\mathbf{r}_{i}$ y $\mathbf{r}_{j}$, es decir: $\mathbf{A}_{\mathbf{r}_{i}, \mathbf{r}_{j}}^{\dagger}|0\rangle=$ $\frac{1}{2}\left(\left|\uparrow_{i} \downarrow_{j}\right\rangle-\left|\downarrow_{i} \uparrow_{j}\right\rangle\right)$; mientras que $\mathbf{B}_{\mathbf{r}_{i}, \mathbf{r}_{j}}^{\dagger}$ crea un "enlace ferromagnético" $\mathbf{B}_{\mathbf{r}_{i}, \mathbf{r}_{j}}^{\dagger}|0\rangle=\frac{1}{2}\left(\mid \uparrow_{i}\right.$ $\left.\left.\uparrow_{j}\right\rangle+\left|\downarrow_{i} \downarrow_{j}\right\rangle\right)$. La interpretación física de los operadores de $\mathbf{A}^{\prime} s$ y $\mathbf{B}^{\prime} s$ es más evidente si escribimos estos en términos de los operadores de espín:

$$
\begin{aligned}
\mathbf{A}_{\mathbf{r}_{i}, \mathbf{r}_{j}}^{\dagger} \cdot \mathbf{A}_{\mathbf{r}_{i}, \mathbf{r}_{j}} & =\frac{1}{4}\left(\mathbf{S}_{\mathbf{r}_{i}}-\mathbf{S}_{\mathbf{r}_{j}}\right)^{2} \\
\mathbf{B}_{\mathbf{r}_{i}, \mathbf{r}_{j}}^{\dagger} \cdot \mathbf{B}_{\mathbf{r}_{i}, \mathbf{r}_{j}} & =\frac{1}{4}\left(\mathbf{S}_{\mathbf{r}_{i}}+\mathbf{S}_{\mathbf{r}_{j}}\right)^{2}
\end{aligned}
$$

así, el valor medio de $\mathbf{A}_{\mathbf{r}_{i}, \mathbf{r}_{j}}^{\dagger} \mathbf{A}_{\mathbf{r}_{i}, \mathbf{r}_{j}}$ mide el orden antiferromagnético mientras que el valor medio de $\mathbf{B}_{\mathbf{r}_{i}, \mathbf{r}_{j}}^{\dagger} \mathbf{B}_{\mathbf{r}_{i}, \mathbf{r}_{j}}$ mide el ferromagnético.

Para desacoplar la parte cuártica, efectuamos la siguiente descomposición de campo medio (MF) invariante rotacional:

$$
\left(\mathbf{S}_{\mathbf{r}_{i}} \cdot \mathbf{S}_{\mathbf{r}_{j}}\right)_{M F}=\frac{1}{4} \operatorname{Tr}\left(\Omega_{\mathbf{r}_{i}, \mathbf{r}_{j}} \Xi_{\mathbf{r}_{j}}^{\dagger} \Xi_{\mathbf{r}_{i}}\right)+\frac{1}{4} \operatorname{Tr}\left(\Xi_{\mathbf{r}_{i}}^{\dagger} \Xi_{\mathbf{r}_{j}} \Omega_{\mathbf{r}_{j}, \mathbf{r}_{i}}\right)-\frac{1}{2} \operatorname{Tr}\left(\Omega_{\mathbf{r}_{i}, \mathbf{r}_{j}} \sigma_{z} \Omega_{\mathbf{r}_{j}, \mathbf{r}_{i}} \sigma_{z}\right) .
$$

La matriz de parámetros de orden está dada por

$$
\Omega_{\mathbf{r}_{i}, \mathbf{r}_{j}}=\frac{1}{2} \sigma_{z}\left\langle\Xi_{\mathbf{r}_{i}}^{\dagger} \Xi_{\mathbf{r}_{j}}\right\rangle \sigma_{z}=\left(\begin{array}{cc}
B_{\mathbf{r}_{i}, \mathbf{r}_{j}} & A_{\mathbf{r}_{i}, \mathbf{r}_{j}} \\
-A_{\mathbf{r}_{i}, \mathbf{r}_{j}} & B_{\mathbf{r}_{i}, \mathbf{r}_{j}}
\end{array}\right),
$$

donde $A_{\mathbf{r}_{i}, \mathbf{r}_{j}}$ y $B_{\mathbf{r}_{i}, \mathbf{r}_{j}}$ son los valores medios de los operadores (2.83) tomados con la función de onda del estado fundamental. Finalmente, el hamiltoniano de Heisenberg se puede expresar de la forma

$$
\begin{aligned}
\mathcal{H}_{M F}= & \sum_{i j} J_{i j}\left(\mathbf{S}_{\mathbf{r}_{i}} \cdot \mathbf{S}_{\mathbf{r}_{j}}\right)_{M F} \\
= & \sum_{i j} J_{i j}\left(B_{\mathbf{r}_{i}, \mathbf{r}_{j}} \mathbf{B}_{\mathbf{r}_{i}, \mathbf{r}_{j}}^{\dagger}-A_{\mathbf{r}_{i}, \mathbf{r}_{j}} \mathbf{A}_{\mathbf{r}_{i}, \mathbf{r}_{j}}^{\dagger}+\text { h.c. }\right)-J_{i j}\left(\mathbf{S}_{\mathbf{r}_{i}} \cdot \mathbf{S}_{\mathbf{r}_{j}}\right)_{M F} \\
& +\sum_{i} \lambda_{i} \sum_{\sigma}\left(b_{\mathbf{r}_{i}, \sigma}^{\dagger} b_{\mathbf{r}_{i}, \sigma}-S\right)
\end{aligned}
$$

donde $\left(\mathbf{S}_{\mathbf{r}_{i}} \cdot \mathbf{S}_{\mathbf{r}_{j}}\right)_{M F}=\left|B_{\mathbf{r}_{i}, \mathbf{r}_{j}}\right|^{2}-\left|A_{\mathbf{r}_{i}, \mathbf{r}_{j}}\right|^{2}$.

En el último término, $\lambda_{i}$ es un multiplicador de Lagrange que impone, en valor medio, la restricción del número de bosones (2.75) en cada sitio de la red. En general, este término dificulta los cálculos tanto numéricos como analíticos y comúnmente se implementa una aproximación que permite simplificar el problema. Esta consiste en relajar la restricción de

\footnotetext{
${ }^{5} \mathrm{El}$ tilde en una letra griega como $\tilde{\alpha}$ indica que un término como $\sum_{\alpha} \tilde{\alpha} \mathbf{O}_{\alpha} \mathbf{O}_{-\alpha}=\sum_{\alpha}\left(\mathbf{O}_{\alpha} \mathbf{O}_{-\alpha}-\mathbf{O}_{-\alpha} \mathbf{O}_{\alpha}\right)$
} 
local a global, $\lambda_{i} \equiv \lambda$. En el caso de que la red esté formada por varias subredes (esto equivale a que hay varios sitios por celda unidad), la restricción se hace global a cada subred $\lambda_{i} \equiv \lambda_{a}$, $\lambda_{j} \equiv \lambda_{b}, \lambda_{k} \equiv \lambda_{c} \ldots$ con $i \in \operatorname{subred} A, j \in \operatorname{subred} B, k \in$ subred $C$...etc.

El problema que nos queda es el de un hamiltoniano cuadrático en bosones con parámetros que deben calcularse de manera autoconsistente

$$
\begin{gathered}
A_{\mathbf{r}_{i}, \mathbf{r}_{j}}=\left\langle\mathbf{A}_{\mathbf{r}_{i}, \mathbf{r}_{j}}\right\rangle \\
B_{\mathbf{r}_{i}, \mathbf{r}_{j}}=\left\langle\mathbf{B}_{\mathbf{r}_{i}, \mathbf{r}_{j}}\right\rangle .
\end{gathered}
$$

sujetos a la condición

$$
\left\langle b_{\mathbf{r}_{i}, \uparrow}^{\dagger} b_{\mathbf{r}_{i}, \uparrow}+b_{\mathbf{r}_{i}, \downarrow}^{\dagger} b_{\mathbf{r}_{i}, \downarrow}\right\rangle=2 S
$$

Debido a la presencia de términos que no conservan el número de bosones el hamiltoniano debe diagonalizarse mediante una transformación de Bogoliubov (ver apéndice B).

En el capítulo 5 utilizaremos tanto el mapeo a bosones Holstein-Primakoff como el de bosones de Schwinger para estudiar las fases del estado fundamental en dos modelos de sistemas bidimensionales: el hamiltoniano de Heisenberg sobre la red de kagomé y la red hexagonal.

\subsection{Métodos Numéricos}

En esta sección abordaremos dos técnicas numéricas que en los últimos años han mostrado ser de gran utilidad en una amplia gama de problemas de materia condensada; en particular en sistemas fuertemente correlacionados. Estas técnicas son: Lanczos [6] y DMRG (Density Matrix Renormalization Group) [57]. Estas son imparciales en el sentido que no asumen ningún tipo de naturaleza para el estado fundamental del sistema permitiendo estudiar modelos como el de Heisenberg, el $t-J$ y el de Hubbard, entre otros. Sin embargo, cada uno de estas técnicas tienen sus limitaciones: la más importante de todas es que con estas se pueden estudiar sistemas con un número finito de sitios.

En ambas técnicas, la idea central consiste en construir una representación matricial del hamiltoniano para un dado número de sitios en una dada base apropiada y posteriormente diagonalizar la matriz mediante algún algoritmo apropiado. Una vez diagonalizado el hamiltoniano se pueden calcular distintas cantidades como correlaciones espín-espín de carga, ...etc. Para entender por qué estas técnicas son necesarias para estudiar los modelos mencionados, supongamos el modelo de Heisenberg en una red de $L$ sitios. Cada sitio tiene dos posibles estados: espín $\uparrow \mathrm{y} \downarrow$. Una red con $L$ sitios tiene $2^{L}$ estados y esta es la dimensión de la matriz del hamiltoniano. Similarmente, para el modelo $t-J$ y de Hubbard tenemos $3^{L}$ y $4^{L}$ estados, respectivamente. Debido al comportamiento exponencial con $L$, incluso en redes pequeñas, el hamiltoniano es demasiado grande para poder ser manejado en las computadoras de hoy en día.

Una manera de reducir las dimensiones de trabajo es a través de las simetrías del modelo. Muchos modelos exhiben la conservación del espín total, componente $z$ del espín total, carga total, número de partículas, simetrías de traslación y rotación, etc. Es decir,

$$
\left[\mathcal{H}, \mathbf{S}_{\text {total }}^{2}\right]=\left[\mathcal{H}, S_{\text {total }}^{z}\right]=[H, \mathbf{N}]=0
$$


donde $\mathcal{H}$ es el hamiltoniano y

$$
\mathbf{S}_{\text {total }}=\sum_{i} \mathbf{S}_{i}, \quad \mathbf{N}=\sum_{i} n_{i}
$$

Por otra parte, estos operadores también pueden conmutar entre si,

$$
\left[\mathbf{S}_{\text {total }}^{2}, S_{\text {total }}^{z}\right]=\left[S_{\text {total }}^{z}, \mathbf{N}\right]=\left[\mathbf{S}_{\text {total }}^{2}, \mathbf{N}\right]=0 .
$$

En ese caso, los autovalores de $\mathcal{H}, \mathbf{S}_{\text {total }}, S_{\text {total }}^{z}$ y $\mathbf{N}$ son simultáneamente buenos números cuánticos (denotados por $E, S(S+1), S^{z}$ y $N$ respectivamente). En un dado tratamiento numérico de un dado modelo es posible considerar autoestados que simultáneamente diagonalizen todos los operadores asociados con sus simetrías. La manera más eficiente de aprovechar esto, es trabajar en una base en que los operadores asociados a las simetrías son siempre diagonales, seleccionando un subespacio o sector del espacio de Hilbert con un determinado conjunto de autovalores de esos operadores, y diagonalizar $\mathcal{H}$ en ese sector particular. La diagonalización se realiza luego dentro de cada uno de estos bloques lo cual implica lógicamente un menor esfuerzo.

Finalmente si uno realiza el estudio con distintos tamaños, lo cual va a depender de las características del sistema, puede extrapolar los resultados al límite termodinámico. Este procedimiento llamado escaleo de tamaño finito [58] es especialmente utilizado regímenes cercanos a transiciones de fase puesto que en ese caso, las cantidades físicas poseen una fuerte dependencia en el tamaño del sistema [59]. Sin embargo, mediante este procedimiento es posible extraer una gran información del límite termodinámico examinando unos pocos tamaños.

\subsubsection{Algoritmo de Lanczos}

La idea básica del algoritmo de Lanczos $[60,61]$ es que se puede construir una base especial donde la matriz $H$ del hamiltoniano tiene una representación tridiagonal. Así, el estado fundamental puede ser calculado mediante técnicas eficientes para este tipo de matrices. La matriz tridiagonal es construida iterativamente. Primero se construye un vector normalizado arbitrario $|\phi\rangle$ del espacio de Hilbert de dimensión $N_{h}$, luego bajo la aplicación sucesiva del hamiltoniano, se construye el conjunto de vectores

$$
\left\{|\phi\rangle, H|\phi\rangle, H^{2}|\phi\rangle, \ldots, H^{N_{k}}|\phi\rangle\right\}
$$

Este conjunto de vectores pertenece a un subespacio, denominado subespacio de Krylov de orden $N_{k}$, del espacio de Hilbert. Una base posible de este subespacio se obtiene ortonormalizando los vectores del conjunto generado mediante la siguiente relación de recurrencia

$$
\left|q_{i+1}\right\rangle=H\left|q_{i}\right\rangle-\alpha_{i}\left|q_{i}\right\rangle-\beta_{i}\left|q_{i-1}\right\rangle
$$




$$
\begin{gathered}
\alpha_{i}=\left\langle q_{i}|H| q_{i}\right\rangle \\
\beta=\left\|q_{i}\right\|
\end{gathered}
$$

Los elementos de la base del subespacio de Krylov se pueden expresar en forma matricial como

$$
Q^{N_{h} \times N_{k}}=\left[\left|q_{1}\right\rangle, \ldots,\left|q_{N_{k}}\right\rangle\right]
$$

en donde hemos simplificado la notación sobrentendiendo que cada columna está compuesta por las componentes de $\left|q_{i}\right\rangle$ en la base en la que esta expresado el hamiltoniano. En esta base, la representación matricial $H$ del hamiltoniano resulta tridiagonal. Esta transformación se obtiene realizando la siguiente operación unitaria de cambio de base

$$
H_{T D}^{\left(N_{k} \times N_{k}\right)}=Q^{T\left(N_{k} \times N_{h}\right)} H^{\left(N_{h} \times N_{h}\right)} Q^{\left(N_{h} \times N_{k}\right)}
$$

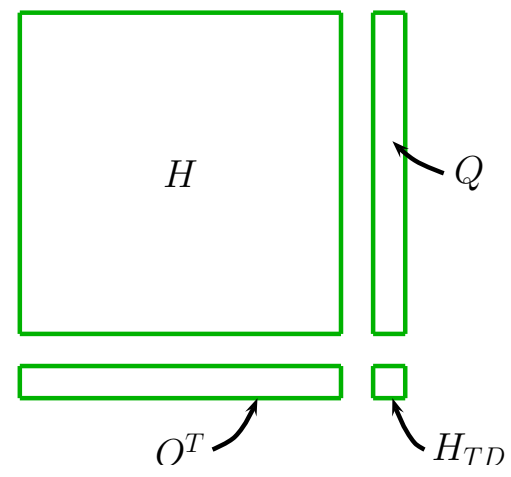

Figura 2.7: Reducción de la matriz del hamiltoniano.

Esta transformación está esquematizada en figura 2.7. La matriz tridiagonal $H_{T D}^{\left(N_{k} \times N_{k}\right)}$, que puede ser diagonalizada fácilmente para obtener el autovalor, y autovector correspondiente, del estado fundamental, posee la forma

$$
H_{T D}=\left(\begin{array}{cccccc}
\alpha_{1} & \beta_{1} & & & & \\
\beta_{1} & \alpha_{2} & \beta_{2} & & & \\
& \beta_{2} & \alpha_{3} & \beta_{3} & & \\
& & \beta_{3} & . & . & \\
& & & . & . & .
\end{array}\right)
$$

En cada iteración de método de Lanczos el subespacio de Krylov es ampliado hasta que la diferencia en la energía fundamental entre dos iteraciones sucesivas sea menor que una tolerancia impuesta, lo que significa que se ha convergido al estado fundamental. Si bien 
depende de cada problema en particular, el número de iteraciones requeridos para lograr convergencia al autovalor más bajo con una precisión de $10^{12}$ es típicamente del orden de una centena.

\subsubsection{Método de Renormalización de la Matriz Densidad: DMRG}

En la sección anterior, describimos brevemente del algoritmo de Lanczos, el cual permite hallar el estdo fundemental del hamiltoniano de una manera eficaz. Sin embargo, la limitación más grande del método es el gran número de estados de la base. La idea básica para superar las limitaciones del tamaño es usar una base en que el estado fundamental pueda ser representado únicamente por pocos estados. En otras palabras, encontrar un procedimiento que permita determinar o construir los estados más importantes y despreciar o descartar todos los otros tal que la pieza del espacio de Hilbert con la que uno está trabajando sea lo suficientemente pequeño. El Grupo de Renormalización de la Matriz Densidad (DMRG) es un algoritmo numérico que implementa un truncamiento eficaz del espacio de Hilbert. Este método fue desarrollado originalmente por S. White quién propuso exitosamente que el criterio para conservar y eliminar estados del espacio de Hilbert debía ser basado en la matriz densidad [62]. Desde su invención, este método ha sido utilizado para estudiar a una extensa cantidad de modelos con resultados altamente precisos, por lo que se ha constituido en un método muy utilizado actualmente en el estudio de sistemas de electrones fuertemente correlacionados de baja dimensionalidad [57, 63]. A diferencia del método de Lanczos, el DMRG puede ser implementado con alta precisión en sistemas con un máximo número de sitios $L$ de algunas centenas.

Recientemente, el DMRG ha sido extendido desde su implementación original en propiedades estáticas de temperatura cero, permitiendo el cálculo de propiedades dinámicas y termodinámicas con resultados confiables. Además, su campo de aplicación, en activo desarrollo, ha sido ampliado a otras áreas de la física más allá de la física de materia condensada [63].

El procedimiento del DMRG se puede resumir en dos etapas:

- Etapa de crecimiento.

- Etapa de barridos complementarios.

De manera general, el proceso comienza considerando un sistema de cuatro sitios como se observa en la fig. 2.8. Cada estado del sistema se expresa como una combinación de estados

$$
|\psi\rangle=\sum_{i, \alpha, \beta, j} \psi_{i, \alpha, \beta, j}|i, \alpha, \beta, j\rangle
$$

donde $i$ y $j$ son los índices de los estados de los sitios extremos izquierdo y derecho respectivamente (denominados bloques), y $\alpha$ y $\beta$ corresponden a los de los sitios centrales (denominados sitios de crecimiento). Cada bloque más el sitio central correspondiente es denominado bloque 
extendido (indicado con un recuadro de trazo punteado en la fig. 2.8). y los estados $|\psi\rangle$ de la ec. (2.94) se denominan estados de superbloque. Luego, la matriz del hamiltoniano $H$

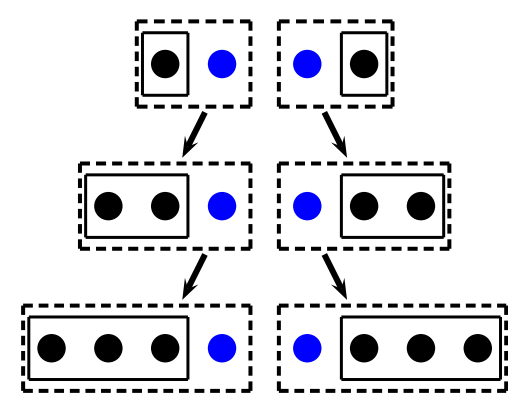

Figura 2.8: Etapa de crecimiento del método DMRG.

expresada en la base de estados del superbloque es diagonalizada (en general mediante el método de Lanczos). El estado fundamental del superbloque obtenido puede ser expresado en términos de la base de bloques extendidos como

$$
\left|\psi_{0}\right\rangle=\sum_{l m} \psi_{l m}\left|S_{l}\right\rangle \otimes\left|E_{m}\right\rangle
$$

en donde $\left|S_{l}\right\rangle$ corresponde al bloque extendido izquierdo, denominado sistema y $\left|E_{m}\right\rangle$ al derecho, denominado entorno. El proceso de truncación del espacio de Hilbert consiste en seleccionar los estados del sistema que serán conservados en la siguiente iteración en la que el tamaño total del sistema se incrementa y eliminar los estados restantes. En este truncamiento la dimensión del espacio de Hilbert del sistema se mantiene por debajo de un valor adoptado $m$. Los estados del sistema que se conservan son los que poseen mayor contribución al estado fundamental del superbloque. Esta elección se realiza utilizando la matriz densidad del sistema en el estado fundamental del superbloque que se expresa como

$$
\rho_{l l^{\prime}}=\sum_{m} \psi_{l m} \psi_{l^{\prime} m}^{*}
$$

Esta matriz densidad es diagonalizada para obtener todos sus autovalores con sus correspondientes autovectores. Los autovalores de la matriz densidad dan la probabilidad con la cual sus respectivos autovectores pueden ser encontrados en el estado fundamental. Así, los estados retenidos son aquellos de mayor autovalor en la matriz densidad. La etapa de crecimiento consiste en agregar dos nuevos sitios de crecimiento, tal como indica la figura 2.8, y conectarlos con los estados retenidos del sistema que ahora pasa a ser considerado como nuevo bloque. La matriz del hamiltoniano $H$ es entonces expresada en la base del nuevo superbloque y el proceso descrito más arriba se repite. Si bien depende del modelo hamiltoniano en particular bajo estudio y de la cantidad de estados retenidos $m$ adoptada, la truncación del espacio de Hilbert en sí comienza a realizarse luego de algunas iteraciones ya que en las iteraciones iniciales es posible retener el espacio de Hilbert completo. 
Una vez alcanzado el tamaño $L$ del sistema que se desea estudiar, es necesario complementar la etapa de crecimiento con una nueva etapa que consiste en realizar barridos complementarios para mejorar la precisión. Como hemos visto, el superbloque está compuesto por dos bloques extendidos. En el primer paso del barrido un nuevo superbloque se conforma haciendo crecer el bloque extendido izquierdo y conectándolo con el bloque extendido derecho seleccionado entre los bloques de crecimiento en orden de mantener constante el tamaño $L$ del superbloque. Este procedimiento puede ser observado en la fig. 2.9. Un nuevo superbloque es obtenido, y el proceso de selección de estados de la matriz densidad vuelve a ser realizado. Una vez que se alcanza el extremo del sistema, la dirección del barrido se invierte y ahora comienza a crecer el bloque extendido derecho, que pasa ahora a ser considerado como sistema, con entornos izquierdos calculados en el barrido anterior. Los estados que se utilizan para describir al bloque extendido que se va reduciendo se calculan refinando estados que han sido calculados en iteraciones anteriores en las que se realizó el truncamiento del bloque de crecimiento cuando poseía el mismo número de sitios, y que deben ser almacenados para tal fin.

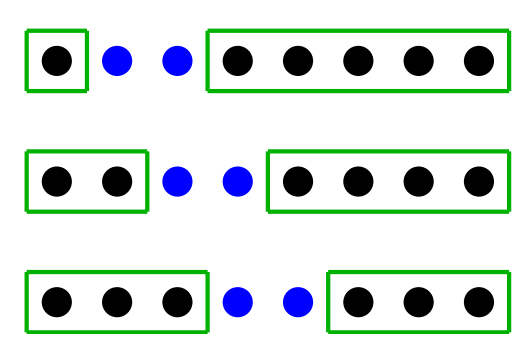

Figura 2.9: Etapa de barrido del método DMRG.

Los barridos a derecha e izquierda se pueden repetir y el proceso concluye cuando se satisface algún criterio de convergencia impuesto sobre alguna de las propiedades físicas calculadas ó el número de barridos alcanza un máximo deseado. Una característica del DMRG es que no se mantiene en una base específica del espacio de Hilbert, sino que optimiza la base que utiliza en cada iteración. Una desventaja de este proceder es que la descripción de un estado requiere la medición de observables y para el proceso de medición es necesario conocer la representación de los operadores en la base alcanzada en la iteración final. En consecuencia cada operador que uno quiere evaluar debe ser almacenado durante al cálculo, y cada vez que se cambia de base éste debe ser transformado a la nueva base.

Actualmente hay implementaciones de uso libre de estas técnicas, aplicables a modelos como los considerados en esta tesis. Entre ellos se destacan ALPS y SPINPACK.

El proyecto ALPS [64] (Algorithms and Libraries for Physics Simulations) tiene como objetivo proporcionar códigos de alto nivel para resolver modelos cuánticos de sistemas fuertemente correlacionados. SPINPACK [65] es un paquete también de acceso libre que permite calcular los autovalores y autovectores más bajos y varios valores de expectación (correlaciones de espín,... etc ) de sistemas de espines cuánticos. 


\section{Parte I}

\section{Sistemas unidimensionales acoplados con la red}





\section{Capítulo 3}

\section{Modelo de Hubbard acoplado con la red}

En este capítulo presentamos el estudio de las propiedades electrónicas de sistemas unidimensionales en el límite de baja energía por medio de bosonización abeliana. En la primera parte, hacemos una revisión de la teoría efectiva de baja energía del modelo conocido como modelo de Hubbard extendido el cual incluye interacción coulombiana entre sitios vecinos. Comentamos algunos resultados generales y conexiones con experimentos. Entonces discutimos el caso particular de sistemas con llenado 1/4 (un electrón cada dos sitios) acoplados con fonones en el límite adiabático. Dos tipos de acoplamientos considerados: tipo Holstein, que modifica la energía en el sitio, y tipo Peierls, que modula la longitud de los enlaces.

Sin las deformaciones, ambos sectores de espín y carga no poseen gap en el espectro de excitaciones a llenado 1/4. Posteriormente mostramos que la inclusión de ambos tipos de acoplamientos con la red, da como resultado una amplia variedad de fases aisladoras electroelásticas dependiendo de los valores de la relación de acoplamientos. También estudiamos el efecto de de la deformación de Peierls en la repulsión coulombiana a primeros vecinos, que puede estar presente en materiales donde la interacción de Coulomb apantallada depende fuertemente de la distancia. Discutimos la aparición de diferentes fases de ondas de carga y finalmente comparamos con algunos resultados numéricos previamente obtenidos.

\subsection{Orden de carga en conductores moleculares y de- formaciones de la red subyacente.}

Las propiedades electrónicas de sistemas de electrones en bajas dimensiones han tenido gran interés por muchas razones, la inicial es que en muchas ocasiones pueden ser utilizados para analizar el comportamiento de sistemas en dimensiones más altass. Típicamente esta conexión puede se encarada por medio de acoplar sistemas 1D para construir sistemas dimensionalmente más grandes. Por otro lado muchos materiales muestran un comportamiento unidimensional o cuasi-unidimensional en cierto rango de temperaturas donde los acopla- 


\section{Orden de carga en conductores moleculares y deformaciones de la red subyacente.}

mientos que aumentan la dimensionalidad del sistema pueden ser despreciados. Las sales de Bechgaadrs son una serie de sales orgánicas (TMTSF) ${ }_{2} \mathrm{X}$ donde el anion $\mathrm{X}$ es una molécula inorgánica monovalente $\left(\mathrm{PF}_{6}, \mathrm{AsF}_{6}, \mathrm{ClO}_{4}, \mathrm{ReO}_{4}, \mathrm{NO}_{3}, \mathrm{FSO}_{3}, \mathrm{SCN}\right.$, etc.) y TMTSF una molécula plana donora [66]. Las sales de Bechgaadrs son isoestructurales con (TMTTF $)_{2} \mathrm{X}$ conocida como sales de Fabre. Ambos (TMTSF) $)_{2} \mathrm{X}$ y (TMTTF $)_{2} \mathrm{X}$ cristalizan como paquetes de moléculas (TM=TMTSF o TMTTF) separados por los iones X (ver figura 3.1(a)).

Cálculos de primeros principios muestran que la relación de los parámetros de hopping a lo largo de la dirección de la molécula $t_{/ /}$y en la dirección perpendicular $t_{\perp}$ es del orden de $t_{\perp} / t_{/ /} \sim 0.1$ [18] permitiendo la descripción de estos compuestos en términos de modelos unidi o cuasi-unidimensionales. Además, dado que estos compuestos poseen un ión por cada dos moléculas TM la cadena efectiva se encuentra a llenado $1 / 4$.

En la figura 3.1(b) se muestra un diagrama de fases esquemático de la familia de compuestos TMTSF y TMTTF en función de la temperatura y la naturaleza de ión X o bien presión externa.

Estos compuestos presentan una gran variedad de fases como orden de carga (CO), anteferromagnetismo (AF) (sección 1.3), superconductividad (SC), espín-Peierls (SP) ${ }^{1}$, líquido de Fermi (FL) y líquido de Luttinger (LL) (ver sección 1.2.3 para estos dos últimos). A presión ambiente algunos de los compuestos $(\mathrm{TM})_{2} \mathrm{X}$, tales como $(\mathrm{TMTTF})_{2} \mathrm{PF}_{6}$ presentan fases aisladoras y mediante el incremento de la presión externa es posible llevar el sistema hacia una fase conductora. El punto principal para el estudio de estos compuestos es determinar la naturaleza del orden de carga en las fases aisladoras y esto ha sido objeto de considerables estudios tanto teóricos $[67,68,69,70,71,72,73,74]$ como experimentales $[75,76,77,78,79,80,81,82,83]$. Una gran variedad de técnicas experimentales como por ejemplo dispersión de rayos $\mathrm{X}$ muestran que la familia de compuestos TM exhiben fases donde los bonds presentan distorsiones de período dos $\left(4 \mathrm{k}_{F}\right)$ y cuatro $\left(2 \mathrm{k}_{F}\right)$.

Estos materiales involucran repulsión coulombiana tanto en el sitio como a primeros vecinos (con magnitud $U$ y $V$ respectivamente) y como dijimos presentan llenado $1 / 4$, es decir un electrón cada dos sitios de la red. Debido a la repulsión de Coulomb a primeros vecinos, es de esperar que estos materiales presenten un tipo de orden alternado similar a $\bullet-\mathrm{o}-\bullet-\mathrm{o}$, donde $\bullet$ y o corresponde a un sitio ocupado y desocupado respectivamente. Este tipo de orden corresponde al denominado Charge-Density-Wave $4 k_{F}$-CDW donde $k_{F}=\pi / 4 a$ siendo $a$ la constante de red. El nombre $4 k_{F}$ se debe a que las ondas de densidad de carga poseen un período.

Sin embargo para valores grandes de $V$, el estado fundamental corresponde a un orden $\bullet-\bullet-$ o- o $[67,68,71,72,73,74]$. Este orden de carga corresponde a una tetramerización $2 k_{F}$ CDW y como veremos puede coexistir ya sea con una modulación periódica $2 k_{F}$ de la distancia entre sitios, llamada Bond-Ordered-Wave (BOW) o con una mezcla de ordenes de carga $2 k_{F}+4 k_{F}$ BOW. Este orden de carga es llamado en la literatura Bond-Charge Density Wave (BCDW).

\footnotetext{
${ }^{1}$ La fase de espín-Peierls se establece a partir de una transición magneto-elástica en materiales antiferromagnéticos cuando la energía magnética decrece debido a la fomración de pares de singletes de espín produciendo como resultado la dimerización del arreglo o red regular. En el capítulo 4 es dedicado al estudio de estas faseses.
} 


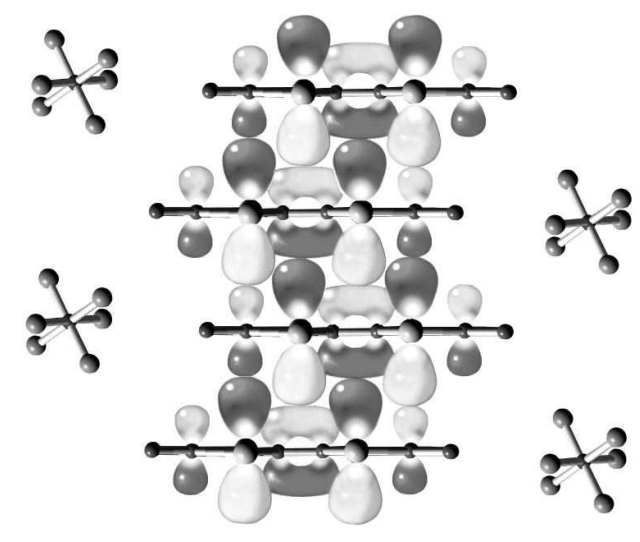

(a)

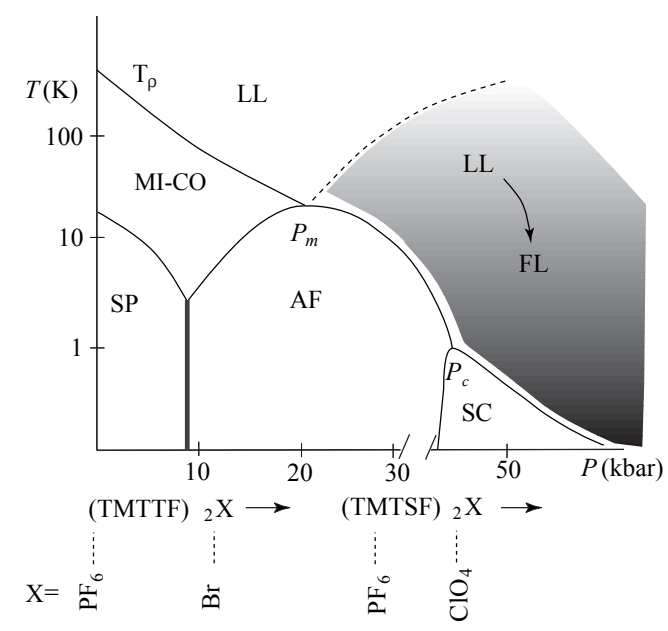

(b)

Figura 3.1: (a) Estructura de las sales de Bechgaadrs y Fabre. El arreglo plano de las moléculas orgánicas TM con sus orbitales es representada entre pequeñas aniones. (b) Diagrama de fases genérico de las sales de Bechgaard/Fabre como función de la temperatura y presión $(\sim 1 / U)$ o sustitución de $\mathrm{X}$ de aniones. Las distintas fases observadas son: LL: líquido de Luttinger, MI: aislador de Mott, CO: orden de carga, SP: espín-Peierls, AF: antiferromagnetismo, SC: superconductividad, FL: líquido de Fermi.

Teniendo en cuenta que estos sistemas pueden presentar una modulación geométrica en los bonds, es de esperar que si la interacción coulombiana efectiva entre dos sitios $i$ y $j$ es exponencialmente de corto alcance, o sea de la forma

$$
\frac{\exp [-\alpha|i-j|]}{|i-j|^{\xi}} \text { con } \alpha, \xi>0
$$

las distorsiones de la red pueden tener un efecto importante en la modulación de las constantes de acoplamiento. Un análisis profundo del diagrama de fases 3.1 y las diferentes fases presentes está más allá del alcance del presente manuscrito y para una revisón de los aspectos teóricos y experimentales generales de las fases aisladoras en compuestos moleculares ver por ejemplo [84].

Motivados por la discución anterior, en este capítulo estudiamos, mediante bosonización abeliana (sección 2.1) y una análisis semiclásico de la teoría efectiva, el diagrama de fases del estado fundamental del modelo de Hubbard sometido a dos tipos de interacción electrónfonón en el límite adiabático (ver sección 1.4): el acoplamiento de Peierls en el término de hopping y el de Holstein en la densidad local de carga. La inclusión de ambos acoplamientos en el hamiltoniano de Hubbard define lo que se conoce como el modelo de Peierls-HolsteinHubbard. Además de estos dos términos, incluimos el efecto de la deformación de Peierls en el término de repulsión coulombiana a primeros vecinos el cual puede producir un cambio importante en las propiedades electrónicas de sistemas cuya interacción coulombiana es de la forma (3.1). Mostramos que el estado fundamental a temperatura cero presenta varios 
patrones de deformaciones de período dos y cuatro que rompen espontáneamente la simetría de traslación del sistema, dependiendo de la magnitud de los parámetros microscópicos del modelo (ver figura 3.2). Cuando el efecto de la deformación de los bonds es tenido en cuenta en el término de repulsión coulombiana, el diagrama de fases presenta cambios importantes indicando que este término debe ser considerado a la hora de contrastar con resultados experimentales.

\subsection{Hamiltoniano de Hubbard extendido}

En esta sección presentamos el modelo de Hubbard extendido acoplado con fonones adiabáticos, y su tratamiento analítico. En tal límite, el sector de los fonones es descrito por deformaciones clásicas y analizamos los posibles patrones.

Consideremos el modelo de Hubbard extendido con hamiltoniano dado por:

$$
\begin{aligned}
\mathcal{H}= & -\sum_{j, \sigma} t_{j, j+1}\left[c_{j, \sigma}^{\dagger} c_{j+1, \sigma}+c_{j+1, \sigma}^{\dagger} c_{j, \sigma}\right] \\
& +U \sum_{j} n_{j, \uparrow} n_{j, \downarrow}+\sum_{j} V_{j, j+1} n_{j} n_{j+1} \\
& +\sum_{j} \beta_{j}\left(n_{j}-\frac{1}{2}\right)
\end{aligned}
$$

donde $c_{j, \sigma}$ denota el operador de aniquilación de un electrón con espín $\sigma(=\uparrow, \downarrow)$ en el $j$-ésimo sitio, y $n_{j}=n_{j, \uparrow}+n_{j, \downarrow}$ es la densidad de carga con $n_{j, \sigma}=c_{j, \sigma}^{\dagger} c_{j, \sigma} \cdot t_{i, i+1}$ es la integral de hopping, los parámetros $U(>0)$ y $V_{j, j+1}(>0)$ denotan las magnitudes de la interacción coulombiana efectiva en el sitio y a primeros vecinos respectivamente; mientras que $\beta_{j}$ representa la deformación molecular interna. Como mencionamos antes, nos enfocamos en el caso de llenado $1 / 4$ que corresponde una densidad promedio $\langle n\rangle=1 / 2$.

La interacción del sector electrónico con los fonones en el límite adiabático (ver sección 1.4) se obtiene por medio de una expansión lineal de $t_{j, j+1}$ alrededor de $t$,

$$
t_{j, j+1} \simeq t\left(1-g_{P} \delta_{P, j}\right)
$$

donde $\delta_{P, j}$ mide la distorsión de la constante de red entre los sitios $j, j+1$ y $g_{P}$ mide la interacción electrón-fonón correspondiente al acoplamiento de Peierls.

Por otro lado, las vibraciones de las moléculas producen cambios locales en la energía y pueden ser tenidos en cuenta por el término de Holstein (ver sección 1.4)

$$
g_{H} \sum_{j} \delta_{H, j}\left(n_{j}-\frac{1}{2}\right),
$$

donde $g_{H}$ es la constante de acoplamiento y $\delta_{H, j}$ mide la deformación molecular interna en el sitio $j$ (en la ecuación 3.2 realizamos el cambio $\beta_{j} \rightarrow g_{H} \delta_{H, j}$ ). 
Además de los términos de interacción antes descritos, incluimos el efecto de la llamada deformación de Peierls en el término de la repulsión coulombiana a primeros vecinos $V$ que puede ser de real importancia cuando el decaimiento es exponencialmente decreciente de la forma (3.1). En ese caso, una pequeña variación en la distancia produce un cambio importante en la interacción. Más específicamente, realizamos una expansión lineal de los acoplamientos $V_{j, j+1}$ alrededor del valor homogéneo $V$,

$$
V_{j, j+1} \simeq V\left(1-g_{V} \delta_{P, j}\right)
$$

donde $g_{V}$ es la constante de acoplamiento que mide el efecto de la deformación de los enlaces sobre la repulsión de Coulomb. Finalmente, el hamiltoniano completo, incluyendo la energía elástica en la aproximación adiabática está dado por:

$$
\begin{aligned}
\mathcal{H}= & -t \sum_{j, \sigma}\left(1-g_{P} \delta_{P, j}\right)\left[c_{j, \sigma}^{\dagger} c_{j+1, \sigma}+c_{j+1, \sigma}^{\dagger} c_{j, \sigma}\right] \\
& +U \sum_{j} n_{j, \uparrow} n_{j, \downarrow}+V \sum_{j}\left(1-g_{V} \delta_{P, j}\right) n_{j} n_{j+1} \\
& +g_{H} \sum_{j} \delta_{H, j}\left(n_{j}-\frac{1}{2}\right) \\
& +\frac{K_{P}}{2} \sum_{j} \delta_{P, j}^{2}+\frac{K_{H}}{2} \sum_{j} \delta_{H, j}^{2}
\end{aligned}
$$

donde los parámetros $K_{H}$ y $K_{P}$ son las constantes elásticas para las deformaciones tipo Holstein y Peierls respectivamente.

Como mencionamos en la introducción del capítulo (sección 3.1), se han llevado a cabo varios estudios numéricos (diagonalización exacta, DMRG) de sistemas similares al propuesto en este trabajo. En esta parte vamos a realizar un breve resumen de los resultados obtenidos por Clay et al [85] mediante diagonalización exacta del modelo (3.4) sin acoplamiento de las deformaciones de Peierls en la repulsión coulombiana a primeros vecinos, i.e. $g_{V}=0$.

Este trabajo fué realizado por medio de diagonalización exacta de cadenas de hasta 16 sitios con condiciones de contorno periódicas con un procedimiento autoconsistente para determinar el patrón de las deformaciones del estado fundamental. El método consiste en minimizar la energía total $E\left(\delta_{i}\right)$ respecto del conjunto de distorsiones $\delta_{i}$ resolviendo las siguientes ecuaciones acopladas de manera iterativa

$$
\begin{aligned}
K_{P} \delta_{P, j}+t g_{P} \sum_{\sigma}\left\langle c_{j, \sigma}^{\dagger} c_{j+1, \sigma}+c_{j+1, \sigma}^{\dagger} c_{j, \sigma}\right\rangle & =0 \\
K_{H} \delta_{H, j}+g_{H}\left\langle n_{j}-\frac{1}{2}\right\rangle & =0
\end{aligned}
$$

donde $\langle\ldots\rangle$ es el valor medio obtenido en el estado fundamental electrónico.

En la figura 3.3 se muestra el diagrama de fases del estado fundamental en el plano $\lambda_{\alpha}-\lambda_{\beta}{ }^{2}$

\footnotetext{
${ }^{2} \lambda_{\alpha}=g_{P}^{2} / t K_{P}$ y $\lambda_{\beta}=g_{H}^{2} / t K_{H}$
} 


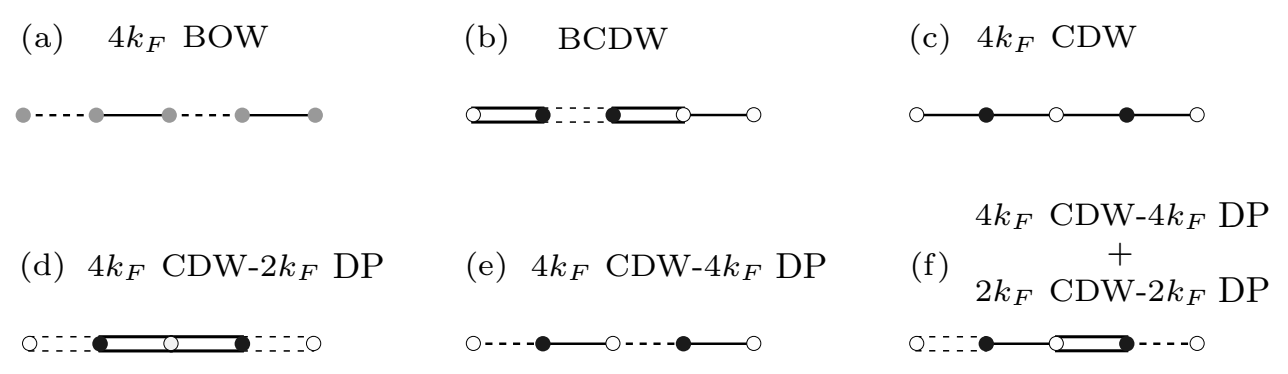

Figura 3.2: Deformaciones de la red y estados con orden de densidad de carga del modelo (3.4) de a llenado $1 / 4$. Los círculos en gris, negro y blanco corresponden a sitios con carga $\left\langle n_{i}\right\rangle=0.5,1$ y 0 , respectivamente. El patrón (a) representa el estado $4 k_{F}$ BOW con enlaces dimerizados y densidad de carga uniforme, (b) representa el estado BCDW, con orden de enlaces $2 k_{F}>4 k_{F}$ y orden de carga $\bullet-\bullet-$ o- o. (c) corresponde a un orden de carga $4 k_{F}$ CDW con $\bullet-$ o $-\bullet-$ o y enlaces uniformes. (d) muestra el estado $4 k_{F}$ CDW- $2 k_{F}$ PD, con orden de carga $\bullet-$ o $-\bullet-$ o y orden de los enlaces $2 k_{F}$. Para $g_{V}=0.2$, en (e) tenemos un orden de carga i.e. $4 k_{F} \mathrm{CDW}-4 k_{F} \mathrm{PD}$ con $\bullet-\mathrm{o}-\bullet-$ o junto con una dimerización en los enlaces. Finalmente, la fase (f) denotada por $\left(4 k_{F} \mathrm{CDW}-4 k_{F} \mathrm{PD}+2 k_{F} \mathrm{CDW}-2 k_{F} \mathrm{PD}\right)$ presenta una mezcla de ordenes $2 k_{F}$ y $4 k_{F}$ tanto en la densidad de carga como en los enlaces.

para una cadena periódica de 16 sitios. En primer lugar, podemos observar que para valores pequeños de $\lambda_{\alpha}, \lambda_{\beta}$, el sistema no se deforma; esto es una consecuencia del tamaño del sistema y dicha región disminuye a medida que se incrementa el valor de $V$.

Para valores grandes de $\lambda_{\alpha}$ (mucho mayores que $\lambda_{\beta}$ ) el sistema presenta un orden de carga similar al mostrado en la figura 3.2(b) (BCDW); para $\lambda_{\alpha} \ll \lambda_{\beta}$ el estado fundamental corresponde al $4 k_{F}$ CDW mostrado en la figura 3.2(c).

En la siguiente sección vamos a derivar un hamiltoniano efectivo para el modelo (3.4) por medio de bosonización y analizaremos las fases posibles del estado fundamental incluyendo la interacción $g_{V}>0$.

\subsection{Bosonización y análisis semiclásico}

En esta sección analizamos las propiedades de baja energía del modelo (3.4) utilizando el método de bosonización abeliana descrito en la sección 2.1.

En el apéndice A mostramos que, dentro del lenguaje de bosonización, las deformaciones de período cuatro son las únicas que causan conmensurabilidad de perturbaciones relevantes a llenado $\langle n\rangle=1 / 2$ y proveen un mecanismo para la formación de un gap en el sector de carga [86]. De esta manera el método de bosonización selecciona patrones de deformación para las cantidades $\delta_{a, j}(a=P, H$ y $j=1, \ldots, N)$ en la ecuación (3.4). Estos resultados han sido confirmados mediante métodos numéricos autoconsistentes [85] revelando que las deformaciones de período cuatro predominan por sobre el resto.

A llenado 1/4, la deformación más general de período cuatro (sin desplazamientos colectivos) 

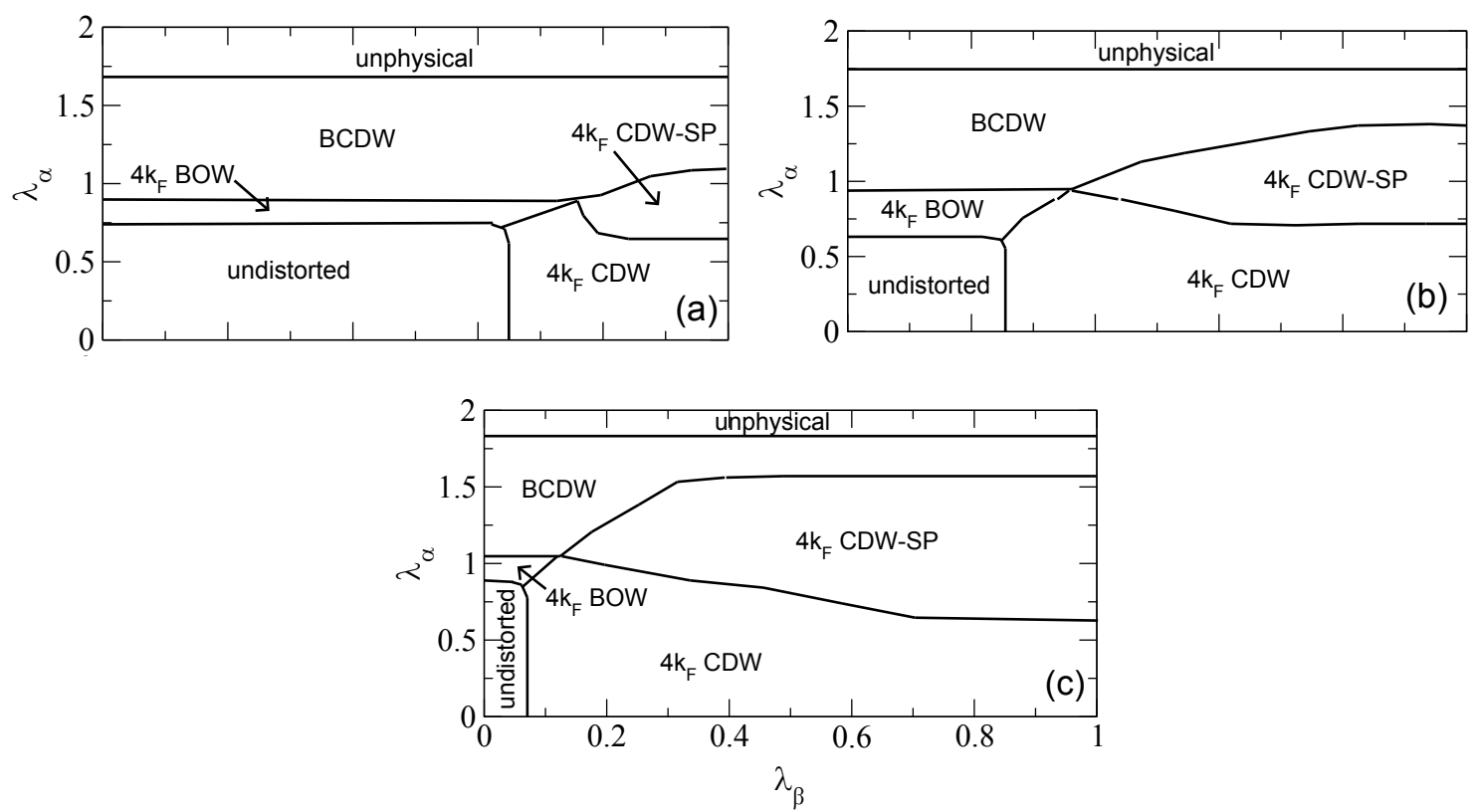

Figura 3.3: Diagrama de fases del modelo (3.4) en el plano $\lambda_{\alpha}-\lambda_{\beta}$ para $U=8 t$ y (a) $V=2 t$; (b) $V=3 t$; y (c) $V=4 t$ para una cadena periódica de 16 sitios.

que selecciona bosonización puede ser parametrizada como

$$
\begin{aligned}
\delta_{P, j} & =\delta_{P, d} \cos \left(\frac{\pi}{a} x_{j}\right)+\delta_{P, t} \cos \left(\frac{\pi}{2 a} x_{j}+\xi-\frac{\pi}{4}\right) \\
\delta_{H, j} & =\delta_{H, d} \cos \left(\frac{\pi}{a} x_{j}\right)+\delta_{H, t} \cos \left(\frac{\pi}{2 a} x_{j}-\zeta\right)
\end{aligned}
$$

donde $x_{j}=j a$ es la posición del $j$-ésimo sitio, $a$ es el espaciamiento de la red que por simplicidad vamos a fijar a 1 en el resto del capítulo. Las fases $\xi$ y $\zeta$ determinarán los patrones espaciales de la tetramerización $\delta_{P, t} \mathrm{y} \delta_{H, t}$ respectivamente, mientras que $\delta_{P, d} \mathrm{y} \delta_{H, d}$ son las amplitudes de dimerización de la red y en el sitio. La ecuación (3.7) define la parametrización más general que selecciona bosonización. Para facilitar el análisis adimensionalizamos los parámetros usando $t$ como escala de energía como sigue:

$$
\begin{aligned}
g_{P} \rightarrow \tilde{g}_{P} & =\left(t / K_{P}\right)^{1 / 2} g_{P}, \quad \delta_{P, s} \rightarrow \tilde{\delta}_{P, s}=\left(K_{P} / t\right)^{1 / 2} \delta_{P, s} \\
g_{H} \rightarrow \tilde{g}_{H} & =\left(t / K_{H}\right)^{1 / 2} g_{H}, \quad \delta_{H, s} \rightarrow \tilde{\delta}_{H, s}=\left(K_{H} / t\right)^{1 / 2} \delta_{H, s} \\
g_{V} \rightarrow \tilde{g}_{V} & =\left(t / K_{P}\right)^{1 / 2} g_{V},
\end{aligned}
$$

con $s=d, t$.

Para $U, V \neq 0$, la teoría de baja energía correspondiente a (3.4) escrita en términos de los campos bosónicos $\phi_{R, L, \sigma}$ tiene un forma complicada, mezclando las componentes $\uparrow \mathrm{y} \downarrow$. Sin embargo, introduciendo los campos $\varphi_{\sigma}=\phi_{R, \sigma}+\phi_{L, \sigma}$ (ver sección 2.1.3) y las combinaciones lineales de $\varphi_{\uparrow} \mathrm{y} \varphi_{\downarrow}$ para describir el sector de carga y espín,

$$
\varphi_{c}=\frac{1}{\sqrt{2}}\left(\varphi_{\uparrow}+\varphi_{\downarrow}\right), \quad \varphi_{s}=\frac{1}{\sqrt{2}}\left(\varphi_{\uparrow}-\varphi_{\downarrow}\right)
$$




\section{Bosonización y análisis semiclásico}

podemos reescribir $\mathcal{H}=\int_{0}^{L} d x h$ desacoplando ambos sectores excepto en un término de interacción entre ellos como

$$
h=h_{\text {elástico }}+h_{\text {libre }}+\mathcal{V}_{\text {efectivo }}
$$

donde $h_{\text {elástico }}$ es la densidad de energía elástica correspondiente a las deformaciones de Peierls y Holstein

$$
h_{\text {elástico }}=\frac{1}{2}\left(\frac{1}{2} \tilde{\delta}_{P, t}^{2}+\tilde{\delta}_{P, d}^{2}\right)+\frac{1}{2}\left(\frac{1}{2} \tilde{\delta}_{H, t}^{2}+\tilde{\delta}_{H, d}^{2}\right),
$$

$h_{\text {libre }}$ contiene la parte libre o gaussiana correspondiente a los sectores de carga y espín

$$
h_{\text {libre }}=\frac{v_{c}}{2}\left[\frac{1}{K_{c}}\left(\partial_{x} \varphi_{c}\right)^{2}+K_{c}\left(\partial_{x} \theta_{c}\right)^{2}\right]+\frac{v_{s}}{2}\left[\frac{1}{K_{s}}\left(\partial_{x} \varphi_{s}\right)^{2}+K_{s}\left(\partial_{x} \theta_{s}\right)^{2}\right]
$$

donde los parámetros $v_{c}$ y $v_{s}$ son las velocidades de las excitaciones de carga y espín, y $K_{c}$ y $K_{s}$ son los correspondientes parámetros de Luttinger. Finalmente el potencial de interacción llamado $\mathcal{V}_{\text {efectivo }}$ incluye tres términos

$$
\mathcal{V}_{\text {efectivo }}=\mathcal{V}_{c}+\mathcal{V}_{s}+\mathcal{V}_{\text {int }}
$$

los primeros dos corresponden a la autointeracción en los sectores de carga y espín,

$$
\begin{aligned}
\mathcal{V}_{c}= & \tilde{g}_{1 / 4} \cos 4 \sqrt{2 \pi} \varphi_{c}-\lambda_{1} \tilde{g}_{P} \tilde{\delta}_{P, d} \sin 2 \sqrt{2 \pi} \varphi_{c}+\lambda_{2} \tilde{g}_{H} \tilde{\delta}_{H, d} \cos 2 \sqrt{2 \pi} \varphi_{c} \\
& +\lambda_{3} \tilde{g}_{V} \tilde{\delta}_{P, d} \cos 2 \sqrt{2 \pi} \varphi_{c} \\
\mathcal{V}_{\sigma}= & \tilde{g}_{s} \cos 2 \sqrt{2 \pi} \varphi_{s}
\end{aligned}
$$

mientras que el tercero mezcla ambos sectores,

$$
\begin{aligned}
\mathcal{V}_{i n t}= & -\tilde{g}_{P} \tilde{\delta}_{P, t} \cos \left(\sqrt{2 \pi} \varphi_{c}+\xi\right) \cos \sqrt{2 \pi} \varphi_{s}-\tilde{g}_{H} \tilde{\delta}_{H, t} \sin \left(\sqrt{2 \pi} \varphi_{c}+\zeta\right) \cos \sqrt{2 \pi} \varphi_{s} \\
& -\lambda_{4} \tilde{g}_{V} \tilde{\delta}_{P, d} \cos 2 \sqrt{2 \pi} \varphi_{c} \cos 2 \sqrt{2 \pi} \varphi_{s}+\lambda_{5} \tilde{g}_{V} \tilde{\delta}_{P, t} \cos \sqrt{2 \pi} \varphi_{s} \sin \left(3 \sqrt{2 \pi} \varphi_{c}+\xi-\frac{\pi}{4}\right) \\
& -\lambda_{6} \tilde{g}_{V} \tilde{\delta}_{P, t} \cos \sqrt{2 \pi} \varphi_{s} \sin \left(\sqrt{2 \pi} \varphi_{c}-\xi+\frac{\pi}{4}\right)
\end{aligned}
$$

En las ecuaciones (3.12)-(3.15) $\varphi_{c}$ y $\varphi_{s}$ son los campos bosónicos correspondientes al sector de carga y espín respectivamente, $\theta_{c, s}$ son los campos duales definidos por $\partial_{x} \theta_{c, s}=\partial_{t} \varphi_{c, s} \mathrm{y}$ las fases $\xi$ y $\zeta$ a calcular determinan el patrón espacial de la tetramerización (ver ecuación $(3.7))$.

Como mostramos en el apéndice A, los parámetros de Luttinger determinan las dimensiones de escala de los operadores que aparecen en la ecuación (3.15). Realizando un análisis similar se puede mostrar que: 


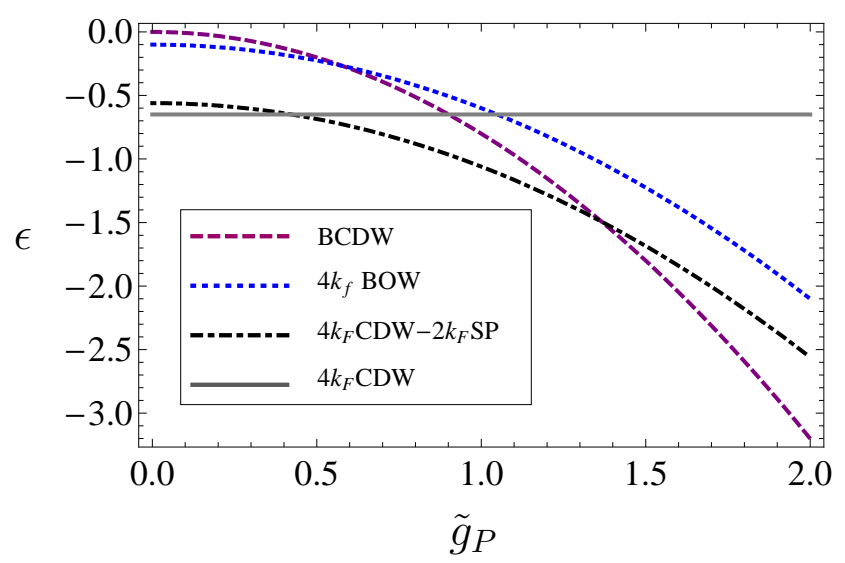

Figura 3.4: Energías mínimas de cada fase electro-elástica en términos de $\tilde{g}_{P}$ para valores fijos de $\tilde{g}_{1 / 4}=-1.5, \tilde{g}_{H}=2.2$ y $\tilde{g}_{V}=0$.

- En el término $\mathcal{V}_{c}$, las dimensiones de escala están respectivamente dadas por $8 K_{c}, 2 K_{c}$, $2 K_{c}$ y $2 K_{c}$.

- En el término $\mathcal{V}_{s}$ la dimensión es $2 K_{s}$.

- Finalmente en el término $\mathcal{V}_{\text {int }}$ las dimensiones de cada término son $K_{c} / 2+K_{s} / 2$, $K_{c} / 2+K_{s} / 2,2 K_{c}+2 K_{s}, K_{s} / 2+9 K_{c} / 2$ y $K_{c} / 2+K_{s} / 2$ respectivamente.

Todos estos operadores son relevantes (esto es, la dimensión de escala es menor que 2) si $K_{c}<1 / 4$ y $K_{c}+K_{s}<1$.

Las constantes de acoplamientos efectivas en las ecuaciones (3.14) y (3.15) se relacionan con lo parámetros microscópicos por

$$
\begin{array}{ll}
\tilde{g}_{1 / 4} \propto U^{2}(U-4 V) & \lambda_{3} \propto\left(U-D_{2}\right) V \\
\tilde{g}_{s} \propto U\left(1-4 D_{1}(U / 2-V)\right) & \lambda_{4} \propto V \\
\lambda_{1} \propto U & \lambda_{5} \propto U V \\
\lambda_{2} \propto U & \lambda_{6} \propto\left(U-D_{3}\right) V
\end{array}
$$

donde $D_{1} \sim 1, D_{2}=8$ y $D_{3}=\pi^{2} / 8$.

El potencial $\mathcal{V}_{\text {efectivo }}$ en la ecuación (3.15), define una teoría multi-seno-Gordon caracterizada por la presencia de varios términos cosenos con diferentes frecuencias. Este tipo de toerías han sido estudiadas anteriormente [87, 88, 89, 90, 91, 92] enfocándose principalmente la competencia entre los diferentes armónicos.

En nuestro estudio realizamos un análisis semiclásico de la teoría efectiva considerando configuraciones estáticas de los campos bosónicos, permitiéndonos obtener un diagrama de fases cualitativo del estado fundamental. Un tratamiento más completo podría incluir fluctuaciones cuánticas para introducir correcciones a los bordes de las fases obtenidas semiclásicamente. Sin embargo este tratamiento escapa del presente análisis y puede quedar como futuro trabajo. 
Para determinar los diferentes patrones de carga utilizamos el operador densidad que puede ser representado en el lenguaje de bosonización como (ver apéndice A)

$$
\begin{aligned}
n(x)= & \psi_{\uparrow}^{\dagger}(x) \psi_{\uparrow}(x)+\psi_{\downarrow}^{\dagger}(x) \psi_{\downarrow}(x) \\
= & \frac{1}{2}+\sqrt{\frac{2}{\pi}} \frac{d \varphi_{c}(x)}{d x}-C \sin \left[2 k_{F} x+\sqrt{2 \pi} \varphi_{c}(x)\right] \cos \left[\sqrt{2 \pi} \varphi_{s}(x)\right] \\
& -C^{\prime} \cos \left[4 k_{F} x+2 \sqrt{2 \pi} \varphi_{c}(x)\right]
\end{aligned}
$$

donde $C$ y $C^{\prime}$ son constantes no-universales y el momento de Fermi toma el valor de $k_{F}=\pi / 4$ a llenado $1 / 4$.

Usamos los coeficientes obtenidos perturbativamente $C, C^{\prime}$ y asumimos la dependencia de las constantes de acoplamientos $\tilde{g}_{1 / 4}, \tilde{g}_{s} \mathrm{y}$ los $\lambda$ 's en términos de los parámetros microscópicos mostrada en las ecuaciones (3.16).

El análisis de la teoría efectiva definida por las ecuaciones (3.10)-(3.15) consiste en tratar los términos de autointeracción (3.15) como un potencial clásico a ser evaluado configuraciones constantes de los campos $\varphi_{s, c}$ correspondiendo al estado fundamental. Dentro de esta aproximación la parte libre (3.12) puede ser descartada y la energía que se obtiene es

$$
\epsilon=\mathcal{H}_{\text {elástico }}+\mathcal{V}_{\text {eff }}\left(\tilde{\delta}_{P, d}, \tilde{\delta}_{P, t}, \tilde{\delta}_{H, d}, \tilde{\delta}_{H, t}, \varphi_{c}, \varphi_{s}, \xi, \zeta\right)
$$

Este tipo de análisis ha mostrado ser muy efectivo a la hora de determinar fases ordenadas tanto de sistemas electrónicos como de espines [46, 91, 92, 93].

El objetivo es entonces buscar las posibles deformaciones que minimizan la energía definida por la ecuación (3.18) con respecto al caso no deformado $\left(\tilde{\delta}_{P, d}=\tilde{\delta}_{P, t}=\tilde{\delta}_{H, d}=\tilde{\delta}_{H, t}=0\right)$. Para ello debemos resolver el conjunto de ecuaciones acopladas

$$
\begin{aligned}
& \frac{\tilde{\delta}_{a, t}}{2}+\frac{\partial \mathcal{V}_{\text {efectivo }}}{\partial \delta_{a, t}}=0 \\
& \tilde{\delta}_{a, t}+\frac{\partial \mathcal{V}_{\text {efectivo }}}{\partial \delta_{a, d}}=0 \\
& \frac{\partial \mathcal{V}_{\text {efectivo }}}{\partial \xi}=0 \\
& \frac{\partial \mathcal{V}_{\text {efectivo }}}{\partial \zeta}=0 \\
& \frac{\partial \mathcal{V}_{\text {efectivo }}}{\partial \varphi_{b}}=0
\end{aligned}
$$

donde $a=P, H$ y $b=c, s$.

Dependiendo de los coeficientes $\tilde{g}_{1 / 4}, \tilde{g}_{s}$ y $\lambda$ 's que a su vez dependen de los parámetros microscópicos del modelo, uno de los puntos críticos determinados por las ecuaciones (3.19)(3.24) es seleccionado como el mínimo global y determina la fase electro-elástica del estado fundamental. Para ilustrar el análisis realizado, en la figura 3.4 se muestra la evolución de 
las energías como función del acoplamiento tipo Peierls $\tilde{g}_{P}$ para $\tilde{g}_{1 / 4}=-1.5, \tilde{g}_{H}=2.2$ y $\tilde{g}_{V}=0$. El cruce de las diferentes soluciones determina el borde de las distintas fases. Los resultados obtenidos son resumidos en la figura (3.5). Para facilitar la notación realizamos un escaleo de los campos bosónicos $\left(\phi_{c}, \phi_{s}\right) \rightarrow \frac{1}{\sqrt{2 \pi}}\left(\phi_{c}, \phi_{s}\right)$ en la ecuación (3.15).

\subsection{Resultados}

Para comenzar, analicemos con el caso $g_{V}=0$, que corresponde a no incluir el efecto de la deformación de Peierls en la repulsión coulombiana a primeros vecinos. Para empezar notemos que, sin la inclusión de las deformaciones de Peierls y Holstein, de la expresión (3.16) se desprende que $\tilde{g}_{1 / 4}$ es positivo para $V$ pequeño y se vuelve negativo $V$ suficientemente grande. En ese caso las soluciones de mínima energía arrojan

- $\phi_{c}=\pi / 4$, para $\tilde{g}_{1 / 4}>0$

- $\phi_{c}=0$, para $\tilde{g}_{1 / 4}<0$.

Ahora veamos el efecto de los acoplamientos $\tilde{g}_{P}$ y $\tilde{g}_{H}$. En la figura 3.5(a) fijamos $\tilde{g}_{1 / 4}>0$ (o $U>4 V$ ), y aumentamos $\tilde{g}_{P}$ desde cero (para un valor fijo y pequeño de $\tilde{g}_{H}$ ).

En este caso la solución corresponde a

- $4 \mathrm{k}_{F} \mathrm{BOW}$ :

$\left(\phi_{c}, \phi_{s}\right)=\left(\frac{\pi}{4}, \frac{\pi}{2}\right)$, (independientemente de $\left.\xi, \zeta\right)$,

En este caso los enlaces presentan un patrón de período cuatro $\left(4 \mathrm{k}_{F}\right)$ mientras que la densidad de carga en cada sitio permanece uniforme $\left(\left\langle n_{i}\right\rangle=1 / 2\right)$. Esta fase es esquematizada en la figura 3.2(a).

Cuando $\tilde{g}_{P}$ supera un valor crítico $\tilde{g}_{P} \gtrsim \tilde{g}_{P}^{c}\left(\tilde{g}_{H}\right)$ el estado fundamental corresponde a la fase BCDW, obteniendo

- BCDW:

$\left(\xi, \zeta, \phi_{c}, \phi_{s}\right)=\left(\frac{\pi}{4}, \frac{\pi}{4}, \frac{\pi}{4}, \pi\right)$

el estado fundamental adquiere un orden de carga es de la forma $\mathbf{o}-\boldsymbol{\bullet}-\bullet-$ o, mientras que, los enlaces presentan un patrón de la forma $t\left(1+\alpha_{1}\right), t\left(1-\alpha_{2}\right), t\left(1+\alpha_{1}\right), t\left(1+\alpha_{3}\right)$ $\left(\operatorname{con} \alpha_{i}>0\right)$.

Ahora consideremos la situación cuando $\tilde{g}_{H}$ supera un valor crítico $\tilde{g}_{H}^{c}\left(\tilde{g}_{P}\right)$. En este caso la solución selecciona

- $4 k_{F} \mathrm{CDW}$ : $\left(\phi_{c}, \phi_{s}\right)=\left(0, \frac{\pi}{2}\right)$ (independiente de $\left.\xi, \zeta\right)$

con orden de carga $\bullet-$ o - $\bullet-$ o y enlaces uniformes (figura 3.2(c). La fase $4 k_{F}$ CDW-2 $k_{F}$ $\mathrm{PD}$ en donde $2 k_{F}$ PD denota una deformacion de Peierls de período cuatro, es mostrada en la figura $3.2(\mathrm{~d})$ y ocurre únicamente para valores grandes de $\tilde{g}_{H}$ y $\tilde{g}_{P}$ seleccionando 
- $4 k_{F} \mathrm{CDW}-2 k_{F} \mathrm{PD}$ :

$\left(\xi, \zeta, \phi_{c}, \phi_{s}\right)=\left(\frac{\pi}{2}, \pi, 0, \pi\right)$

En la figura 3.5 podemos observar el cambio que se produce cuando el acoplamiento efectivo $\tilde{g}_{1 / 4}<0$ (o bien $V>U / 4$ ). Primero, el ancho de la región $4 k_{F}$ BOW disminuye considerablemente mostrando que cuando la interacción electrón-fonón es suficientemente grande, la tendencia "natural" para el orden de carga es $\bullet-$ o $-\bullet-$ o, para $\tilde{g}_{P}$ pequeño. Por la misma razón, los anchos de las regiones $4 k_{F}$ CDW y $4 k_{F}$ CDW- $2 k_{F}$ PD se incrementan considerablemente cuando $\tilde{g}_{1 / 4}$ es negativo. Hasta acá se puede observar que las fases encontradas coinciden con las obtenidas mediante diagonalización exacta de sistemas pequeños mencionadas en la sección anterior. Además los diagramas de fases para ambos métodos muestran las mismas regiones al menos cualitativamente sustentando nuestra aproximación.

Finalmente analicemos el efecto de la deformación de Peierls en la interacción coulombiana a partir del acoplamiento $\tilde{g}_{V}$. Notemos que el efecto de este término es más importante para valores grandes de la interacción $V$. El resultado que se obtiene es que es que las fases que presentan orden de carga $4 \mathrm{k}_{F}$ dominan el diagrama de fases por sobre las fases sin orden o con orden $2 \mathrm{k}_{F}$. Por ejemplo, para $\tilde{g}_{V}=0.2$ y $\tilde{g}_{1 / 4}<0$ se puede observar que la región $4 k_{F}$ BOW "desaparece" y para $\tilde{g}_{P} \lesssim 0.5$ la solución selecciona

- $4 k_{F} \mathrm{CDW}-4 k_{F} \mathrm{PD}$ :

$\left(\xi, \zeta, \phi_{c}, \phi_{s}\right)=\left(f_{1}, \frac{\pi}{2}, 0, \pi\right)$

donde $f_{1}$ es una función de los multiples acoplamientos del modelo demasiado extensa como para presentarla aquí. La diferencia más grande es que aparece una pequeña dimerización $4 k_{F}$ en los bonds (ver figura $3.2(\mathrm{e})$ ). Por otro lado para $0.5 \lesssim \tilde{g}_{P} \lesssim 1.5$ la solución arroja

- $4 k_{F} \mathrm{CDW}-4 k_{F} \mathrm{PD}+2 k_{F} \mathrm{CDW}-2 k_{F} \mathrm{PD}$ : $\left(\xi, \zeta, \phi_{c}, \phi_{s}\right)=\left(f_{2}, \frac{\pi}{2}, 0, \pi\right)$.

donde nuevamente $f_{2}$ es una función de los multiples acoplamientos demasiado extensa como para presentarla aquí.En esta última solución se observa una mezcla de ordenes de carga y enlaces $4 k_{F}$ y $2 k_{F}$ con la amplitud $4 k_{F}$ mayor que la $2 k_{F}$ (ver figura $3.2(\mathrm{f})$ ).

\subsection{Conclusiones}

El modelo de Hubbard extendido a llenado 1/4 es un buen candidato para describir materiales orgánicos como las sales de Bechgaard y sales de Fabre. Estos sistemas exhiben diagramas de fases muy interesantes, incluyendo varios tipos de ordenes de carga. En este capítulo hemos investigado el diagrama de fases del estado fundamental de del modelo de Hubbard extendido a llenado $1 / 4(n=1 / 2)$ acoplado a fonones en la aproximación adiabática que incluye interacciones tipo Holstein y Peierls. Además de los términos convencionales, hemos incluido los efectos de la deformación de Peierls en el término de interacción coulombiana efectiva a primeros vecinos. Este último término tiene efectos importantes en 

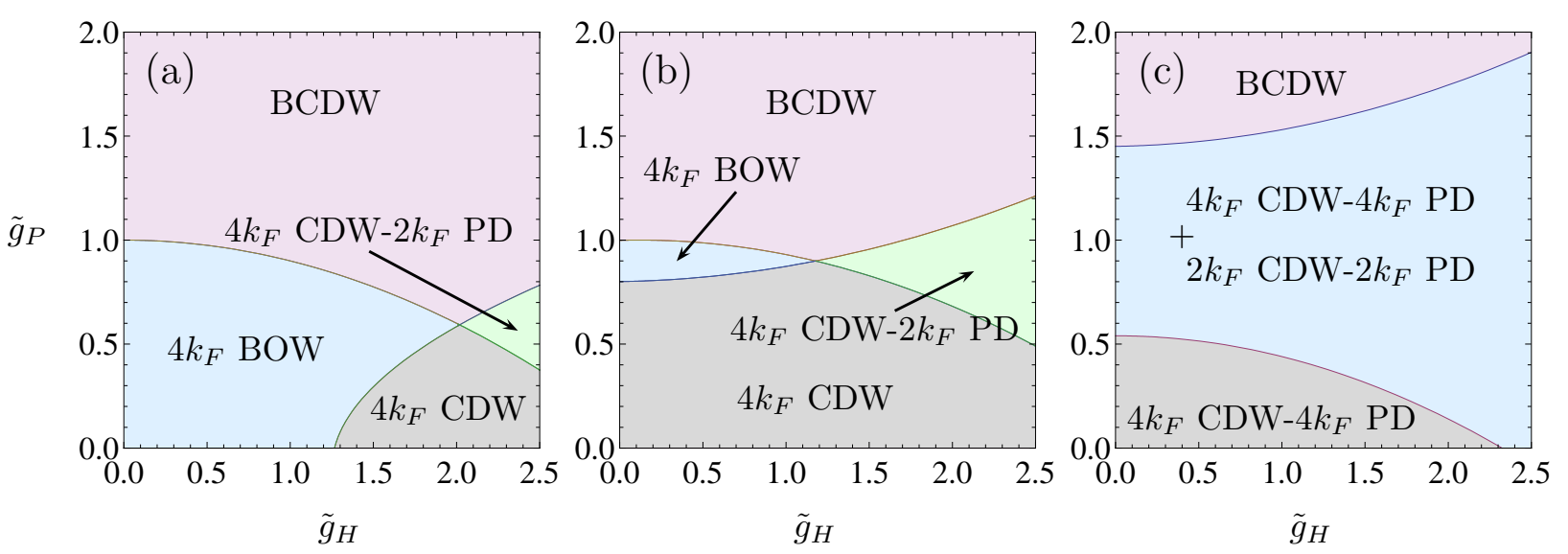

Figura 3.5: Diagrama de fases esquemático en el plano $\tilde{g}_{P}$ vs $\tilde{g}_{H}$ para diferentes valores de $\tilde{g}_{V}$. (a) $\tilde{g}_{1 / 4}=1, \tilde{g}_{V}=0$; (b) $\tilde{g}_{1 / 4}=-3, \tilde{g}_{V}=0$; (c) $\tilde{g}_{1 / 4}=-3, \tilde{g}_{V}=0.2$. Los bordes de las fases se obtienen a partir del cruce de las diferentes soluciones en la figura (3.4). La fase con $\tilde{g}_{1 / 4}>0$ y $\tilde{g}_{V}>0$ es similar al caso $(a)$ y por lo tanto no se muestra aquí. El efecto de la deformación de Peierls en la repulsión coulombiana a primeros vecinos es más importante para $V$ grande, que es equivalente a fijar $\tilde{g}_{1 / 4}<0$.

la modulación de las constantes de acoplamiento si la interacción coulombiana es de corto alcance (ver ecuación (3.1)). Hemos implementado el método de bosonización abeliana para estudiar las propiedades de baja energía del modelo y posteriormente hemos realizado un análisis semiclásico de la teoría efectiva para identificar las posibles fases electro-elásticas del estado fundamental. Como resultado, encontramos varios tipos de ordenes de carga combinados con deformaciones de la red dependiendo de los parámetros microscópicos del sistema $U$, $V$ y de la magnitud de los acoplamientos electrón-fonón $\tilde{g}_{P}, \tilde{g}_{H}$ y $\tilde{g}_{V}$. En cada una de estas fases, se favorece una deformación elástica no trivial que agrupa bloques de dos o cuatro sitios, mientras que el sector de carga adopta una de las siguientes fases a temperatura cero:

- Para $U>4 V\left(\tilde{g}_{1 / 4}>0\right), \tilde{g}_{V}=0$ y para valores pequeños de $\tilde{g}_{P}$ y $\tilde{g}_{H}$, el estado fundamental tiene los enlaces dimerizados $4 k_{F}$ Bond-Order-wave (BOW) con una densidad de carga uniforme (figura 3.2(a)). Si $\tilde{g}_{P}$ es suficientemente grande ( $\tilde{g}_{H}$ fijo), hay una segunda dimerización, dejando un estado tipo BCDW ( una superposición de ordenes $2 k_{F}$ y $4 k_{F}$ BOWs), acompañados por el orden de carga $\bullet-\bullet-$ o - o como en la figura 3.2(b). Por otro lado, para valores grandes de $\tilde{g}_{H}$ y pequeños de $\tilde{g}_{P}$, se establece un orden $4 k_{F} \mathrm{CDW}$, con enlaces uniformes como en la figura 3.2(c). Si se incrementa el valor de $\tilde{g}_{P}$, el estado fundamental viene a $4 k_{F} \mathrm{CDW}-2 k_{F} \mathrm{SP}$, donde el orden de carga $\bullet-\bullet-$ o - o es acompañado por una distorsión de la red $2 k_{F}$ como en la figura $3.2(\mathrm{~d})$.

- Para $V>U / 4\left(\tilde{g}_{1 / 4}<0\right)$, la fase $4 k_{F}$ BOW disminuye debido a la fuerte repulsión coulombiana $V$ y las fases con orden de carga dominan el diagrama de fases.

- Para $V>U / 4\left(\tilde{g}_{1 / 4}<0\right), \tilde{g}_{V}>0$, la fase $4 k_{F}$ BOW desaparece porque la repulsión de coulombiana entre primeros vecinos se incrementa como consecuencia de la deformación 
de Peierls. Las fases CDW poseen una pequeña contribución del orden $4 k_{F}$ BOW combinada con el original $2 k_{F}$, produciendo dos nuevas fases: $4 k_{F} \mathrm{CDW}-4 k_{F}$ SP y $4 k_{F}$ $\mathrm{CDW}-4 k_{F} \mathrm{SP}+2 k_{F} \mathrm{CDW}-2 k_{F} \mathrm{SP}$ 


\section{Capítulo 4}

\section{Modelo de Heisenberg acoplado con la red}

\subsection{Introducción}

El estudio de las excitaciones de baja energía de los sistemas cuánticos frustrados ha sido un campo muy investigado en las últimas décadas debido a que la estructura de las excitaciones de baja energía de estos sistemas se ve reflejada directamente en la cantidades observadas experimentalmente tales como la curva de magnetización.

Sin embargo, en general, no es fácil revelar el rol de la frustración en los sistemas cuánticos de espines teóricamente, donde las fluctuaciones cuánticas y la frustración afectan cooperativamente las excitaciones de baja energía.

De todos los sistemas frustrados, uno de los modelos más estudiados y paradigmáticos es la cadena de espines $J_{1}-J_{2}$ representada en la figura 4.1 conocida como cadena zigzag [94, 95, 96, 97, 98, 99, 100], y puede ser realizada en compuestos como $\mathrm{SrCuO}_{2}$ [101], $\mathrm{Cu}$ (ampy) $\mathrm{Br}_{2}$ [102], $\mathrm{F}_{2} \mathrm{PIMNH}[103]$ and $\left(\mathrm{N}_{2} \mathrm{H}_{5}\right) \mathrm{CuCl}_{3}$ [104].

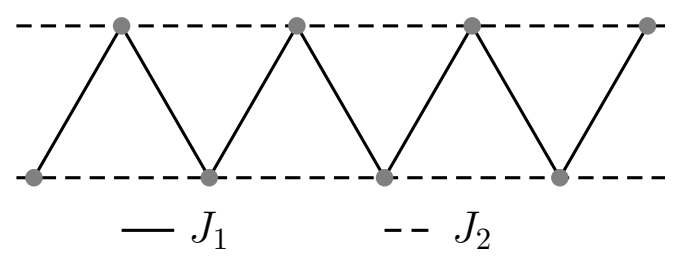

Figura 4.1: Cadena zig-zaz $J_{1}-J_{2}$ antiferromagnética. Los puntos en gris representan los sitios.

El hamiltoniano de la cadena zig-zag posee una estructura muy simple, sin embargo éste captura una gran variedad de efectos inducidos por la frustración. En efecto, la cadena zig-zag en un campo magnético ha sido estudiada activamente [105, 106, 107, 108, 109, 110, 111] mostrando un plateau o meseta de magnetización a $M=1 / 3$ acopañada de la rupura espontanea de la simetría de traslación. Estos estudios muestran que el estado fundamental describe una 
fase tipo Tomonaga-Luttinger (TL) para $0<J_{2} / J_{1}<\alpha_{c}=0.56^{1}$. Para $\alpha_{c}<J_{2} / J_{1} \lesssim 1.25$ existe un plateau en la curva de magnetización en $M=1 / 3$. En la región del plateau, los estudios numéricos muestran que el estado fundamental es triplemente degenerado, donde la simetría de traslación es espontáneamente rota a una configuración de la forma $\{\uparrow, \uparrow, \downarrow\}$ (las otras posibles son $\{\uparrow, \downarrow, \uparrow\},\{\downarrow, \uparrow, \uparrow\}$ ). En la figura (4.2) se muestra el diagrama de fases del estado fundamental (izquierda) en función de la relación de acoplamientos y el campo magnético externo; en la figura (4.2) (derecha) se muestra como ejemplo la curva de magnetización para $J_{2} / J_{1}=0.7$ para una cadena de $N=192$ sitios mostrando el plateau de magnetización a $1 / 3$ del valor de saturación.
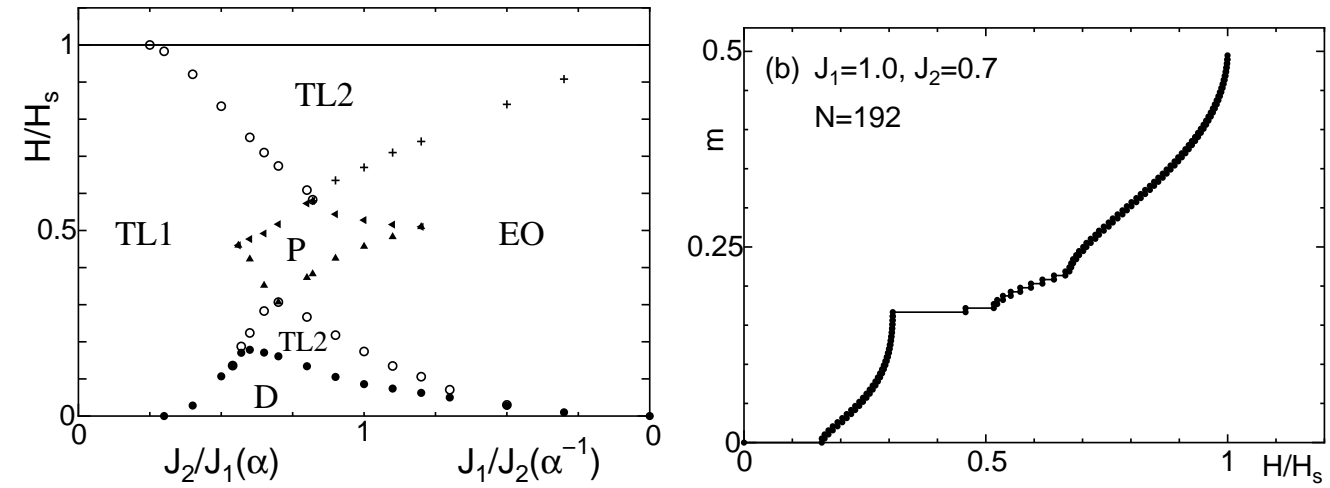

Figura 4.2: (izquierda) Diagrama de fases magnetico para la cadena $J_{1}-J_{2}$; (derecha) curva de magnetización para $J_{2} / J_{1}=0.7$ y $N=192$ sitios calculada por DMRG. Las fases TL1 y TL2, representan líquidos de Luttinger con diferente estructura mientras D indica una fase dimerizada. Figuras extraídas de K. Okunishi, T. Tonegawa, J. Phys. Soc. Jpn. 72 (2003) 479 .

Además de los compuestos antes menciondaos el compuesto $\mathrm{CuGeO}_{3}$ ha recivido una gran atención donde, además de la frustración, el acoplamiento de los espines con los fonones desempeña un rol crucial en la propiedades magnéticas [38]. Debido al interacciones que presenta el material antes mencionado, la competencia entre la modulación que puede presentar el acoplamiento magnético y la frustración ha estimulado considerables esfuerzos para estudiar sistemas puramente magnéticos, en particular a magnetización cero $[113,114,115,116,117]$. Recientemente se demostró que la inclusión de fonones en escaleras zig-zag frustradas puede abrir un plateau en la curva de magnetización, no solo a magnetización cero, sino también para otros valores racionales $M$ respecto de saturación $(M=1 / 3,1 / 2 \ldots)$ [118]. En tal situación, lo que se observa es una modulación en el acoplamiento de intercambio, con un patrón espacial asociado con la simetría de traslación del estado fundamental.

\footnotetext{
${ }^{1}$ El valor crítico para $J_{2} / J_{1}$ también ha sido reportado como 0.487 usando la técnica conocida como level spectroscopy, ver Ref. [112]
} 
En este capítulo investigamos las fases magnéticas de sistemas unidimensionales de espines $S=1 / 2$ en el límite de baja energía por medio de bosonización abeliana y diagonalización exacta.

En la primera parte estudiamos un sistema de trímeros de espines acoplados antiferromagnéticamente a magnetización $M=1 / 3$ y analizamos la competencia entre modulación de las constantes de acoplamiento magnético y frustración geométrica. El análisis lo abordamos desde dos límites particulares: (i) el límite de frustración fuerte que corresponde a la situación de trímeros débilmente acoplados y donde la frustración tiene consecuencias drásticas en las propiedades del estado fundamental; y (ii) el límite de modulación débil que corresponde a la situación de acoplamientos cuasi-homogéneos, y de esta manera el problema posee una relación directa con la cadena zig-zag. Como veremos la competencia entre modulación y frustración conduce a diversas fases magnéticas que se establecen en el estado fundamental. En la segunda parte, continuando con las ideas previamente desarrolladas estudiamos la cadena antiferromagnética $J_{1}-J_{2}$ acoplada con fonones en el límite adiabático a magnetización $M=1 / 3$, con el objetivo de identificar las posibles fases magnetoelásticas del estado fundamental en función del grado de frustración del sistema, e intentamos revelar si la inclusión de los fonones puede producir nuevas fases magnéticas.

\subsection{Transiciones de fase cuánticas en cadenas trimeri- zadas.}

Para ilustrar las consecuencias de la presencia de frustración magnética vamos a analizar el sistema de espines ( de magnitud $S$ ) frustrado más sencillo: un trímero de espines acoplados antiferromagnéticamente como se muestra en la figura 4.3.

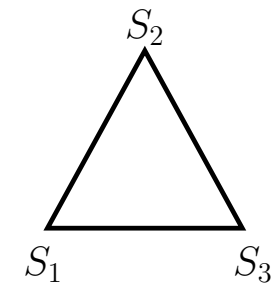

Figura 4.3: Trímero de espines acoplados antiferromagneticamente.

El hamiltoniano de Heisenberg del trímero está dado por

$$
\mathcal{H}_{0}=\sum_{i<j} J \mathbf{S}_{i} \mathbf{S}_{j}, \quad i=1,2,3 .
$$

donde $\mathbf{S}_{i}$ es el operador de espín en el sitio $i$-ésimo y $J$ es la constante de acoplamiento antiferromagnética $J>0$. Dado a que los acoplamientos entre los espines son iguales el hamiltoniano se puede reescribir en términos del operador de espín total $\mathbf{S}^{T}=\mathbf{S}_{1}+\mathbf{S}_{2}+\mathbf{S}_{3}$,

$$
\mathcal{H}_{0}=\frac{J}{2}\left(\mathbf{S}_{1}+\mathbf{S}_{2}+\mathbf{S}_{3}\right)^{2}-\frac{3}{2} J S(S+1)
$$


Las dimensiones de las representaciones en las que se descompone el producto directo de los tres espines, se pueden obtener vía la descomposición de Clebsh-Gordan. Así para espín $S=1 / 2$ tenemos

$$
\frac{1}{2} \otimes \frac{1}{2} \otimes \frac{1}{2}=\frac{1}{2} \oplus \frac{1}{2} \oplus \frac{3}{2}
$$

mostrando que el estado fundamental es cuatro veces degenerado, puesto que esta formado por dos subespacios de dimensión igual a $2 \frac{1}{2}+1=2$.

Claramente el conjunto de operadores que conmutan $\mathcal{H}_{0},\left(\mathbf{S}^{T}\right)^{2}, S_{z}^{T}$ no es completo puesto que solo permiten distinguir seis de los ocho autoestados del hamiltoniano. La degeneración extra está asociada a la simetría de permutación de los sitios del trímero. Esto se puede apreciar definiendo los operadores de permutación, $P_{1}, P_{2}$ y $P_{3}$ dados por

$$
P_{1}=2\left(\frac{1}{4}+\mathbf{S}_{2} \cdot \mathbf{S}_{3}\right), \quad P_{2}=2\left(\frac{1}{4}+\mathbf{S}_{1} \cdot \mathbf{S}_{3}\right), \quad P_{3}=2\left(\frac{1}{4}+\mathbf{S}_{1} \cdot \mathbf{S}_{2}\right)
$$

que satisfacen

$$
\left[S_{a}^{T}, P_{j}\right]=0 \quad j=1,2,3 \quad \mathrm{y} \quad a=x, y, z .
$$

Puesto que el operador de espín total conmuta con las seis permutaciones posibles de los tres espines, la doble degeneración de cada uno de los estados fundamentales (con un dado valor de $S_{z}^{T}= \pm \frac{1}{2}$ ) puede ser representada por operadores, asociados a una representación bidimensional del grupo de permutaciones. Asociado a tal simetría se introduce el operador pseudo-escalar $\tau$, definido como:

$$
\tau=\frac{2}{\sqrt{3}} \mathbf{S}_{1} \cdot\left(\mathbf{S}_{2} \times \mathbf{S}_{3}\right)
$$

que satisface:

$$
\left[\mathcal{H}_{0}, \tau\right]=0 .
$$

El observable asociado al operador $\tau$ se conoce como quiralidad u operador de pseudo-espín, ya que su signo distingue la orientación de la terna $\mathbf{S}_{1}, \mathbf{S}_{2}, \mathbf{S}_{3}$. De esta manera los autoestados del problema, se pueden identificar por el conjunto de números cuánticos asociados a los operadores:

$$
\left\{\mathcal{H}_{0},\left(\mathbf{S}^{T}\right)^{2}, S_{z}^{T}, \tau\right\}
$$

En la tabla (4.1) se detalla la relación entre los autoestados del problema y los estados de la base canónica

$$
B=\{|+++\rangle,|++-\rangle,|+-+\rangle,|+--\rangle,|-++\rangle,|-+-\rangle,|--+\rangle,|---\rangle\},
$$

junto con los autovalores de $\mathbf{S}^{T}$, de la componente $z$ del espín total y del operador quiralidad: 


$\begin{array}{ccrrr}\text { Estado } & & \text { Energía } & \mathbf{S}_{z}^{T} & \text { Quiralidad }\left(\tau_{z}\right) \\ |1\rangle & |+++\rangle & \frac{3}{4} J & \frac{3}{2} & 0 \\ |2\rangle & \frac{1}{\sqrt{3}}(|++-\rangle+|+-+\rangle+|-++\rangle) & \frac{3}{4} J & \frac{1}{2} & 0 \\ |3\rangle & \frac{1}{\sqrt{3}}(|++-\rangle+|+-+\rangle+|-++\rangle) & \frac{3}{4} J & -\frac{1}{2} & 0 \\ |4\rangle & |---\rangle & \frac{3}{4} J & -\frac{3}{2} & 0 \\ |5\rangle & \frac{1}{\sqrt{3}}\left(|+-+\rangle+w|++-\rangle+w^{2}|-++\rangle\right) & -\frac{3}{4} J & \frac{1}{2} & \frac{1}{2} \\ |7\rangle & \frac{1}{\sqrt{3}}\left(|+-+\rangle+w^{2}|++-\rangle+w|-++\rangle\right) & -\frac{3}{4} J & \frac{1}{2} & -\frac{1}{2} \\ |6\rangle & \frac{1}{\sqrt{3}}\left(|-+-\rangle+w|--+\rangle+w^{2}|+--\rangle\right) & -\frac{3}{4} J & -\frac{1}{2} & \frac{1}{2} \\ |8\rangle & \frac{1}{\sqrt{3}}\left(|-+-\rangle+w^{2}|--+\rangle+w|+--\rangle\right) & -\frac{3}{4} J & -\frac{1}{2} & -\frac{1}{2}\end{array}$

Cuadro 4.1: Autovectores del Hamiltoniano $\mathcal{H}_{0}$, caracterizados por los autovalores de la componente $z$ de los operadores de espín total y quiralidad de un triángulo $\left(w=e^{i \frac{2 \pi}{3}}\right)$.

Claramente cualquier combinación lineal de los cuatro estados $|5\rangle,|6\rangle,|7\rangle,|8\rangle$ se encuentra dentro del subespacio que forma el estado fundamental del problema. Si por ejemplo armamos la combinación lineal

$$
\frac{1}{\sqrt{2}}\left[i w^{2}|5\rangle-i w|7\rangle\right]
$$

el resultado que se obtiene es exactamente un singlete formado a partir de los sitios 1 y 2 mientras que el espín restante queda frustrado al no poder acomodarse con sus vecinos.

$$
\frac{1}{\sqrt{2}}\left(|+,-\rangle_{1,2}-|-,+\rangle_{1,2}\right)|+\rangle_{3}
$$

Así el efecto de la frustración de los espines del triángulo es el de acomodar un singlete entre dos de ellos cualesquiera y dejar libre el tercero para minimizar la energía. De manera análoga se pueden armar otras combinaciones lineales para formar singletes entre dos sitios dejando la libertad de estar $\uparrow$ o $\downarrow$ al espín restante.

Notemos además que el subespacio correspondiente al estado fundamental posee una proyección $z$ del espín total igual a $\pm 1 / 2$, mientras que la máxima proyección posible en un triángulo es $\pm 3 / 2$. Esto quiere decir que el valor absoluto de la magnetización normalizada respecto de saturación (del valor máximo) corresponde a $M=1 / 3$,

$$
\begin{aligned}
\text { magnetización normalizada } & =\frac{\left| \pm \frac{1}{2}\right|}{\left| \pm \frac{3}{2}\right|} \\
& =\frac{1}{3} .
\end{aligned}
$$

Motivados por las ideas y los resultados presentados en las secciones anteriores surgen algunas preguntas:

- ¿ Cómo modifica la estructura magnética del estado fundamental la inclusión de la modulación de los acoplamientos magnéticos en un sistema de $N$ espines acoplados?, y i cómo influye la frustración en las características del estado fundamental? 
- ¿. Es posible que las distintas fases magnéticas que surgen debido a la modulación de los acoplamientos se establezcan debido a la interacción con los espines con fonones en el límite adiabático? y de ser el caso, ¿ qué tipo de orden magneto-elástico es seleccionado?.

\subsubsection{Frustración vs modulación}

En esta sección nos concentraremos en de la competencia entre la modulación del acoplamiento magnético y la frustración en una escalera de espines tipo zig-zag con modificada debido a la presencia de modulaciones de las constantes de acoplamientos que rompen la simetría de traslación en el sector de magnetización $M=1 / 3$.

Como mencionamos en la introducción del capítulo en ausencia de modulación (es decir la cadena zig-zag con acoplamientos $J_{1}-J_{2}$ como en la figura (4.1)), el modelo presenta un plateau de magnetización a $M=1 / 3$. Además, el estado fundamental es tres veces degenerado y la simetría de traslación en un sitio de red está espontáneamente rota hacia una configuración del tipo $\uparrow \uparrow \downarrow$. Este resultado fue obtenido numéricamente [111] mostrando que los valores de espectación en el estado fundamental de los operadores $S_{i}^{z}$ presentan una configuración similar a la que se muestra en la figura (4.4). Basándonos en estos resultados, una

$$
\begin{aligned}
& \left|\mathrm{Née}_{1}\right\rangle=\cdots \uparrow \downarrow \uparrow \uparrow \downarrow \uparrow \uparrow \downarrow \uparrow \uparrow \downarrow \uparrow \cdots \\
& \left|\mathrm{Née}_{2}\right\rangle=\cdots \uparrow \downarrow \downarrow \uparrow \uparrow \downarrow \uparrow \uparrow \downarrow \uparrow \uparrow \downarrow \cdots \\
& \left|\mathrm{Née}_{3}\right\rangle=\cdots \downarrow \uparrow \uparrow \downarrow \uparrow \uparrow \downarrow \uparrow \uparrow \downarrow \uparrow \uparrow \cdots
\end{aligned}
$$

Figura 4.4: $\mid$ Néll $\rangle_{1,2,3}$ representan las configuraciones de los valores de espectación de $S_{i}^{z}$ en el estado fundamental triplemente degenerado.

deformación de las posiciones de los sitios de la red viene dada por un patrón de período tres de la forma

$$
u_{i}=\delta \sin \left(\frac{2 \pi}{3} i-\Phi_{0}\right)
$$

donde $u_{i}$ representa la coordenada escalar relevante que describe el desplazamiento del iésimo sitio secuencialmente numerado en una cadena unidimensional. En particular, nos vamos a concentrar en una deformación con amplitud $\delta>0$ y una fase $\Phi_{0}=-\frac{\pi}{3}$ causa que los sitios 1 y 3 se acerquen al sitio 2, y así sucesivamente con los demás sitios como se muestra en la figura (4.5). También vamos a asumir que los acoplamientos magnéticos varían linealmente con los desplazamientos relativos para poder estimar la modulación resultante tanto para los acoplamientos a primeros vecinos como a segundos vecinos. De esta manera, los sitios 1, 2 y 3 están magnéticamente acoplados en igual amplitud $J$ formando trímeros. Las líneas discontinuas son más débiles y la identificamos por $J^{\prime}$.

Notemos que esta modulación de los acoplamientos selecciona trímeros con acoplamientos internos fuertes como unidades básicas, los cuales están débilmente acoplados entre ellos. La situación limite, en el cual los trímeros se encuentran débilmente acoplados será considerada 


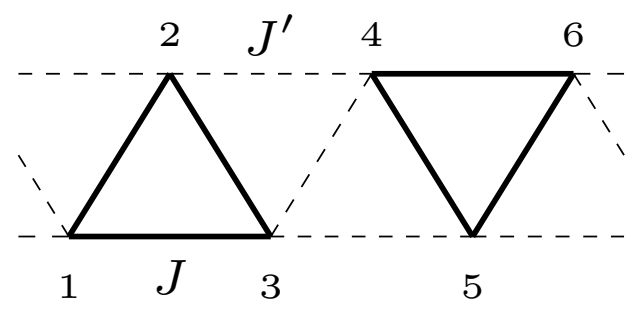

Figura 4.5: Descripción esquemática de la escalera de espines trimerizada. En este caso, tanto los acoplamientos a primeros como a segundos vecinos son modulados.

en la siguiente sección y veremos como la frustración genera un plateau de magnetización. Por otro lado, en la situación con $\delta<0$ (en este caso los acoplamientos favorecidos pasan a ser las líneas discontinuas en la figura (4.5) ) el sistema corresponde a una cadena de espines con acoplamientos mayores a primeros vecinos y menores a segundos, terceros y cuartos. El limite $J / J^{\prime}=0$ es la usual cadena de espines, la cual no presenta ningún plateau en la curva de magnetización.

Basados en este análisis, vamos a investigar la estructura magnética del estado fundamental a magnetización $M=1 / 3$ del sistema antes mencionado en el caso de espín $S=1 / 2$. El hamiltoniano de Heisenberg con una modulación de los acoplamientos de periodo tres tanto a primeros como a segundos vecinos está dado por

$$
H=\sum_{i}\left(J_{i, i+1} \mathbf{S}_{i} \cdot \mathbf{S}_{i+1}+J_{i, i+2} \mathbf{S}_{i} \cdot \mathbf{S}_{i+2}\right)
$$

donde $\mathbf{S}_{i}$ denota el operador de espín en el sitio $i$-ésimo. La modulación está dada por acoplamientos a primeros vecinos $J_{i, i+1}>0$ que siguen la secuencia $\left\{J, J, J^{\prime}, J, J, J^{\prime}, \ldots\right\}$ formando una secuencia de periodo tres como la que se muestra en la figura (4.5), y acoplamientos $J_{i, i+2}>0$ también formando una secuencia de periodo tres de la forma $\left\{J, J^{\prime}, J^{\prime}, J, J^{\prime}, J^{\prime}, \ldots\right\}$ como la que se muestra en la figura (4.5).

Como veremos la competencia entre frustración y modulación da como resultado un rico diagrama de fases para el estado fundamental incluyendo un número de transiciones de fases de distintos órdenes, incluyendo estados como el plateau clásico donde los valores de espectación de $\left\langle S_{i}^{z}\right\rangle$ poseen una estructura similar a up-up-down; plateau cuántico y estados quirales. Resumiendo, veremos que el sistema adopta un único estado fundamental up-up-down para $\alpha=J^{\prime} / J>1$ separado por una transición de fase de primer orden en $\alpha_{3}=1$ hacia una fase $Z_{2}$ que se extiende hasta $\alpha$ ligeramente menor que 1 . En algún valor finito $\alpha_{2}$ encontramos una transición de fase de segundo orden tipo Ising a un único estado tipo plateau cuántico. En el límite de frustración fuerte $\alpha \ll 1$, encontramos otra transición en $0<\alpha_{1}<\alpha_{2}$ hacia una fase crítica quiral con magnetización uniforme en el estado fundamental. El diagrama de fases del estado fundamental final se muestra en la figura (4.6). El análisis se basa en bosonización abeliana, teoría de perturbaciones por bloques y diagonalización exacta de sistemas pequeños utilizando el algoritmo de Lanczos. 


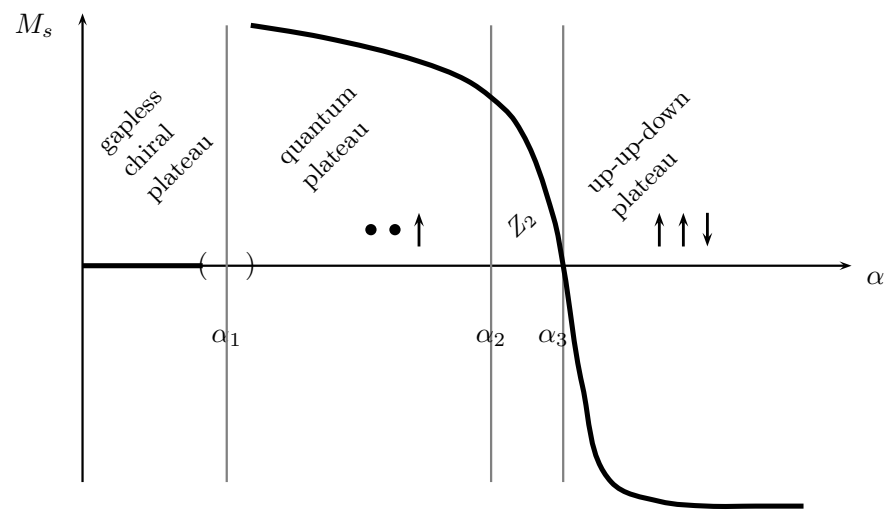

Figura 4.6: Diagrama de fases esquemático del estado fundamental del sistema de espines mostrado en la figura 4.5. $M_{s}$ corresponde al parámetro de orden magnético dado por la ecuación 4.32 .

\subsubsection{Límite de frustración fuerte}

Para poder analizar el estado fundamental del sistema en el limite de frustración fuerte vamos a considerar el sistema como consistiendo de trímeros frustrados de espín $S=1 / 2$ débilmente acoplados antiferromagnéticamente por $J^{\prime}>0$. Por conveniencia, en esta sección

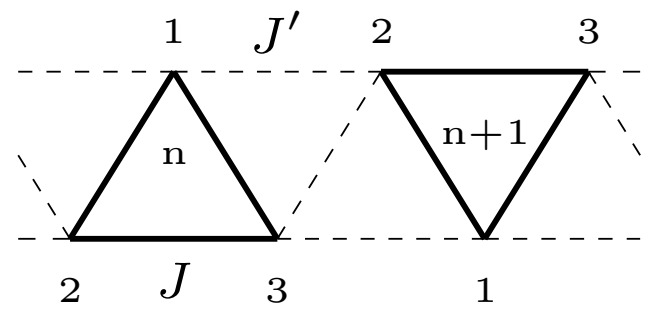

Figura 4.7: Descripción esquemática del sistema en el régimen de frustración fuerte, con $J^{\prime} \ll J$ indicado por trímeros de líneas gruesas.

vamos a enumerar los trímeros con un índice $n$ y vamos renombrar los espines como $\mathbf{S}_{n}^{a}$ ( $a=1,2,3$ dentro de cada trímero, ver figura 4.7) para escribir el hamiltoniano (4.13) como

$$
\begin{aligned}
H & =\sum_{n} J\left(\mathbf{S}_{n}^{1} \cdot \mathbf{S}_{n}^{2}+\mathbf{S}_{n}^{2} \cdot \mathbf{S}_{n}^{3}+\mathbf{S}_{n}^{3} \cdot \mathbf{S}_{n}^{1}\right) \\
& +\sum_{n} J^{\prime}\left(\mathbf{S}_{n}^{1} \cdot \mathbf{S}_{n+1}^{2}+\mathbf{S}_{n}^{3} \cdot \mathbf{S}_{n+1}^{1}+\mathbf{S}_{n}^{3} \cdot \mathbf{S}_{n+1}^{2}\right) .
\end{aligned}
$$

Experimentalmente existen candidatos de sistemas similares en cuanto a la estructura de acoplamientos conocidos como tubos de espines [119]. Entre ellos podemos mencionar el óxido de vanadio $\mathrm{Na}_{2} \mathrm{~V}_{3} \mathrm{O}_{7}[120,121,122]$ que corresponde a un tubo de espines de nueve 
patas (figura 4.8a), y el compuesto $\left[\left(\mathrm{CuCl}_{2} \operatorname{tachH}\right)_{3} \mathrm{Cl}\right] \mathrm{Cl}_{2}[123,124]$ formado por tres filas de espines acoplafos como se muestra en la figura 4.8a.

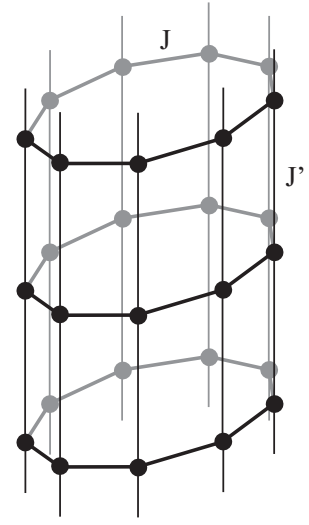

(a)

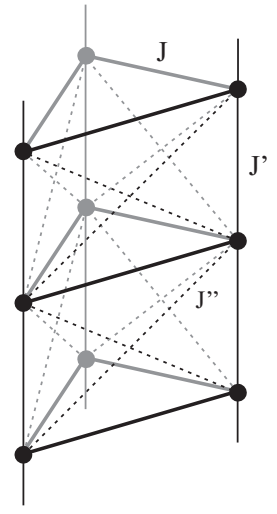

(b)

Figura 4.8: Modelos de tubos de espín (a) $\mathrm{Na}_{2} \mathrm{~V}_{3} \mathrm{O}_{7}$ y (b) $\left[\left(\mathrm{CuCl}_{2} \text { tachH }\right)_{3} \mathrm{Cl}\right] \mathrm{Cl}_{2}$.

Volviendo a nuestro caso, para estudiar el límite $J^{\prime} \ll J$ del hamiltoniano (4.14) vamos a realizar un desarrollo perturbativo por bloques $[125,126]$ alrededor del estado fundamental exponencialmente degenerado de trímeros desacoplados $\left(J^{\prime}=0\right)$. A bajas energías y en el subespacio de espín total de cada trímero $S^{T}=1 / 2$ (correspondiente al subespacio del estado fundamental), los operadores de espín en cada vértice de un trímero pueden ser factorizados en términos del espín total y del operador de pseudo-espin (ecuación (4.5) ) como

$$
\mathbf{S}_{n}^{a}=\frac{2}{3} \mathbf{S}_{n}^{T}\left(\frac{1}{2}-T_{n}^{a}\right),
$$

donde

$$
\mathbf{S}_{n}^{T}=\mathbf{S}_{n}^{1}+\mathbf{S}_{n}^{2}+\mathbf{S}_{n}^{3}
$$

es el operador de espín total del $n$-ésimo trímero proyectado en el sector de baja energía. Los operadores $T_{n}^{a}$ actúan sobre el sector quiral como

$$
\begin{aligned}
& T_{n}^{1}=\tau_{n}^{+}+\tau_{n}^{-}, \\
& T_{n}^{2}=\omega^{2} \tau_{n}^{+}+\omega \tau_{n}^{-}, \\
& T_{n}^{3}=\omega \tau_{n}^{+}+\omega^{2} \tau_{n}^{-},
\end{aligned}
$$

donde $\tau_{n}^{ \pm}=\tau_{n}^{x} \pm i \tau_{n}^{y}$ y $\omega=e^{i 2 \pi / 3}$.

Para estudiar el estado fundamental del sector de magnetización $M=1 / 3$, consideremos al sistema bajo la acción de campo magnético externo $h^{*}>0$ que selecciona el subespacio de $\left\langle S_{n}^{z, T}\right\rangle=1 / 2$ en cada trímero, pero que es lo suficientemente pequeño como para descartar excitaciones de espín $S=3 / 2$ en cada trímero (figura 4.9). Bajo esta restricción, la magnetización total del sistema es $M=1 / 3$ dejando como hamiltoniano efectivo el de una cadena de pseudo-espines describiendo excitaciones no-magnéticas. 


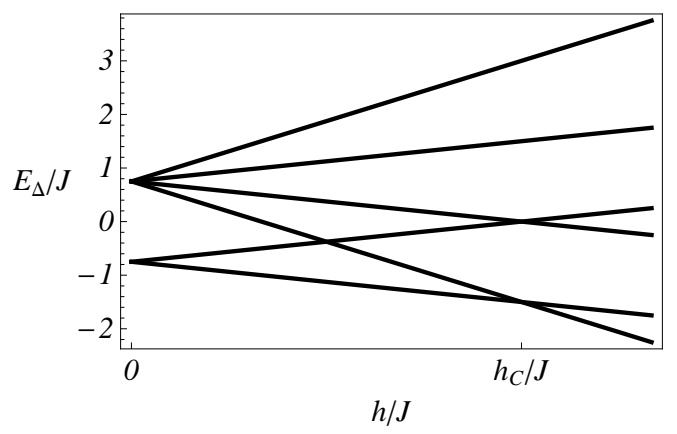

Figura 4.9: Evolución de los ocho niveles de energía del tr'mero de espines en función del campo magnético externo $h$.

A primer orden en perturbaciones por bloques [125], se obtiene un hamiltoniano del tipo $X Y$ con interacciones a primeros vecinos,

$$
H_{\text {eff }}^{(1)}=\frac{\sqrt{3}}{9} J^{\prime} \sum_{n}\left(e^{-i \frac{\pi}{6}} \tau_{n}^{+} \tau_{n+1}^{-}+e^{i \frac{\pi}{6}} \tau_{n+1}^{+} \tau_{n}^{-}\right)+c t e .
$$

Realizando una transformación de gauge

$$
\begin{aligned}
\tau_{n}^{+} & \rightarrow e^{-i n \frac{\pi}{6}} \tau_{n}^{+}, \\
\tau_{n}^{z} & \rightarrow \tau_{n}^{z},
\end{aligned}
$$

que preserva las relaciones de conmutación de $S U(2)$, se logra eliminar las fases en el hamiltoniano (4.18). Luego, utilizando las transformación de Jordan-Wigner se puede mapear las excitaciones no magnéticas quirales en un teoría de fermiones libres sin espín. El estado fundamental es entonces descrito por una banda semi-llena de fermiones.

Este modelo efectivo, describe las excitaciones no magnéticas del estado fundamental, que forman un espectro continuo sin gap, y estado fundamental no degenerado. Usando las ecuaciones (4.16), (4.17) y(4.19) uno puede evaluar valores de expectación de diferentes funciones de correlación. La densidad de espín en la dirección paralela al campo aplicado es uniforme y simplemente igual al promedio de magnetización,

$$
\left\langle S_{n}^{z a}\right\rangle=1 / 6
$$

mientras que la componente en el plano transversal se anula. La función de correlación espín-espín en la dirección paralela al campo externo está dada por:

$$
\left\langle S_{n}^{z a} S_{m}^{z b}\right\rangle=\frac{1}{36}+\frac{\text { const }}{\sqrt{n-m}} \cos \left((n-m) \frac{\pi}{6}+\frac{2 \pi}{3}(a-b)\right)
$$

con $a, b=1,2,3$, mostrando orden de largo alcance (cada trímero en un estado de espín $+1 / 2$ ), más oscilaciones con decaimiento algebraico. En el plano $X Y$, la correlación espínespín decaen exponencialmente. Estos resultados son de esperar debido a que las excitaciones 
no magnéticas no poseen gap en el espectro.

De la discusión anterior, uno puede establecer que para $J^{\prime} \ll J$ el sistema de espines a $M=1 / 3$ presenta una fase sin gap correspondiente a los grados de libertad quirales no magnéticos describiendo un líquido de Luttinger con $K=1$.

Para poder estudiar una región más extensa alrededor de $J^{\prime} / J=0$ calculamos el siguiente orden perturbativo . La derivación requiere un cálculo más elaborado y tedioso, y provee interacciones a segundos vecinos. Saltando los detalles, la corrección a segundo orden toma la forma:.

$$
\begin{aligned}
H_{e f f}^{(2)} / J= & \alpha^{2}\left\{\frac{2}{27} \sum_{n}\left[\tau_{n}^{+}+\tau_{n}^{-}\right]+\right. \\
& +\frac{5}{162} \sum_{n}\left[e^{-i \frac{2 \pi}{3}} \tau_{n}^{+} \tau_{n+1}^{-}+e^{i \frac{2 \pi}{3}} \tau_{n+1}^{+} \tau_{n}^{-}\right]- \\
& -\frac{1}{3} \sum_{n} \tau_{n}^{z} \tau_{n+1}^{z}+ \\
& +\frac{2}{81} \sum_{n}\left[e^{i \frac{2 \pi}{3}} \tau_{n}^{+} \tau_{n+2}^{-}+e^{-i \frac{2 \pi}{3}} \tau_{n+2}^{+} \tau_{n}^{-}\right]- \\
& -\frac{2}{27} \sum_{n}\left[\tau_{n}^{+} \tau_{n+1}^{+}+\tau_{n+1}^{-} \tau_{n}^{-}\right]- \\
& \left.-\frac{4}{81} \sum_{n}\left[\tau_{n}^{+} \tau_{n+1}^{+} \tau_{n+2}^{+}+\tau_{n+2}^{-} \tau_{n+1}^{-} \tau_{n}^{-}\right]\right\}+ \text {cte }
\end{aligned}
$$

donde $\alpha=J^{\prime} / J$. Los términos en la segunda línea son similares a aquellos que surgen del primer orden en la ecuación (4.18), para lo cual proponemos una transformación de gauge diferente para eliminar las fases en los términos $X Y$

$$
\begin{aligned}
& \tau_{n}^{+} \rightarrow e^{i \lambda(\alpha) n} \tau_{n}^{+}, \\
& \tau_{n}^{z} \rightarrow \tau_{n}^{z},
\end{aligned}
$$


donde $\lambda(\alpha)=\arctan \left[\frac{\sqrt{3}(5 \alpha-18)}{5 \alpha+54}\right]$, quedando el hamiltoniano efectivo de la siguiente forma

$$
\begin{aligned}
H_{e f f} / J= & -\rho(\alpha) \sum_{n}\left[\frac{1}{2}\left(\tau_{n}^{+} \tau_{n+1}^{-}+\tau_{n+1}^{+} \tau_{n}^{-}\right)+\Delta^{z}(\alpha) \tau_{n}^{z} \tau_{n+1}^{z}\right]+ \\
& +\frac{2}{81} \alpha^{2} \sum_{n}\left[e^{-i 2\left(\lambda_{(\alpha)}-\frac{\pi}{3}\right)} \tau_{n}^{+} \tau_{n+2}^{-}+h . c .\right]+ \\
& +\frac{2}{27} \alpha^{2} \sum_{n}\left[e^{i \lambda_{(\alpha)} n} \tau_{n}^{+}+h . c .\right]- \\
& -\frac{2}{27} \alpha^{2} \sum_{n}\left[e^{i \lambda_{(\alpha)}(2 n+1)} \tau_{n}^{+} \tau_{n+1}^{+}+\text {h.c. }\right]- \\
& -\frac{4}{81} \alpha^{2} \sum_{n}\left[e^{i 3 \lambda_{(\alpha)}(n+1)} \tau_{n}^{+} \tau_{n+1}^{+} \tau_{n+2}^{+}+\text {h.c. }\right]
\end{aligned}
$$

donde $\Delta^{z}(\alpha)=\frac{\alpha^{2}}{3 \rho(\alpha)}$ y $\rho(\alpha)=\alpha / 81 \sqrt{972+25 \alpha^{2}}$.

En la ecuación (4.25) la primera y segunda línea representan un modelo de Heisenberg anisotrópico con interacciones a primeros y segundos vecinos para los operadores de pseudoespín. Los acoplamientos a primeros vecinos poseen anisotropía dada por $\Delta^{z}(\alpha)$, mientras que los acoplamientos de segundos vecinos son únicamente $X Y$. De las expresiones de los coeficientes se observa que $0<\Delta^{z}(\alpha)<1$ y que los acoplamientos a segundos vecinos son mas pequeños que los de primeros vecinos. Todos los otros términos incluyen fases que dependen de la posición, y se cancelan en el límite termodinámico, restaurando la simetría $U(1)$. Esta cancelación de los términos oscilatorios, se puede ver más claramente utilizando bosonización para describir el comportamiento de baja energía.

Aplicando el procedimiento estándar de bosonización derivamos el hamiltoniano de baja energía,

$$
\begin{aligned}
H_{e f f} \approx & \frac{v}{2} \int d x\left[\frac{1}{K}\left(\partial_{x} \varphi\right)^{2}+K\left(\partial_{x} \theta\right)^{2}\right] \\
& -\Gamma \int d x \cos [2 \sqrt{4 \pi} \varphi]
\end{aligned}
$$

donde la velocidad de Fermi, el parámetro de Luttinger y el acoplamiento $\Gamma$ dependen de $J^{\prime} / J$. Para $J^{\prime} / J$ pequeño, la perturbación armónica es fuertemente irrelevante, y la teoría efectiva describe una fase sin gap como se obtuvo mediante la teoría de perturbaciones por bloque a primero orden. A medida que incrementamos $J^{\prime} / J$, la dimensión conforme de la perturbación armónica disminuye, y según la ecuación (4.26) uno espera un transición de segundo orden tipo Berezinskii-Kosterlitz-Thouless (BKT), hacia una fase masiva. Un cálculo detallado de la dependencia de la dimensión conforme con respecto a $J^{\prime} / J$ muestra que la perturbación se vuelve relevante para $J^{\prime} / J \sim 0.5$. En la siguiente sección vamos a estudiar el problema ahora desde el otro límite correspondiente a modulaciones débiles, es decir $J^{\prime} / J \sim 1$, en donde ya no es posible aplicar el método perturbativo. 


\subsubsection{Límite de modulación débil}

Para estudiar la región $J^{\prime} \sim J$ es conveniente enumerar de una manera distinta los sitios para facilitar la notación. Para ello vamos a utilizar la descripción dada en la figura (4.5). En ese caso, el hamiltoniano microscópico para el sistema de espines se puede reescribir como

$$
\begin{aligned}
H= & \sum_{i}\left[\left(J-\frac{\epsilon}{2}\right) \mathbf{S}_{i} \cdot \mathbf{S}_{i+1}+(J-\epsilon) \mathbf{S}_{i} \cdot \mathbf{S}_{i+2}\right] \\
& -\epsilon \sum_{i} \cos \left(i \frac{2 \pi}{3}\right) \mathbf{S}_{i} \cdot \mathbf{S}_{i+1} \\
& +\epsilon \sum_{i} \cos \left((i-1) \frac{2 \pi}{3}\right) \mathbf{S}_{i} \cdot \mathbf{S}_{i+2}
\end{aligned}
$$

donde $\epsilon=2\left(J-J^{\prime}\right) / 3$.

La primera línea describe una cadena homogénea con $J_{1}=J-\epsilon / 2$ y $J_{2}=J-\epsilon$, mientras que el resto es una perturbación modulada de periodo tres. En el sector de magnetización $M=1 / 3$, implementando el método de bosonización abeliana descrito en la sección 2.1.4, la parte homogénea "es bien descrita" [46, 93] en términos de un hamiltoniano seno-Gordon dado por:

$$
H_{0}=\frac{v}{2} \int d x\left[\frac{1}{K}\left(\partial_{x} \varphi\right)^{2}+K\left(\partial_{x} \theta\right)^{2}\right]-g \cos [3 \sqrt{4 \pi} \varphi]
$$

donde $\varphi$ es un campo bosónico real, $v$ es la velocidad de ondas de espín, $\theta$ es el campo dual definido por $\partial_{x} \theta=\partial_{t} \varphi$ y $K$ es el arámetro de Luttinger. La presencia del tercer armónico en la Eq. (4.28) surge del triple proceso de dispersión Umklapp, con momento $k_{F}=\pi / 3$ fijo a magnetización $M=1 / 3$. Es conocido numéricamente [111, 112] que el hamiltoniano magnético homogéneo $H_{0}$ describe una fase tipo Tomonaga-Luttinger (TL) para $0<J_{2} / J_{1}<\alpha_{c}=0.56$. Para $\alpha_{c}<J_{2} / J_{1} \lesssim 1.25$ existe un plateau en la curva de magnetización en $M=1 / 3$. En término de los parámetros de la bosonización, para que ocurra esto debe ser $K_{L}>2 / 9$ para $J_{2} / J_{1} \lesssim \alpha_{c}$, ya que esto implica que la perturbación en Eq. (4.40) sea irrelevante. Entonces el coeficiente $g_{3}$ "fluye" a cero al aplicar el grupo de renormalización y la teoría efectiva describe una fase TL. Por otro lado, para $\alpha_{c} \lesssim J_{2} / J_{1} \lesssim 1.25$, debe ser $K_{L}<2 / 9$, haciendo (4.40) una perturbación relevante. Entonces éste término abre un gap en el espectro de excitaciones magnéticas generando un plateau en la curva de magnetización [46] observada en este rango. Más aún, en la región con gap, los estudios numéricos muestran que el estado fundamental es triplemente degenerado, donde la simetría de traslación es espontáneamente rota a una configuración de la forma $\{\uparrow, \uparrow, \downarrow\}$ [111](las otras posibles son $\{\uparrow, \downarrow, \uparrow\},\{\downarrow, \uparrow, \uparrow\}$ ). Tales configuraciones son descritas al fijar el campo bosónico en uno de los mínimos de la ecuación (4.40) considerado como la energía potencial semiclásica [46] siempre que $g_{3}>0$.

Consideremos ahora un análisis semiclásico de la teoría similar al presentado en el capítulo 3. Dentro de esta aproximación, el tercer armónico $-g \cos [3 \sqrt{4 \pi} \varphi]$ es considerado como un potencial; éste tiene posee tres mínimos no equivalentes para el bosón $\varphi$, como se muestra en 


\section{Transiciones de fase cuánticas en cadenas trimerizadas.}

la figura 4.10 indicando que el estado fundamental es triplemente degenerado en acuerdo con los resultados numéricos previos utilizando DMRG [111]. El mapeo de las variables bosónicas

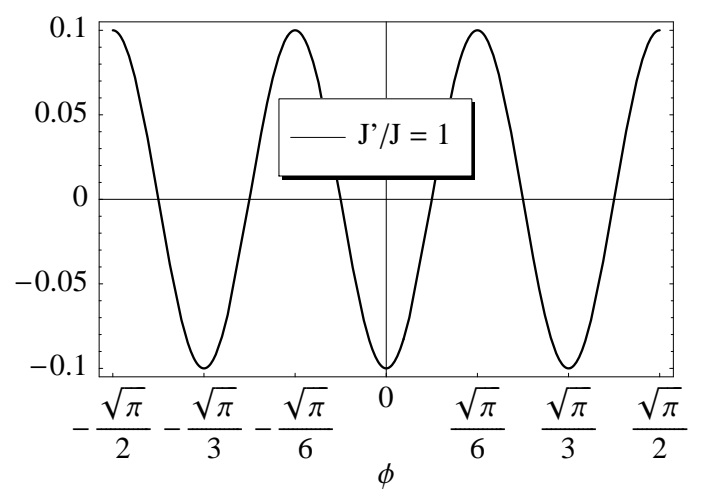

Figura 4.10: Potencial semiclásico para el sistema homogéneo $J=J^{\prime}$ (en unidades arbitrarias). Hay tres mínimos en el rango compactificado $\left(-\frac{\sqrt{\pi}}{2}, \frac{\sqrt{\pi}}{2}\right)$, correspondiendo las los tres estados no equivalentes mostrados en la figura (4.4).

a variables de espín [45], muestra que cada uno de los mínimos para $\varphi$ corresponde a los estados de plateau clásico mostrados en la figura (4.4) relacionados por traslaciones de la red $i \rightarrow i+1, i+2$. La segunda y tercer línea en la ecuación (4.27) representa una perturbación modulada al problema antes descrito. El punto crucial es que después utilizar las reglas de bosonización en estos términos se obtiene una contribución efectiva dada por

$$
H_{m o d} \sim \epsilon \int d x[C \cos [\sqrt{4 \pi} \varphi]-\cos [2 \sqrt{4 \pi} \varphi]]
$$

donde $C=1+\frac{2 \pi}{3}$. La dimensión conforme de estos armónicos es menor que la del tercer armónico, de esta manera resultan ser perturbaciones relevantes en la teoría. La teoría efectiva definida por $H_{0}+H_{m o d}$ es conocida como el modelo triple seno-Gordon [127]. Se han realizado numerosos análisis de la competencia entre diferentes armónicos principalmente del doble seno-Gordon [88, 90, 128, 129]. Para estudiar las propiedades del estado fundamental del modelo definido por las ecuaciones (4.28) y (4.29) vamos a realizar un análisis semiclásico considerando todas las perturbaciones relevantes.

Para $J^{\prime}>J$ el armónico básico domina el mínimo del potencial (figura 4.11, panel izquierdo), tal que la perturbación selecciona una única configuración $\varphi=0$ correspondiendo a uno de los estados $\{\uparrow, \uparrow, \downarrow\}$ (el que los espines $\downarrow$ están en los sitios $3 i+2$ figura 4.4). Por otro lado, un rico escenario ocurre para $J^{\prime}<J$ cuando el primer y segundo armónico entran en "conflicto" con tercer armónico (figura 4.11, panel derecho). En este caso el mínimo del potencial pasa, en una primera etapa, de ser triplemente a doblemente degenerado debido a una pequeña perturbación. De las tres posibles configuraciones para $\varphi$, se seleccionan dos corridas de sus configuraciones conmensuradas correspondientes a algún estado $\{\uparrow, \uparrow, \downarrow\}$. Esta fase está caracterizada por una simetría de reflexión $Z_{2}$, espontáneamente rota en cada uno de los mínimos del potencial. Cuando $J^{\prime} / J$ es inferior a un cierto valor finito, estos mínimos 

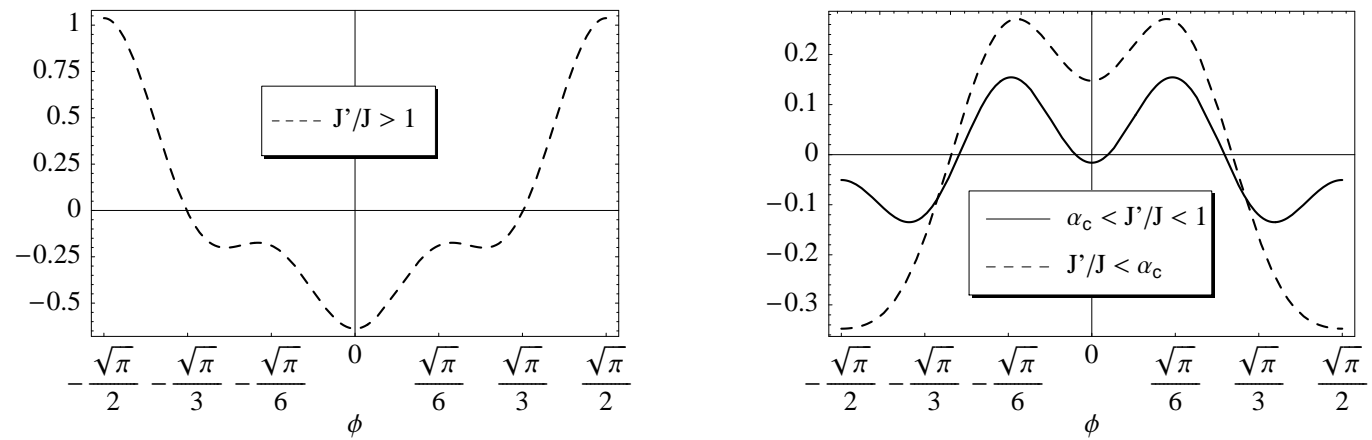

Figura 4.11: Modificación del potencial semiclásico debido a las perturbaciones relevantes. Para $J^{\prime}>J$ (panel izquierdo), el mínimo central fija el sistema en un estado particular up-updown. Para $J^{\prime}<J$ (panel derecho), la estructura del mínimo pasa primero a ser doblemente degenerado y entonces, a partir de un valor crítico de $J^{\prime} / J$, a un único estado fundamental desplazado en $\sqrt{\pi} / 6$ con respecto a la posición no perturbada. Este corrimiento selecciona un estado tipo plateau cuántico no conectado con los estadosup-up-down.

colapsan en un único mínimo en $\varphi=\sqrt{\pi} / 2 \equiv-\sqrt{\pi} / 2$, levantando la degeneración completamente. Más aún, la posición del mínimo es corrida hacia una configuración del campo que corresponde a un "máximo" en el caso homogéneo $\left(J^{\prime}=J\right)$. En términos de espines, el sistema adquiere una configuración muy diferente, conocida como estado de plateau cuántico cuya estructura se muestra en la figura 4.12. Esta configuración esta caracterizada por

$$
|\mathrm{PC}\rangle=\cdots \bullet \bullet \uparrow \bullet \uparrow \bullet \uparrow \cdots
$$

Figura 4.12: Configuración de plateau cuántico.

singletes alternando con sitios con espín $\uparrow$ (en nuestro caso, los espines $\uparrow$ se encuentran en los sitios $3 i+2$ en la figura 4.5). Dada la simetría y degeneración a ambos lados del punto critico, uno puede conjeturar que la transición pertenece a la clase de universalidad de Ising [129].

El presente análisis de la teoría bosonizada indica la presencia de una transición de fase de primer orden en el punto $J^{\prime}=J$ y una transición de fase de segundo orden perteneciente a una clase de universalidad de Ising en algún valor finito $\alpha_{2}=J^{\prime} / J$, donde dos mínimos degenerados se fusionan en uno solo. En la siguiente sección mostramos el análisis numérico realizado mediante diagonalización exacta de sistemas pequeños mediante el algoritmo de lanczos (ver sección 2.3.1) con la intención de confirmar los resultados obtenidos hasta el momento.

\subsubsection{Análisis numérico}

En esta sección vamos a explorar el régimen intermedio de $J^{\prime} / J$ no cubierto por la teoría de perturbaciones alrededor de $J^{\prime} / J=0 \mathrm{y}^{\prime} / J=1$, en sistemas finitos por medio de diago- 
nalización exacta de 18 y 24 espines con condiciones de contorno periódicas. Esta parte del trabajo fue realizada en colaboración con el Dr. Marcelo D. Grynberg.

Primero hemos confirmado la presencia del plateau de magnetización en $M=1 / 3$ en todo el rango de acoplamientos desde $J^{\prime} / J=0$ y $J^{\prime} / J=2$. El diagrama de fases magnético 4.13 muestra el campo $h$ necesario para el cruce de niveles entre distintos sectores de magnetización de donde se puede observar lo robusto que es el sector de magnetización $M=1 / 3$. Por
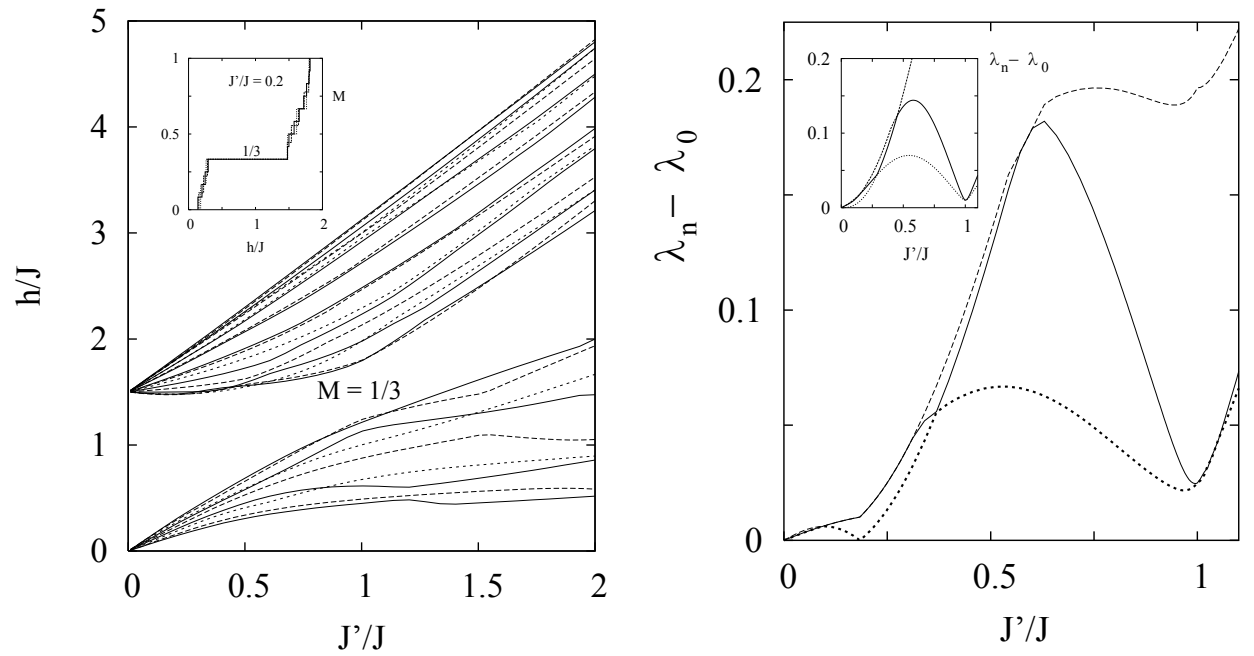

Figura 4.13: (izquierda) Diagrama de fases magnético obtenido por diagonalización exacta de sistemas finitos con 12 (línea punteada), 18 (línea cortada) y 24 espines (línea llena). recuadro: curva de magnetización típica. (derecha) Excitaciones no magnéticas $\lambda_{n}$ para 24 espines con condiciones de contorno periódicas por encima del estado fundamental $\lambda_{0}$, a $M=1 / 3$, para $n=1$ (línea punteada), 2 (línea llena), y 3 (línea cortada). El recuadro muestra los resultados correspondientes para 18 espines.

otro lado, además del estado fundamental hemos calculado las primeras tres excitaciones no magnéticas en el sector $M=1 / 3$ en un amplio rango de acoplamientos $0<J^{\prime} / J<1.5$ y los resultados son mostrados en la figura (4.13). La triple degeneración del estado fundamental es, dentro de los efectos de tamaño finito, cualitativamente observada en el punto $J^{\prime} / J=1$ en acuerdo con referencia [111]. Más aún, asumiendo que el estado fundamental es triplemente degenerado en el limite termodinámico, nuestros datos numéricos son compatibles con el resultado obtenido mediante bosonización, en el cual para $J^{\prime} / J>1$ el estado fundamental es único. De la misma manera, para $J^{\prime} / J \lesssim 1$ la triple degeneración parece ser parcialmente corrida a una doble donde uno de los niveles rápidamente se separa mientras que el otro se mantiene cerca del estado fundamental, y finalmente la degeneración remanente se levanta completamente. Este análisis es consistente con los resultados de bosonización en el limite de modulación débil mostrados en la figura (4.11).

Uno puede estimar la localización de los puntos de las transiciones de fases tipo Ising y BKT mencionadas en la sección 4.2.3 y 4.2.2 por considerar la función- $\beta$ de Callan-Symanzik desarrollada en el contexto del grupo de renormalización fenomenológico por Roomany and 
Wyld [130]. Esta técnica puede ser aplicada en situaciones en la cual la transición de fase no está necesariamente caracterizada por un decaimiento como ley de potencias del gap del espectro, es decir, una ordinaria transición de fase de segundo orden o también una singular de la forma de BKT. En el primer caso la función- $\beta$ se anula mientras que en la otra situación se anula con una singularidad algebraica. Esta función puede se estimada de los datos numéricos para tamaños finitos mediante la aproximación de Roomany-Wyld, que en nuestra notación queda

$$
\begin{aligned}
& \beta_{\mathrm{RW}}(\alpha)=\alpha \ln \left[\frac{N+6}{N} \frac{\Delta_{N+6}(\alpha)}{\Delta_{N}(\alpha)}\right] / \\
& \ln \left(\frac{N+6}{N}\right)\left\{1+\frac{1}{2} \alpha \partial_{\alpha} \ln \left[(N+6) \Delta_{N+6}(\alpha) N \Delta_{N}(\alpha)\right]\right\},
\end{aligned}
$$

donde $\Delta_{N}$ es el gap del espectro por sitio. Notemos que cuando la condición de renormalización fenomenológico $(N+6) \Delta_{N+6}(\alpha)=N \Delta_{N}(\alpha)$ es satisfecha, la función- $\beta$ se anula. Además, su comportamiento es de la forma $\beta(\alpha) \sim\left(\alpha-\alpha_{c}\right) / \nu$, de donde la pendiente en $\alpha_{c}$ está relacionada al exponente de la transición de segundo orden. Por el contrario, en la vecindad de una transición singular de la forma $\Delta \alpha \exp -\left(\alpha-\alpha_{c}\right)^{-\sigma}$, uno tiene $\beta \sim\left(\alpha-\alpha_{c}\right)^{1+\sigma}$ que permite determinar tanto $\alpha_{c}$ como $\sigma$. En la figura 4.14 se muestra la aproximación RW calculada para 18 y 24 espines. La curva de la izquierda caracteriza una transición singular con $\alpha_{1} \sim 0.35$ y $\sigma \sim 0.7$ (aunque no se observa un cero estricto posiblemente debido a efectos de tamaño finito) compatible con la transición BKT, mientras que de la curva de la derecha uno identifica un transición convencional en $\alpha_{2} \sim 0.88$ con $\nu \sim 0.67$ consistente con la clase de Ising.

La transición BKT también puede ser estimada mediante la espectroscópia de cruce de niveles $[131,132,133]$ de los estados de baja energía con diferentes simetrías. En la figura 4.14, se muestran las excitaciones escaleadas $N \Delta E(N)$ como función de $J^{\prime} / J$ para sistemas de $N=18$ y $N=24$ espines. La intersección entre las primer y segunda excitación es interpretada [132] como la transición de fase quiral sin gap-quiral con gap en el punto $\alpha_{1}(N)$ y ocurren en $\alpha=0.29$ y $\alpha=0.36$ respectivamente. El escaleo de tamaño finito de $\alpha_{1}(N)$ es expresado como sigue [131]

$$
\alpha_{1}(N)=\alpha_{1}(\infty)+\text { const } \times N^{-2}
$$

sugiriendo una cruda extrapolación a $\alpha_{1}(\infty) \approx 0.47$.

Estas estimaciones numéricas son consistentes con la existencia de una transición BKT en $\alpha_{1}$ en el rango 0.3-0.5 y una transición tipo Ising en $\alpha_{2}$ alrededor de 0.9. Sin embargo, un cruce de niveles no predicho por el tratamiento analítico, es visto en la figura 4.13 en $J^{\prime} / J \approx 0.18$ en el sistema de 24 espines pero no en el de 18. Su presencia puede ser chequeado en sistemas más grandes, puesto que esta puede ser una consecuencia de términos altamente oscilatorios en la ecuación (4.25) que puede ser determinante en sistemas pequeños. De ser confirmado, esto podría indicar que la transición BKT que separa la fase sin gap descrita en el limite de frustración fuerte de la fase de plateau cuántico en el limite de modulación débil puede se reemplazada por un de primer orden en el limite termodinámico. 

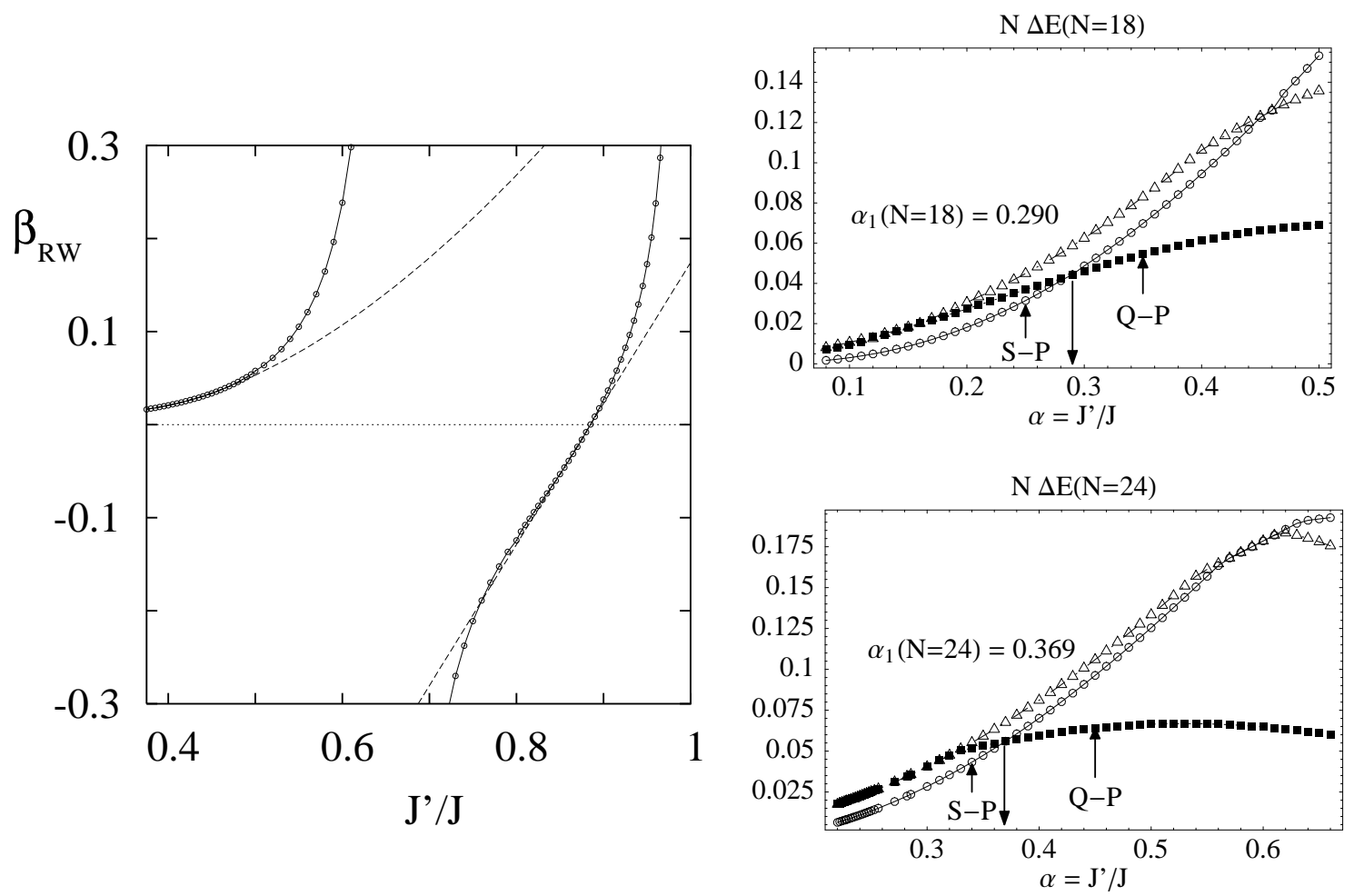

Figura 4.14: (izquierda) Aproximación de Roomany-Wyld $(N=24,18)$ de la función de $\beta$ de Callan-Symanzik (círculos). Las curvas izquierda y derecha son respectivamente con la transición de fase BKT en $\alpha_{1} \approx 0.35$ y con una transición tipo Ising en $\alpha_{2} \approx 0.88$. Las curvas entrecortadas ajustan los datos numéricos con parámetros referidos en el texto. (derecha) Estados excitados y cruce de niveles usados en el análisis. 
Para caracterizar las fases separadas por la mencionada transición, podemos calcular los perfiles de magnetización del estado fundamental. Encontramos tres diferentes fases periódicas dependiendo del valor de $J^{\prime} / J$ Como el perfil es periódico, solo mostramos la configuración genérica de un triángulo, etiquetando los sitios $a=1,2,3$ en acuerdo con la figura 4.7. Un gráfico de los perfiles locales de magnetización del estado fundamental para $J^{\prime} / J$ hasta 1.4 se muestra en la figura 4.15 (panel superior). Notemos que los sitios $a=2,3$ muestran la misma magnetización.

En el limite de frustración fuerte, observamos un perfil de magnetización uniforme para $0<J^{\prime} / J \lesssim 0.18$ (por ejemplo $S^{z 1,2,3}=0.018,0.016,0.016$ en $J^{\prime} / J=0.1$ ). En contraste, para $0.18<J^{\prime} / J \lesssim 1$ encontramos una fase tipo plateau cuántico (el: $S^{z 1,2,3}=$ $0.470,0.015,0.015$ en $\left.J^{\prime} / J=0.5\right)$ y finalmente para $J^{\prime} / J \gtrsim 1$ una fase tipo up-up-down (ej: $S^{z 1,2,3}=-0.075,0.288,0.288$ en $J^{\prime} / J=1.2$ ). Encontramos claros signos de un cruce de niveles en $J^{\prime} / J=1$.

Uno puede apreciar un cambio repentino en la magnetización en el punto del cruce de niveles $J^{\prime} / J=0.18$; magnetización uniforme para $0<J^{\prime} / J \lesssim 0.18\left(S^{z} \approx 0.166\right.$ en cada sitio) y fase de plateau cuántico para $0.18<J^{\prime} / J$. Los resultados numéricos pueden resumirse por el parámetro de orden

$$
M_{s}=\sum_{i} \cos \left(\frac{2 \pi}{3}(i-2)\right)<S_{i}^{z}>
$$

En la figura 4.15 (panel inferior), este parámetro permite identificar las tres fases mencionadas anteriormente. Notemos que al estar caracterizadas por fluctuaciones de largo alcance, las transiciones de segundo orden son particularmente difíciles de detectar en sistemas de tamaño finito.

\subsubsection{Resultados y discusión}

En esta sección hemos analizado las transiciones de fase cuánticas en una cadena trimerizada a magnetización a magnetización $M=1 / 3$. Hemos encontrado que la triple degeneración del estado fundamental en el plateau 1/3 en el caso de la cadena homogénea es levantada dando lugar a varias fases magnéticas separadas por transiciones de primer y segundo orden. El análisis numérico mediante diagonalización exacta de sistemas pequeños confirman los resultados analíticos. Otra transición separa el régimen de modulación débil del régimen de frustración fuerte. Partiendo del límite de acoplamiento débil hasta más allá del punto homegémeo, el diagrama de fases del estado fundamental es esquemáticamente mostrado en la figura (4.6) muestra: una fase con magnetización uniforme descrita por excitaciones quirales no magnéticas sin gap; luego una transición a un estado fundamental no degenerado tipo plateau cuántico; luego una transición de segundo orden, en la clase de universalidad de Ising, a un estado fundamental doblemente degenerado y finalmente una transición de primer orden en el punto homogéneo a un estado fundamental tipo $\uparrow \uparrow \downarrow$. La naturaleza de la transición entre la fase quiral y el plateau cuántico no es posible determinar con nuestros datos numéricos; esta zona es dejada en blaco en el diagrama como un problema abierto. 
100 Fases tipo espín-Peierls en cadenas de espines a $M=1 / 3$.

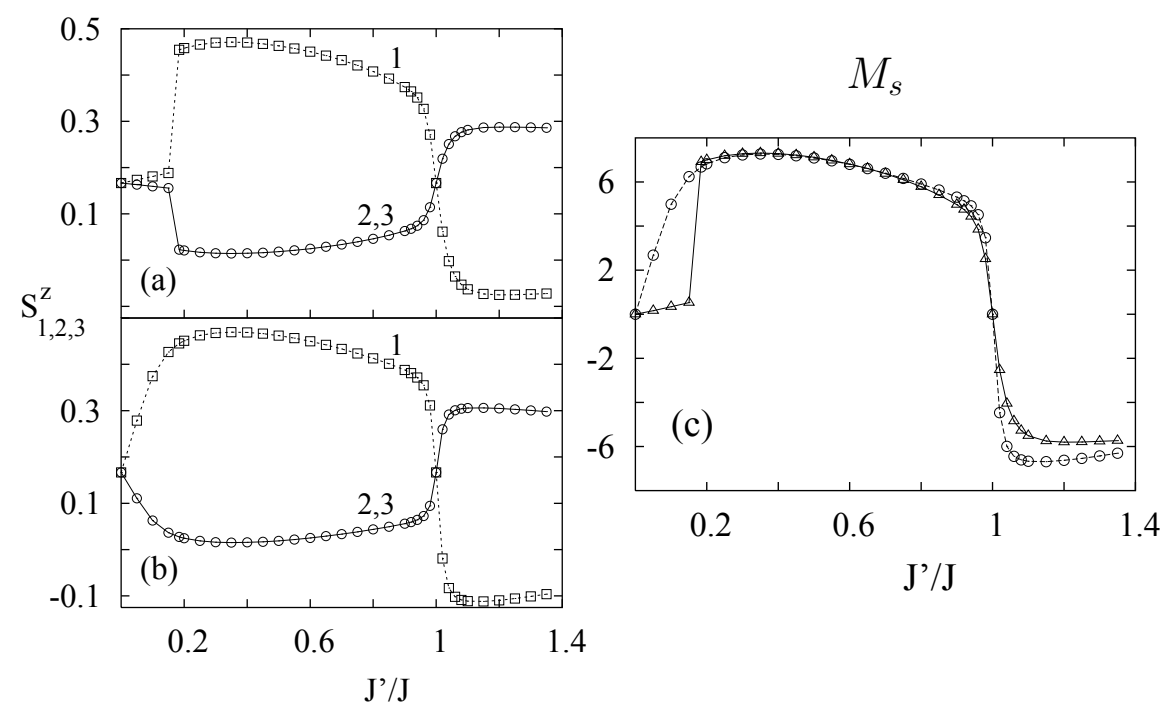

Figura 4.15: (a): magnetización local del estado fundamental para 24 sitios como función del parámetro de acoplamiento. Los cuadrados (círculos) corresponden al sitio medio (1)(extremos (2), (3)) de los trímeros. El panel (b) muestra respectivamente estos resultados para 18 espines (aquí, la fase uniforme está ausente). (c): parámetro de orden en ecuación (4.32) describiendo magnetizaciones moduladas para 18 (círculos) y 24 (triángulos) espines. De izquierda a derecha éste exhibe los estados de magnetización uniforme, plateau cuántico y $\uparrow \uparrow \downarrow$.

\subsection{Fases tipo espín-Peierls en cadenas de espines a $M=1 / 3$.}

Continuando con el estudio previo de sistemas magnéticos a $M=1 / 3$, vamos a investigar la posibilidad de que deformaciones elásticas de cadenas antiferromagnéticas de espín $S=1 / 2$ a magnetización $M=1 / 3$ produzcan modulaciones en los acoplamientos magnéticos similares a las consideradas en la sección 4.2, mediante el mecanismo conocido como espínPeierls. En particular vamos a estudiar las deformaciones posibles de la red acoplada a los grados de libertad de espines en el límite adiabático. Para llevar a cabo este estudio, utilizamos bosonización abeliana y diagonalización exacta de sistemas pequeños. Ambos métodos predicen la existencia de fases magneto-elásticas dependiendo del grado de frustración en el sistema.

\subsubsection{Introducción}

Los sistemas de espines han sido continuamente explorados en los últimos años. Frustración magnética es considerado como un ingrediente fundamental para inducir órdenes magnéticos poco convencionales o incluso fases desordenadas con excitaciones exóticas. Co- 
mo representativo de cadenas homogéneas geométricamente frustradas, uno puede citar la cadena zig-zag antiferromagnética de espín $S=1 / 2$ realizada en compuestos como: $\mathrm{CuGeO}_{3}$ [134], $\mathrm{LiV}_{2} \mathrm{O}_{5}$ [135] o $\mathrm{SrCuO}_{2}$ [136].

En este contexto, tanto los estudios experimentales como teóricos han crecido debido al descubrimiento del compuesto $\mathrm{CuGeO}_{3}$ que presenta una transición de espín-Peierls a magnetización cero [38]. Esta transición es una inestabilidad debida al acoplamiento de la red con los grados de libertad de espín, y que se caracteriza (por debajo de la temperatura crítica $\left.T_{S P}\right)$ por abrir un gap de espín en el espectro de excitaciones y la aparición de una distorsiones dimerizada a $M=0$, con la consecuencia de una modulación en los acoplamientos magnéticos (ver 4.16). Un fenómeno similar puede ser analizado en sistemas magneto-elásti-

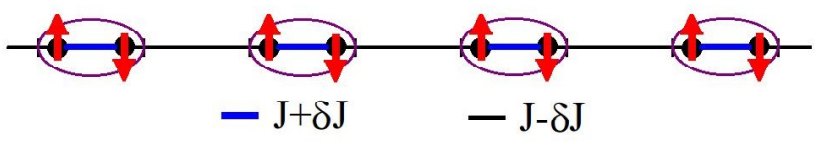

Figura 4.16: Esquema de una fase de espín dimerizada.

cos a magnetización no nula, siendo los más interesantes los sistemas que presentan plateaux de magnetización. Más aún, recientemente se ha mostrado que la interacción espín-fonón en cadenas zig-zag produce una la apertura de un gap de espín en el espectro de excitaciones, así como la presencia de plateaux de magnetización en el límite de baja frustración [118]. Tales plateaux de magnetización se deben a un mecanismo de conmensurabilidad entre distorsiones de la red y modulaciones de espín.

Con respecto a la situación de cadenas no elásticas a magnetización $M=1 / 3$, en la sección anterior mostramos que pequeñas modulaciones de los acoplamientos magnéticos con período tres pueden conducir a la transiciones magnéticas desde un estado fundamental tres veces degenerado hacia uno de los siguientes dos estados: uno de ellos conocido como plateau clásico, donde la configuración de espines se asimila a un estado tipo Ising de la forma $\uparrow \uparrow \downarrow$, o bien hacia el estado conocido como plateau cuántico cuya configuración es de la forma $\bullet \bullet \uparrow$ (singlete-espin-up). Recientemente se han realizado estudios tanto experimentales como teóricos de sistemas que presentan plateaux cuánticos a magnetización $M=1 / 3$ para el compuesto $\mathrm{Cu}_{3}\left(\mathrm{P}_{2} \mathrm{O}_{6} \mathrm{OH}\right)_{2}$ [137] que es bien descrito por un modelo de Heisenberg antiferromagnético con $S=1 / 2$ y acoplamientos magnéticos modulados de período tres.

Una idea acerca del estado fundamental magneto-elástico puede ser obtenida a partir de la modulación mencionada a $M=1 / 3$. Cuando uno considera una cadena $J_{1}-J_{2}$ con acoplamientos con la red solo a primeros vecinos, una deformación de la red en la cual se acercan dos sitios vecinos a un mismo sitio (ver figura 4.17 panel superior) induce una modulación en los acoplamientos $J_{1}$ formando trímeros. El caso límite de un trímero aislado, con $S_{z}^{\text {total }}=1 / 2$ indica [93] que el estado fundamental corresponde a una de las configuraciones $\uparrow \downarrow \uparrow$ en cada trímero. Sin embargo, si por el contrario, dos de cada tres sitios se agrupan juntos formando dímeros (ver figura 4.17 panel inferior) el caso límite de dímeros aislados y sitios intercalados, el estado fundamental corresponde a $\bullet \uparrow$, formados por un singlete en cada dímero. 
(a)

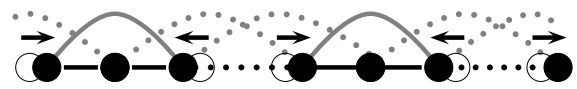

(b)

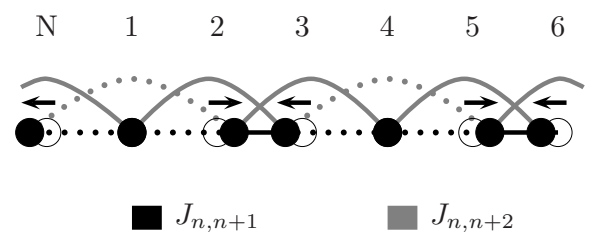

Figura 4.17: El panel superior describe la deformación de la red que forma trímeros; panel inferior la deformación permite la formación de dímeros.

Motivados por la discusión anterior, en esta sección estudiamos las posibles fases magnetoelásticas en el modelo de Heisenberg antiferromagnético $J_{1}-J_{2}$ a magnetización $M=1 / 3$. En particular investigamos el sistema incluyendo acoplamiento espín-fonón tanto a primeros como a segundos vecinos en el límite adiabático. Mostramos que el estado fundamental (a temperatura $T=0$ ) favorece patrones de distorsión de la red, derivados a partir de la competición entre energía elástica y magnética. Estos patrones rompen espontáneamente la simetría de traslación, con diferentes fases dependiendo de la relación entre el acoplamiento a primeros y segundos vecinos $J_{2} / J_{1}$, y del valor del acoplamiento espín-fonón. Como sugiere la figura 4.17, el sistema presenta esencialmente dos situaciones diferentes: (i) una tendiendo a agrupar tres sitios consecutivos para formar trímeros y (ii) otra tendiendo a agrupar dos de cada tres sitios para formar dímeros. Para cada patrón de deformación, dependiendo de los parámetros microscópicos, el sector de espín adopta ya sea: (a) una configuración de plateau clásica, o bien (b) una configuración de plateau cuántica.

\subsubsection{Descripción del modelo y bosonización}

Para comenzar, consideremos el hamiltoniano de Heisenberg de $S=1 / 2$ con acoplamientos a primeros y segundos vecinos,

$$
\mathcal{H}_{M}=\sum_{n}\left(J_{n, n+1} \mathbf{S}_{n} \cdot \mathbf{S}_{n+1}+J_{n, n+2} \mathbf{S}_{n} \cdot \mathbf{S}_{n+2}-h S_{n}^{z}\right) .
$$

La interacción de los espines en una cadena homogénea $\left(J_{n, n+1}=J_{1}, J_{n, n+2}=J_{2}\right)$ con los fonones se obtiene expandiendo los acoplamientos magnéticos alrededor de los valores no distorsionados $J_{1}$ y $J_{2}$ (ver sección 1.4) dando como resultado

$$
\begin{aligned}
& J_{n, n+1} \approx J_{1}\left(1-A\left(u_{n+1}-u_{n}\right)\right), \\
& J_{n, n+2} \approx J_{2}\left(1-B\left(u_{n+2}-u_{n}\right)\right),
\end{aligned}
$$

donde $u_{n}$ es coordenada escalar relevante para el desplazamiento del ión $n$ respecto de la posición de equilibrio, mientras que $A$ y $B$ son las constantes de acoplamiento espín-fonón a 
primeros y segundos vecinos respectivamente. El hamiltoniano total, incluyendo la energía elástica en la aproximación adiabática, está dado por

$$
\begin{aligned}
H_{T}= & \frac{1}{2} K \sum_{n}\left(u_{n+1}-u_{n}\right)^{2}+ \\
& +\sum_{n}\left\{J_{1} \mathbf{S}_{n} \cdot \mathbf{S}_{n+1}+J_{2} \mathbf{S}_{n} \cdot \mathbf{S}_{n+2}\right\}- \\
& -\sum_{n}\left\{J_{1} A\left(u_{n+1}-u_{n}\right) \mathbf{S}_{n} \cdot \mathbf{S}_{n+1}+\right. \\
& \left.+J_{2} B\left(u_{n+2}-u_{n}\right) \mathbf{S}_{n} \cdot \mathbf{S}_{n+2}\right\},
\end{aligned}
$$

donde $K$ es la constante elástica. En la ecuación (4.35) la primera línea corresponde la energía elástica de los fonones clásicos $\left(H_{C P}\right)$; la segunda línea al hamiltoniano homogéneo magnético $\left(H_{M}\right)$ y el resto a la interacción espín-fonón $\left(H_{I}\right)$,

$$
H_{T}=H_{C P}+H_{M}+H_{I}
$$

Por conveniencia para el análisis numérico posterior, adimensionalizamos los parámetros usando $J_{1}$ como escala de energía como sigue:

$$
\begin{aligned}
A & \rightarrow \tilde{A}=\left(J_{1} / K\right)^{1 / 2} A \\
B & \rightarrow \tilde{B}=\left(J_{1} / K\right)^{1 / 2} B \\
u_{n} & \rightarrow \tilde{u}_{n}=\left(K / J_{1}\right)^{1 / 2} u_{n} \\
J_{1}, J_{2} & \rightarrow \alpha=J_{2} / J_{1}
\end{aligned}
$$

Para estudiar semi-cuantitativamente las propiedades de baja energía del modelo (4.35), empleamos el método de bosonización abeliana desarrollado en la sección 2.1. Para llevar a cabo esto, comenzamos con el hamiltoniano magnético $H_{M}$ y seguimos los mismos pasos desarrollados en la sección sección 2.1.4. Primero mediante la transformación de Jordan-Wigner (ver sección 2.1.4) introducimos fermiones sin espín $\psi_{n}$; entonces uno escribe una aproximación lineal alrededor del nivel de Fermi (a magnetización $M=1 / 3, k_{F}=\pi / 3 a$ donde $a$ es el espaciamiento de la red) de estos fermiones en términos de fermiones "izquierdos " y "derechos"

$$
\psi_{n} \approx e^{i \pi n / 3} \psi_{R}(n a)+e^{-i \pi n / 3} \psi_{L}(n a) .
$$

Luego de pasar al continuo introduciendo la coordenada continua $x=n a$ y aplicando las reglas de bosonización, el hamiltoniano $H_{M}$ es finalmente mapeado en un hamiltoniano gaussiano

$$
\frac{v}{2} \int d x\left[\frac{1}{K_{L}}(\partial \varphi)^{2}+K_{L}(\partial \theta)^{2}\right]
$$

En la ecuación (4.39), $\varphi$ es un bosón compactificado definido en un círculo, $\varphi \equiv \varphi+\sqrt{\pi}$, y $\theta$ es su campo dual definido por $\partial_{x} \theta=\partial_{t} \varphi$. Los parámetros $v$ y $K_{L}$ (velocidad de Fermi y 
parámetro de Tomonaga-Luttinger respectivamente) dependen de los parámetros microscópicos del hamiltoniano $H_{M}$; $v$ es proporcional a $a J_{1}$, mientras que $K_{L}$ es adimensional.

Un comportamiento particular que ocurre a $M=1 / 3$ y que hemos mencionado en la sección 4.2.3, es que el momento de Fermi $k_{F}=\pi / 3 a$ permite que el proceso triple Umklapp [93] se vuelva conmensurado, dando como resultado, una perturbación al término gaussiano dado por

$$
-\frac{g_{3} v}{2 \pi^{2} a^{2}} \int d x \cos (3 \sqrt{4 \pi} \varphi)
$$

donde el coeficiente $g_{3}$ es no universal. Como mencionamos en la sección 4.1 el hamiltoniano magnético homogéneo $H_{M}$ describe una fase tipo Tomonaga-Luttinger (TL) para $0<J_{2} / J_{1}<\alpha_{c}=0.56$. En la región $\alpha_{c}<J_{2} / J_{1} \lesssim 1.25$ existe un plateau en la curva de magnetización en $M=1 / 3$. En términos de los parámetros de la bosonización, para que ocurra esto debe ser $K_{L}>2 / 9$ para $J_{2} / J_{1} \lesssim \alpha_{c}$, ya que esto implica que la perturbación en Eq. (4.40) sea irrelevante. Entonces el coeficiente $g_{3}$ "fluye" a cero al aplicar el grupo de renormalización y la teoría efectiva describe una fase TL. Por otro lado, para $\alpha_{c} \lesssim J_{2} / J_{1} \lesssim 1.25$, debe ser $K_{L}<2 / 9$, haciendo (4.40) una perturbación relevante. Entonces éste término abre un gap en el espectro de excitaciones magnéticas generando un plateau en la curva de magnetización. Más aún, en la región con gap la simetría de traslación es espontáneamente rota a una configuración de la forma $\uparrow, \uparrow, \downarrow$. Tales configuraciones son descritas al fijar el campo bosónico en uno de los mínimos de la ecuación (4.40) considerado como la energía potencial semiclásica [46], siempre que $g_{3}>0$.

En consecuencia, representamos cualitativamente el plateau de magnetización por el comportamiento del coeficiente no universal $g_{3} \geq 0$ siendo éste no nulo para $\alpha_{c} \lesssim J_{2} / J_{1} \lesssim 1.25$, con un máximo en algún punto intermedio de $J_{2} / J_{1}$. Como aclaración, nosotros no estudiamos la región $J_{2} / J_{1}>1.25$, donde el plateau de magnetización $M=1 / 3$ no está presente; ésta región puede se analizada mejor proponiendo como punto da partida dos cadenas de espines con acoplamiento $J_{2}$, débilmente acopladas por la interacción $J_{1}$. Ahora consideremos las deformaciones de la red. Partiendo del conocimiento del estado fundamental del caso homogéneo $J_{1}-J_{2}$ a $M=1 / 3$ con $J_{2} / J_{1}>\alpha_{c}$, uno puede argumentar que la deformación adiabática de la red causada por el acoplamiento espín-fonón en la ecuación (4.35) tendrá período tres. Éste "ansatz" es soportado por bosonización, siempre que la deformación sea conmensurada con $k_{F}=\pi / 3 a$.

La deformación más general de período tres (ver apéndice A) está dada por

$$
u_{n}=\frac{u_{0}}{\sqrt{3}} \sin \left(\frac{2 \pi}{3} n-\chi\right)
$$

con amplitud $u_{0} \mathrm{y}$ una fase relativa $\chi$ como parámetros libres. Nuestro propósito es buscar la deformación que minimiza la energía magneto-elástica. Una vez que un mínimo es encontrado, $u_{0}$ indicará la amplitud de la deformación y la fase $\chi$ relacionará el patrón de la deformación al correspondiente estado fundamental del sector de espín caracterizado por el valor de $\varphi$ en el mínimo del potencial.

De la ecuación (4.41), la distorsión de los acoplamientos a primeros vecinos entre los sitios 
$n$ y $n+1$, denotado por $\delta_{n}=u_{n+1}-u_{n}$ queda

$$
\delta_{n}=u_{0} \cos \left(\frac{2 \pi}{3}\left(n+\frac{1}{2}\right)-\chi\right),
$$

mientras que la distorsión a segundos vecinos es

$$
\delta_{n+1}+\delta_{n}=u_{0} \cos \left(\frac{2 \pi}{3}(n+1)-\chi\right) .
$$

La energía elástica asociada a las deformaciones en (4.41) es

$$
H_{C P} / J_{1}=\frac{1}{4} N \tilde{u}_{0}^{2} .
$$

Finalmente consideremos el término de interacción espín-fonón $H_{I}$ inducido por las deformaciones de la red en la ecuación (4.41). Siguiendo el procedimiento de bosonización, se genera el siguiente término

$$
\frac{\tilde{u}_{0} v}{2 \pi^{2} a^{2}} \int d x\left(f_{1} \cos (\sqrt{4 \pi} \varphi+\chi)+f_{2} \cos (2 \sqrt{4 \pi} \varphi-\chi)\right)
$$

que introduce un primer y segundo armónico del campo bosónico con coeficiente proporcional a la amplitud de la deformación $\tilde{u}_{0}$. Notemos que estos operadores son más relevantes que el tercer armónico en la ecuación (4.40), y por lo tanto deben ser tenidos en cuenta tanto en el régimen $J_{2} / J_{1}>\alpha_{c}$ como para $J_{2} / J_{1}<\alpha_{c}$, siempre que $K_{L}<1 / 2$. Más allá que los coeficientes son no universales, es muy útil notar que al más bajo orden perturbativo se tiene

$$
\begin{aligned}
& f_{1} \sim \tilde{A}\left(1-C_{1} J_{2} / J_{1}\right) \\
& f_{2} \sim-\tilde{A}\left(1+C_{2} q J_{2} / J_{1}\right),
\end{aligned}
$$

donde $q=\tilde{B} / \tilde{A}$ y $C_{1}, C_{2}$ son constantes positivas con $C_{2} \ll C_{1}$.

Para una descripción cualitativa, vamos a asumir que $f_{1}$ y $f_{2}$ dependen de los parámetros microscópicos de la manera sugerida por esta ecuación.

Juntando todo, podemos escribir la teoría efectiva como

$$
H_{T}=H_{C P}+H_{\text {free }}+V_{\text {eff }}
$$

donde $H_{C P}$ es la contribución a la energía elástica dada en la ecuación (4.44),

$$
H_{\text {free }}=\frac{v}{2} \int d x\left[\frac{1}{K_{L}}(\partial \varphi)^{2}+K_{L}(\partial \theta)^{2}\right]
$$

es la parte gaussiana del hamiltoniano bosónico y

$$
\begin{aligned}
V_{\text {eff }}= & \frac{v}{2 \pi^{2} a^{2}} \int d x\left[\tilde{u}_{0} f_{1} \cos (\sqrt{4 \pi} \varphi+\chi)\right. \\
& \left.+\tilde{u}_{0} f_{2} \cos (2 \sqrt{4 \pi} \varphi-\chi)-g_{3} \cos (3 \sqrt{4 \pi} \varphi)\right]
\end{aligned}
$$

es el potencial de autointeracción que define una teoría denominada "triple seno-Gordon" [127]. Para nuestro propósito será suficiente realizar un tratamiento semi-clásico análogo al realizado en capítulo 3, y en el presente capítulo sección 4.2.3. 


\subsubsection{Análisis semiclásico de la teoría efectiva}

Como mencionamos anteriormente, el objetivo consiste es buscar las posibles deformaciones elásticas de minimizan la energía magneto-elástica con respecto al caso homogéneo no deformado. Dentro de esta aproximación semiclásica la energía por sitio depende de tres parámetros que definen la configuración, $\tilde{u}_{0}, \varphi$ y $\chi$, y es fácilmente evaluable a

$$
\begin{aligned}
& \epsilon\left(\tilde{u}_{0}, \varphi, \chi\right) \equiv \frac{E}{J_{1} N}=\frac{1}{4} \tilde{u}_{0}^{2}-\frac{g_{3}}{2 \pi^{2}} \cos (3 \sqrt{4 \pi} \varphi) \\
& \quad+\frac{\tilde{u}_{0}}{2 \pi^{2}}\left(f_{1} \cos (\sqrt{4 \pi} \varphi+\chi)+f_{2} \cos (2 \sqrt{4 \pi} \varphi-\chi)\right),
\end{aligned}
$$

tal que el mínimo puede ser encontrado analíticamente. Notemos que esta expresión es invariante bajo los corrimientos simultáneos

$$
\begin{aligned}
\sqrt{4 \pi} \varphi & \rightarrow \sqrt{4 \pi} \varphi+2 \pi / 3 \\
\chi & \rightarrow \chi-2 \pi / 3
\end{aligned}
$$

en relación con la estructura de período tres de la cadena. Esto permite restringir el análisis a $0<\sqrt{4 \pi} \varphi \leq 2 \pi / 3$ sin perder generalidad. También un corrimiento de $\chi \rightarrow \chi+\pi$ es equivalente a cambiar el signo de $u_{0}$, permitiendo considerar $0 \leq \chi<\pi$.

La energía que minimiza el potencial efectivo es siempre encontrado en una de las siguientes situaciones:

1. $\sqrt{4 \pi} \varphi=2 \pi / 3, \chi=\pi / 3, \tilde{u}_{0}=\left(f_{1}+f_{2}\right) / \pi^{2}$, donde la energía es evaluada a

$$
\epsilon=-\frac{g_{3}}{2 \pi^{2}}-\frac{\left(f_{1}+f_{2}\right)^{2}}{4 \pi^{4}} .
$$

2. $\sqrt{4 \pi} \varphi=\pi / 3, \chi=2 \pi / 3, \tilde{u}_{0}=\left(f_{1}-f_{2}\right) / \pi^{2}$, donde la energía es evaluada a

$$
\epsilon=\frac{g_{3}}{2 \pi^{2}}-\frac{\left(f_{1}-f_{2}\right)^{2}}{4 \pi^{4}} .
$$

Antes de mostrar el diagrama de fases resultante, vamos a discutir el contenido físico de las posibles fases. Siguiendo las reglas usuales de bosonización para mapear $\varphi$ a variables de espín [45], el valor $\sqrt{4 \pi} \varphi=2 \pi / 3$ en la primera solución indica que el sector de espín adopta una configuración tipo plateau clásico (que llamaremos $C P$ ), que corresponde a seleccionar uno de los tres estados fundamentales del tipo $\uparrow \uparrow \downarrow$. Vamos a llamar a su energía $\epsilon_{C P}$. La fase relativa $\chi=\pi / 3$ y el signo de $\tilde{u}_{0}$ determinan la deformación elástica. Para $f_{1}+f_{2}>0$ uno encuentra una deformación que agrupa bloques de tres espines en trímeros $(T$, ver figura 4.17, panel superior); en el caso opuesto se forman grupos de dos espines formando dímeros, alternando dos espines cerca con dos espines más separados ( $D$, ver figura 4.17 , panel inferior). En la segunda solución, el valor $\sqrt{4 \pi} \varphi=\pi / 3$ no es uno de los mínimos del potencial efectivo para el caso homogéneo, es decir, que corresponde a una solución que surge 
debido a las deformaciones e indica además que el sector de espín adopta una configuración tipo plateau cuántico $\bullet \uparrow[93] Q P$. La correspondiente energía la llamamos $\epsilon_{Q P}$. La fase relativa $\chi=2 \pi / 3$ en esta solución indica que la deformación de la red forma dímeros $(D)$ para $f_{1}-f_{2}>0$ y trímeros $(T)$ en el otro caso. Dependiendo de los coeficientes $f_{1}, f_{2}$ y $g_{3}$, que a su vez dependen de los parámetros microscópicos, una de estas soluciones será seleccionada como un mínimo global y determina la fase magneto-elástica del estado fundamental. Para presentar un diagrama de fases esquemático, asumimos que la dependencia fenomenológica de $f_{1}, f_{2}$ y $g_{3}$ en los parámetros microscópicos detallados en la sección previa. Siguiendo la referencia [138] hemos escogido una relación $\tilde{B}=1.5 \tilde{A}$, que es usada en el resto del análisis, como representativo de materiales donde el acoplamiento espín-fonón entre segundos vecinos juega una rol importante. Finalmente, las fases magneto-elásticas "son mostradas" en la figura 4.18 .

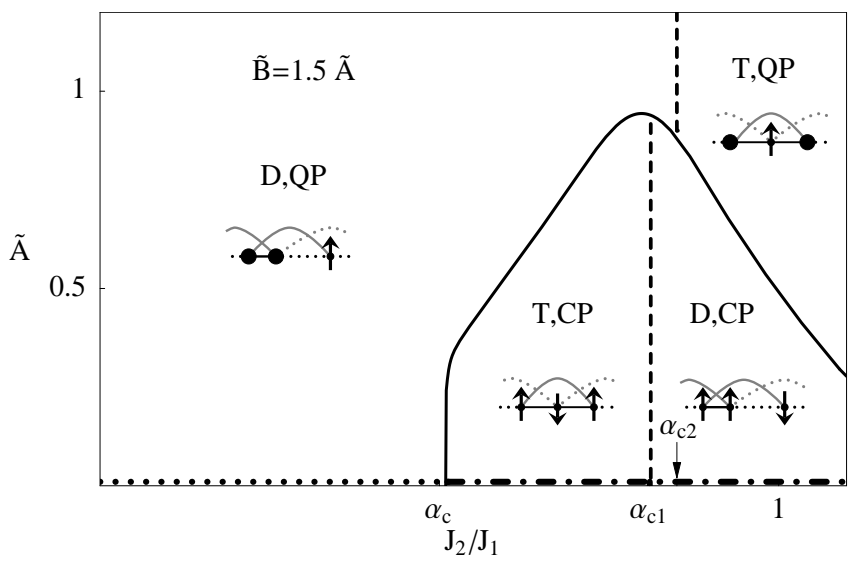

Figura 4.18: Diagrama de fases magneto-elástico con acoplamientos espín-fonón a primeros y segundos vecinos relacionados por $\tilde{B}=1.5 \tilde{A}$. Las fases del estado fundamental tipo $\uparrow \uparrow \downarrow$ y plateau cuántico son identificadas por $C P$ y $Q P$ respectivamente. Las fases elásticas con dímeros y trímeros son identificadas por $D$ y $T$. Los patrones magneto-elásticos en cada fase son mostrados por diagramas esquemáticos.

Para comprender mejor el como obtuvimos el diagrama 4.18, en la figura 4.19 mostramos la evolución de la energías $\epsilon_{C P}, \epsilon_{Q P}$ como función del acoplamiento a primeros vecinos $\tilde{A}$, $\tilde{B}=1.5 \tilde{A}$, fijando $J_{2} / J_{1}=0.7$. El cruce de energías en $\tilde{A}_{c} \simeq 0.65$ muestra una transición de desde la fase magnética $C P$ a la $Q P$. Cabe aclarar que la transición que observamos es muy diferente a la que hemos observado en la sección 4.2.1, donde el sistema pasa de la fase $C P$ a la fase $Z_{2}$ y posteriormente a la fase $Q P$ a través de una transición de fase de segundo orden.

Dentro de cada fase magnética se pueden identificar las diferentes fases elásticas. Dado $\tilde{A}$, encontramos los valores críticos de $J_{2} / J_{1}$ donde $\tilde{u}_{0}$ cambia de signo: en la fase $C P$, la ecuación $f_{1}\left(\alpha_{c 1}\right)=-f_{2}\left(\alpha_{c 1}\right)$ define una línea crítica $J_{2} / J_{1}=\alpha_{c 1}$ tal que para $J_{2} / J_{1}<\alpha_{c 1}$ el sistema adopta una distorsión de la red forman trímeros $T$ mientras que para $J_{2} / J_{1}>\alpha_{c 1}$ la deformación agrupa dímeros $D$. En contraste, en la fase $Q P$ encontramos una línea crítica $\alpha_{c 2}$ donde $f_{1}\left(\alpha_{c 2}\right)=f_{2}\left(\alpha_{c 2}\right)$, siendo la distorsión del tipo $D$ para $J_{2} / J_{1}<\alpha_{c 2}$ y tipo $T$ para 


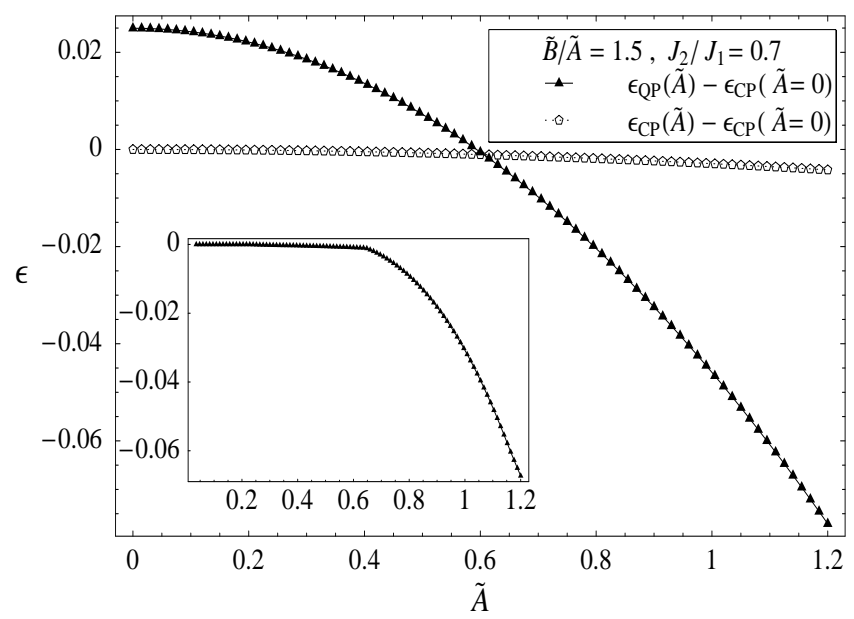

Figura 4.19: Energías semiclásicas para el mínimo de ambos tipos de fases (plateau clásico y cuántico) en términos del acoplamiento espín-fonón $\tilde{A}$, para $\tilde{B}=1.5 \tilde{A}$ y $J_{2} / J_{1}=0.7$. El recuadro muestra la energía del estado fundamental obtenida mediante diagonalización exacta de un sistema de 24 sitios, después de fijar el cero de referencia.

$J_{2} / J_{1}>\alpha_{c 2}$. Usando las expresiones para $f_{1}, f_{2}$ mostradas en (4.46), estas líneas críticas no dependen de $\tilde{A}$.

Un análisis similar puede ser hecho para $J_{2} / J_{1} \lesssim \alpha_{c}$. La diferencia más importante es que en está región el parámetro de Tomonaga-Luttinger es $K_{L}>2 / 9$ y el tercer armónico es irrelevante. Representamos esta situación fijando $g_{3}=0$. A diferencia del caso previo, no hay cruce entre $\epsilon_{Q P}$ y $\epsilon_{C P}$; el mínimo absoluto de la energía siempre corresponde a $\epsilon_{Q P}$, seleccionando la fase magnética $Q P$. También encontramos $\left(f_{1}-f_{2}\right)>0$ en la región completa, de modo que la fase corresponde a la tipo $D$.

La posición relativa de las deformaciones elásticas y los perfiles de magnetización en cada fase es determinada por los correspondientes valores de $\varphi$ y $\chi$, como puede ser encontrado usando la ecuación (4.41) y las formulas de bosonización.

Los diferentes valores de $\tilde{B} / \tilde{A}$ pueden ser analizados de manera similar. Lo que hemos observado, es que al disminuir su valor se produce un incremento en la región caracterizada por el plateau clásico y las deformaciones trimerizadas, con valores más altos de ambos $\alpha_{c 1}$ y $\tilde{A}_{c}$.

Es importante notar que la amplitud de la deformación $\tilde{u}_{0}$ es proporcional a $f_{1}, f_{2}$, que a su vez son proporcionales a los acoplamientos adimensionales $\tilde{A}, \tilde{B}$. En la figura 4.18, el patrón elástico evoluciona hacia el límite homogéneo a medida que $\tilde{A} \rightarrow 0$. Por construcción, la teoría efectiva en las ecuaciones (4.48), (4.49) describe en este límite una fase correspondiente a un líquido de Luttinger para $J_{2} / J_{1}<\alpha_{c}$ y una teoría tipo seno-Gordon con un estado fundamental triplemente degenerado para $J_{2} / J_{1}>\alpha_{c}$.

Es interesante mencionar que si nuestra aproximación permanece válida en el límite $J_{2} / J_{1} \rightarrow$ 0, describiendo una cadena de espines con interacciones solo a primeros vecinos, el sistema 
adopta una deformación elástica trimerizada cuyo patrón sería: dos enlaces débiles y uno fuerte (ver figura 4.17, panel inferior). Este modelo fue recientemente estudiado numéricamente con Montecarlo Cuántico motivados por el compuesto $\mathrm{Cu}_{3}\left(\mathrm{P}_{2} \mathrm{O}_{6} \mathrm{OH}\right)_{2}$ [137], encontrando precisamente una fase de plateau cuántico.

\subsubsection{Análisis numérico}

En esta sección mostramos los resultados del análisis numérico que realizamos del hamiltoniano (4.35) mediante diagonalización exacta de sistemas pequeños, con el objetivo contrastar los resultados mostrados en la sección previa. Dicho análisis se llevo a cabo utilizando el algoritmo de Lanczos para sistemas de hasta $N=24$ sitios con condiciones de contorno periódicas ${ }^{2}$.

La estrategia fue la siguiente: las deformaciones de período tres son parametrizadas por dos distorsiones independientes, digamos $\delta_{1}=u_{2}-u_{1} \mathrm{y} \delta_{2}=u_{3}-u_{2}$, mientras que $\delta_{3}=-\delta_{1}-\delta_{2}$ y $\delta_{n+3}=\delta_{n}$. Para cada valor de $J_{2} / J_{1}$ y $\tilde{A}$, fijando $\tilde{B} / \tilde{A}$ y $M=1 / 3$, calculamos el estado fundamental del hamiltoniano (4.35) para un amplio rango de deformaciones elásticas $\left(\delta_{1}, \delta_{2}, \delta_{3}\right)$ y entonces seleccionamos el mínimo absoluto.

Encontramos que, en acuerdo con los resultados de la bosonización, la configuración con la energía más baja es siempre obtenida a una de las distorsiones mostradas en la figura 4.17:

1. $\left(\delta_{1}, \delta_{2}, \delta_{3}\right)=\left(-\frac{1}{2} \Delta,-\frac{1}{2} \Delta, \Delta\right)$ que corresponde a una fase de trímeros $(T)$, o

2. $\left(\delta_{1}, \delta_{2}, \delta_{3}\right)=\left(\frac{1}{2} \Delta, \frac{1}{2} \Delta,-\Delta\right)$ que corresponde a una fase de dímeros $(D)$.

Para poder caracterizar las fases magnéticas, calculamos la magnetización local $\left\langle S_{n}^{z}\right\rangle$ del estado fundamental. El parámetro de orden

$$
M_{S}=\frac{1}{N} \sum_{n} \cos \left(\frac{2 \pi}{3}(n-2)\right)<S_{n}^{z}>
$$

es positivo en la fase correspondiente a el plateau cuántico (QP) y negativo en el plateau clásico (CP). Realizamos un escaneo exhaustivo del plano $\tilde{A}>0, J_{2} / J_{1}>0$ para $N=24$ sitios, manteniendo $\tilde{B}=1.5 \tilde{A}$. El diagrama de fases encontrado es mostrado en la figura 4.20. Escaneos representativos en $J_{2} / J_{1}=0.3,0.6,0.9$ son mostrados en la figura 4.21, mostrando $\delta_{1}, \delta_{2}, \delta_{3}$ y $M_{S}$ como función de $\tilde{A}$. Notemos que la amplitud de la deformación decrece para valores pequeños de $\tilde{A}$ (un límite que corresponde a grandes valores de $K$ ); debido a los efectos de tamaño finito [139] el sistema no es inestable frente a las deformaciones para valores muy pequeños de $\tilde{A}$. El escaneo para $J_{2} / J_{1}=0.3$ muestra las fases $D, Q P$ para todo $\tilde{A}$. En el caso $J_{2} / J_{1}=0.6$ se puede observar una transición en algún valor de $\tilde{A}$ (que en general depende de $J_{2} / J_{1}$ ), con un salto finito ambos en las deformaciones como en el parámetro de orden magnético, desde las fase $T, C P$ a la fase $D, Q P$. Lo mismo sucede en el caso $J_{2} / J_{1}=0.9$, en el que una transición desde la fase $D, C P$ a la fase $T, Q P$. La

\footnotetext{
${ }^{2}$ Esta parte del trabajo fue realizada con la colaboración del Dr. Marcelo D. Grynberg.
} 


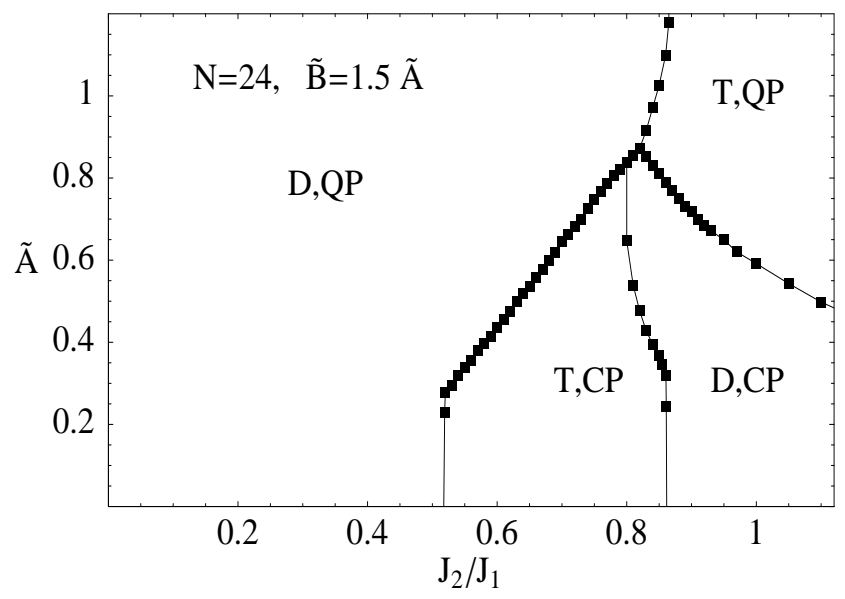

Figura 4.20: Diagrama de fases magneto-elástico obtenido mediante diagonalización exacta de $N=24$ sitios. La relación entre los acoplamientos espín-fonón es fijada a $\tilde{B} / \tilde{A}=1.5$.

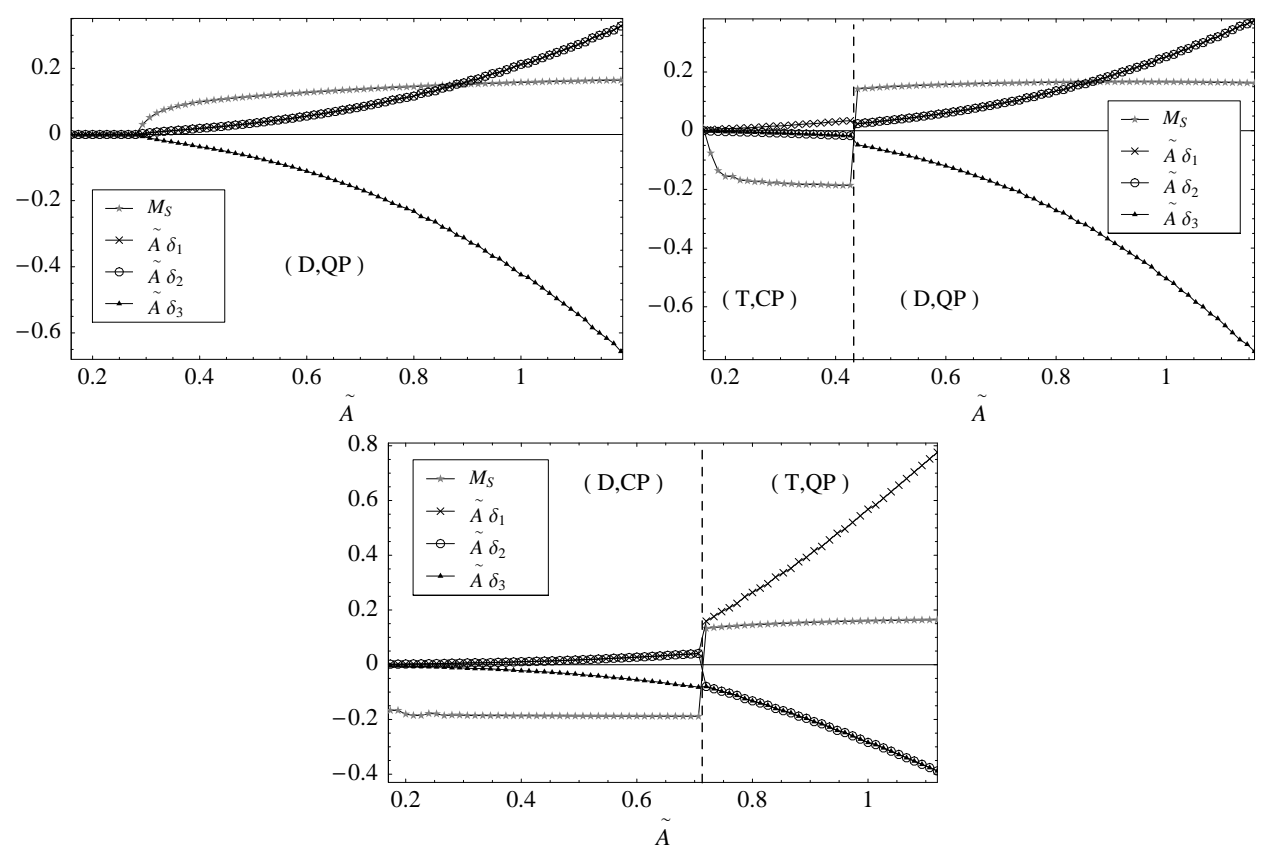

Figura 4.21: Distorsiones de la red $\delta_{1,2,3}$ y parámetro de orden $M_{S}$ como función de $\tilde{A}$ para $\tilde{B} / \tilde{A}=1.5$ y $J_{2} / J_{1}=0.3$ (panel superior), 0.6 (panel medio), 0.9 (panel inferior). Cada panel corresponde a un recorrido vertical en la figura 4.20. Los valores pequeños de $\tilde{A}$ no son accesibles debido a efectos de tamaño finito. 
región $0.8<J_{2} / J_{1}<0.9$ muestra que la línea crítica para la transición entre las fases $T$ y $D$ dependen levemente de $J_{2} / J_{1}$; comparando con la figura 4.18 indica que los efectos de renormalización en los coeficientes $f_{1}, f_{2}$ no son suficientemente fuertes como para impedir nuestro análisis cualitativo.

Adicionalmente hemos analizado diferentes acoplamientos espín-fonón, confirmando las predicciones de bosonización que disminuyendo la relación de $\tilde{B} / \tilde{A}$ produce un incremento de la región caracterizada por la fase de plateau clásico y la deformación trimerizada (c.f. figura 4.18), con valores más altos tanto de $\alpha_{c 1}$ como de $\tilde{A}_{c}$.

\subsubsection{Conclusiones}

En la presente sección investigamos la posibilidad de que el acoplamiento del sector de espín en la cadena antiferromagnética $J_{1}-J_{2}$ de $S=1 / 2$ a magnetización $M=1 / 3$, con fonones en el límite adiabático produzca modulaciones que seleccionen las fases magnéticas estudiadas en la sección 4.2.1: plateau clásico y plateau cuántico. Realizamos una análisis semiclásico de la teoría efectiva obtenida por medio de bosonización abeliana, apoyado por diagonalización exacta de sistemas pequeños de hasta 24 espines. Encontramos varias fases del tipo "espín-Peierls" que describen el estado fundamental del sistema, dependiendo de los parámetros microscópicos $J_{2} / J_{1}$ y de los acoplamientos espín-fonón. En cada una de estas fases una deformación elástica no trivial es favorecida, agrupando bloques de dos o tres espines, mientras que el sector magnético adopta estados de plateau clásico o cuántico.

Realizamos un análisis detallado de un caso particular, escogido como representativos de materiales donde la relación de acoplamientos espín-fonón a segundos y primeros vecinos [138] es grande, mostrando los siguientes resultados:

(i) una fase magnética $\uparrow \uparrow \downarrow$ con una distorsión de la red trimerizada cuando la frustración es suficientemente grande y el acoplamiento espín-fonón es pequeño.

(ii) una fase magnética $\uparrow \uparrow \downarrow$ con distorsión de la red dimerizada para mayor frustración y acoplamiento espín-fonón pequeño.

(iii) una fase magnética tipo plateau cuántico con una distorsión dimerizada para valores grandes del acoplamiento espín-fonón y débil frustración.

(iv) una fase magnética tipo plateau cuántico con una distorsión trimerizada para valores grandes del acoplamiento espín-fonón y alta frustración. 

Parte II

Sistemas Magnéticos en 2D 



\section{Capítulo 5}

\section{Propiedades Magnéticas del Modelo de Heisenberg en 2D}

En los capitúlos anteriores estudiamos las propiedades del estado fundamental de sistemas unidimensionales modelados por hamiltonianos de Hubbard y Heisenberg a temperatura cero. La ventaja de estudiar estos sistemas se basa en que estos pueden ser analizados mediante una gran variedad de técnicas análiticas y numéricas, que permiten explorar con mucha profundidad sus propiedades. En contraste, el caso de sistemas bidimensionales es mucho más delicado debido a que, por un lado las técnicas análiticas poseen distintos rangos de aplicabilidad y éstas dependen fuertemente del modelo estudiado; y por otro las técnicas numéricas son limitadas a estudiar tamaños muy pequeños dando solo indicios del comportamiento de un sistema macroscópico. Estas limitaciones tienen como consecuencia un desconocimiento general de los sistemas bidimensionales y que muchas de sus propiedades estén bajo discusión acualmente .

En este capítulo revisaremos las propiedades magnéticas del modelo de Heisenberg en dos redes bidimensionales. En la primera parte estudiamos el modelo de Heisenberg en la red de Kagomé utilizando la teoría de campo medio de bosones de Schwinger. Además del acoplamiento estandar a primeros vecinos, consideramos un acoplamiento a terceros vecinos enfocándonos en la influencia de éste sobre la propiedades magnéticas del estado fundamental. Parte de la motivación surge por el descubrimiento de nuevos materiales con la estructura de la red de Kagomé con estos tipos de acoplamientos.

En la segunda parte, presentamos el estudio realizado ${ }^{1}$ del modelo de Heisenberg en la red hexagonal con interacciones antiferromagnéticas a primeros, segundos y terceros vecinos, enfocándonos principalmente en la búsqueda de fases magnéticamente desordenadas.

\footnotetext{
${ }^{1}$ Este trabajo se realizó en colaboración con el Dr. Daniel Cabra y el Lic. Carlos Lamas, y puede ser encontrado en la tesis doctoral de éste último.
} 


\subsection{Modelo de Heisenberg en la red de Kagomé.}

\subsubsection{Introducción}

Los sistemas cuánticos antiferromagnéticos geométricamente frustrados en dos dimensiones (2D) son considerados como posibles candidatos para desarrollar un estado fundamental desordenado magnéticamente a temperatura cero. El primer candidato sugerido como líquido de espín fue la red triangular debido a que su geometría es considerada menos conducente a desarrollar orden magnético en comparación por ejemplo con la red cuadrada antiferromagnética. Sin embargo numerosos estudios han revelado el modelo de Heisenberg con interacciones solo a primeros vecinos exhibe un orden de largo alcance donde los valores de espectación de los operadores de espín muestran una estructura conocida como orden de $120^{\circ}$ $[140,141,142,143,144]$. Posteriormente se han llevado a cabo estudios numéricos incluyendo en el modelo anisotropía o introduciendo interacciones adicionales como ring exchange $[145,146,147,148]$.

En los últimos años la red de Kagomé antiferromagnética se ha vuelto el centro de atención en esta área, tanto desde punto de vista experimental como teórico. La actividad en la red de Kagomé antiferromagnética inicialmente surgió como modelo para describir dos sistemas experimentales: el compuesto aislador $\mathrm{SrCr}_{8-x} \mathrm{Ga}_{4+x} \mathrm{O}_{19}$, donde los iones de $\mathrm{Cr}$ con espín $S=3 / 2$ forman arreglos bidimensionales con la estructura de la red de Kagomé separados por capas de redes triangulares [149]; y un segundo sistema formado por ${ }^{3} \mathrm{He}$ absorbido en grafito [150] con espín $S=1 / 2$. Desde el punto de vista teórico el modelo cuántico es particularmente interesante debido a que su contraparte clásica, es decir el hamiltoniano de Heisenberg clásico (ver sección 1.3), presenta un estado fundamental macroscópicamente degenerado (ver sección 1.3.4) y un bajo número de coordinación $(z=4)$.

Recientemente Janson et al $[151,152]$ realizaron cálculos de DFT (Density functional theory) en dos nuevos materiales isoestructurales con la red de Kagomé de espín $S=1 / 2$ - kapellasite $\mathrm{Cu}_{3} \mathrm{Zn}(\mathrm{OH})_{6} \mathrm{Cl}_{2}$ y haydeeite $\mathrm{Cu}_{3} \mathrm{Mg}(\mathrm{OH})_{6} \mathrm{Cl}_{2}$ - mostrando que además del modelo basado en acoplamientos a primeros vecinos $\left(J_{1}\right)$, estos materiales presentan un acoplamiento adicional relevante $J_{d}>0$ a lo largo de las diagonales de los hexagonos de la red (figura 5.2) encontrando que la relación de acoplamientos son $J_{d} / J_{1} \simeq 0.36$ para kapellasite y $J_{d} / J_{1} \simeq 1$ para haydeeite. Con esto, el hamiltoniano de Heisenberg que puede describir los compuestos antes mencioandos está dado por

$$
H=J_{1} \sum_{\langle i, j\rangle} \mathbf{S}_{\mathbf{r}_{i}} \cdot \mathbf{S}_{\mathbf{r}_{j}}+J_{d} \sum_{\langle\langle i, j\rangle\rangle} \mathbf{S}_{\mathbf{r}_{i}} \cdot \mathbf{S}_{\mathbf{r}_{j}},
$$

donde $i$ y $j$ son los índices de sitios y $\mathbf{S}_{\mathbf{r}}$ son los operadores de espín. La notación $\langle i, j\rangle$ $(\langle\langle i, j\rangle\rangle)$ indica que la suma se hace entre sitios a primeros (terceros) vecinos.

Como hemos mencionado anteriormente el estado fundamental del modelo de Heisenberg clásico $^{2}$ en la red de kagomé pura $\left(J_{d}=0\right)$ posee una degeneración macroscópica (ver sección 1.3). Es de esperar que el acoplamiento adicional $J_{d}$ reduzca ésta degeneración drásticamente

\footnotetext{
${ }^{2}$ Recordemos que cada vez que hacemos mención del modelo clásico de Heisenberg, nos estamos refiriendo
} 


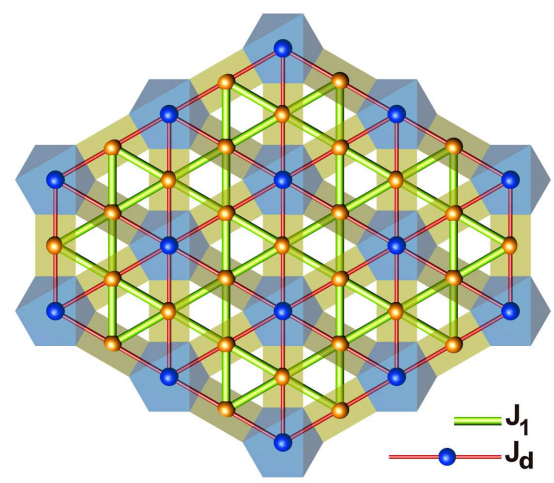

Figura 5.1: Planos de Kagomé en la estructura de Kapellasite (haydeeeite). Las plaquetas de $\mathrm{CuO}_{4}$ son mostradas en amarillo, los octaedros $\mathrm{ZnO}_{6}\left(\mathrm{MgO}_{6}\right)$ son mostrados en azul. Figura extraída de J. Phys.: Conf. Ser. 145012008 [151].

seleccionando alguna configuración determinada. En particular, recientes trabajos sobre el modelo clásico sugieren que tal configuración corresponde a un orden no planar de los espines [151] como se muestra en la figura 5.2.

En cuanto al modelo de Heisenberg cuántico, Janson et al [151] calcularon las correlaciones

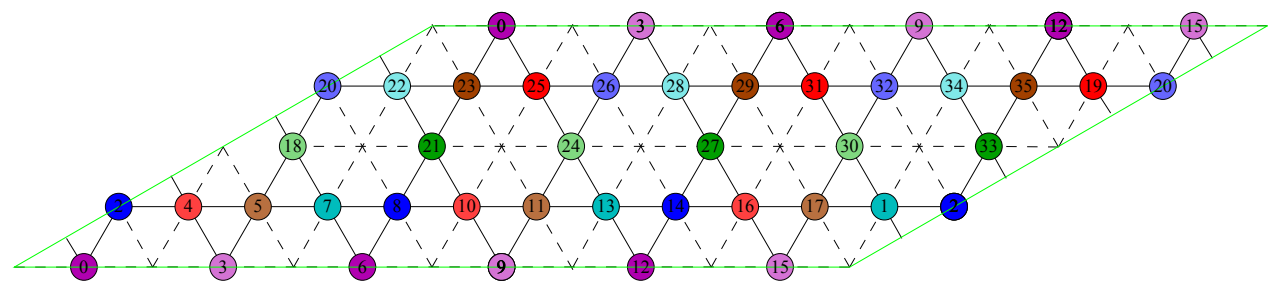

Figura 5.2: Red de Kagomé de $N=36$ sitios. Las líneas sólidas representan los acoplamientos $J_{1}$ y las lineas a trazos $J_{d}$. Los círculos coloreados indican las orientaciones de los espines del estado fundamental del modelo clásico para $J_{d}>0$. A lo largo de las cadenas formadas por los bonds diagonales hay un alineamiento antiparalelo. Dentro de cada arreglo triangular formado por los acoplamientos $J_{1}$ los espines forman un ángulo de $120^{\circ}$. Figura extraída de J. Phys.: Conf. Ser. 145012008 [151].

espín-espín mediante diagonalización exacta de redes de hasta 36 sitios con el objetivo de identificar el tipo de orden magnético del estado fundamental. En la figura 5.3 se observa que para $J_{d} \simeq 0$ todas las correlaciones espín-espín excepto $\left\langle\mathbf{S}_{0} \mathbf{S}_{1}\right\rangle$ son despreciables. Para $0.55 \lesssim J_{d} / J_{1}$ las correlaciones $\left\langle\mathbf{S}_{0} \mathbf{S}_{3}\right\rangle,\left\langle\mathbf{S}_{0} \mathbf{S}_{6}\right\rangle$ y $\left\langle\mathbf{S}_{0} \mathbf{S}_{9}\right\rangle$ son las más importantes revelando

a la situación en la cual los espines son tratados como vectores de magnitud $S$. En muchas situaciones el estudio de éste modelo es de gran ayuda a la hora de analizar los estados magneticamente ordenados del modelo cuántico. 
un orden tipo Néel a lo largo de las lineas diagonales.

Es nuestro interés analizar la influencia de $J_{d}$ en el límite termodinámico, para lo cual en

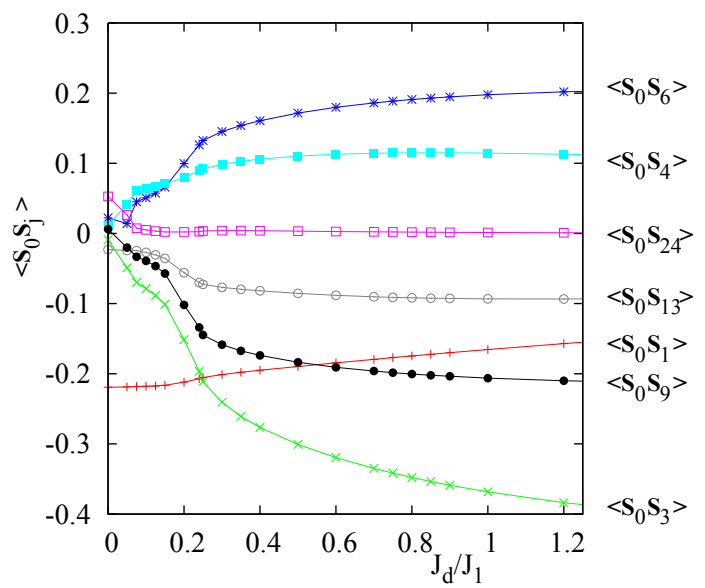

Figura 5.3: Correlación espín-espín $\left\langle\mathbf{S}_{0} \mathbf{S}_{j}\right\rangle$ en función de $J_{d} / J$ para la red finita de $N=36$ sitios mostrada en la figura 5.2. Los índices de sitio $j=1,3,4,6,9,13,24$ corresponden a aquellos de la figura 5.2. Figura extraída de J. Phys.: Conf. Ser. 145012008 [151].

la sección siguiente vamos a estudiar el hamiltoniano (5.1) utilizando el método de campo medio de bosones de Schwinger desarrollado en la sección 2.2.2.

\subsubsection{Métodos de bosones de Schwinger}

En esta sección presentamos el estudio realizado del hamiltoniano (5.1) que va en paralelo con un trabajo previo [153] utilizando el método de bosones de Schwinger introducido en la sección 2.2.2. Utilizando el mapeo de los operadores de espín en términos de los operadores bosónicos dado por las ecuaciones (2.82) y (2.83) el hamiltoniano puede ser expresado como

$$
\begin{aligned}
\mathcal{H}= & \sum_{\langle i, j ; a, b\rangle} J_{1}\left(: \mathbf{B}_{\mathbf{r}_{i}, \mathbf{r}_{j}}^{\dagger, a, b} \cdot \mathbf{B}_{\mathbf{r}_{i}, \mathbf{r}_{j}}^{a, b}:-\mathbf{A}_{\mathbf{r}_{i}, \mathbf{r}_{j}}^{\dagger, a, b} \cdot \mathbf{A}_{\mathbf{r}_{i}, \mathbf{r}_{j}}^{a, b}\right)+\sum_{\langle\langle i, j ; a, b\rangle\rangle} J_{d}\left(: \mathbf{B}_{\mathbf{r}_{i}, \mathbf{r}_{j}}^{\dagger, a, b} \cdot \mathbf{B}_{\mathbf{r}_{i}, \mathbf{r}_{j}}^{a, b}:-\mathbf{A}_{\mathbf{r}_{i}, \mathbf{r}_{j}}^{\dagger, a, b} \cdot \mathbf{A}_{\mathbf{r}_{i}, \mathbf{r}_{j}}^{a, b}\right) \\
& +\sum_{i ; a} \lambda_{\mathbf{r}_{i}}^{a}\left(b_{\mathbf{r}_{i}, \uparrow}^{\dagger, a} b_{\mathbf{r}_{i}, \uparrow}^{a}+b_{\mathbf{r}_{i}, \downarrow}^{\dagger, a} b_{\mathbf{r}_{i}, \downarrow}^{a}-2 S\right),
\end{aligned}
$$

donde los índices $i, j$ recorren la red de Bravais periódica (una red triangular); $a$ y $b$ son índices internos de subred $u, v$ o $w$ como se indican en la figura (5.4), y la notación \langle\rangle y $\langle\langle\rangle\rangle$ significan todos los posibles pares de sitios a primeros y terceros vecinos respectivamente. $N_{\Lambda}$ es el número de celdas primitivas (así, el número total de sitios en la red es $3 N_{\Lambda}$ ) y $\lambda_{\mathbf{r}_{i}}$ son multiplicadores de Lagrange introducidos para realizar la restricción (en valor medio) del número de bosones en cada sitio, y : $\mathbf{O}$ : indicando orden normal de los operadores. Debido a la invarianza traslacional vamos a fijar $\lambda_{\mathbf{r}_{i}}^{a}=\lambda$. Finalmente los operadores de bonds $\mathbf{A}_{\mathbf{r}_{i} ; \mathbf{r}_{j}}$ 

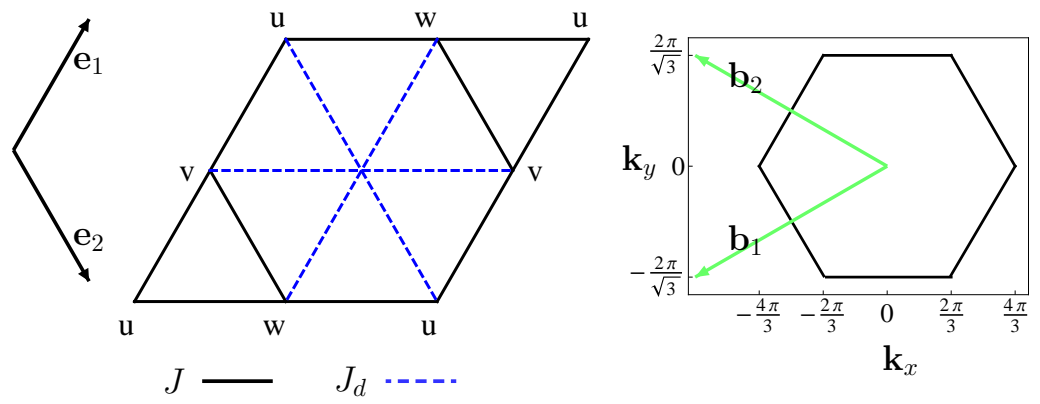

Figura 5.4: Celda primitiva y primera zona de Brillouin de la red de Kagomé. Los vectores primitivos de la red directa y reciproca son $\left(\mathbf{e}_{1}=(1 / 2, \sqrt{3} / 2), \mathbf{e}_{2}=(1 / 2, \sqrt{3} / 2)\right)$ y $\left(\mathbf{b}_{1}=-2 \pi(1,1 / \sqrt{3}), \mathbf{b}_{2}=-2 \pi(1,-1 / \sqrt{3})\right)$, respectivamente.

y $\mathbf{B}_{\mathbf{r}_{i} ; \mathbf{r}_{j}}$ están dados por

$$
\begin{aligned}
\mathbf{A}_{\mathbf{r}_{i} ; \mathbf{r}_{j}}^{a, b} & \equiv \frac{1}{2}\left(b_{\mathbf{r}_{i}, \uparrow}^{a} b_{\mathbf{r}_{j}, \downarrow}^{b}-b_{\mathbf{r}_{i}, \downarrow}^{a} b_{\mathbf{r}_{j}, \uparrow}^{b}\right) \\
\mathbf{B}_{\mathbf{r}_{i} ; \mathbf{r}_{j}}^{a, b} & \equiv \frac{1}{2}\left(b_{\mathbf{r}_{i}, \uparrow}^{\dagger, a} b_{\mathbf{r}_{j}, \uparrow}^{b}+b_{\mathbf{r}_{i}, \downarrow}^{\dagger, a} b_{\mathbf{r}_{j}, \downarrow}^{b}\right),
\end{aligned}
$$

Para poder resolver este problema, ahora cuártico en bosones, se procede a un desacople en campo medio de los operadores de bonds introduciendo los parámetros $\left\langle\mathbf{A}_{\mathbf{r}_{i}, \mathbf{r}_{j}}^{a, b}\right\rangle=A_{\alpha} \mathrm{y}$ $\left\langle\mathbf{B}_{\mathbf{r}_{i}, \mathbf{r}_{j}}^{a, b}\right\rangle=B_{\alpha}$ ( $\alpha=1,2$ primeros y terceros vecinos) desarrollado en la sección 2.2.2. Luego de transformar Fourier los operadores bosónicos, para cada modo $k$ es conveniente introducir el vector de operadores $\Psi(\mathbf{k})=\left(b_{\mathbf{k}, \uparrow}^{u}, b_{\mathbf{k}, \uparrow}^{v}, b_{\mathbf{k}, \uparrow}^{w}, b_{-\mathbf{k}, \downarrow}^{\dagger, u}, b_{-\mathbf{k}, \downarrow}^{\dagger, v}, b_{-\mathbf{k}, \downarrow}^{\dagger, c}\right)$. Con esto podemos reescribir el hamiltoniano en la aproximación de campo medio de una forma más compacta

$$
\mathcal{H}_{M F}=\epsilon_{0}+\sum_{\mathbf{k} \in B Z} \Psi^{\dagger}(\mathbf{k}) \cdot M(\mathbf{k}) \cdot \Psi(\mathbf{k})
$$

donde

$$
\frac{\epsilon_{0}}{N_{\Lambda}}=\left[6 J_{1}\left(B_{1}^{2}-A_{1}^{2}\right)+3 J_{d}\left(B_{2}^{2}-A_{2}^{2}\right)\right]-3 \lambda(2 S+1)
$$

y las matrices $M(\mathbf{k}), P(\mathbf{k})$ y $R(\mathbf{k})$ están dados por

$$
\begin{gathered}
M(\mathbf{k})=\left[\begin{array}{cc}
\lambda \mathrm{I}_{3 \times 3}+R(\mathbf{k}) & -P(\mathbf{k}) \\
-P^{\dagger}(\mathbf{k}) & \lambda \mathrm{I}_{3 \times 3}+R(\mathbf{k})
\end{array}\right] \\
P(\mathbf{k})=\left[\begin{array}{ccc}
J_{2} A_{2} D_{2}(\mathbf{k}) & J_{1} A_{1} \Gamma_{1}(-\mathbf{k}) & -J_{1} A_{1} \Gamma_{3}(\mathbf{k}) \\
-J_{1} A_{1} \Gamma_{1}(\mathbf{k}) & J_{2} A_{2} D_{3}(\mathbf{k}) & J_{1} A_{1} \Gamma_{2}(-\mathbf{k}) \\
J_{1} A_{1} \Gamma_{3}(-\mathbf{k}) & -J_{1} A_{1} \Gamma_{2}(\mathbf{k}) & J_{2} A_{2} D_{1}(\mathbf{k})
\end{array}\right],
\end{gathered}
$$




$$
\begin{aligned}
& \operatorname{con} \Gamma_{j}(\mathbf{k})=e^{i \mathbf{k} \cdot \vec{e}_{j} / 2}+\sigma e^{-i \mathbf{k} \cdot \vec{e}_{j} / 2} \mathrm{y} D_{j}(\mathbf{k})=-2 i \sin \left(\mathbf{k} \cdot \vec{e}_{j}\right), \mathrm{y} \\
& R(\mathbf{k})=2\left[\begin{array}{ccc}
J_{2} B_{2} \cos \left(\mathbf{k} \cdot \vec{e}_{2}\right) & J_{1} B_{1} \cos \left(\mathbf{k} \cdot \vec{e}_{1} / 2\right) & J_{1} B_{1} \cos \left(\mathbf{k} \cdot \vec{e}_{3} / 2\right) \\
J_{1} B_{1} \cos \left(\mathbf{k} \cdot \vec{e}_{1} / 2\right) & J_{2} B_{2} \cos \left(\mathbf{k} \cdot \vec{e}_{3}\right) & J_{1} B_{1} \cos \left(\mathbf{k} \cdot \vec{e}_{2} / 2\right) \\
J_{1} B_{1} \cos \left(\mathbf{k} \cdot \vec{e}_{3} / 2\right) & J_{1} B_{1} \cos \left(\mathbf{k} \cdot \vec{e}_{2} / 2\right) & J_{2} B_{2} \cos \left(\mathbf{k} \cdot \vec{e}_{1}\right)
\end{array}\right],
\end{aligned}
$$

Para determinar los autovalores del hamiltoniano (5.5), procedemos a diagonalizar mediante una transformación de Bogoliubov generalizada o también llamada paraunitaria mostrada en el apéndice $\mathrm{B}$, tal que $\Psi(\mathbf{k})=T \tilde{\Psi}(\mathbf{k})$ introduciendo un nuevo conjunto de operadores $\tilde{b}_{\mathbf{k}, \sigma}^{a}$ como se detalla en el apéndice B.

Finalmente el hamiltoniano de campo medio (5.5) queda

$$
\begin{aligned}
\mathcal{H}_{M F}= & \epsilon_{0}+2 \sum_{\mathbf{k}, \sigma=\uparrow \downarrow} w_{1}(\mathbf{k})\left(\tilde{b}_{\mathbf{k}, \sigma}^{\dagger, u} \tilde{b}_{\mathbf{k}, \sigma}^{u}+\frac{1}{2}\right)+w_{2}(\mathbf{k})\left(\tilde{b}_{\mathbf{k}, \sigma}^{\dagger, v} \tilde{b}_{\mathbf{k}, \sigma}^{v}+\frac{1}{2}\right) \\
& +w_{3}(\mathbf{k})\left(\tilde{b}_{\mathbf{k}, \sigma}^{\dagger, w} \tilde{b}_{\mathbf{k}, \sigma}^{w}+\frac{1}{2}\right)
\end{aligned}
$$

y la energía del estado fundamental se obtiene a partir del valor de espectación del hamiltoniano $\mathcal{H}_{M F}$ en el vacío del espacio de Fock de los nuevos bosones $\tilde{b}$,

$$
\begin{aligned}
E_{G S} & =\left\langle\mathcal{H}_{M F}\right\rangle \\
& =\epsilon_{0}+\sum_{\mathbf{k} \in B Z}\left(w_{1}(\mathbf{k})+w_{2}(\mathbf{k})+w_{3}(\mathbf{k})\right)
\end{aligned}
$$

donde $w(\mathbf{k})$ son bandas de energías que dependen de los parámetros de campo medio $A_{\alpha}$, $B_{\alpha}$ y del multiplicador de Lagrange $\lambda$ a ser determinados autoconsistentemente a partir de los valores medios de los operadores $\mathbf{A}$ y $\mathbf{B}$

$$
\begin{aligned}
& A_{1}=\frac{1}{6 N_{\Lambda}} \sum_{\left\langle\mathbf{r}_{i}, \mathbf{r}_{j}\right\rangle}\left\langle b_{\mathbf{r}_{i}, \uparrow}^{\dagger, a} b_{\mathbf{r}_{j}, \downarrow}^{\dagger, b}-b_{\mathbf{r}_{i}, \downarrow}^{\dagger, a} b_{\mathbf{r}_{j}, \uparrow}^{\dagger, b}\right\rangle \\
& A_{2}=\frac{1}{3 N_{\Lambda}} \sum_{\left\langle\left\langle\mathbf{r}_{i}, \mathbf{r}_{j}\right\rangle\right\rangle}\left\langle b_{\mathbf{r}_{i}, \uparrow}^{\dagger, a} b_{\mathbf{r}_{j}, \downarrow}^{\dagger, b}-b_{\mathbf{r}_{i}, \downarrow}^{\dagger, a} b_{\mathbf{r}_{j}, \uparrow}^{\dagger, b}\right\rangle \\
& B_{1}=\frac{1}{6 N_{\Lambda}} \sum_{\left\langle\mathbf{r}_{i}, \mathbf{r}_{j}\right\rangle}\left\langle b_{\mathbf{r}_{i}, \uparrow}^{\dagger, a} b_{\mathbf{r}_{j}, \downarrow}^{b}+b_{\mathbf{r}_{i, \downarrow}}^{\dagger, a} b_{\mathbf{r}_{j}, \uparrow}^{b}\right\rangle \\
& B_{2}=\frac{1}{3 N_{\Lambda}} \sum_{\left\langle\left\langle\mathbf{r}_{i}, \mathbf{r}_{j}\right\rangle\right\rangle}\left\langle b_{\mathbf{r}_{i}, \uparrow}^{\dagger, a} b_{\mathbf{r}_{j}, \downarrow}^{b}+b_{\mathbf{r}_{i}, \downarrow}^{\dagger, a} b_{\mathbf{r}_{j}, \uparrow}^{b}\right\rangle
\end{aligned}
$$

junto con la ecuación que fija el número de bosones en cada sitio

$$
\frac{1}{N_{\Lambda}} \sum_{\mathbf{r}_{i}, \sigma}\left\langle b_{\mathbf{r}_{i}, \sigma}^{\dagger, a} b_{\mathbf{r}_{i}, \sigma}^{a}\right\rangle=2 S .
$$


Obtener las soluciones numéricas de las ecuaciones (5.12) y (5.13) involucra resolver cuatro ecuaciones acopladas para los parámetros $A^{\prime} s$ y $B^{\prime} s$, más la ecuación de ligadura para determinar el valor de $\lambda$. El procedimiento autoconsistente consiste en proponer valores iniciales para los parámetros $A_{\alpha}^{0}$ y $B_{\alpha}^{0}$, y determinar la cota mínima $\lambda_{\text {min }}^{(0)}$ del multiplicador de Lagrange para el que todas las matrices $M(\mathbf{k})$ en ecuación (5.5) son definidas positivas. Resolvemos la ecuación (5.13) en el intervalo $\left[\lambda_{\text {min }}^{(0)}, \infty\right)$ para determinar el valor crítico $\lambda_{c}^{(0)}$. Con los valores de los parámetros de campo medio y el multiplicador de Lagrange calculamos la energía y los nuevos parámetros $A_{\alpha}^{(1)}$ y $B_{\alpha}^{(1)}$. Con estos nuevos valores, determinamos el nuevo $\lambda_{\text {min }}^{(1)}$ y determinamos el $\lambda_{c}^{(1)}$ y los nuevos parámetros $A_{\alpha}^{(2)}$ y $B_{\alpha}^{(2)}$. El procedimiento es repetido hasta que los parámetros $A_{\alpha}^{(n)}, B_{\alpha}^{(n)}$ y $\lambda_{c}^{(n)}$ convergen, así como la energía converge al valor mínimo. La necesidad de resolver numéricamente limita estudiar sistemas finitos pero suficientemente grandes como parea extrapolar los resultados al límite termodinámico 3 .

\subsubsection{Resultados}

La evaluación numérica de las ecuaciones (5.29) y (5.30) muestran que para para $0 \leq$ $J_{d} / J_{1} \leq \infty$ el modelo (5.1) no posee gap en su espectro de excitaciones para valores de espín $S \geq 1 / 2$ indicando la presencia de orden de largo alcance en el estado fundamental. Por lo tanto $J_{d}$ estabiliza orden magnético descartando la posibilidad de una fase desordenada tipo líquido de espines.

Como ejemplo en la figura 5.5 se muestra la banda de energía más baja de las cuasipartículas en el plano $k_{x}-k_{y}$ y un gráfico de densidad para $J_{d} / J_{1}=0.3$ y espín $S=1 / 2$. Se observa que los ceros de la relación de dispersión $w\left(\mathbf{k}^{*}\right)=0$ se ubican en los puntos $\mathbf{k}^{*}=\left(\frac{4 \pi}{3}, 0\right)$ y $\mathbf{k}^{*}=\left(\frac{2 \pi}{3}, \pm \frac{2 \pi}{\sqrt{3}}\right)$. A partir del cálculo de los valores de espectación de los operadores de
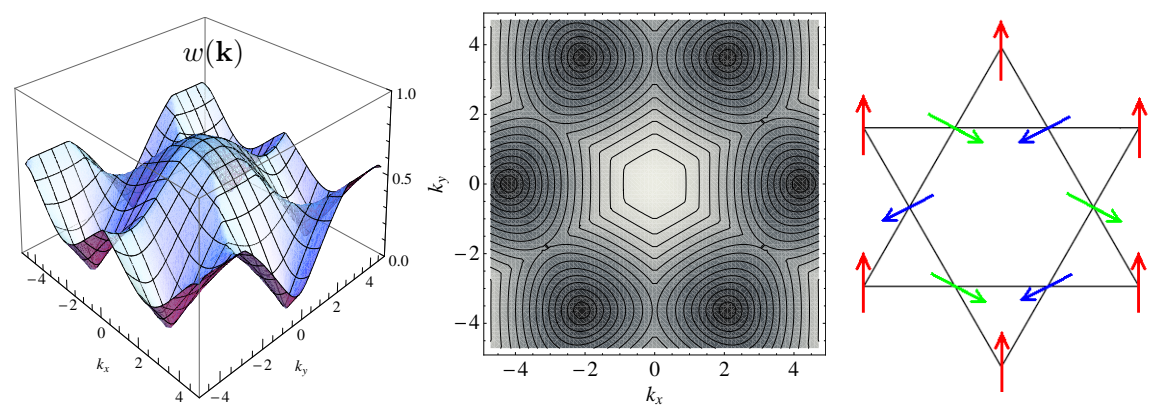

Figura 5.5: (Izquierda y centro) Espectro de cuasi-partículas y gráfico de densidad para $J_{d} / J_{1}=0.3$. El espectro posse puntos sin gap, es decir $w\left(\mathbf{k}^{*}\right)=0$ en $\mathbf{k}^{*}=\left(\frac{4 \pi}{3}, 0\right) \mathrm{y}$ $\mathbf{k}^{*}=\left(\frac{2 \pi}{3}, \pm \frac{2 \pi}{\sqrt{3}}\right)$ que corresponden al orden magnético $\sqrt{3} \times \sqrt{3}$. (Derecha) Orden $\sqrt{3} \times \sqrt{3}$ establecido para $0 \leq J_{d} / J_{1} \leq \infty$.

espín en términos de los operadores bosonicos originales se puede inferir el tipo de orden que presenta el estado fundamental. Los resultados muestran que la inclusión del acoplamiento $J_{d}$

\footnotetext{
${ }^{3}$ Generalmente, los resultados para sistemas más grandes que 3600 sitios no muestran fuerte dependencia con el tamaño.
} 
estabiliza el estado ordenado conocido en la literatura como $\sqrt{3} \times \sqrt{3}$ [154] esquematizado en la figura (5.5 panel izquierdo) pudiendo ser contrastado experimentalmente en los compuestos anteriormente citados kapellasite $\mathrm{Cu}_{3} \mathrm{Zn}(\mathrm{OH})_{6} \mathrm{Cl}_{2}$ y haydeeite $\mathrm{Cu}_{3} \mathrm{Mg}(\mathrm{OH})_{6} \mathrm{Cl}_{2}$.

\subsection{Modelo de Heisenberg en la red hexagonal.}

En la sección anterior estudiamos el estado fundamental de la red de Kagomé con la inclusión de una acoplamiento adicional $J_{d}$ el cual estabiliza un orden magnético. Originalmente la red de Kagomé (con $J_{d}=0$ ) atrajo mucha atención debido a que la red en sí es frustrada y además el modelo clásico posee una degeneración macroscópica en el estado fundamental. Estos ingredientes se cree que pueden ser mecanismos para la formación de una fase desordenada o líquido de espín del modelo cuántico (ver sección 1.3.3). Otro candidato para testear estas ideas es el modelo de Heisenberg antiferromagnético en la red hexagonal o panal de abejas debido al bajo número de coordinación que posee $(z=3$ el menor posible en redes bidimensionales) y por lo tanto se espera que las fluctuaciones cúanticas puedan ser suficientemente grandes como para destruir el orden de largo alcance. Numerosos estudios muestran que el estado fundamental del modelo cuántico en la red hexagonal es ordenado con una estructura similar al estado de Néel mostrado en la figura (5.9). Cabe preguntarse si la inclusión de acoplamientos a siguientes vecinos que introduzcan frustración pueden competir con el orden.

El estudio de los magnetos cuánticos en la red hexagonal tiene además varias motivaciones experimentales: por un lado, recientes experimentos de ESR (Electrón Spin Resonance) [155] en campos magnéticos elevados en el compuesto $\mathrm{Bi}_{2} \mathrm{Mn}_{4} \mathrm{O}_{12}\left(\mathrm{NO}_{3}\right)$, que es descrito por un modelo de Heisenberg en la red hexagonal con espín $S=3 / 2$, han conjeturado que la frustración magnética puede jugar un rol importante para remover el orden de largo alcance [156]. Por otro lado, orden de corto alcance debajo de $50 \mathrm{~K}$ ha sido observado en otro material con estructura hexagonal $\mathrm{InCu}_{2 / 3} \mathrm{~V}_{1 / 3} \mathrm{O}_{3}$ (figura 5.7(a)) donde los iones no magnéticos de Vanadio pueden conducir a frustración magnética [157]. Finalmente mencionamos que el material $\mathrm{Na}_{3} \mathrm{Cu}_{2} \mathrm{SbO}_{6}$ [158] que posee una gap de espín posee una estructura que aún se encuentra bajo debate pero podría ser descrita por una red hexagonal anisotrópica como se muestra en la figura 5.7(b)).

El modelo de Heisenberg en la red hexagonal con acoplamientos antiferromagnéticos a primeros $\left(J_{1}\right)$, segundos $\left(J_{2}\right)$ y terceros $J_{3}$ vecinos [159] está dado por,

$$
H=J_{1} \sum_{\langle i, j\rangle} \mathbf{S}_{\vec{r}_{i}} \cdot \mathbf{S}_{\vec{r}_{j}}+J_{2} \sum_{\langle\langle i, j\rangle\rangle} \mathbf{S}_{\vec{r}_{i}} \cdot \mathbf{S}_{\vec{r}_{j}}+J_{3} \sum_{\langle\langle\langle i, j\rangle\rangle\rangle} \mathbf{S}_{\vec{r}_{i}} \cdot \mathbf{S}_{\vec{r}_{j}}
$$

donde $\mathbf{S}_{\vec{r}_{i}}$ es el operador de espín en el sitio $i$-ésimo y \langle\rangle$,\langle\langle\rangle\rangle$ y $\langle\langle\langle\rangle\rangle\rangle$ indican que las sumas involucran sitios $i, j$ a primeros, segundos y terceros vecinos respectivamente (ver figura $(5.7))$.

Más allá que la frustración se debe al acoplamiento $J_{2}$, como veremos la inclusión de $J_{3}$ tiene una consecuencia importante. El límite clásico $(S \rightarrow \infty)$ del hamiltoniano (5.14) ha sido 


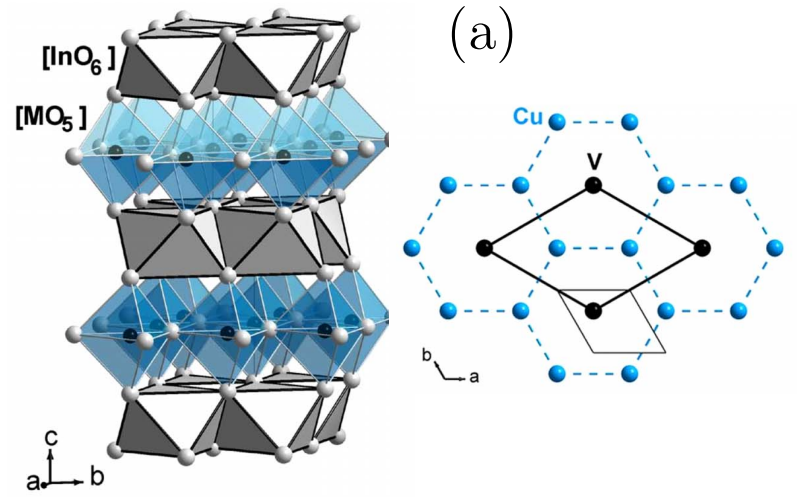

(b)

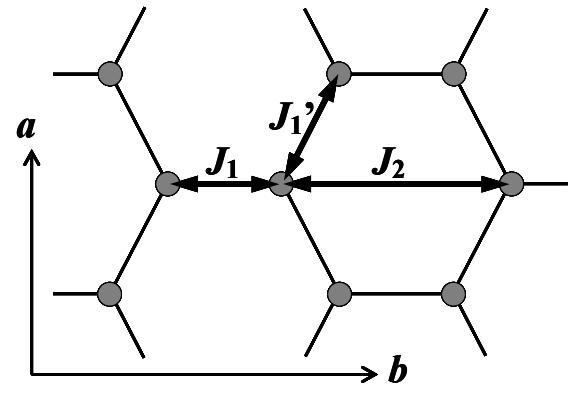

Figura 5.6: (a) Vista perspectiva de la estructura cristalina del compuesto $\mathrm{InCu}_{2 / 3} \mathrm{~V}_{1 / 3} \mathrm{O}_{3}$. En el centro se muestra un plano de $\mathrm{Cu}-\mathrm{V}$ representando la estructura de la red hexagonal. (b) Figura esquemática de la red formada por $\mathrm{Cu}^{2+}$ y los acoplamientos $J_{1}, J_{1}^{\prime}$ y $J_{2}$ entre los espines $\mathrm{Cu}^{2+}$.

estudiado por varios autores $[159,160]$. Este posee diferentes fases ordenadas a temperatura cero $[159,160]$ las cuales son mostradas en la figura (5.8). El diagrama de fases posee existe un punto particular tri-crítico en $J_{2}=J_{3}=\frac{J_{1}}{2}$ en donde todas las fases limitan. En este punto el hamiltoniano puede ser reescrito, salvo un término constante, como una suma sobre el espín total de hexágonos de la forma

$$
H=\operatorname{cte}+\frac{J_{1}}{2} \sum_{\gamma}\left|\mathbf{S}_{\gamma}\right|^{2},
$$

donde $\mathbf{S}_{\gamma}=\sum_{k \in \gamma} \mathbf{S}_{k}$ es el espín total en el hexágono $\gamma$. En este punto particular cualquier estado con espín total cero por hexágono es un estado fundamental clásico, dando como resultado un número macroscópico de configuraciones para estado fundamental. Esta clase de degeneración es análoga a que sucede en la red cuadrada $J_{1}-J_{2}$ para el caso $J_{2}=J_{1} / 2$ que ha sido extensamente estudiado mediante muchas técnicas como expansiones en serie [161, 162, 163, 164, 165, 166, 167], diagonalización exacta [168, 169, 170] y Montecarlo Cuántico [171, 172, 173]. Motivados por esta analogía, vamos a estudiar de manera exhaustiva la región del diagrama de fases $J_{2}=J_{3}$ que contiene el punto tri-crítico $J_{2}=J_{3}=J_{1} / 2$ con el objetivo de comprender la naturaleza del estado fundamental del modelo cuántico (5.14).

\subsubsection{Aspectos clásicos y semi-clásicos}

En esta sección estudiamos el límite clasico del modelo (5.14) y posteriormente, utilizando el mapeo a bosones de Holsetin-Primakoff en la aproximación lineal, adicionamos fluctuaciones cúanticas al problema clásico con el objetivo de analizar la estabilidad de las diferentes fases del estado fundamental cuántico a temperatura cero.

Para comenzar introducimos una representación de un espín clásico como

$$
\vec{S}_{\vec{R}, \alpha}=S\left(\cos \left(\vec{Q} \cdot \vec{R}+\phi_{\alpha}\right) \check{e}_{1}+\sin \left(\vec{Q} \cdot \vec{R}+\phi_{\alpha}\right) \check{e}_{2}\right)
$$




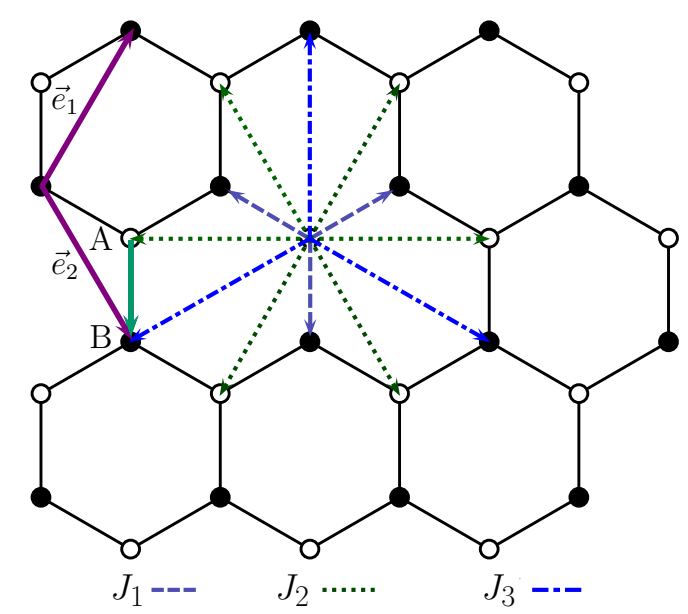

Figura 5.7: La red hexagonal formada por dos subredes: $A$ y $B$. Los vectores $\vec{e}_{1}=\left(\frac{\sqrt{3}}{2}, \frac{1}{2}\right)$ y $\vec{e}_{2}=\left(\frac{\sqrt{3}}{2},-\frac{1}{2}\right)$ son los vectores primitivos de la red directa y $\alpha=(0,-1)$ es el vector interno de la celda unidad formado por dos sitios: uno "tipo" A y otro "tipo" B. $J_{1}, J_{2}$ y $J_{3}$ son los acoplamientos antiferromagnéticos a primeros, segundos y terceros vecinos.

donde $\vec{R}$ es el vector posición de la celda unidad, $\vec{e}_{1}, \vec{e}_{2}$ son los vectores primitivos de la red directa y $\alpha=A, B$ es un índice interno en cada sub-red (ver figura (5.7)). El vector $\vec{Q}$ llamado vector de ordenamiento y de la fase interna $\phi_{\alpha}$ determinan el tipo orden del estado fundamental. Utilizando la ecuación (5.16) en el hamiltoniano (5.14) se obtiene la energía clásica

$$
\varepsilon\left(J_{1}, J_{2}, \vec{Q}, \phi_{A}, \phi_{B}\right),
$$

cuyo mínimo global determina los parámetros $\vec{Q}, \phi_{A}, \phi_{B}$. La soluciones muestran que para $0 \leq J_{2} / J_{1}<0.5$ el estado fundamental presenta una fase de Néel, con vector de ordenamiento $\vec{Q}=(0,0)$ y $\phi_{A}-\phi_{B}=\pi$. La configuración de espines se corresponde con dos subredes triangulares ordenadas paralelamentes como en la figura (5.9); para $J_{2} / J_{1}>0.5$ el estado fundamental posee una fase colineal con $\vec{Q}=(2 \pi \sqrt{3}, 0)$ y $\phi_{A}-\phi_{B}=\pi$. El ordenamiento consiste de cadenas zig-zag ordenadas paralelamentes acopladas de manera antiparalelas ( ver figura (5.9)). El punto $J_{2} / J_{1}=0.5$ que separa ambas fases corresponde a una transición de fase de primer orden debido a que la transición entre ambas fases se produce por un cruce niveles.

Usando la teoría de ondas de espín lineal desarrollada en la sección 2.2.1 adicionamos fluctuaciones cuánticas y estudiamos la estabilidad de las fases clásicas. Antes de utilizar el mapeo entre operadores de espín y operadores bosónicos se debe realizar una rotación de los operadores de espín en cada una de las fases ordenadas. Así para $0 \leq J_{2} / J_{1}<0.5$ realizamos una rotación en $\pi$ de una de las subredes,

$$
\begin{aligned}
& S_{\vec{r}_{i}}^{x} \rightarrow S_{\vec{r}_{i}}^{x}=\tilde{S}_{\vec{r}_{i}}^{x}, \\
& S_{\vec{r}_{i}}^{y} \rightarrow S_{\vec{r}_{i}}^{y}=-\tilde{S}_{\vec{r}_{i}}^{y}, \\
& S_{\vec{r}_{i}}^{z} \rightarrow S_{\vec{r}_{i}}^{z}=-\tilde{S}_{\vec{r}_{i}}^{z}
\end{aligned}
$$




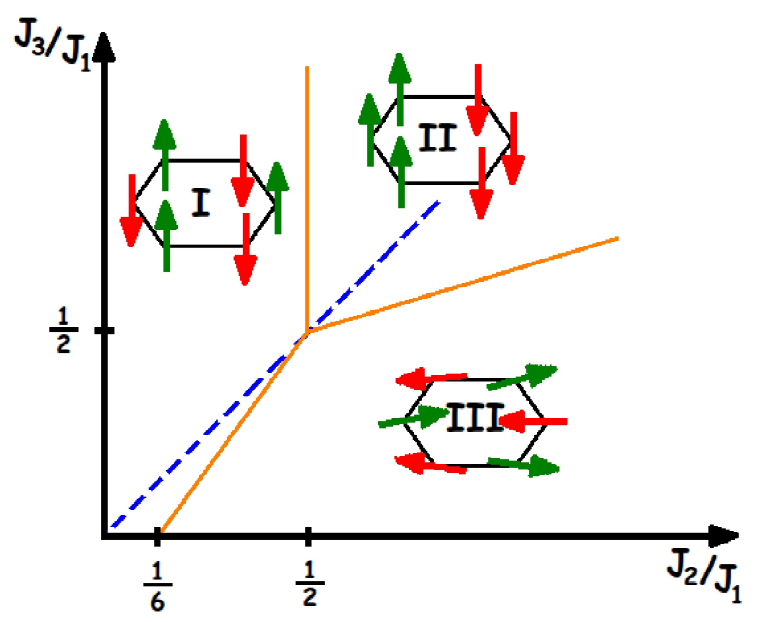

Figura 5.8: Diagrama de fases clásico del modelo (5.14). Hay tres fases diferentes esquematizadas: La fase de Néel (I) tiene dos (antiparalelos) subredes triangulares (esferas azules y rojos), la fase colineal (II) donde las cadenas antiferromagnéticas se encuentran antiparalelas; y la fase espiral (III). Nos enfocamos en la línea entrecortada correspondiendo a $J_{2}=J_{3}$ que incluye el punto de gran interés $\left(J_{2}=J_{3}=J_{1} / 2\right)$ donde el estado fundamental es infinitamente degenerado.

con $i \in$ sub-red B; mientras que para $J_{2} / J_{1}>0.5$ la rotación se aplica sobre filas enteras de espines de manera alternada (ver figura 5.9).

$$
\begin{aligned}
& S_{\vec{r}_{i}}^{x} \rightarrow S_{\vec{r}_{i}}^{x}=\tilde{S}_{\vec{r}_{i}}^{x}, \\
& S_{\vec{r}_{i}}^{y} \rightarrow S_{\vec{r}_{i}}^{y}=-\tilde{S}_{\vec{r}_{i}}^{y}, \\
& S_{\vec{r}_{i}}^{z} \rightarrow S_{\vec{r}_{i}}^{z}=-\tilde{S}_{\vec{r}_{i}}^{z}
\end{aligned}
$$

El parámetro de orden $m$ en la fase de Néel corresponde a la magnetización alternada; mientras que en la fase colineal corresponde a la magnetización total en las direcciones "zig-zag" y magnetización alternada en la dirección perpendicular.

$$
\begin{aligned}
m_{\text {Néel }} & =\left\langle\sum_{i \in A} S_{\vec{r}_{i}}^{z}-\sum_{j \in B} S_{\vec{r}_{j}}^{z}\right\rangle \\
m_{\text {Colineal }} & =\left\langle\sum_{i \in \text { cadena } \uparrow} S_{\vec{r}_{i}}^{z}-\sum_{j \in \text { cadena } \downarrow} S_{\vec{r}_{j}}^{z}\right\rangle
\end{aligned}
$$

Utilizando el mapeo de operadores de espín a operadores bosónicos de Holstein-Primakoff mostrada en la sección 2.2.1, y manteniendo solo los términos al orden $(1 / S)$ se obtiene un hamiltoniano cuadrático bosónico dado por

$$
\mathcal{H}=\epsilon_{0}+S \sum_{\mathbf{k} \in B Z} \psi_{\mathbf{k}}^{\dagger} \cdot M(\mathbf{k}) \cdot \psi_{\mathbf{k}}
$$




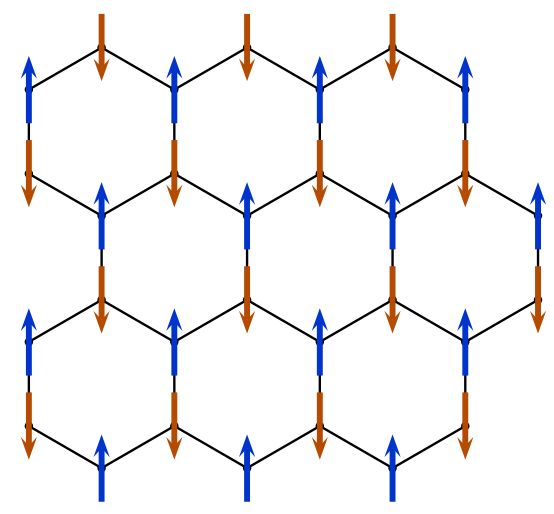

Fase Néel

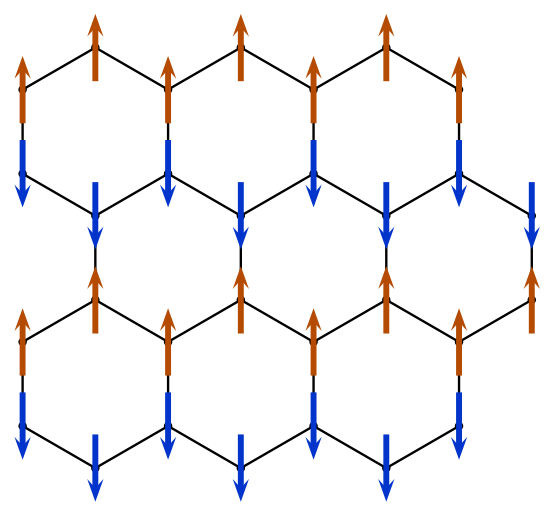

Fase Colineal

Figura 5.9: Figuras que representan los ordenes clásicos: Fase de Néel (izquierda); fase colineal (derecha).

donde $\epsilon_{0}$ es una función de $J_{1}, J_{2}$ y corresponde a la energía clásica. En la región $0<J_{2} / J_{1}<$ 0.5 tenemos

$$
\begin{aligned}
M(\mathbf{k})= & \frac{3}{2}\left(\begin{array}{cccc}
d_{N}(\mathbf{k}) & 0 & 0 & \gamma_{13}^{*}(\mathbf{k}) \\
0 & d_{N}(\mathbf{k}) & \gamma_{13}(\mathbf{k}) & 0 \\
0 & \gamma_{13}^{*}(\mathbf{k}) & d_{N}(\mathbf{k}) & 0 \\
\gamma_{13}(\mathbf{k}) & 0 & 0 & d_{N}(\mathbf{k})
\end{array}\right), \\
d_{N}(\mathbf{k})= & J_{1}+J_{2}\left(\gamma_{2}(\mathbf{k})+\gamma_{2}^{*}(\mathbf{k})-1\right) \\
\gamma_{2}(\mathbf{k})= & \frac{J_{1}}{3}\left(e^{-i \sqrt{3} k_{x}}+e^{\frac{i}{2}\left(\sqrt{3} k_{x}-3 k_{y}\right)}+e^{\frac{i}{2}\left(\sqrt{3} k_{x}+3 k_{y}\right)}\right) \\
\gamma_{13}(\mathbf{k})= & \frac{J_{1}}{3}\left(e^{\frac{i}{2}\left(k_{y}-\sqrt{3} k_{x}\right)}+e^{\frac{i}{2}\left(k_{y}+\sqrt{3} k_{x}\right)}+e^{-i k_{y}}\right) \\
& +\frac{J_{2}}{3}\left(e^{-i\left(k_{y}+\sqrt{3} k_{x}\right)}+e^{i\left(\sqrt{3} k_{x}-k_{y}\right)}+e^{2 i k_{y}}\right)
\end{aligned}
$$

mientras que en la región $0.5<J_{2} / J_{1}$ tenemos

$$
\begin{aligned}
& M(\mathbf{k})=\frac{1}{2}\left(\begin{array}{cccc}
d_{C}(\mathbf{k}) & h^{*}(\mathbf{k}) & \gamma(\mathbf{k}) & \xi^{*}(\mathbf{k}) \\
h(\mathbf{k}) & d_{C}(\mathbf{k}) & \xi(\mathbf{k}) & \gamma(\mathbf{k}) \\
\gamma(\mathbf{k}) & \xi^{*}(\mathbf{k}) & d_{C}(\mathbf{k}) & h^{*}(\mathbf{k}) \\
\xi(\mathbf{k}) & \gamma(\mathbf{k}) & h(\mathbf{k}) & d_{C}(\mathbf{k})
\end{array}\right) \\
& d_{C}(\mathbf{k})=-J_{1}+5 J_{2}+2 J_{2} \cos \left(\sqrt{3} k_{x}\right) \\
& h(\mathbf{k})=e^{\frac{i}{2}\left(-\sqrt{3} k_{x}+k_{y}\right)}+e^{\frac{i}{2}\left(\sqrt{3} k_{x}+k_{y}\right)} \\
& \gamma(\mathbf{k})=J_{1} \cos \left(\frac{\sqrt{3} k_{x}+3 k_{y}}{2}\right)+2 J_{2} \cos \left(\frac{\sqrt{3} k_{x}-3 k_{y}}{2}\right) \\
& \xi(\mathbf{k})=J_{1} e^{-i k_{y}}+J_{2}\left(e^{-i\left(\sqrt{3} k_{x}+k_{y}\right)}+e^{i\left(\sqrt{3} k_{x}-k_{y}\right)}+e^{2 i k_{y}}\right)
\end{aligned}
$$


Siguiendo los pasos desarrollados en el apéndice B diagonalizamos mediante una transformación paraunitaria el hamiltoniano cuadrático (5.22) obteniendo las relaciones de dispersión de los bosones

$$
\begin{aligned}
& \text { Fase de Néel } \\
w(\mathbf{k})=\frac{3}{2}\left[\left(J_{1}+J_{2}\right)^{2}-\left|\gamma_{13}(\mathbf{k})\right|^{2}+2 J_{2}\left(J_{1}+J_{2}\right)\left(-2 J_{1}+2 \operatorname{Re}\left(\gamma_{2}(\mathbf{k})\right)\right)\right. & \\
& \left.+J_{2}^{2}\left(-2 J_{1}+2 \operatorname{Re}\left(\gamma_{2}(\mathbf{k})\right)\right)^{2}\right]^{1 / 2} \\
w_{ \pm}(\mathbf{k})= & \frac{1}{2}\left\{d(\mathbf{k})^{2}+|h(\mathbf{k})|^{2}-\gamma(\mathbf{k})^{2}-|\xi(\mathbf{k})|^{2}\right. \\
\pm & {\left[\left(h^{*}(\mathbf{k}) \xi(\mathbf{k})\right)^{2}+h^{*}(\mathbf{k})\left(4 d(\mathbf{k})^{2} h(\mathbf{k})-2 h(\mathbf{k})|\xi(\mathbf{k})|^{2}-4 d(\mathbf{k}) \gamma(\mathbf{k}) \xi(\mathbf{k})\right)\right.} \\
+ & \left.\left.\xi^{*}(\mathbf{k})\left(\xi^{*}(\mathbf{k}) h^{2}(\mathbf{k})-4 d(\mathbf{k}) h(\mathbf{k}) \gamma(\mathbf{k})+4 \gamma^{2}(\mathbf{k}) \xi(\mathbf{k})\right)\right]^{1 / 2}\right\}^{1 / 2}
\end{aligned}
$$

En la figura (5.10) se muestra el diagrama de fases del estado fundamental en la aproximación de ondas de espín lineal en función de la magnitud del espín $S$ y la relación de acoplamientos $J_{2} / J_{1}$. El hecho de considerar a la magnitud del espín como una variable continua $S$ se debe a que de esta manera podemos explorar la transición desde el régimen clásico, correspondiente a $S \rightarrow \infty$, hasta el límite cuántico extremo $S=1 / 2$.

La linea púrpura a trazos determina las regiones de estabilidad de las fases ordenadas. Para un dado valor de $J_{2} / J_{1}$ existe un valor de espín $S_{c}$ tal que para el modelo con espín $S>S_{c}$ la fase ordenada es estable, mientras que para $S<S_{c}$ las fluctuaciones cuánticas son suficientemente grandes como para destruir el orden. Los bordes de las fases ordenadas se obtienen encontrando el valor de $J_{2} / J_{1}$ (y del espín $S$ ) para el cual el parámetro de orden se anula. Los resultados muestran que las fluctuaciones reducen notablemente la estabilidad de la fase de Néel alrededor de $J_{2} / J_{1}=1 / 2$. Para el caso de espín $S=1 / 2$, el orden de Néel es estable (al menos dentro de la aproximación lineal de ondas de espín) para $0<J_{2} / J_{1} \lesssim 0.29$, mientras que el orden colineal se muestra estable para $0.55 \lesssim J_{2} / J_{1}$. En la región intermedia $0.29 \lesssim J_{2} / J_{1} \lesssim 0.55$ las fluctuaciones cuánticas son lo suficientemente grandes (al menos al orden lineal en $1 / S$ ) como para desestabilizar el orden magnético dando la posibilidad a una fase desordenada. Sin embargo, el método de ondas de espín lineal no permite analizar la estructura de tal fase. Este tema es abordado en la siguiente sección utilizando la técnica de bosones de Schwinger y diagonalización exacta.

\subsubsection{Método de bosones de Schwinger}

En esta sección presentamos el estudio realizado el hamiltoniano cuántico (5.14) utilizando el método de bosones de Schwinger en la aproximación de campo medio desarrollado en la sección 2.2.2. La ventaja que tiene el utilizar éste método consiste que a diferencia de la aproximación de ondas de espín lineal, que considera correcciones cuánticas respecto de un estado clásico ordenado, el método de bosones de Schwinger no considera a priori ningún 


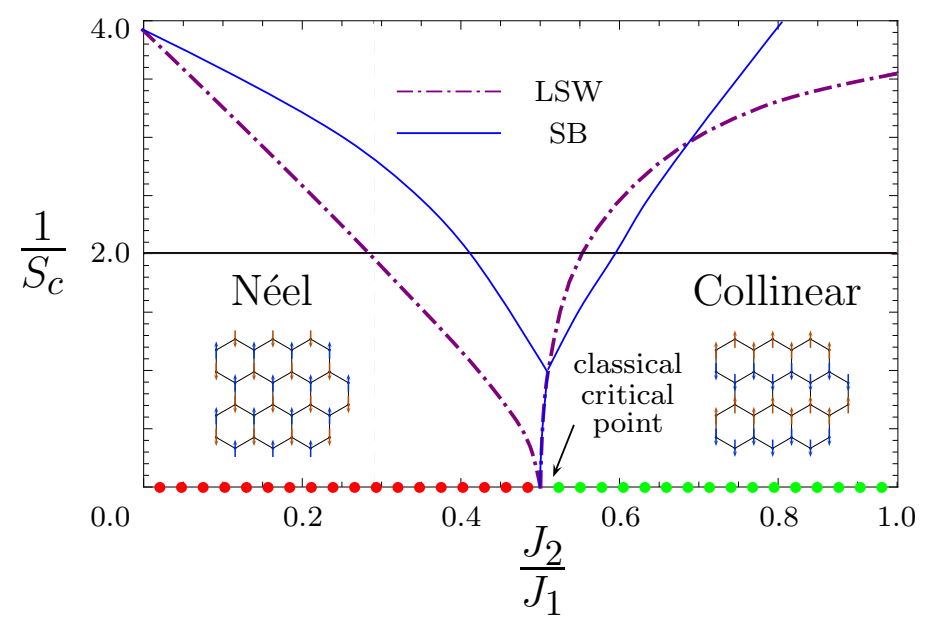

Figura 5.10: Inversa del espín crítico $S_{c}$ como función de $J_{2}$ : La línea de trazos y puntos muestra el borde obtenido que separa las regiones ordenadas y desordenadas, obtenidas por medio del método de ondas de espín lineal. Por encima de esta línea, las fluctuaciones cuánticas destruyen el orden magnético, y la magnetización alternada se anula. Para $S=1 / 2$ (línea llena negra) hay un pequeño rango $0.29 \lesssim J_{2} / J_{1} \lesssim 0.55$ donde no hay orden magnético. La línea llena azul corresponde al borde obtenido mediante bosones de Schwinger. Para el caso $S=1 / 2$, existe un rango $0.41 \lesssim J_{2} / J_{1} \lesssim 0.6$ donde el sistema tiene un gap de espín indicando la presencia de una fase desordenada (ver figura (5.13)). La línea punteada corresponde al límite clásico $S \rightarrow \infty$ donde el estado fundamental presenta orden de Néel para $J_{2} / J_{1}<0.5$, mientras que colineal para $J_{2} / J_{1}>0.5$.

tipo de estado inicial. Los pasos para obtener el hamiltoniano de campo medio no son mostrados de manera detallada siendo equivalentes a los desarrollados en la sección 5.1.2. Luego de utilizar el mapeo a bosones de Schwinger y el desacople de campo medio, el hamiltoniano puede ser reescrito como

$$
\mathcal{H}_{M F}=\epsilon_{o}+\sum_{\mathbf{k}} \mathbf{b}^{\dagger}(\mathbf{k}) \cdot M(\mathbf{k}) \cdot \mathbf{b}(\mathbf{k})
$$

donde $\epsilon_{0}$ está dado por

$$
\begin{aligned}
\epsilon_{0}= & J_{1} N_{c} \sum_{i=1}^{3}\left(A_{p, i}^{2}-B_{p, i}^{2}\right)+J_{2} N_{c} \sum_{i=1}^{6}\left(A_{s, i}^{2}-B_{s, i}^{2}\right)+J_{2} N_{c} \sum_{i=1}^{3}\left(A_{t, i}^{2}-B_{t, i}^{2}\right) \\
& -N_{c}(2 S+1)\left(\lambda_{A}+\lambda_{B}\right)
\end{aligned}
$$

y $M(\mathbf{k})$ es una matriz hermítica cuyos elementos dependen de los parámetros de campo medio $A^{\prime} s$ y $B^{\prime} s$ y de los multiplicadores de Lagrange $\lambda_{A, B}$ a ser determinados autoconsistentemente. En la expresión (5.28) $N_{c}$ es el número de celdas en la red, los índices $p, s, t$ etiquetan los parámetros de campo medio a primeros, segundos y terceros vecinos respectivamente, y $\lambda_{A}$, $\lambda_{B}$ son los multiplicadores de Lagrange introducidos para fijar el número de bosones en cada 
sub red ${ }^{4}$. Tomando el valor medio de los operadores $\mathbf{A}$ y $\mathbf{B}$ obtenemos

$$
\begin{aligned}
& A_{\alpha, a}=\left\langle\mathbf{A}_{\mathbf{r}_{i}, \mathbf{r}_{j}}\right\rangle \\
& B_{\alpha, a}=\left\langle\mathbf{B}_{\mathbf{r}_{i}, \mathbf{r}_{j}}\right\rangle
\end{aligned}
$$

donde los sitios $\mathbf{r}_{i}, \mathbf{r}_{j}$ conectan sitios a primeros, segundos y terceros vecinos (respectivamente $\alpha=p, s, t)$ en las direcciones correspondientes $a=1,2,3$ (primeros y terceros vecinos) y $a=1, \ldots, 6$ (segundos vecinos) como se muestra en la figura (5.7).

Estas ecuaciones deben ser resueltas de manera autoconsistente junto con la ecuación que fija el número de bosones en cada sitio

$$
\frac{1}{N_{c}} \sum_{\mathbf{r}_{i}, \sigma}\left\langle b_{\mathbf{r}_{i}, \sigma}^{\dagger} b_{\mathbf{r}_{i}, \sigma}\right\rangle=2 S
$$

donde $N_{c}$ es el número total de celdas. Las ecuaciones (5.29) y (5.30) involucran resolver un sistema 24 ecuaciones acopladas para los parámetros $A^{\prime} s$ y $B^{\prime} s$, más las ecuaciones de ligadura para determinar los valores de $\lambda^{\alpha}$. El análisis numérico fue realizado para sistemas finitos de hasta 3200 sitios y posteriormente extrapolados al límite termodinámico.

\subsubsection{Resultados de la teoría de campo medio.}

El procedimiento autoconsistente consiste en proponer valores iniciales para los parámetros $A^{\prime} s$ y $B^{\prime} s$ entonces determinar los nuevos parámetros a partir de las ecuaciones (5.29) y (5.30) hasta que la energía y los parámetros convergen. Después de que alcanzar la convergencia del método, se pueden calcular varias cantidades de interés como la energía, la correlación espín-espín y el espectro de excitaciones. Con esta información realizamos un diagrama de fases tentativo del estado fundamental.

A partir de los valores obtenidos de los parámetros $A^{\prime} s$ y $B^{\prime} s$ se observa una imagen similar a las fases clásicas como en la figura (5.9). Es decir, para cada par de sitios con una configuración $\uparrow \uparrow$ los parámetros $B^{\prime} s$ son un orden de magnitud mayor que los parámetros $A^{\prime} s$ indicando la presencia de orden ferromagnético, mientras que para una configuración de la forma $\downarrow \uparrow$ se observa el caso contrario revelando orden antiferromagnético.

Para soportar los resultados analíticos obtenidos para sistemas de muchos sitios, hemos realizado cálculos mediante diagonalización exacta del hamiltoniano de espín original (5.14) para $N=18,24$ y 32 sitios con condiciones de contorno periódicas para el caso $S=1 / 2$ utilizando las librerías de SPINPACK [65]. En la figura (5.11-izquierda) se muestra la energía del estado fundamental por celda unidad como función de la frustración para $N=32$ sitios, calculado por medio de SBMFT y diagonalización exacta. Es interesante notar el buen acuerdo que tienen los resultados obtenidos mediante diagonalización exacta y de la teoría de campo

\footnotetext{
${ }^{4} \mathrm{~A}$ diferencia del caso de la red de kagomé estudiada en la primera parte del capítulo permitimos que los parámetros de campo medio puedan tomar diferentes valores según la dirección en la red y además introducimos un multiplicador de Lagrange por cada subred $\lambda_{A, B}$.
} 

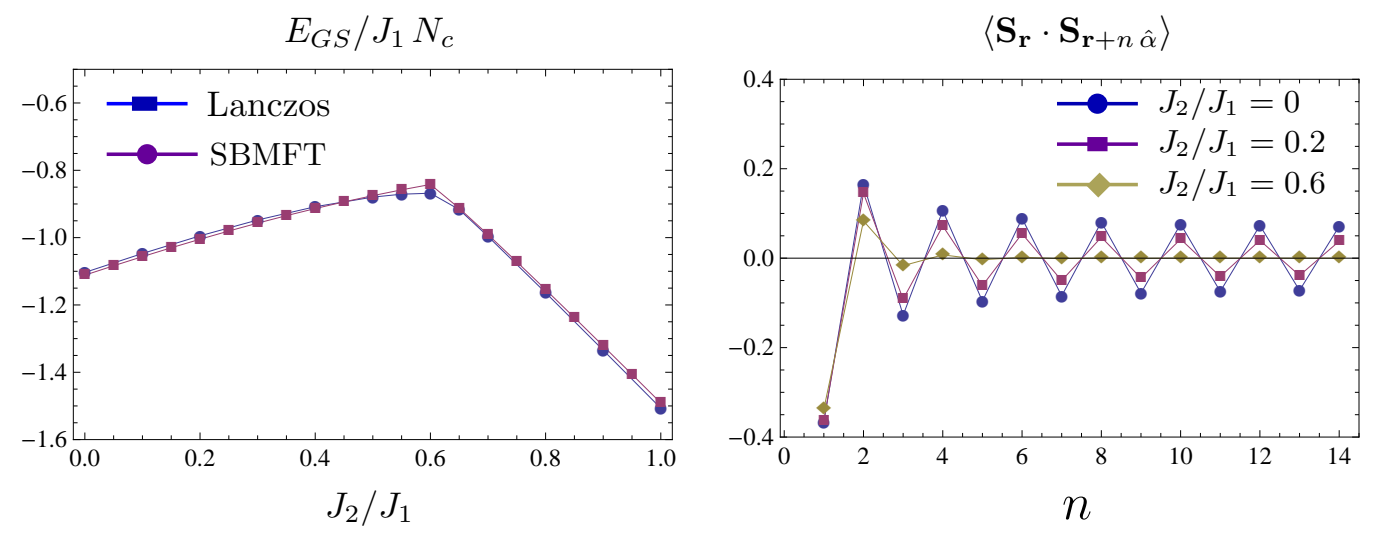

Figura 5.11: (a): Energía del estado fundamental por celda unidad como función de $J_{2} / J_{1}$ para una red de 32 sitios. Los círculos son resultados exactos (lanczos) y los cuadrados son los resultados de SBMFT. (b): Correlación espín-espín vs distancia $X$ en la dirección zig-zag obtenida mediante. Para $0<J_{2} / J_{1} \lesssim 0.41$, la correlación espín-espín acuerda con una fase de Néel con orden de largo alcance (LRO); para $0.41 \lesssim J_{2} / J_{1} \lesssim 0.6$ las correlaciones son de corto alcance indicando una zona de con gap en la excitaciones y orden de corto alcance (SRO).

medio. En la figura (5.12) se muestra el módulo de los parámetros de campo medio antiferromagnéticos a primeros vecinos como función de $J_{2} / J_{1}$. En (5.12) se comparan los parámetros de campo medio $|A|$ a primeros vecinos calculados mediante diagonalización exacta y por medio de la teoría de campo medio. Ambos resultados muestran un comportamiento similar en la zona de la transición: una discontinuidad abrupta para un valor determinado de $J_{2} / J_{1}$ (el mismo comportamiento presentan los parámetros ferromagnéticos $|B|$ no mostrado aquí). Ésta discontinuidad indica la presencia de una transición de primer orden entre fases con diferentes simetrías.

En el límite termodinámico $N_{c} \rightarrow \infty$ un estado que posee orden de largo alcance se caracteriza, en el lenguaje de los bosones de Schwinger, por una condensación de los bosones en algún vector de onda $Q_{0}$. Esto implica que la relación de dispersión de los bosones en una fase ordenada no posee gap. Como mencionamos anteriormente las ecuaciones de autoconsistencia (5.29) y (5.30) se resuelven numéricamente para sistemas finitos, por lo tanto para detectar las regiones con gap calculamos éste como función de $J_{2} / J_{1}$ para diferentes tamaños y posteriormente realizamos un escaleo. La estrapolación del gap de las excitaciones como función de $J_{1} / J_{1}$ se muestra e la figura (5.13). En el rango $0.45<J_{2} / J_{1}<0.6$ el sistema presenta un gap finito indicando una región sin orden magnético.

En la figura (5.11-derecha) se muestran los resultados de las correlaciones espín-espín $\left\langle\mathbf{S}_{1} \mathbf{S}_{j}\right\rangle$ a lo largo de dos direcciones particulares. Para $0<J_{2} / J_{1} \lesssim 0.45$ las correlaciones presentan una estructura que se corresponde con fase ordenada tipo Néel, mientras que para $0.6 \lesssim J_{2} / J_{1}$ las correlaciones revelan orden ferromagnético en la dirección zig-zag correspondiendo a la fase colineal. El resultado más interesante se observa en la región intermedia $0.45 \lesssim J_{2} / J_{1} \lesssim 0.6$ donde los resultados son consistentes con la presencia de un gap. En esta 

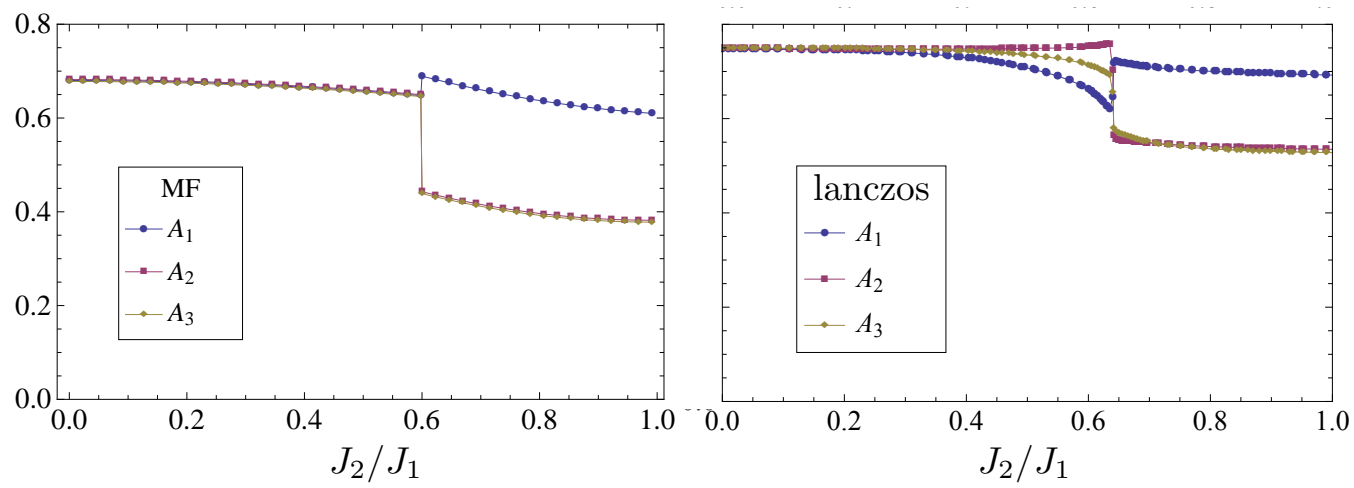

Figura 5.12: Parámetros de antiferromagnéticos $|A|$ vs $J_{2} / J_{1}$ para espín $S=1 / 2$, (derecha): diagonalización exacta $N=24$ sitios; (izquierda) campo medio. La discontinuidad de los parámetros cerca de $J_{2} / J_{1}=0.6$ da indicios de una transición de primer orden entre la fase desordenada y la fase colineal con orden de largo alcance.

región hemos encontrado correlaciones antiferromagnéticas de corto alcance. Como ejemplo, en la figura 5.11 se muestran las correlaciones para $J_{2} / J_{1}=0,0.2$ y 0.6 en la dirección zig-zag de la red.

Los resultados del análisis se resumen en la figura 5.10 que muestra el diagrama de fases del estado fundamental como función de $1 / S$ y $J_{2} / J_{1}$. Por un lado, para $1 / S$ más chico que una valor crítico $1 / S_{c}\left(J_{2} / J_{1}\right)$ el sistema posee orden de largo alcance caracterizado por una condensación de bosones en un vector de ordenamiento $Q_{0}$. Por otro lado, cuando $1 / S$ es mayor que $1 / S_{c}\left(J_{2} / J_{1}\right)$ el sistema no posee orden magnético. Del análisis anterior surge la siguiente pregunta: ¿La fase con gap, es desordenada o presenta algún tipo de orden distinto al magnético?. Para responder ésta pregunta, no es suficiente con la correlación espín-espín; es necesario calcular otra cantidad. Un tipo de orden que se puede presentar es el que se conoce como "orden de dímeros". El operador de dímero de un par de sitios $(i, j)$ está dado por (ver ecuación (1.63) sección 1.3.3)

$$
d_{\mathbf{r}_{i}, \mathbf{r}_{j}}=\frac{\left(1-P_{\mathbf{r}_{i}, \mathbf{r}_{j}}\right)}{2}
$$

donde $P_{i, j}=2\left(\mathbf{S}_{\mathbf{r}_{i}} \cdot \mathbf{S}_{\mathbf{r}_{j}}+1 / 4\right)$ es el operador de permutación de espín. Éste operador es mayor (menor) que 0.25 cuando la correlación de espín es negativa (positiva), igual a 1 en un singlete y a 0 en un triplete. La correlación "dímero-dímero" entre sitios $(i, j)$ y $(k, l)$ es

$$
p_{\left(\mathbf{r}_{i}, \mathbf{r}_{j}\right),\left(\mathbf{r}_{k}, \mathbf{r}_{l}\right)}=\frac{\left\langle d_{\mathbf{r}_{i}, \mathbf{r}_{j}} d_{\mathbf{r}_{k}, \mathbf{r}_{l}}\right\rangle-\left\langle d_{\mathbf{r}_{i}, \mathbf{r}_{j}}\right\rangle\left\langle d_{\mathbf{r}_{k}, \mathbf{r}_{l}}\right\rangle}{\left\langle d_{\mathbf{r}_{i}, \mathbf{r}_{j}}\right\rangle-\left\langle d_{\mathbf{r}_{i}, \mathbf{r}_{j}}\right\rangle\left\langle d_{\mathbf{r}_{k}, \mathbf{r}_{l}}\right\rangle}
$$

Si $p_{(i, j),(k, l)}=1$ la presencia de un dímero en el par $(i, j)$ implica la existencia de una dímero en el par $(k, l)$; si $p_{(i, j),(k, l)}=0$ los pares de sitios no están correlacionados. Si $p_{(i, j),(k, l)}$ es negativo, un singlete en el par $(i, j)$ induce una tendencia hacia una correlación ferromagnética en el par $(k, l)$. En la figura (5.14) se muestran las correlaciones espín-espín correspondientes a cada sector del diagrama de fases; en la figura (5.15) se muestra el patrón de correlaciones 


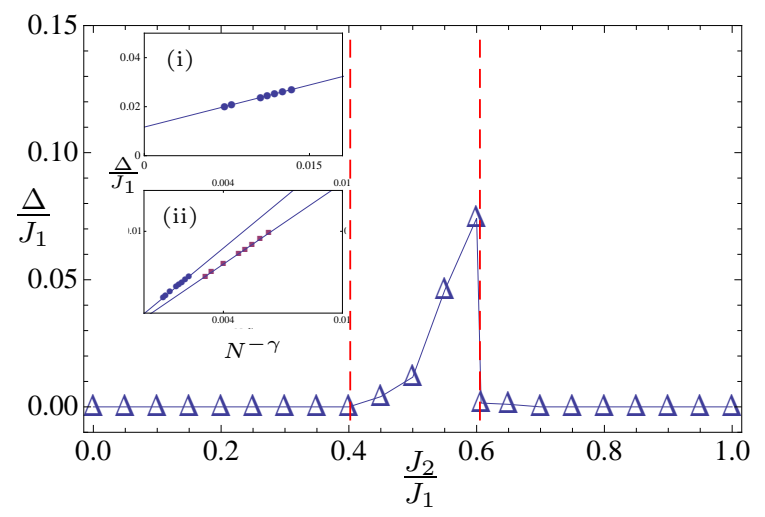

Figura 5.13: (a): Gap de las excitaciones en función de $J_{2} / J_{1}$ para $S=1 / 2$. En la región $J_{2} / J_{1} \sim 0.6$ el sistema permanece con gap. Recuadro: escaleo de tamaño finito para el gap. (i) $J_{2} / J_{1}=0.5(\gamma=0.6451)$, (ii): Los círculos corresponden a $J_{2} / J_{1}=0.05(\gamma=0.911)$ y los cuadrados corresponden a $J_{2} / J_{1}=0.35(\gamma=0.758)$.

para dímeros para $J_{2} / J_{1}=0.56$. Ambos cálculos fueron realizados mediante diagonalización exacta para $N=24$ sitios en $J_{2} / J_{1}=0.56$. Más allá de que el tamaño no es suficientemente grande como para ser concluyente, se puede observar que tanto las correlaciones espín-espín como las dímero-dímero decaen rápidamente con la distancia indicando desorden. Este resultado da indicios de que efectivamente la fase sin gap corresponde a una fase tipo líquido de espines ${ }^{5}$.

\subsubsection{Discusión y Conclusiones}

Para resumir, los resultados obtenidos con diferentes técnicas sugieren la existencia de una región (en el régimen de frustración intermedia) donde el sistema no muestra orden magnético para $S=1 / 2$. Por un lado, el análisis de ondas de espín lineal predice que la región con orden de Néel de largo alcance se extiende hasta $J_{2} / J_{1} \approx 0.29$ y la fase colineal antiferromagnética está presente hasta $J_{2} / J_{1} \gtrsim 0.55$. Por otro lado, los resultados con SBMFT predicen una región desordenada magnéticamente para $0.41 \lesssim J_{2} / J_{1} \lesssim 0.6$. En esta región, la relación de dispersión de los bosones presenta un gap y el análisis de las funciones de correlación espínespín revelan orden de corto alcance tipo Néel seguido por una fase colineal para $J_{2} / J_{1} \gtrsim 0.6$. Finalmente, el cálculo de las correlaciones dímero-dímero mediante la diagonalización exacta del hamiltoniano de espín para sistemas de $N=32$ sitios, muestra que la fase desordenada aún en discusión podría corresponder a un estado tipo valence bonds cristal.

\footnotetext{
${ }^{5}$ Este análisis está aún bajo discusión como parte de un trabajo en colaboración con el Dr. Daniel Cabra, Dr. Carlos Lamas, Dr. Piere Pujol.
} 


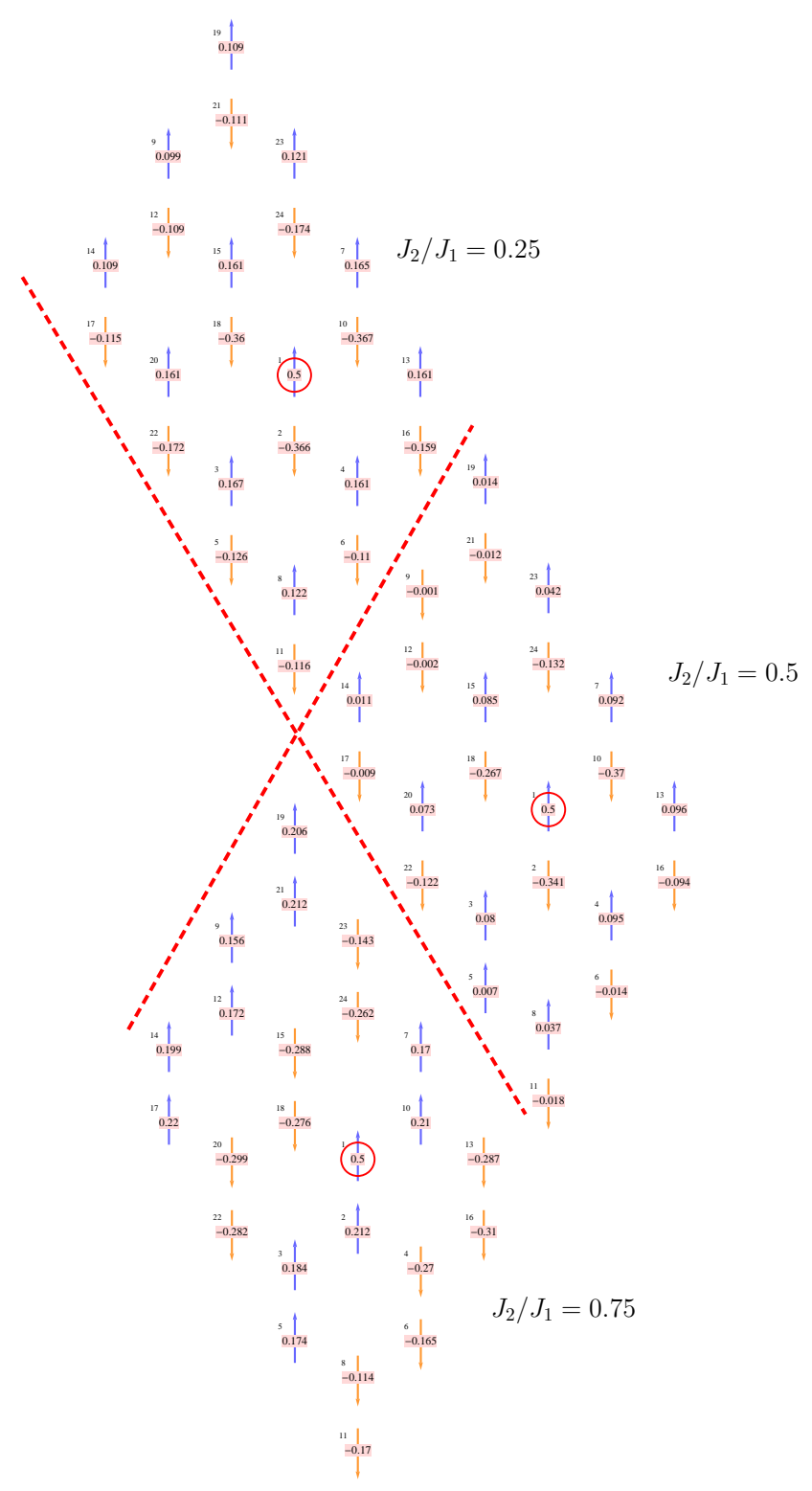

Figura 5.14: Correlación espín-espín, para tres valores particulares de $J_{2} / J_{1}$. (arriba) $J_{2} / J_{1}=$ 0.25: se observa la estructura tipo Néel. (centro) $J_{2} / J_{1}=0.5$ : la estructura es una mezcla Néel-colineal con correlaciones que van rápidamente a cero. (abajo) $J_{2} / J_{1}=0.75$ : se observa la estructura de la fase colineal con filas de espines ordenadas. 


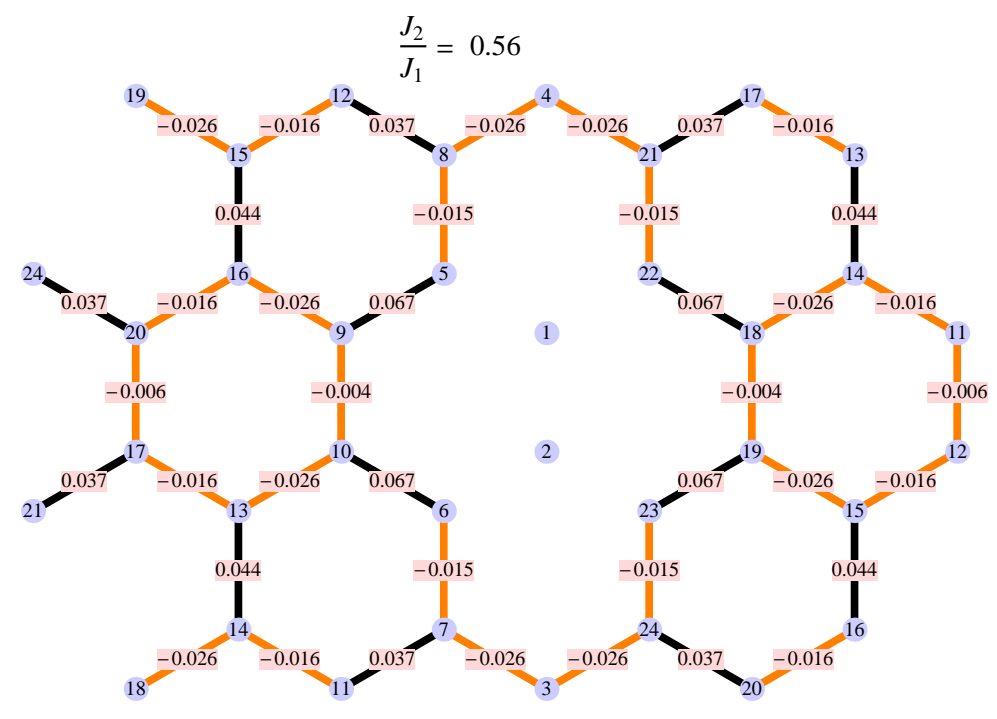

Figura 5.15: Correlación dímero-dímero, $p_{(1,2)(k, l)}$ para $J_{2} / J_{1}=0.56$ entre los pares de sitios $(1,2)$ y $(k, l)$ en el estado fundamental de $N=24$ sitios. Los números arriba de los links $(k, l)$ son los valores de $p_{(1,2)(k, l)}$. Las líneas negras (naranjas) indican valores positivos (negativos) de $p_{(1,2)(k, l)}$. 


\section{Capítulo 6}

\section{Conclusiones generales y perspectivas}

Finalizamos estas tesis con algunos comentarios generales sobre la metodología empleada y con un breve resumen de los principales resultados obtenidos.

\subsubsection{Técnicas empleadas.}

En el capítulo 2 presentamos algunas de las técnicas más utilizadas para estudiar sistemas fuertemente correlacionados en bajas dimensiones. La primera parte del capítulo, fue dedicada al método conocido como Bosonización que es una de las técnicas analíticas más utilizadas para estudiar sistemas fermiónicos interactuantes unidimensionales. Para poder estudiar los sistemas de espines $S=1 / 2$ en redes unidimensionales, estudiamos el mapeo de variables de espín a variables fermiónicas conocido como transformación de Jordan-Wigner. La segunda parte fue dedicada a los métodos analíticos que permiten estudiar sistemas en 2 y 3 dimensiones. En particular, nos centramos en dos métodos muy importantes: la teoría de "ondas de espín lineal" y la de "bosones de Schwinger", las cuales permiten estudiar distintos tipos de fases del estado fundamental del modelo de Heisenberg. La parte final del capítulo fue dedicada a la descripción de dos técnicas numéricas para tratar sistemas fuertemente correlacionados. Estas técnicas son Lanczos, que es un algoritmo que permite diagonalizar matrices de dimensiones muy grandes $\left(\right.$ ej. $\left.2^{36} \times 2^{36}\right)$; y DMRG, que es una técnica que permite estudiar el estado fundamental de sistemas unidimensionales de hasta centenas de sitios dependiendo del problema en cuestión.

\subsubsection{Modelos estudiados.}

En los capítulos 3, 4 y 5, presentamos el estudio de varios modelos para sistemas fuertemente correlacionados. En el capítulo 3, consideramos el modelo de Hubbard con interacciones electrónicas y acoplamientos con la red, para intentar describir las propiedades aisladoras del estado fundamental (a temperatura cero) de diversos compuestos orgánicos. En el capítulo 4, investigamos el modelo de Heisenberg de sistemas de espines frustrados magnéticamente, acoplados con los grados de libertad de la red. Analizamos las propiedades de las posibles fases magnetoelásticas del estado fundamental y como dependen éstas de la frustración y del 
acoplamiento con la red. Finalmente, en el capítulo 5, estudiamos las propiedades del modelo de Heisenberg en dos dimensiones en dos tipos de redes. En la primera parte estudiamos el estado fundamental del modelo de Heisenberg en la red de Kagomé con interacciones a primeros y terceros vecinos, revelando que la inclusión del acoplamiento a terceros vecinos estabiliza un orden magnético. En la segunda parte, analizamos el modelo de Heisenberg en la red hexagonal con interacciones a primeros, segundos y terceros vecinos. Nuestros resultados indican que la inclusión de frustración desestabiliza el orden magnético permitiendo la formación de una fase tipo líquido de espines en la región altamente degenerada del modelo clásico.

\subsubsection{Trabajos en ejecución y perspectivas futuras.}

Actualmente están en ejecución o próximos a iniciarse diversos estudios relacionados con los trabajos aquí presentados. Entre ellos merecen mencionarse los siguientes:

- Influencia de términos anarmónicos en el acoplamiento espín-fonón en cadenas de espines unidimensionales. Utilizando bosonización y simulaciones numéricas con DMRG, estudiamos el rol de los términos anarmónicos en la curva de magnetización.

- Estados desordenados en la red de Kagomé con interacciones bicuadráticas. Esto nos permitirá determinar la estabilidad de la fase desordenada del estado fundamental, y si la competencia de varias interacciones permite seleccionar distintos tipos de fases.

- Análisis de la curva de magnetización del modelo de Heisenberg en la red hexagonal. Esta extensión tiene como objetivo principal estudiar la posibilidad de determinadas fases desordenadas para valores racionales de magnetización. 


\section{Bibliografía}

[1] Metal-Insulator Transitions by N. Mott, (Taylor and Francis, London, 1997).

[2] C. Kittel Introduction to Solid State Physics, 6 ed. (J. Wiley, New York, 1986)

[3] N. W. Ashcroft and N. D. Mermin, Solid State Physics, (Thomson Learning, 1976)

[4] A. Auerbach, Interacting Electrons and Quantum Magnetism, 1st ed. (Springer-Verlag, New York, 1994)

[5] J.G. Bednorz and K.A. Muller, Z. Phys. B64, 188 (1986); M.K. Wu et. al., Phys. Rev. Lett. 58, 908 (1987).

[6] E. Dagotto, Rev. Mod. Phys. 66, 763 (1994).

[7] J. Hubbard, Proc. Roy. Soc. A 276, 238 (1963), J. Hubbard, Proc. Roy. Soc. A 277, 237 (1963), J. Hubbard, Proc. Roy. Soc. A 281, 401 (1964).

[8] Fabian H. L. Essler, Holger Frahm, Frank Göhmann, Andreas Klümper and Vladimir E. Korepin, The One-Dimensional Hubbard Model.

[9] E. Lieb and F. Wu, Phys. Rev. Lett. 20, 1445 (1968).

[10] H. A. Bethe, Z. Phys. 71, 205 (1931).

[11] W. Metzner and D. Vollhardt, Correlated lattice fermions in $d=\infty$ dimensions, Phys. Rev. Lett. 62 (1989) 324. Erratum: Phys. Rev. Lett 62 (1989) 1066.

[12] E. Muller-Hartmann, Z. Phys. B 74, 507 (1989).

[13] Yu. A. Izyumov, Usp. Fiz. Nauk. 165, 403 (1995) Phys. Usp. 38, 385 (1995).

[14] A. Georges, G. Kotliar, W. Krauth and M. Rozenberg, Rev. Mod. Phys. 68, 13 (1996).

[15] F. Gebhard, The Mott-Hubbard Metal Insulator Transition: Models and Methods (Springer, Berlin, 1997).

[16] S. G. Ovchinnikov, V. V. Valḱov, Hubbard operators in the theory of strongly correlated electrons (Imperial College Press, 2004). 
[17] A.H. MacDonald, S. M. Girvin, and D. Yoshioka, Phys. Rev. B 37 (1988) 9753

[18] T. Giamarchi, Quantum Physics in One Dimension (Oxford Science Publications, 2004).

[19] A. M. Tsvelik, Quantum Field Theory in Condensed Matter Physics (2nd Ed.) (Cambridge University Press, 2003).

[20] D. Pines and P. Nozieres, Theory of Quantum Liquids (Westview Press, 1999).

[21] H. J. Schulz, G. Cuniberti and P. Pieri, Fermi liquids and Luttinger liquids, in G. Morandi et al. eds, Field Theories for Low-Dimensional Condensed Matter Systems (Berlin:Springer Verlag, 2000). Chia Laguna Summer School, September 1997, preprint cond-mat/9807366.

[22] L. D. Landau, Sov. Phys. JETP 3, 920 (1957), L. D. Landau, Sov. Phys. JETP 5, 101 (1957), L. D. Landau, Sov. Phys. JETP 8, 70 (1958).

[23] Gordon Baym and Christoper Pethick, Landau Fermi-Liquid Theory. (WILEY-VCII Verlag GmbH \& Co. KGaA, 2004).

[24] L. Néel, Annales de physique (Paris) 18 (1932).

[25] N.D. Mermin and H.Wagner, Phys. Rev. Lett. 17, 1133 (1966).

[26] P. W. Anderson, Mater. Res. Bull. 8, 153 (1973); Science 235, 1196 (1987).

[27] N. D. Mermin and H. Wagner, Phys. Rev. Lett. 17, 1307 (1968).

[28] A. A. Belavin, A. M. Polyakov, and A. B. Zamolodchikov, Nucl. Phys. B 241, 333 (1984).

[29] . P. Di Francesco, P. Mathieu, and D. Sénéchal, Conformal Field Theory (SpringerVerlag, New York, 1997).

[30] A. O. Gogolin, A. A. Nersesyan and A. M. Tsvelik Bosonization and Strongly Correlated Systems, Cambridge, University Press (1998).

[31] M. Yamashita, T. Ishii, and H. Matsuzaka, Coord. Chem. Rev. 198, 347 (2000).

[32] P. Lemmens, G. Güntherodt, and C. Gros, Phys. Rep. 375, 1 (2003).

[33] J. Villian, J. Phys. C 10, 1717 (1977).

[34] G. Toulouse, Commun. Phys. 2, 115 (1977).

[35] D. A. Huse, V. Elser, Phys. Rev. Lett. 60, 2531 (1988); B. Bernu, C. Lhuillier, L. Pierre, Phys.Rev. Lett. 69, 2590 (1992); L. Capriotti, A. E. Trumper, S. Sorella, Phys. Rev. Lett. 82, 3899 (1999). 
[36] R. E. Peierls, Quantum Theory of Solids, chap.5, p. 109, (Oxford University Press, 1955).

[37] Para una descripción detallada, ver J.W. Bray et al. en . Extended linear chain

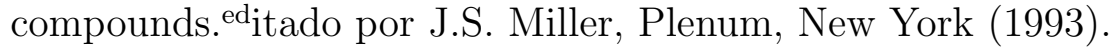

[38] M. Hase et al., Phys. Rev. Lett. 70, 3651(1993).

[39] T. Holstein, Ann. Phys. 8 (1959) 325.

[40] Sumathi Rao, Diptiman Sen An introduction to bosonization and aome of its applications, arXiv:cond-mat/0005492

[41] D. Sénéchal An introduction to bosonization, arXiv:cond-mat/9908262.

[42] Jan von Delft, Herbert Schoeller, Bosonization for Beginners - Refermionization for Experts, arXiv:cond-mat/9805275.

[43] X.-G. Wen, Int. J. Mod. Phys. B 6, 1711 (1992).

[44] P. Jordan and E. Wigner, Z. Phys.47, 631 (1928).

[45] See for instance D. C. Cabra and P. Pujol, in Quantum Magnetism, Lect. Notes Phys. 645 (2004).

[46] P. Lecheminant and E. Orignac, Phys. Rev. B 69, 174409 (2004).

[47] D. C. Mattis, The Theory of Magnetism, Springer-Verlag, Heidelberg (1988).

[48] N. Read, and D. M. Newns, J. Phys. C 16, L1055, (1983).

[49] N. Read and D. M. Newns, J. Phys. C 16, 3273 (1983).

[50] A. Auerbach, and K. Levin, Phys. Rev. Lett. 57, 877 (1986).

[51] Ian Affleck, Phys. Rev. Lett. 54, 966(1985).

[52] I. Affleck and J.B. Marston, Phys. Rev. B 37, 3774(1988).

[53] Y. Ran and X.G. Wen, cond-mat/0609620v3 (2006).

[54] D.P. Arovas and A. Auerbach, Phys. Rev. B 38, 316 (1988).

[55] N. Read and Subir Sachdev, Phys. Rev. Lett. 66, 1773 (1991).

[56] Rebecca Flint and P. Coleman, Physical Review B 79, 014424 (2009).

[57] Density-Matrix Renormalization, edited by I. Peschel, X. Wang, M. Kaulke, y K. Hallberg (Springer, Berlin, 1999).

[58] M. N. Barber, Vol. 8 dePhase Transitions and Critical Phenomena (Academic Press, London,1984). 
[59] S-K Ma,Modern Theory of Critical Phenomena (Addison Wesley Pub., 1997).

[60] C. Lanczos, J. Res. Nat. Bur. Stand. 45, 255 (1950).

[61] D. G. Pettifor e D. L. Weaire, The Recursion Method and Its Applications, Springer Series in Solid-State Sciences, Vol. 58 (Springer, Berlin/Heidelberg, 1985).

[62] S. R. White, Phys. Rev. Lett. 69, 2863 (1992); Phys. Rev. B 48, 10345 (1993).

[63] K. Hallberg, Adv. Phys. 55, 477 (2006); U. Schollwöck, Rev. Mod. Phys. 77, 259 (2005).

[64] http://alps.comp-phys.org.

[65] J. Schulenburg, program package SPINPACK, http://www-e.unimagdeburg.de/jschulen/spin/

[66] D. Jérome and H. J. Schulz, Adv. Phys. 31, 299 (1982). T. T. Ishiguro and K. Yamaji, Organic Superconductors, Vol. 88 of Springer-Verlag Series in Solid-State Science (Springer-Verlag, Berlin, Heidelberg, 1990).

[67] K.C. Ung, S. Mazumdar and D. Toussaint, Phys. Rev. Lett. 73, (1994) 2603.

[68] S. Mazumdar, S. Ramasesha, R.T. Clay and D.K. Campbell, Phys. Rev. Lett. 82, (1999) 1522.

[69] H. Seo and H. Fukuyama, J. Phys. Soc. Jpn. 66, (1997) 1249.

[70] H. Seo, J. Phys. Soc. Jpn. 69, (2000) 805.

[71] N. Kobayashi and M. Ogata, J. Phys. Soc. Jpn. 66, (1997) 3356.

[72] N. Kobayashi, M. Ogata and K. Yonemitsu, J. Phys. Soc. Jpn. 67, (1998).

[73] J. Riera and D. Poilblanc, Phys. Rev. B 59, (1999) 2667.

[74] J. Riera and D. Poilblanc, Phys. Rev. B 62, (2000) R16243.

[75] K. Hiraki and K. Kanoda, Phys. Rev. Lett. 80, (1998) 4737.

[76] S. Mazumdar, D. Campbell, R.T. Clay and S. Ramasesha, Phys. Rev. Lett. 82, (1999) 2411.

[77] K. Hiraki and K. Kanoda, Phys. Rev. Lett. 82, (1999) 2412.

[78] N. Biskup, J.A.A.J. Perenboom, J.S. Brooks, and J.S. Qualls, Solid State Comm. 107, (1998) 503.

[79] D.S. Chow, F. Zamborszky, B. Alavi, D.J. Tantillo, A. Baur, C.A. Merlic, and S.E. Brown, Phys. Rev. Lett. 85, (2000) 1698. 
[80] F. Nad, P. Monceau, C. Carcel and J.M. Fabre, J. Phys. Cond. Matt. 12, (2000) L435.

[81] F. Nad, P. Monceau, C. Carcel, and J.M. Fabre, Phys. Rev. B 62, (2000) 1753.

[82] P. Monceau, F.Y. Nad, and S. Brazovskii, Phys. Rev. Lett. 86, (2001) 4080.

[83] K. Miyagawa, A. Kawamoto and K. Kanoda, Phys. Rev. B 62, (2000) R7679.

[84] H. Seo, J. Merino, H. Yoshioka and M. Ogata, J.Phys. Soc. Jap. 75, 051009 (2006).

[85] R.T. Clay, S. Mazumdar and D.K. Campbell, Phys. Rev. B 67, (2003) 115121.

[86] D.C. Cabra, A. De Martino, A. Honecker, P. Pujol, and P. Simon, Phys. Rev. B 63, (2001) 094406.

[87] G. Delfino and G. Mussardo, Nucl. Phys. B 516, (1998) 675.

[88] M. Fabrizio, A.O. Gogolin, and A.A. Nersesyan, Nucl. Phys. B 580, 647 (2000).

[89] Z. Bajnok, L. Palla, G. Takacs, and F. Wagner, Nucl. Phys. B 601, (2001) 503.

[90] G.Z. Tóth, J. Phys. A 37, 9631 (2004).

[91] H.D. Rosales, D.C. Cabra, M.D. Grynberg, G.L. Rossini, and T. Vekua, Phys. Rev. B 75, (2007) 174446.

[92] H.D. Rosales and G.L. Rossini, Phys. Rev. B 76, (2007) 224404.

[93] K. Hida and I. Affleck, J. Phys. Soc. Jpn. 74, (2005) 1849.

[94] C.K. Majumdar and D. K. Ghosh, J. Math. Phys. 10, 1399 (1969).

[95] F. D. M. Haldane, Phys. Rev. B 25, 4925 (1982).

[96] T. Tonegawa and I. Harada, J. Phys. Soc. Jpn. 56, 2153 (1987);

[97] K. Okamoto and K. Nomura, Phys. Lett. A 169, 433 (1992).

[98] D. Allen and D. Sénéchal, Phys. Rev. B 51, 6394 (1995).

[99] R. Chitra, S. Pati, H. R. Krishnamurthy, D. Sen, and S. Ramasesha, Phys. Rev. B 52, 6581 (1995).

[100] S. R. White and I. Affleck, Phys. Rev. B 54, 9862 (1996).

[101] M. Matsuda and K. Katsumata, J. Mag. Mag. Mat. 140-144,1671 (1995).

[102] H. Kikuchi, H. Nagasawa, Y. Ajiro, T. Asano, and T. Goto, Physica B 284-288, 1631 (2000).

[103] Y. Hosokoshi, K. Katoh, K. Inoue, and T. Goto, J. Phys. Soc. Jpn. 68, 2910(1999). 
[104] M. Hagiwara, Y. Narumi, K. Kindo, N. Maeshima, K. Okunishi, T. Sakai, and M. Takahashi, Physica B 294-295, 83 (2001); N. Maeshima M. Hagiwara, Y. Narumi, K. Kindo, N. Maeshima, K. Okunishi, cond-mat/0208373.

[105] T. Tonegawa and I. Harada, Physica B 155, 379 (1989).

[106] M. Schmidt, C. Gerhardt, K.-H. Mütter and M. Karbach, J. Phys. Condens. Matter. 8, 553 (1996).; C. Gerhard, A. Fledderjohann, E. Aysal, K-H. Mütter, J.F. Audet, and H. Krö̈er, ibid 9, 3435 (1997)

[107] D.C. Cabra, A. Honecker, and P. Pujol, Eur. Phys. J. B 13, 55 (2000).

[108] K. Okunishi, Y. Hieida and Y. Akutsu, Phys. Rev. B 60, R6953 (1999).

[109] N. Maeshima and K. Okunishi, Phys. Rev. B 62, 934 (2000).

[110] K. Okunishi and N. Maeshima, Phys. Rev. B 64, 212406 (2001).

[111] K. Okunishi and T. Tonegawa, J. Phys. Soc. Jpn. 72, 479 (2003).

[112] T. Tonegawa, K. Okamoto, K. Okunishi, K. Nomura, and M. Kaburagi, Physica B 346-347, 50 (2004).

[113] I. Affleck, Soliton Confinement and the Excitation Spectrum of Spin- Peierls Antiferromagnets, in Dynamical Properties of Unconventional Magnetic Systems, NATO ASI Series E, Vol. 349 (Kluwer Academic Publishers, 1998).

[114] E. Sorensen, I. Affleck, D. Augier, and D. Poilblanc, Phys. Rev. B 58, R14701 (1998).

[115] G. Bouzerar, A.P. Kampf, and G.I. Japaridze, Phys. Rev. B 58, 3117 (1998).

[116] A. Dobry and D. Ibaceta, Phys. Rev. B 59, 8660 (1999).

[117] C. Knetter and G.S. Uhrig, Eur. Phys. J. B 13, 209 (1999).

[118] T. Vekua, D.C. Cabra, A.O. Dobry, C.J. Gazza, and D. Poilblanc, Phys. Rev. Lett. 96, 117205 (2006); C.J. Gazza, A.O. Dobry, D.C. Cabra, and T. Vekua, Phys. Rev. B 75, $165104(2007)$.

[119] J.-B. Fouet, A. Lauchli, S. Pilgram, R.M. Noack, and F. Mila, Phys. Rev. B 73, 014409 (2006).

[120] P. Millet, J. Y. Henry, F. Mila, and J. Galy, J. Solid State Chem. 147, 676 (1999).

[121] J. L. Gavilano, D. Rau, S. Mushkolaj, H. R. Ott, P. Millet, and F. Mila, Phys. Rev. Lett. 90, 167202 (2003).

[122] J. L. Gavilano, E. Felder, D. Rau, H. R. Ott, P. Millet, F. Mila, T. Cichorek, and A. C. Mota, Phys Rev. B 72, 064431 (2005). 
[123] G. Seeber, P. Kögerler, B. M. Kariuki, and L. Cronin, Chem. Commun. (Cambridge) 2004, 1580 (2004).

[124] J. Schnack, H. Nojiri, P. Kögerler, G. T. Cooper and L. Cronin, Phys. Rev. B 70,174420 (2004).

[125] V. Subrahmanyam, Phys. Rev. B 52, 1133 (1995).

[126] F. Mila, Phys. Rev. Lett. 81, 2356 (1998).

[127] M. Hogh Jensen and P.S. Lomdahl, Phys. Rev. B 26, 1086 (1982).

[128] Z. Bajnok, L. Palla, G. Takacs, and F. Wagner, Nucl. Phys. B 601, 503 (2001).

[129] G. Delfino and G. Mussardo, Nucl. Phys. B 516, 675 (1998).

[130] H. H. Roomany and H. W. Wyld, Phys. Rev. D 21, 3341 (1980).

[131] K. Okamoto and K. Nomura, Phys. Lett. A 169, 433 (1992).

[132] K. Okamoto and K. Nomura, J. Phys. A 27, 5773 (1994).

[133] K. Nomura, J. Phys. A 28, 5451(1995).

[134] For a review, see e.g. J.-P. Boucher and L.-P. Regnault, J. Phys. I (France) 6, 1939 (1996).

[135] M. Isobe and Y. Ueda, J. Phys. Soc. Jpn. 65, 3142 (1996); N. Fujiwara, H. Yasuoka, M. Isobe, Y. Ueda, and S. Maegawa, Phys. Rev. B 55, R11945 (1997).

[136] M. Matsuda and K. Katsumata, J. Magn. Magn. Mater. 140, 1671 (1995).

[137] M. Hase, M. Kohno, H. Kitazawa, N. Tsujii, O. Suzuki, K. Ozawa, G. Kido, M. Imai, and X. Hu, Phys. Rev. B 73, 104419 (2006).

[138] F. Becca, F. Mila, and D. Poilblanc, Phys. Rev. Lett. 91, 067202 (2003).

[139] A.E. Feiguin, J.A. Riera, A. Dobry, and H.A. Ceccatto, Phys. Rev. B 56, 14607 (1997).

[140] P. W. Anderson, Mater. Res. Bull. 8, 153 (1973); P. Fazekas and P. W. Anderson, Philos. Mag. 30, 423 (1974).

[141] D. A. Huse and V. Elser, Phys. Rev. Lett. 60, 2531 (1988).

[142] Th. Jolicoeur and J. C. Le Guillou, Phys. Rev. B 40, 2727 (1989).

[143] B. Bernu, C. Lhuillier, and L. Pierre, Phys. Rev. Lett. 69, 2590 (1992); B. Bernu, P. Lecheminant, C. Lhuillier, and L.Pierre, Phys. Rev. B 50, 10048 (1994).

[144] L. Capriotti, A. E. Trumper, and S. Sorella, Phys. Rev. Lett. 82, 3899 (1999). 
[145] G. Misguich, C. Lhuillier, B. Bernu, and C. Waldtmann, Phys. Rev. B 60, 1064 (1999).

[146] S. Yunoki and S. Sorella, Phys. Rev. B 74, 014408 (2006), cond-mat/0602180.

[147] M. Q. Weng, D. N. Sheng, Z. Y. Weng, Robert J. Bursill, Phys. Rev. B 74, 012407 (2006), cond-mat/0508186.

[148] Weihong Zheng, John O. Fjaerestad, Rajiv R. P. Singh, Ross H. McKenzie, Radu Coldea, Phys. Rev. Lett. 96, 057201 (2006).

[149] X. Obradors et al., Solid State Commun. 65, 189 (1988); A. P. Ramirez, G. P. Espinosa and A. S. Cooper, Phys. Rev. Lett. 64, 2070 (1990); C. Broholm et al., ibid. 65, 3173 (1990).

[150] D. S. Greywall, Phys. Rev. B 41, 1842 (1990); V. Elser, Phys. Rev. Lett. 62, 2405 (1990).

[151] O. Janson, J. Richter and H. Rosner, Phys. Rev. Lett. 101, 106403 (2008).

[152] O. Janson, J. Richter and H. Rosner, J. Phys.: Conf. Ser. 145012008.

[153] L. O. Manuel, A. E. Trumper, C. J. Gazza and H. A. Ceccatto, Phys. Rev. B 50, 1313 (10984).

[154] S. Sachdev, Phys. Rev. B , 45, 12377 (1992).

[155] Yoshitami Ajiro, J. Phys Soc. Jpan. Vol. 72 (2003).

[156] S. Okubo et al, J. Phys.: Conf. Series 200, 022042 (2010).

[157] R. Arita et al., Phys. Rev. Lett. 88, 127202 (2002); Y. Suwa et al., Phys. Rev. B 68, 174419 (2003); A. Harrison, J. Phys.: Condens. Matter 16, S553 (2004); Y. Z. Zheng et al., Angew. Chem. Int. Ed. 46, 6076 (2007).

[158] Yoko Miura, Yukio Yasui, Taketo Moyoshi, Masatoshi Sato, Kazuhisa Kakurai, J. Phys. Soc. Jpn. 77 (2008) 104709.

[159] J.B. Fouet, P. Sindzingre, C. Lhuillier, Eur. Phys. J. B 20, 241 (2001).

[160] E. Rastelli, A. Tassi and L. Reatto, Physica 97B, 1 (1979).

[161] M. P. Gelfand, R. R. P. Singh and D. A. Huse, Phys. Rev. B 40, 10801 (1989).

[162] M. P. Gelfand. Phys. Rev. B 42, 8206 (1990).

[163] R. R. P. Singh, Zheng Weihong, C. J. Hamer, and J. Oitmaa, Phys. Rev. B 60, 7278 (1999).

[164] V. N. Kotov, J. Oitmaa, O. Sushkov and Zheng Weihong, Phil. Mag. B 80, 1483 (2000). 
[165] L. Capriotti, F. Becca, A. Parola, and S. Sorella, Phys. Rev. Lett. 87, 097201 (2001).

[166] O. P. Sushkov, J. Oitmaa, and Zheng Weihong, Phys. Rev. B 66, 054401 (2002).

[167] O. P. Sushkov, J. Oitmaa, and Zheng Weihong, Phys. Rev. B 63, 104420 (2001).

[168] H. J. Schulz, T. A. L. Ziman, Europhys. Lett. 18, 355 (1992).

[169] T. Einarsson and H. J. Schulz, Phys. Rev. B 51, 6151 (1995).

[170] H. J. Schulz, T. A. L. Ziman, D. Poilblanc, J. Physique I 6, 675 (1996).

[171] A. W. Sandvik, Phys. Rev. B 56, 11678 (1997).

[172] S. Sorella, Phys. Rev. Lett. 80, 4558 (1998).

[173] S. Sorella, Phys. Rev. B 64, 024512 (2001).

[174] I. Affleck, in Strings, Fields and Critical Phenomena, Les Houches Summer school, Session XLIX, edited by E. Brezin and J. Zin-Justin, North Holland, (1990).

[175] J. Cardy Scaling and renormalization in statistical physics Cambridge University Press (1996).

[176] H. Frahm and V. E. Korepin, Phys. Rev. B 42, 10553 (1990).

[177] H. Frahm and V. E. Korepin, Phys. Rev. B 43, 5653 (1991).

[178] J.H.P. Colpa, Physica 93A, 327-353 (1978). 



\section{Apéndice A}

\section{.1. Bosonización Abeliana, $d=1$}

En este apéndice damos más detalles técnicos sobre Bosonización Abeliana. Las convenciones usadas para los signos en la expresión de los campos fermionicos es muy variada en la literatura. Nosotros usamos las reglas usadas en las referencias [45, 174]. En la primera sección reescribimos el hamiltoniano de Hubbard en el límite continuo, en términos de campos fermiónicos. La segunda sección es dedicada a la bosonización del hamiltoniano de Hubbard. Finalmente, el tercera sección agregamos la interacción con la red, que requiere una bosonización con más armónicos $[86,176,177]$.

\section{.1.1. Teoría efectiva de baja energía del modelo de Hubbard}

Consideremos el hamiltoniano de Hubbard unidimensional

$$
\mathcal{H}_{t-U-\mu}=-t \sum_{\sigma_{\uparrow, \downarrow}} \sum_{j=1}^{N}\left(c_{\sigma, j}^{\dagger} \cdot c_{\sigma, j+1}+\text { h.c. }\right)+U \sum_{j=1}^{N} n_{\uparrow, j} n_{\downarrow, j}+\mu \sum_{j=1}^{N} n_{j}
$$

Para el caso $U=0$, correspondiente al hamiltoniano de fermiones libres, el estado fundamental se obtiene ocupando todos los estados de partícula independiente con energía menor que la energía de Fermi, o bien trabajando en el espacio de impulsos, corresponde a ocupar estados con impulsos en el intervalo $\left(-k_{F, \sigma}, k_{F, \sigma}\right)$ (ver sección 2.1.1). $k_{F, \sigma}$ (con $\sigma=\uparrow, \downarrow$ ) son los vectores de Fermi de cada sector de espín. A partir de estos, se pueden definir nuevos vectores de onda $k_{ \pm}$relacionados con la densidad del sistema y la magnetización

$$
\begin{aligned}
& k_{+}=k_{F, \uparrow}+k_{F, \downarrow} \equiv \pi n \\
& k_{-}=k_{F, \uparrow}-k_{F, \downarrow} \equiv \pi\langle M\rangle
\end{aligned}
$$

donde $n$ es la densidad de electrones $n=N_{e} / N$, y $\langle M\rangle$ la magnetización normalizada del sistema dada por $\langle M\rangle=\left(N_{\uparrow}-N_{\downarrow}\right) / N_{e}$. En el caso sin campo magnético externo, los vectores $k_{F, \sigma}$ son iguales quedando

$$
\begin{aligned}
k_{F, \uparrow} & \equiv k_{F, \downarrow} \equiv k_{F} \equiv n \frac{\pi}{2} \\
k_{-} & =0 \\
k_{+} & =n \pi
\end{aligned}
$$


Dado que las excitaciones de baja energía son construídas al considerar partículas con impulsos en la vecindad de los puntos de Fermi $\pm k_{F, \sigma}$, vamos a descomponer el campo fermiónico en las componentes izquierda $\left(k_{F, \sigma}\right)$ y derecha $\left(k_{F, \sigma}\right)$.

Como discutimos en la sección 2.1, los operadores de creación y aniquilación en términos de los campos fermiónicos izquierdos y derechos se expresan

$$
\begin{aligned}
c(x) & \equiv \frac{c_{j}}{\sqrt{a}} \\
& \equiv \psi_{R}(x) e^{i k_{F} x}+\psi_{L}(x) e^{-i k_{F} x} \ldots
\end{aligned}
$$

donde ... indican términos de orden superior en el desarrollo de baja energía. Los campos $\psi_{R}(x)$ y $\psi_{L}(x)$ se pueden reescribir términos de nuevos operadores de electrones y huecos $\alpha_{\sigma}(k)$ y $\beta_{\sigma}(k)(\sigma=\uparrow, \downarrow)$ alrededor de los puntos de Fermi,

$$
\begin{aligned}
\psi_{R, \sigma}(x, t) & =\int_{k>0} \frac{d k}{2 \pi}\left[e^{i k(t-x)} \alpha_{\sigma}(k)+e^{-i k(t+x)} \beta_{\sigma}^{\dagger}(k)\right] \\
\psi_{L, \sigma}(x, t) & =\int_{k<0} \frac{d k}{2 \pi}\left[e^{-i k(t+x)} \alpha_{\sigma}(k)+e^{-i k(t+x)} \beta_{\sigma}^{\dagger}(k)\right]
\end{aligned}
$$

Nosotros estamos interesados en el caso independiente del tiempo (o bien $t=0$ ), tal que los campos dependen únicamente de la variable $x=m a$, donde $m \in Z$ y $a$ es el espaciamiento de la red. La inclusión de la coordenada temporal querará clara cuando introduzcamos coordenadas complejas.

Ahora vamos a reescribir el producto de operadores de creación/aniquilación en término de los operadores de campo:

$$
\begin{aligned}
\frac{1}{a} c_{\sigma, j}^{\dagger} c_{\sigma, j}= & \psi_{\sigma}^{\dagger}(x) \psi_{\sigma}(x) \\
= & e^{i k_{F, \sigma} a} J_{L, \sigma}(x)+e^{-i k_{F, \sigma} a} J_{R, \sigma}(x) \\
& +a e^{i k_{F, \sigma} a} \psi_{L, \sigma}^{\dagger}(x) \partial_{x} \psi_{L, \sigma}(x)+a e^{-i k_{F, \sigma} a} \psi_{R, \sigma}^{\dagger}(x) \partial_{x} \psi_{R, \sigma}(x) \\
& +e^{-i k_{F, \sigma}(2 x+a)} \psi_{L, \sigma}^{\dagger}(x) \psi_{R, \sigma}(x)+e^{i k_{F, \sigma}(2 x+a)} \psi_{R, \sigma}^{\dagger}(x) \psi_{L, \sigma}(x) \\
& +a e^{i k_{F, \sigma}(2 x+a)} \psi_{R, \sigma}^{\dagger}(x) \partial_{x} \psi_{L, \sigma}+a e^{-i k_{F, \sigma}(2 x+a)} \psi_{L, \sigma}^{\dagger}(x) \partial_{x} \psi_{R, \sigma}
\end{aligned}
$$

donde los términos $J_{r, \sigma}(x)(r=R, L)$ son las densidades de los campos quirales:

$$
J_{r, \sigma}(x) \equiv \psi_{r, \sigma}^{\dagger} \psi_{r, \sigma}
$$

De manera análoga, en el límite continuo, el operador número que aparece en el potencial químico queda

$$
\begin{aligned}
\frac{1}{a} n_{r, \sigma} & =\psi_{r, \sigma}^{\dagger} \psi_{r, \sigma} \\
& =J_{R, \sigma}(x)+J_{L, \sigma}(x)+e^{-i 2 k_{F, \sigma} x} \psi_{L, \sigma}^{\dagger}(x) \psi_{R, \sigma}(x)+e^{i 2 k_{F, \sigma} x} \psi_{R, \sigma}^{\dagger}(x) \psi_{L, \sigma}(x)
\end{aligned}
$$


De las expresiones (6) y (8) se puede observar que hay términos no oscilatorios y otros oscilatorios cuya longitud de onda depende de $k_{F, \sigma}$. Si un dado hamiltoniano contiene estos términos sin singuna función dependiente de la posición, solo los factores no oscilatorios son relevantes en el sentido que no integran a cero [40]. Sin embargo, como veremos en la sección (.1.4) cuando incluyamos la interacción con deformaciones de la red (fonones en el límite adiabático), estos factores pueden conmensurar jugando un papel muy importante en la descripción de baja energía de sistema.

\section{Caso no interactuante $(U=0)$}

Primero nos vamos a enfocar en el gas de electrones libres en una red unidimensional de $N$ sitios, con una integral de hopping $t$ y potencial químico $\mu$ que fija el número de electrones $N_{e}$

$$
\mathcal{H}_{t-\mu}=-t \sum_{\sigma_{\uparrow, \downarrow}} \sum_{j=1}^{N}\left(c_{\sigma, j}^{\dagger} \cdot c_{\sigma, j+1}+\text { h.c. }\right)+\mu \sum_{j=1}^{N} n_{j}
$$

En el siguiente resultado, los términos escilatorios son directamente descartados por el argumento mencionado al final de la sección anterior. Utilizando las expresiones de los operadores fermiónicos en términos de los campos fermionicos (6) y (8) en le límite continuo, el hamiltoniano (9) queda

$$
\begin{aligned}
\mathcal{H}_{t-\mu}= & -t \sum_{\sigma} \int d x\left[e^{i k_{F, \sigma} a} J_{L, \sigma}(x)+e^{-i k_{F, \sigma} a} J_{R, \sigma}(x)\right. \\
& \left.a\left(e^{i k_{F, \sigma} a} \psi_{L, \sigma}^{\dagger}(x) \partial_{x} \psi_{L, \sigma}(x)+e^{-i k_{F, \sigma} a} \psi_{R, \sigma}^{\dagger}(x) \partial_{x} \psi_{R, \sigma}(x)\right)+\text { h.c. }\right] \\
& +\mu \sum_{\sigma} \int d x\left[J_{L, \sigma}(x)+J_{R, \sigma}(x)\right]
\end{aligned}
$$

El potencial químico a temperatura cero es igual a la energía de Fermi:

$$
\mu=2 t \cos \left(k_{F} a\right)
$$

de esta manera, se puede ver que al tener ambos términos (cinético y de potencial químico), las corrientes $J_{\text {r sigma }}$ se cancelan mutuamente quedando

$$
\mathcal{H}_{t-\mu}=i v_{F} \sum_{\sigma} \int d x\left[\psi_{R, \sigma}^{\dagger}(x) \partial_{x} \psi_{R, \sigma}(x)-\psi_{L, \sigma}^{\dagger}(x) \partial_{x} \psi_{L, \sigma}(x)\right]
$$

donde la velocidad de Fermi $v_{F}$ juega el rol de la velocidad de la luz y está dada por ${ }^{1}$.

$$
\left.\frac{\partial \epsilon(k)}{\partial_{k}}\right|_{k=k_{F}} \equiv 2 \operatorname{tasin}\left(k_{F} a\right) \equiv v_{F}
$$

\footnotetext{
${ }^{1}$ En caso de tener un término de acoplamiento con un campo magnético, la consecuencia seria que los sectores $\uparrow \downarrow$ tendrían velocidades $v_{F \uparrow}=v_{F \downarrow}$
} 
Caso interactuante $(U \neq 0)$

Consideremos ahora adicionalmente el término de interacción coulombiana,

$$
\mathcal{H}_{U}=U \sum_{j} n_{\uparrow j} n_{\downarrow j} .
$$

Usando (8) y removiendo los factores oscilatorios obtenemos

$$
\begin{aligned}
\mathcal{H}_{U} & =a U \int d x\left[\left(J_{R, \uparrow}+J_{L, \uparrow}\right)\left(J_{R, \downarrow}+J_{L, \downarrow}\right)\right. \\
& \left.+\left(e^{i 2 k_{+} x} \psi_{R, \uparrow}^{\dagger} \psi_{L, \uparrow} \psi_{R, \downarrow}^{\dagger} \psi_{L, \downarrow}+\text { h.c. }\right)+\left(e^{i 2 k_{-} x} \psi_{R, \uparrow}^{\dagger} \psi_{L, \uparrow} \psi_{L, \downarrow}^{\dagger} \psi_{R, \downarrow}+\text { h.c. }\right)\right] \\
& =a U \int d x\left[\left(J_{R, \uparrow}+J_{L, \uparrow}\right)\left(J_{R, \downarrow}+J_{L, \downarrow}\right)\right. \\
& \left.+\left(e^{i 4 k_{F} x} \psi_{R, \uparrow}^{\dagger} \psi_{L, \uparrow} \psi_{R, \downarrow}^{\dagger} \psi_{L, \downarrow}+\text { h.c. }\right)+\left(e^{i 4 k_{F} x} \psi_{R, \uparrow}^{\dagger} \psi_{L, \uparrow} \psi_{L, \downarrow}^{\dagger} \psi_{R, \downarrow}+\text { h.c. }\right)\right]
\end{aligned}
$$

donde hemos usado que $k_{+}=2 k_{F}$ y $k_{-}=0$.

Fuera de medio llenado (es decir, $n \neq 1$ ), los términos oscilatorios $\propto e^{i 4 k_{F} x}$ pueden ser descartados y el hamiltoniano queda

$$
\mathcal{H}_{U}=a U \int d x\left[\left(J_{R, \uparrow}+J_{L, \uparrow}\right)\left(J_{R, \downarrow}+J_{L, \downarrow}\right)+\left(\psi_{R, \uparrow}^{\dagger} \psi_{L, \uparrow} \psi_{L, \downarrow}^{\dagger} \psi_{R, \downarrow}+\text { h.c. }\right)\right]
$$

A medio llenado $(n=1)$, el término $\propto e^{i 4 k_{F} x}=1$ es conmensurado y corresponde a la dispersión de partículas desde un lado de la superficie de Fermi hacia el otro. Este proceso de dispersión se denomina umklapp:

$$
\begin{aligned}
\mathcal{H}_{\text {umklampp }} & =a U \int d x\left(e^{i 4 k_{F} x} \psi_{R, \uparrow}^{\dagger} \psi_{L, \uparrow} \psi_{R, \downarrow}^{\dagger} \psi_{L, \downarrow}+\text { h.c. }\right) \\
& =a U \int d x\left(\psi_{R, \uparrow}^{\dagger} \psi_{L, \uparrow} \psi_{R, \downarrow}^{\dagger} \psi_{L, \downarrow}+\text { h.c. }\right)
\end{aligned}
$$

\section{.1.2. Teorías conformes}

Antes de mostrar las reglas de bosonización, es necesario introducir algunos conceptos muy importantes como simetría conforme y dimensión conforme de operadores. En esta sección vamos a presentar brevemente algunos conceptos básicos y necesarios para poder desarrollar la técnica de bosonización abeliana. Para una explicación más completa y detallada el lector interesado puede consultar amplia bibliografía del tema, por ejemplo $J$. Cardy [175] o P. Di Francesco el al [29].

Un punto fijo de una teoría, definida por el conjunto de acoplamientos $g_{1}, \ldots, g_{n}$, corresponde a un punto en el espacio de parámetros, tal que la teoría es invariante frente al cambio de cualquier tipo de escala). Una teoría con invariancia frente a rotaciones, traslaciones y cambios de escala se dice que tiene invariancia conforme. Para el tratamiento más formal 
de las teorías conformes, es conveniente introducir coordenadas complejas $z$ y $\bar{z}$ definidas en términos de la coordenada espacial $x$ y el tiempo imaginario $\tau=i t$,

$$
z=-i(x-v t)=v \tau-i x \bar{z}=-i(x+v t)=v \tau+i x
$$

con las correspondientes derivadas

$$
\begin{aligned}
\partial_{z} & =-\frac{i}{2}\left(\frac{1}{v} \partial_{t}-\partial_{x}\right) & \partial_{x} & =-i\left(\partial_{z}-\partial_{\bar{z}}\right) \\
\partial_{\bar{z}} & =-\frac{i}{2}\left(\frac{1}{v} \partial_{t}+\partial_{x}\right) & \partial_{t} & =-i v\left(\partial_{z}+\partial_{\bar{z}}\right)
\end{aligned}
$$

Un operador $O(z, \bar{z})$ perteneciente a una teoría de campos conforme es llamado primario si este escalea bien bajo una transformación conforme, es decir

$$
O^{\prime}(\alpha z, \bar{\alpha} \bar{z})=\alpha^{-h} \bar{\alpha}^{-\bar{h}} O(z, \bar{z}) .
$$

Las constantes $h$ y $\bar{h}$ son una propiedad fundamental del operador $O$ y son llamadas dimensiones conformes izquierdas y derechas respectivamente. Bajo una dilatacion pura $(\bar{\alpha}=\alpha)$, el operador escalea como $O^{\prime}(\alpha z, \alpha \bar{z})=\alpha^{-\Delta} O(z, \bar{z})$, donde $\Delta=\alpha+\bar{\alpha}$ es la dimensión de escala ordinaria de un operador. Bajo una rotación, tal que $\alpha=e^{i \theta}$ y $\bar{\alpha}=e^{-i \theta}$, el operador transforma como $O^{\prime}\left(e^{i \theta} z, e^{-i \theta}, \bar{z}\right)=e^{i s \theta} O(z, \bar{z})$, donde $s=h-\bar{h}$ es llamada espín conforme del operador.

La dimensión de escala de un operador aparece directamente en la función de correlación del operador, que es fijada por argumentos de escala, salvo una constante multiplicativa, como:

$$
\left\langle O(z, \bar{z}) O^{\dagger}(0,0)\right\rangle=\frac{1}{z^{2 h}} \frac{1}{\bar{z}^{2 \bar{h}}}
$$

\section{Efecto de las perturbaciones}

Consideremos la acción perturbada

$$
S=S_{o}+g \int d t d x O(x, t)
$$

donde $S_{o}$ es la acción de una teoría en un pnto fijo y $O$ denota un operador con dimensión de escala $\Delta$. Se puede mostrar que bajo un paso infinitesimal del grupo de renormalización, con un factor de escala $\lambda=1+d \Lambda / \Lambda$ actuando sobre los vectores de onda; la inversa del factor de escala actúa sobre las coordenadas, y si $x$ y $t$ representan las nuevas coordenadas reescaleadas; suficientemente cerca del punto fijo tenemos

$$
\begin{aligned}
S^{\prime} & =S_{o}+g \int d(\lambda t) d(\lambda x) O(\lambda x, \lambda t) \\
& =S_{o}+g \lambda^{2-\Delta} \int d t d x O(x, t)
\end{aligned}
$$


Integrando tal tranformación infinitesimal de escala, se puede mostrar que la constante de acoplamiento escalea de la forma

$$
\frac{g}{g_{o}}=\left(\frac{\xi_{o}}{\xi}\right)^{2-\Delta}
$$

donde $\xi$ y $\xi_{o}$ son las longitudes de correlación. Luego, la perturbación es relevante si $\Delta<2$, irrelevante si $\Delta>2$ y marginal si $\Delta=2$.

\section{.1.3. Reglas de bosonización}

Las componentes izquierda y derecha de los campos fermiónicos, $\psi_{L, \sigma}$ y $\psi_{R, \sigma}$ introducidos en la ecuación (4), están definidos en términos de los respectivos campos bosónicos $\phi_{L, \sigma} \mathrm{y}$ $\phi_{R, \sigma}$ como

$$
\begin{aligned}
& \psi_{R, \sigma}(z)=\frac{1}{\sqrt{2 \pi a}} \eta_{R, \sigma}: e^{i \sqrt{4 \pi} \phi_{R, \sigma}(z)}: \\
& \psi_{L, \sigma}(\bar{z})=\frac{1}{\sqrt{2 \pi a}} \eta_{L, \sigma}: e^{-i \sqrt{4 \pi} \phi_{L, \sigma}(\bar{z})}:
\end{aligned}
$$

donde los operadores $e^{i \sqrt{4 \pi} \phi_{r, \sigma}}$ son llamados operadores de vértice [29]. La notación : $e^{i \sqrt{4 \pi} \phi_{r, \sigma}}$ : indica órden normal de operadores. Esta consiste en poner todos los operadores de aniquilación a la derecha de los operadores de creación en la expansión de los operadores de vértice. Las cantidades $\eta_{r, \sigma}(r=L, R)$ son los factores de Klein que garantizan las relaciones de anti-conmuntación de los campos fermiónicos bosonizados.

A partir de los campos bosónicos quirales izquierdos y derechos, se introducen los campos bosónicos $\phi_{\sigma}$ y su dual $\theta_{\sigma}$,

$$
\begin{aligned}
\varphi_{\sigma}(x, t) \equiv \varphi_{\sigma}(z, \bar{z}) & =\phi_{R, \sigma}(z)+\phi_{L, \sigma}(\bar{z}) \\
\theta_{\sigma}(x, t) \equiv \theta_{\sigma}(z, \bar{z}) & =\phi_{R, \sigma}(z)-\phi_{L, \sigma}(\bar{z})
\end{aligned}
$$

mientras que las derivadas con respecto a $z$ y $\bar{z}$ son

$$
\begin{aligned}
\partial_{z} \varphi_{\sigma}(z, \bar{z}) & =\partial_{z} \phi_{R, \sigma}(z) \\
\partial_{\bar{z}} \varphi_{\sigma}(z, \bar{z}) & =\partial_{\bar{z}} \phi_{L, \sigma}(\bar{z})
\end{aligned}
$$

Las funciones de correlación de los campos $\psi_{R, \sigma}(z)$ y $\psi_{L, \sigma}(\bar{z})$ están dada por [29]

$$
\begin{aligned}
\left\langle\phi_{R, \sigma}(z) \phi_{R, \sigma}(w)\right\rangle & =-\frac{1}{4 \pi} \log (z-w) \\
\left\langle\phi_{L, \sigma}(z) \phi_{L, \sigma}(w)\right\rangle & =-\frac{1}{4 \pi} \log (\bar{z}-\bar{w}) \\
\left\langle\phi_{R, \sigma}(z) \phi_{L, \sigma}(\bar{w})\right\rangle & =0
\end{aligned}
$$

Los productos de fermiones vienen, después de bosonizar, en producto de operadores de vértices. Si los campos bosónicos que intervienen en los exponentes poseen diferentes grados 
de libertad, es decir distinto índice de espín $\sigma \neq \sigma^{\prime}$ o de sector $R, L$, estos productos son simples productos de exponenciales. En caso contrario, debido al orden normal de operadores, uno tiene que tener en cuenta las correlaciones entre los campos bosónicos

$$
\begin{aligned}
: e^{i \alpha \phi_{\sigma, R}(z)}:: e^{i \beta \phi_{\sigma, R}(w)}: & =: e^{i\left(\alpha \phi_{\sigma, R}(z)+\beta \phi_{\sigma, R}(w)\right)}: e^{-\alpha \beta\left\langle\phi_{\sigma, R}(z) \phi_{\sigma, R}(w)\right\rangle} \\
& =: e^{i\left(\alpha \phi_{\sigma, R}(z)+\beta \phi_{\sigma, R}(w)\right)}:|z-w|^{\frac{\alpha \beta}{4 \pi}} \\
: e^{i \alpha \phi_{\sigma, L}(\bar{z})}:: e^{i \beta \phi_{\sigma, L}(\bar{w})}: & =e^{i\left(\alpha \phi_{\sigma, L}(\bar{z})+\beta \phi_{\sigma, L}(\bar{w})\right)}:|\bar{z}-\bar{w}|^{\frac{\alpha \beta}{4 \pi}}
\end{aligned}
$$

Con estas identidades y la ecuación (20) podemos calcular las dimensiones conformes de los operadores de vértice

$$
\begin{aligned}
\left\langle: e^{i \alpha \phi_{\sigma, R}(z)}:\left(: e^{-i \alpha \phi_{\sigma, R}(w)}:\right)^{\dagger}\right\rangle & =e^{-\frac{\alpha^{2}}{4 \pi} \log |z-w|} \\
& =\frac{1}{|z-w|^{\frac{\alpha^{2}}{4 \pi}}} \\
\left\langle: e^{i \alpha \phi_{\sigma, L}(\bar{z})}:\left(: e^{-i \alpha \phi_{\sigma, L}(\bar{w})}:\right)^{\dagger}\right\rangle & =\frac{1}{|\bar{z}-\bar{w}|^{\frac{\alpha^{2}}{4 \pi}}}
\end{aligned}
$$

de donde se extrae que la dimensiones de los operadores de vértices

$$
\begin{aligned}
\operatorname{dim}\left[e^{i \alpha \phi_{\sigma, a}(z)}\right] & =\frac{\alpha^{2}}{8 \pi} \\
\operatorname{dim}\left[e^{i \alpha \varphi_{\sigma}(z, \bar{z})}\right] & =\frac{\alpha^{2}}{4 \pi}
\end{aligned}
$$

\section{Bosonización con más armónicos.}

En la sección 2.1.1 presentamos una expresión del operador fermionico $\psi_{\sigma}(x)$ (ecuación (2.15)) alrededor del nivel de Fermi en término de operadores $\psi_{L, \sigma}(x)$ y $\psi_{R, \sigma}(x)$. En un trabajo reciente D. Cabra et. al [86] presentaron un desarollo más general construido a partir de las expresiones de las funciones de correlación del modelo de Hubbard [176, 177]. Este desarrollo está dado porque

$$
\begin{aligned}
\psi_{\downarrow}(x)= & e^{-i k_{F, \downarrow} x} \psi_{R, \downarrow}(x)\left[r_{1}+r_{2} e^{-i 2 k_{F, \uparrow} x} \psi_{L, \uparrow}^{\dagger}(x) \psi_{R, \uparrow}(x)+r_{3} e^{2 i k_{F, \uparrow} x} \psi_{R, \uparrow}^{\dagger}(x) \psi_{L, \uparrow}(x)+\ldots\right] \\
& +e^{i k_{F, \downarrow} x} \psi_{L, \downarrow}(x)\left[l_{1}+l_{2} e^{2 i k_{F, \uparrow} x} \psi_{R, \uparrow}^{\dagger}(x) \psi_{L, \uparrow}(x)+l_{3} e^{-2 i k_{F, \uparrow} x} \psi_{L, \uparrow}^{\dagger}(x) \psi_{R, \uparrow}(x)+\ldots\right] \\
\psi_{\uparrow}(x)= & \downarrow \rightleftarrows \uparrow
\end{aligned}
$$

donde

$$
\begin{aligned}
\psi_{R, \sigma} & =\frac{\eta_{R, \sigma}}{\sqrt{2 \pi}} e^{i \sqrt{\pi} \phi_{R, \sigma}(x)} \\
\psi_{L, \sigma} & =\frac{\eta_{L, \sigma}}{\sqrt{2 \pi}} e^{-i \sqrt{\pi} \phi_{L, \sigma}(x)}
\end{aligned}
$$

En (39) $, r_{i}, l_{i}$ son constantes numéricas no-universales. Mediante cálculos perturbativos [86] se conoce que para el hamiltoniano de Hubbard, $r_{1}, l_{1} \sim 1+\alpha U$ y $r_{2}, r_{3}, l_{2}, l_{3} \sim U$. 
Utilizando las ecuaciones (39) y (40) se puede mostrar que la expresión bosonizada del operador densidad (sin campo magnético externo) es,

$$
\begin{aligned}
n(x)= & \psi_{\uparrow}^{\dagger}(x) \psi_{\uparrow}(x)+\psi_{\downarrow}^{\dagger}(x) \psi_{\downarrow}(x) \\
= & \frac{1}{2}+\frac{1}{\sqrt{\pi}} \frac{d\left(\varphi_{\uparrow}(x)+\varphi_{\downarrow}(x)\right)}{d x} \\
& -4 r_{1} l_{1} \sin \left[2 k_{F} x+\sqrt{\pi}\left(\varphi_{\uparrow}(x)+\varphi_{\downarrow}(x)\right)\right] \cos \left[\sqrt{\pi}\left(\varphi_{\uparrow}(x)-\varphi_{\downarrow}(x)\right)\right] \\
& -4\left(r_{1} l_{2}+r_{2} l_{1}\right) \cos \left[4 k_{F} x+\sqrt{4 \pi}\left(\varphi_{\uparrow}(x)+\varphi_{\downarrow}(x)\right)\right]
\end{aligned}
$$

\section{.1.4. Bosonización del acoplamiento electrón-fonón, espín-fonón.}

En esta parte utilizaremos las reglas de bosonización para tratar el término de interacción de los fonones en el límite adiabático (sección 1.4), con los electrones y espines (ecuaciones (1.73) y (1.72) respectivamente). Para comenzar vamos a tratar el término de acoplamiento espín-fonón que es más sencillo y finalmente vamos tratar el acoplamiento electrón-fonón.

\section{Acoplamiento espín-fonón.}

Consideremos una cadena unidimensional, y para simplificar vamos a tomar la interacción solo a primeros vecinos. En ese caso, según la ecuación (1.72) tenemos

$$
\begin{aligned}
H_{\text {espín-fonón }} & \sim \alpha \sum_{j=1}^{N}\left(u_{j+1}-u_{j}\right) \mathbf{S}_{j} \cdot \mathbf{S}_{j+1} \\
& \sim \alpha \sum_{j=1}^{N} \delta_{j} \mathbf{S}_{j} \cdot \mathbf{S}_{j+1} .
\end{aligned}
$$

Tomando el límite continuo y utilizando la ecuación (2.57) obtenemos

$$
H_{\text {espín-fonón }} \sim \alpha \int d x \delta(x) \rho(x)
$$

donde $\rho(x)$ está dado por (2.57).

La contribución más importante en el límite de baja energía viene de la interferencia constructiva entre la modulación $\delta(x)$ y la densidad de energía $\rho(x)$.

Supongamos un patrón periódico con período $L_{p}$ a magnetización $M$, tal que $\delta\left(x+L_{p}\right)=\delta(x)$. Si $L_{p}$ es par, el ansatz más general para el término de modulación está dado porque

$$
\delta(x)=\delta_{\frac{L_{p}}{2}} \cos (\pi x)+\sum_{n=1}^{\frac{L_{p}}{2}-1} \delta_{n} \cos \left(n \frac{2 \pi x}{L_{p}}+\theta_{n}\right)
$$




\begin{tabular}{|c|c|c|c|}
\hline & $M=0$ & $M=1 / 3$ & $M=1 / 2$ \\
\hline$L_{p}$ & 4 & 6 & 8 \\
\hline$k_{F}$ & $\pi / 2$ & $\pi / 3$ & $\pi / 4$ \\
\hline$N_{w}$ & 2 & 3 & 4 \\
\hline $\begin{array}{c}\text { frecuencias } \\
\text { posibles }\end{array}$ & $2 k_{F}$ & $2 k_{F}$ & $2 k_{F} \mathrm{y} 4 k_{F}$ \\
\hline
\end{tabular}

Cuadro 1: Frecuancias posibles para las deformaciones de la red $\delta(x)$ para magnetizaciones $M=0,1 / 3$ y $1 / 2$.

donde $\delta_{\frac{L_{p}}{2}}, \delta_{n}$ son las amplitudes y $\theta_{n}$ las fases de los diferentes términos de la expansión. Si $L_{p}$ es impar, el primer término es cero y la suma va de $n=1$ a $n=\frac{\left(L_{p}-1\right)}{2}$.

A partir de las ecuaciones (2.57) y (44), vemos que el producto de los dos términos es conmensurado únicamente si las frecuencias características son proporcionales, es decir

$$
\begin{aligned}
n \frac{2 \pi}{L_{p}} & =k_{F} \\
& =\frac{\pi}{2}(1-M) \\
\Rightarrow L_{p} & =\frac{4 n}{1-M}
\end{aligned}
$$

con $n \in$ entero y $M \neq 1$. Consecuentemente, el número de frecuencias $N_{w}$ en la expansión de $\delta(x)$ es

$$
N_{w}=\left\{\begin{array}{l}
{\left[\frac{L_{p}}{2}\right], \text { si } L_{p} \text { es par }} \\
{\left[\frac{\left(L_{p}-1\right)}{2}\right], \text { si } L_{p} \text { es impar }}
\end{array}\right.
$$

Usando el desarrollo de $\delta(x)$, el término de interacción toma la forma

$$
H_{\text {espín-fonón }}=\sum_{n=0}^{N_{w}+4} \lambda_{n} \int d x \cos \left(n k_{F} x+\sqrt{2 \pi} \varphi(x)+\Gamma_{n}\right)
$$

donde $\Gamma_{n}$ es una función de $\theta_{n}, \mathrm{y} \lambda_{n}$ es una función de $\delta_{n}$ y $\theta_{n}$.

De la expresión anterior, solo van a contribuir aquellos términos no oscilatorios, es decir $n k_{F}=2 \pi z$ (con $z$ entero).

En la tabla El ansatz para $\delta(x)$ que surge al pedir conmensurabilidad en la expresión bosonizada de la interacción espín-fonón, ha sido ampliamente verificado numéricamente mediante cálculos numéricos autoconsistentes siguiendo el procedimiento debido Feiguin, et al [139].

\section{Acoplamiento electrón-fonón.}

Para el caso de la interacción electón-fonón, el procedimiento es muy similar que se describió en la sección anterior. Para un patrón de deformaciones $\delta(x)$ de período $L_{p}$, la 
expresión más general está dada por la ecuación (44). La diferencia que surge, es que en el caso de electrones $k_{F}=n \frac{\pi}{2}$ donde $n$ es la densidad del sistema.

En el caso particular de llenado $1 / 4(n=1 / 2)$, que es el que trataremos con más detalle en el capítulo 3. Vamos a considerar dos clases de acoplamiento electrón-fonón: acoplamiento de Peierls, que modula los acoplamientos de hopping $t_{i j}$, y acoplamiento de Hosltein que modifica la energía en el sitio.

Utilizando la expresión (39), los factores oscilatorios de los términos de hopping y de densidad en el sitio quedan

$$
\begin{aligned}
\left(c_{\sigma}^{\dagger}(x) c_{\sigma}(x+a)+\text { h.c. }\right)= & -\alpha_{1} \sin \left(\frac{\pi}{2} x+\frac{\pi}{4}+\sqrt{2 \pi} \varphi_{c}\right) \cos \left(\sqrt{2 \pi} \varphi_{s}\right) \\
& -\alpha_{2} \sin \left(\pi x+2 \sqrt{2 \pi} \varphi_{c}\right) \\
c_{\sigma}^{\dagger}(x) c_{\sigma}(x)= & -\alpha_{1}^{\prime} \sin \left(\frac{\pi}{2} x+\sqrt{2 \pi} \varphi_{c}\right) \cos \left(\sqrt{2 \pi} \varphi_{s}\right) \\
& -\alpha_{2}^{\prime} \cos \left(\pi x+2 \sqrt{2 \pi} \varphi_{c}\right)
\end{aligned}
$$

La deformación más general a llenado 1/4 exhibe dos armónicos:

$$
\delta(x)=\delta_{2} \cos \left(2 k_{F} x+\beta_{2}\right)+\delta_{4} \cos \left(4 k_{F} x+\beta_{4}\right)
$$

donde $\beta_{2}$ y $\beta_{4}$ son constantes que determinan el patrón de la deformación, mientras que $\delta_{2}$ y $\delta_{2}$ miden la amplitud de cada modo. Finalmente, la expresión bosonizada de los acoplamientos de Peierls y Holstein son:

$$
\begin{aligned}
\left(c_{i, \sigma}^{\dagger} c_{i+1, \sigma}+\text { h.c. }\right)= & -\frac{\delta_{2} \alpha_{1}}{2} \sin \left(\sqrt{2 \pi} \varphi_{c}+\beta_{2}+\frac{\pi}{4}\right) \cos \left(\sqrt{2 \pi} \varphi_{s}\right) \\
& -\delta_{4} \alpha_{2} \sin \left(2 \sqrt{2 \pi} \varphi_{c}\right) \\
\left(c_{i, \sigma}^{\dagger} c_{i, \sigma}+\text { h.c. }\right)= & \delta_{2} \alpha_{1}^{\prime} \sin \left(\beta_{2}-\sqrt{2 \pi} \varphi_{c}\right) \cos \left(\sqrt{2 \pi} \varphi_{s}\right) \\
& -2 \delta_{4} \alpha_{2}^{\prime} \cos \left(2 \sqrt{2 \pi} \varphi_{c}\right)
\end{aligned}
$$




\section{Apéndice B}

\section{.2. Diagonalización paraunitaria.}

Muy pocos problemas de muchos cuerpos admiten una solución exacta. Entre ellos, uno de las formas más comunes son hamiltonianos cuadráticos que consisten de operadores de creación y aniquilación. En este apéndice mostramos brevemente el caso de hamiltonianos bosónicos siendo el caso de fermiones muy similar en cuanto al procedimiento.

La diagonalización de un hamiltoniano bosónico cuadrático de la forma

$$
\begin{aligned}
\mathcal{H} & =\sum_{\mathbf{k}} \Psi_{\mathbf{k}}^{\dagger} \cdot M(\mathbf{k}) \cdot \Psi_{\mathbf{k}} \\
\Psi_{\mathbf{k}} & =\left(a_{\mathbf{k}}^{(1)}, a_{\mathbf{k}}^{(2)}, \ldots, a_{\mathbf{k}}^{(N)}, a_{-\mathbf{k}}^{(1) \dagger}, a_{-\mathbf{k}}^{(2) \dagger}, \ldots, a_{-\mathbf{k}}^{(N) \dagger}\right)
\end{aligned}
$$

con $N$ el número de especies bonónicas y $M(\mathbf{k})$ una matriz hermítica requiere más que una simple transformación unitaria, debido a la necesidad de preservar las relaciones de conmutación bosónicas de los nuevos operadores. Se necesita entonces una transformación de Bogoliubov generalizada (diagonalización paraunitaria), cuyos detalles pueden ser consultados en [178]. Para diagonalizar el hamiltoniano (54), definimos una transformación

$$
\begin{aligned}
& \Upsilon_{\mathbf{k}}=T \cdot \Psi_{\mathbf{k}} \\
& \Upsilon_{\mathbf{k}}=\left(b_{\mathbf{k}}^{(1)}, b_{\mathbf{k}}^{(2)}, \ldots, b_{\mathbf{k}}^{(N)}, b_{-\mathbf{k}}^{(1) \dagger}, b_{-\mathbf{k}}^{(2) \dagger}, \ldots, b_{-\mathbf{k}}^{(N) \dagger}\right) .
\end{aligned}
$$

Tanto los operadores $a^{\prime} s$ y $b^{\prime} s$ deben satisfacer las relaciones de conmutación bosónicas,

$$
\begin{aligned}
& {\left[\Psi_{\mathbf{k}, \alpha}, \Psi_{\mathbf{k}^{\prime}, \beta}^{\dagger}\right]=\delta_{\mathbf{k}, \mathbf{k}^{\prime}}\left(\sigma^{3}\right)_{\alpha, \beta}} \\
& {\left[\Upsilon_{\mathbf{k}, \alpha}, \Upsilon_{\mathbf{k}^{\prime}, \beta}^{\dagger}\right]=\delta_{\mathbf{k}, \mathbf{k}^{\prime}}\left(\sigma^{3}\right)_{\alpha, \beta}}
\end{aligned}
$$

donde

$$
\sigma^{3}=\left(\begin{array}{cc}
I_{N \times N} & 0 \\
0 & -I_{N \times N}
\end{array}\right)
$$

usando las ecuaciones (55) y (58) tenemos

$$
\begin{aligned}
{\left[\Psi_{\mathbf{k}, \alpha}, \Psi_{\mathbf{k}^{\prime}, \beta}^{\dagger}\right] } & =T_{\alpha, \gamma}\left(T^{\dagger}\right)_{\delta, \beta}\left[\Upsilon_{\mathbf{k}, \gamma}, \Upsilon_{\mathbf{k}^{\prime}, \delta}^{\dagger}\right] \\
& \Downarrow \\
\sigma^{3} & =T \cdot \sigma^{3} \cdot T^{\dagger} .
\end{aligned}
$$


Utilizando esta transformación en el hamiltoniano (54) tenemos

$$
\mathcal{H}=\sum_{\mathbf{k}} \Upsilon_{\mathbf{k}}^{\dagger} \sigma_{3} T \sigma_{3} M(\mathbf{k}) T^{-1} \Upsilon_{\mathbf{k}}
$$

donde $\sigma_{3} T \sigma_{3} M(\mathbf{k}) T^{-1}=\tilde{M}(\mathbf{k})$ es una matriz diagonal.

Finalmente el hamiltoniano diagonal queda

$$
\begin{aligned}
\mathcal{H} & =\sum_{\mathbf{k}} \Upsilon_{\mathbf{k}}^{\dagger} \cdot \tilde{M}(\mathbf{k}) \cdot \Upsilon_{\mathbf{k}} \\
\mathcal{H} & =\sum_{\mathbf{k}, \alpha} 2 w(\mathbf{k})_{\alpha}\left(b_{\mathbf{k}}^{(\alpha) \dagger} \cdot b_{\mathbf{k}}^{(\alpha)}+\frac{1}{2}\right)
\end{aligned}
$$

donde $\alpha=1, \ldots, N$ y $w_{\alpha}(\mathbf{k})$ son los autovalores de la matriz $\tilde{M}(\mathbf{k})$. 
Esta tesis, realizada gracias a una beca de doctorado otorgada por CONICET, está basada en los siguientes trabajos científicos

* Quantum phase transitions in trimerized zigzag spin ladders. H. D. Rosales, D. C. Cabra, M. D. Grynberg, G. L. Rossini, y T. Vekua Phys. Rev. B 75, 174446 (2007).

* Spin-Peierls-like phases in magnetoelastic J1-J2 antiferromagnetic chains at 13 magnetization

H. D. Rosales y G. L. Rossini

Phys. Rev. B 76, 224404 (2007)

* Coexisting orders in the quarter-filled Hubbard chain with elastic deformations

H.D. Rosales, D.C. Cabra aceptado para publicación en European Physical Journal B

* Quantum disordered phase on the frustrated honeycomb lattice D.C. Cabra, C.A. Lamas and H.D. Rosales enviado para publicación a European Physical Letters. 